Functional characterization of the mevalonateisoprenoid biosynthesis pathway genes in Mucor circinelloides

\author{
Ph.D. dissertation
}

\title{
Dileep Kumar
}

Supervisors:

Prof. Dr. Csaba Vágvölgyi

Dr. Árpád Csernetics

Ph.D. School of Biology

Department of Microbiology

Faculty of Science and Informatics

University of Szeged

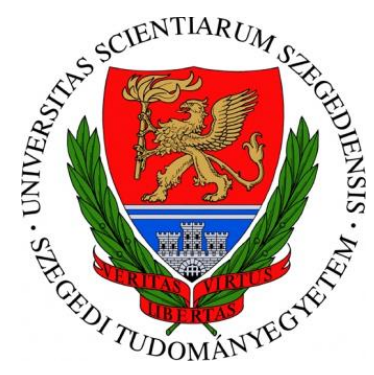

2018

Szeged 


\section{TABLE OF CONTENTS}

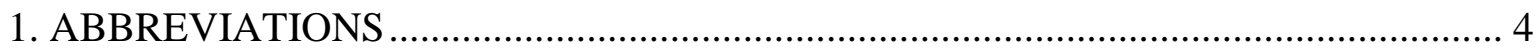

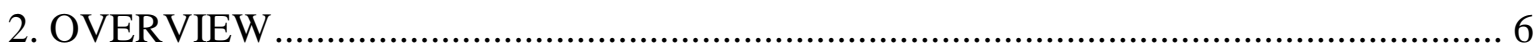

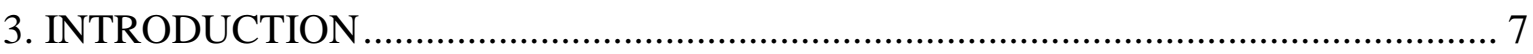

3.1. Characterization and taxonomy of Mucoromycotina fungi.............................................

3.2. Economic and clinical importance of Mucoromycotina fungi ....................................... 9

3.3. Antifungal agents applied in clinics to treat fungal infections .................................... 11

3.4. The mevalonate-isoprenoid biosynthesis pathway in $M$. circinelloides ...................... 15

3.5. Genetic background of the mevalonate-isoprenoid pathway in $M$.

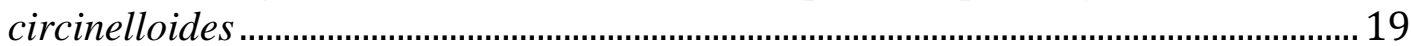

3.6. Genetic modification of Mucormycotina fungi............................................................. 21

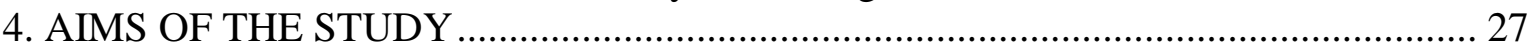

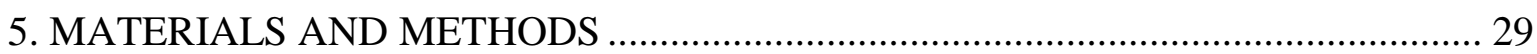

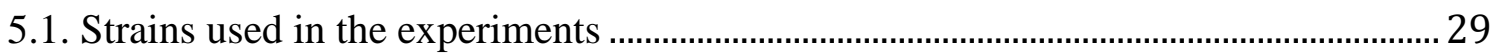

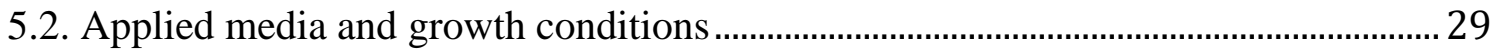

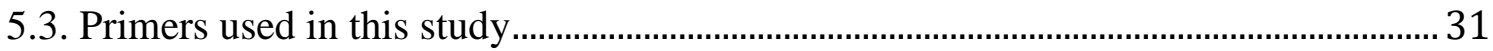

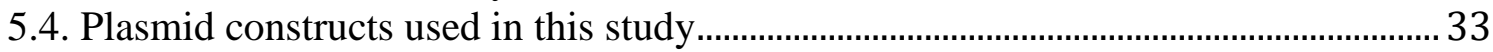

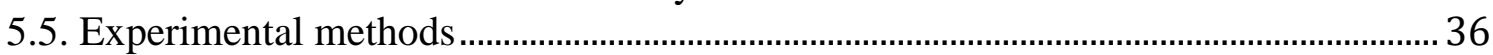

5.5.1. Extraction of genomic DNA from M. circinelloides.............................................. 36

5.5.2. Agarose gel electrophoresis and isolation of DNA from agarose gel .................. 37

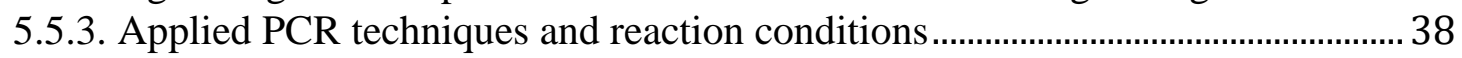

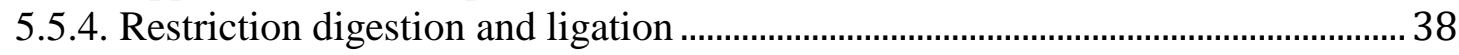

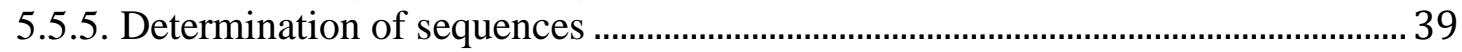

5.5.6. Preparation and transformation of competent E. coli cells and plasmid DNA

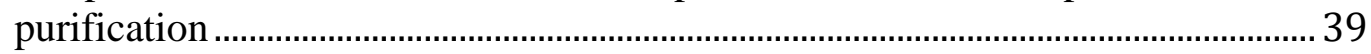

5.5.7. Total RNA purification from $M$. circinelloides and cDNA synthesis.................. 40

5.5.8. Quantitative real-time PCR ................................................................................... 40

5.5.9. Protoplast formation and $\mathrm{PEG} / \mathrm{CaCl}_{2}$-mediated transformation of $M$.

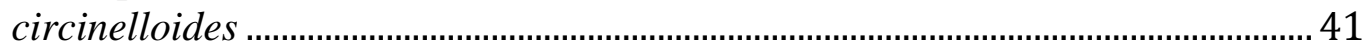

5.5.10. Microscopy and examination of mitotic stability of the mutants....................... 41

5.5.11. Determination of the carotenoid and ergosterol content in M. circinelloides 42

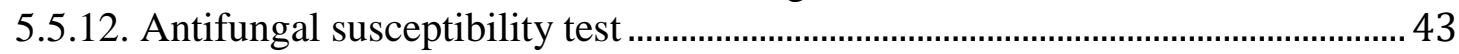

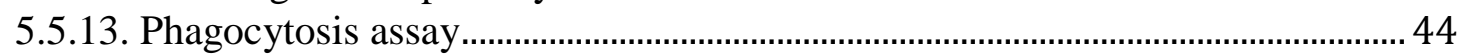

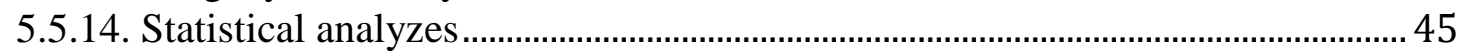

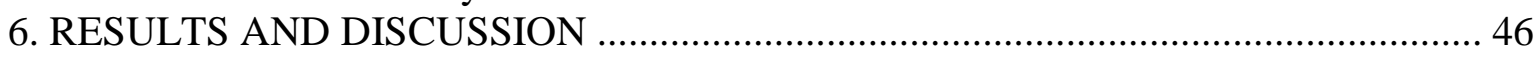

6.1. Transcription of six genes under different cultivation conditions involved

in the mevalonate-isoprenoid biosynthesis in M. circinelloides ....................................46

6.1.1. Comparison of relative transcription level of selected genes ................................46

6.1.2. Effect of environmental conditions on the transcription of selected $M$. circinelloides mevalonate-isoprenoid pathway genes

6.1.3. Effect of medium composition on the transcription of the $M$. circinelloides six mevalonate-isoprenoid pathway genes................................................................. 55

6.1.4 Effect of statin treatment on the transcription of the $M$. circinelloides six mevalonate-isoprenoid pathway genes

6.2. Construction of plasmids for overexpression and silencing of $M$. circinelloides six genes involved in the mevalonate-isoprenoid biosynthesis and transformation experiments. 
6.3. Characterization of the mevalonate-isoprenoid biosynthesis pathway mutant $M$. circinelloides strains.

6.3.1 Examination of plasmid copy number and relative transcription level change in the mutants

6.3.2 Investigation of macro- and micromorphology of the transformants

6.4. Carotenoid and ergosterol content of the M. circinelloides strains mutant in mevalonate-isoprenoid pathway

6.5. Antibiotic susceptibility of the $M$. circinelloides strains mutant in mevalonate-isoprenoid pathway

6.6. Interaction of the mevalonate-isoprenoid pathway mutant $M$. circinelloides strains with MH-S macrophages

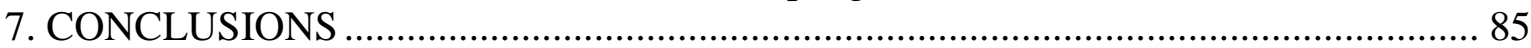

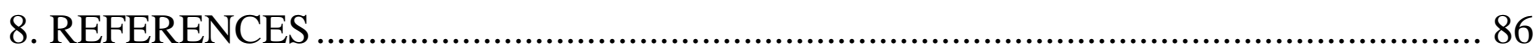

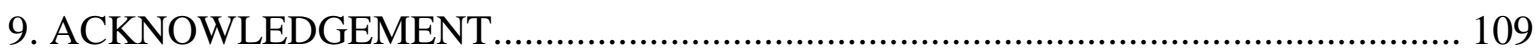

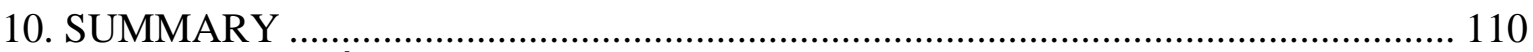

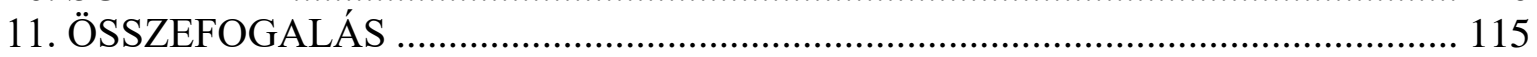

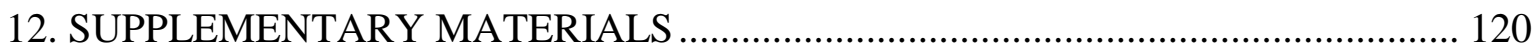




\section{ABBREVIATIONS}

\begin{tabular}{|c|c|}
\hline $\mathrm{AmB}$ & amphotericin B \\
\hline ATMT & Agrobacterium tumefaciens-mediated transformation \\
\hline \multirow[t]{2}{*}{ CBS } & Centraalbureau voor Schimmelcultures Fungal \\
\hline & Biodiversity Centre (CBS), Utrecht, The Netherlands \\
\hline cDNA & complementary DNA \\
\hline $\mathrm{CFU}$ & colony forming unit \\
\hline CLO & clotrimazole \\
\hline DHA & dihydroxyacetone \\
\hline DMAPP & dimethylallyl pyrophosphate \\
\hline DMSO & dimethyl sulfoxide \\
\hline EDTA & ethylenediamine tetraacetic acid \\
\hline EMBL & European Molecular Biology Laboratory \\
\hline FLU & fluvastatin \\
\hline FPP & farnesyl pyrophosphate \\
\hline \multirow[t]{2}{*}{ GHMP } & galacto-, homoserine-, mevalonate- and \\
\hline & phosphomevalonate kinases \\
\hline GGPP & geranylgeranyl pyrophosphate \\
\hline GPP & geranyl pyrophosphate \\
\hline HMG-CoA & 3-hydroxy-3-methylglutaryl coenzyme A \\
\hline HPLC & high performance liquid chromatography \\
\hline hpRNA & hairpin RNA \\
\hline IPP & isopentenyl pyrophosphate \\
\hline ITR & itraconazole \\
\hline LB & Luria-Bertani medium \\
\hline Mal & maltose \\
\hline MEA & malt extract agar medium \\
\hline MEP & methylerythritol phosphate \\
\hline miRNA & micro interfering RNA \\
\hline MOPS & 3-(N-morpholino) propanesulfonic acid \\
\hline MVA & mevalonate pathway \\
\hline $\mathrm{Na}-\mathrm{ac}$ & sodium acetate \\
\hline NCBI & National Center for Biotechnology Information \\
\hline
\end{tabular}




$\begin{array}{ll}\text { OD } & \text { optical density } \\ \text { PBS } & \text { phosphate buffered saline } \\ \text { PCR } & \text { polymerase chain reaction } \\ \text { PEG } & \text { polyethylene glycol } \\ \text { PI } & \text { phagocytic index } \\ \text { PMC } & \text { PEG - sorbitol - MOPS - calcium chloride (buffer) } \\ \text { qPCR } & \text { quantitative real-time PCR } \\ \text { RPM } & \text { revolutions per minute } \\ \text { SMC } & \text { sorbitol - MOPS - calcium chloride (buffer) } \\ \text { SIM } & \text { simvastatin } \\ \text { siRNA } & \text { small interfering RNA } \\ \text { SZMC } & \text { Szeged Microbiology Collection, Hungary } \\ \text { TAE } & \text { tris - acetic acid - disodium EDTA (buffer) } \\ \text { TF } & \text { transcription factor } \\ \text { Treh } & \text { trehalose } \\ \text { TRIS } & \text { tris(hydroxymethyl)aminomethane } \\ \text { YNB } & \text { yeast nitrogen base } \\ \text { YPG } & \text { yeast extract - pepton - glucose medium }\end{array}$

Mucor circinelloides genes and encoded proteins frequently occur in the thesis:

$\begin{array}{ll}\begin{array}{l}\text { hmgS } \\ m v k\end{array} & \begin{array}{l}\text { HMG-CoA synthase } \\ \text { mevalonate kinase } \\ \text { diphosphomevalonate decarboxylase } \\ \text { (pyrophosphomevalonate decarboxylase or mevalonate 5- } \\ \text { pyrophosphate decarboxylase) }\end{array} \\ & \text { isopentenyl pyrophosphate (IPP) isomerase } \\ \text { ipi } & \text { farnesyl pyrophosphate (FPP) synthase } \\ \text { isoA } & \text { geranylgeranyl pyrophosphate (GGPP) synthase } \\ l e u A & \alpha \text {-isopropylmalate isomerase } \\ \text { pyrG } & \text { orotidine 5'-monophosphate decarboxylase } \\ \text { gpd1 } & \text { glyceraldehyde-3-phosphate dehydrogenase 1 } \\ z r t 1 & \text { ZIP zinc transporter }\end{array}$




\section{OVERVIEW}

Members of the subphylum Mucoromycotina, order Mucorales (such as Lichtheimia, Mucor, Rhizomucor and Rhizopus species) are saprotrophic fungi, which also have medical, industrial, biotechnological and agricultural importance. Some species may cause post-harvest damage in agriculture; or are used as producers of extracellular enzymes, organic acids and carotenoids. Several species belonging to this fungal group are also considered opportunistic human pathogens, which can cause fatal systemic infections (zygomycosis or mucormycosis) in immunocompromised patients with neutropenia, diabetic ketoacidosis or serious skin injuries.

Metabolites synthesized via the mevalonate-isoprenoid pathway (such as sterols, functional groups of proteins and carotenoids) play an important role in signal transduction, morphogenesis, apoptosis, adaptation to environmental change and protection against free radicals. Today ergosterol and its biosynthetic pathway are the major targets of the antifungal agents used in clinics to treat infections caused by Mucoromycotina fungi. The therapy of mucormycosis is still limited because of the intrinsic resistance of these fungi to the majority of the currently clinically applied antimycotics. To date limited information is available about the function and regulation of the mevalonate-isoprenoid biosynthesis pathway genes in Mucoromycotina fungi. Investigation of the fungal mevalonateisoprenoid biosynthesis pathway may allow the identification of new potential novel drug targets that could bolster the arsenal of available options to treat fungal infections. Furthermore it provides a great opportunity to develop new therapeutic strategies with special attention paid to biosynthesis pathway that promote growth inhibition of fungi. Moreover modification of the pathway may allow to isolate $\beta$-carotene overproducing mutant strains. Thus, our aim was to characterize six genes of the Mucor circinelloides mevalonate-isoprenoid pathway, encoding the HMG-CoA synthase ( $h m g S)$, mevalonate kinase $(m v k)$, diphosphomevalonate decarboxylase $(d m d)$, isopentenyl pyrophosphate isomerase (ipi), farnesyl pyrophosphate synthase (isoA) and geranylgeranyl pyrophosphate synthase $(\operatorname{car} G)$. Farnesyl pyrophosphate and geranylgeranyl pyrophosphate serves as precursors of sterols and carotenoids, respectively. 


\section{INTRODUCTION}

\subsection{Characterization and taxonomy of Mucoromycotina fungi}

The phylum formerly known as Zygomycota was an artificial assemblage of fungi of uncertain taxonomic position. In a previous classification the phylum consisted of two classes, zygomycetes and trichomycetes (Benny et al., 2001) and is composed of fungi that form coenocytic mycelia and reproduce sexually by the production of zygospores (Whittaker, 1969; Tanabe et al., 2004, 2005). Hibbett and coworkers abandoned the phylum Zygomycota and classified zygomycete fungi into four subphyla, including Entomophthoromycotina, Kickellomycotina, Mucoromycotina, and Zoopagomycotina and phylum Glomeromycota (Hibbett et al., 2007). In addition, four years later based on a multigene analyzis, a new subphylum Mortierellomycotina was also described, which consisting Mortierellales species (Hoffmann et al., 2011). A new phylogenetic classification follows the principles promoted by Hibbett and coworkers and two phyla, Mucoromycota and Zoopagomycota was circumscribed (Hibbett et al., 2007; Spatafora et al., 2016). The two phyla consist of six subphyla, four classes, and 16 orders. Zoopagomycota comprises Entomophthoromycotina (with classes Entomophthoromycetes, Basidiobolomycetes and Neozygitomycetes), Kickxellomycotina and Zoopagomycotina (previous orders Eccrinales and Amoebidiales have been demonstrated not to be members of Kingdom Fungi), while Mucoromycota comprises Glomeromycotina (with class Glomeromycetes), Mortierellomycotina, and Mucoromycotina subphyla (Fig. 1, Benny and O'Donnell, 2000; Cafaro, 2005; Spatafora et al., 2016). All taxa are either demonstrated or presumed to be monophyletic. Former phylogenetic analyzes suggested that the phylum Zygomycota is polyphyletic or paraphyletic, now Spatafora and coworkers demonstrated that the two clades, Zoopagomycota and Mucoromycota, form a paraphyletic grade from which Dikarya are derived (Richardson, 2009; Spatafora et al., 2016).

Most of the genera of the former phylum are currently included in Mucoromycotina, being the subphylum with the highest number of species (Hibbett et al., 2007; Spatafora et al., 2016). Mucoromycotina includes Mucor, Rhizopus, and the majority of the most common and best known zygomycetes. Fungi belonging to the subphyla are fast-growing, filamentous, non-flagellated fungi, which form coenocytic mycelia (lacking regular septation; septas are usually formed in specialized hyphae playing role in reproduction) and cell wall consisting of high level of chitin and chitosan, low level of glucan and fucose (a carbohydrate present only in few fungal taxa) (Richardson, 2009; Mélida et al., 2015; 
Araújo and Hughes, 2016; Muszewska et al., 2018). These fungi are mainly saprobes, and it is frequently isolated from soil, dung, plant debris, and sugar-rich plant parts (e.g. fruits), but mycoparsasites, ectomycorrhizal species are also represented in the subphyla (Spatafora et al., 2016).

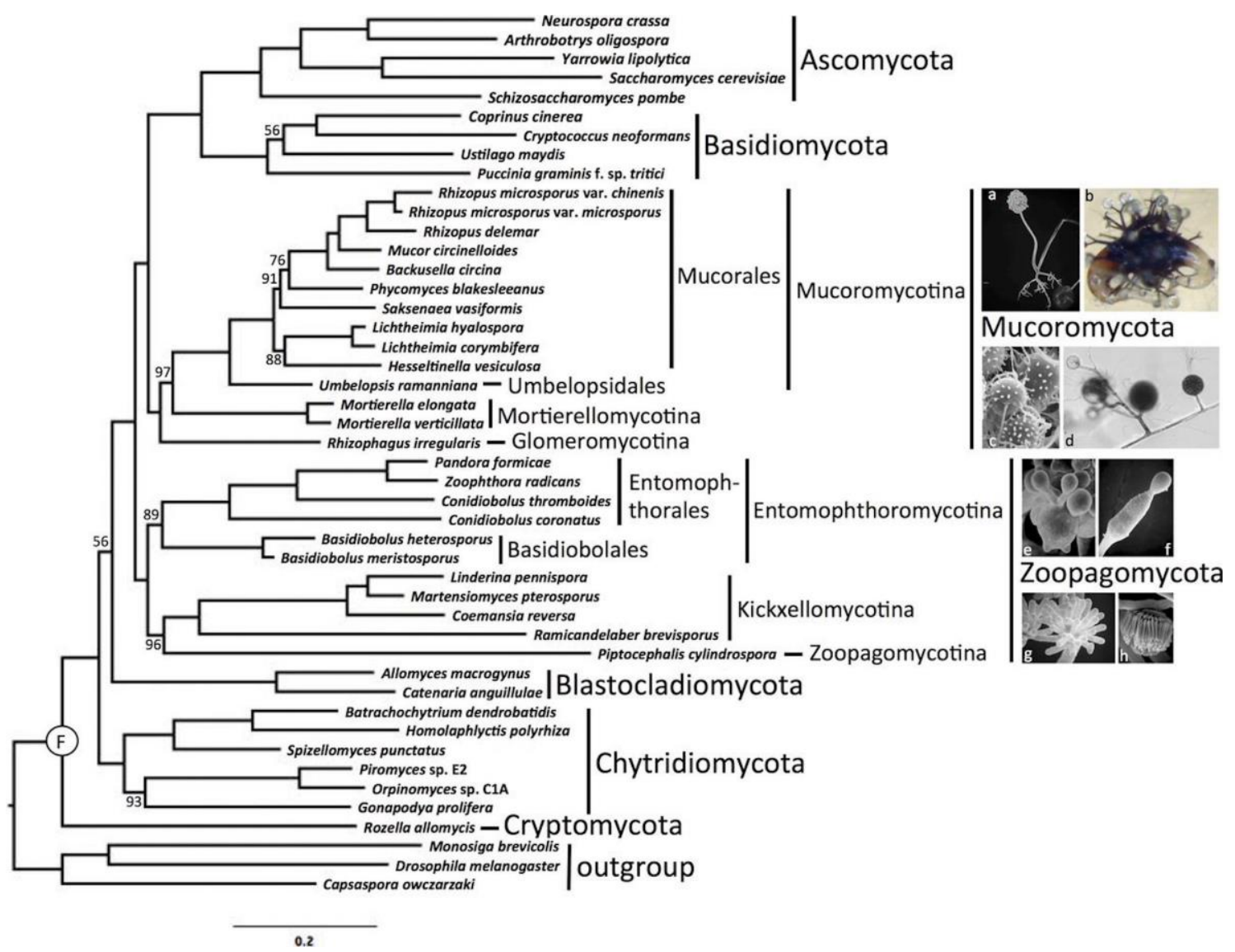

Fig 1. Phylogenetic tree of Kingdom fungi based on the concatenated alignment of 192 conserved orthologous proteins (Spatafora et al., 2016).

Mucoromycotina fungi have a haploidic life cycle; the only diploid cells are zygospores (Eslava et al., 1975; James and Kerry, 2004). Reproduction normally happens by asexually, which typically involves the production of sporangia (Fig. 2) and/or sporangioles at the end of sporangiophores (specialized hyphae) and uni- or multinucleate sporangiospores (Schachtschabel et al., 2008). Sporangiospores are typically dispersed with water or wind. Sexual reproduction takes place with zygospores, which are formed by gametangial conjugation (Fig. 2) (Eslava et al., 1975; James and Kerry, 2004). Homothallic (conjugation takes place between hyphae of same strain) and heterothallic (conjugation occurs between hyphae belong to the opposite mating type strains) species are 
also occurring in the subphyla (Idnurm, 2011). The sporangium rises from zygospore bearing haploid sporangiospores (Fig. 2) (Eslava et al., 1975; James and Kerry, 2004).

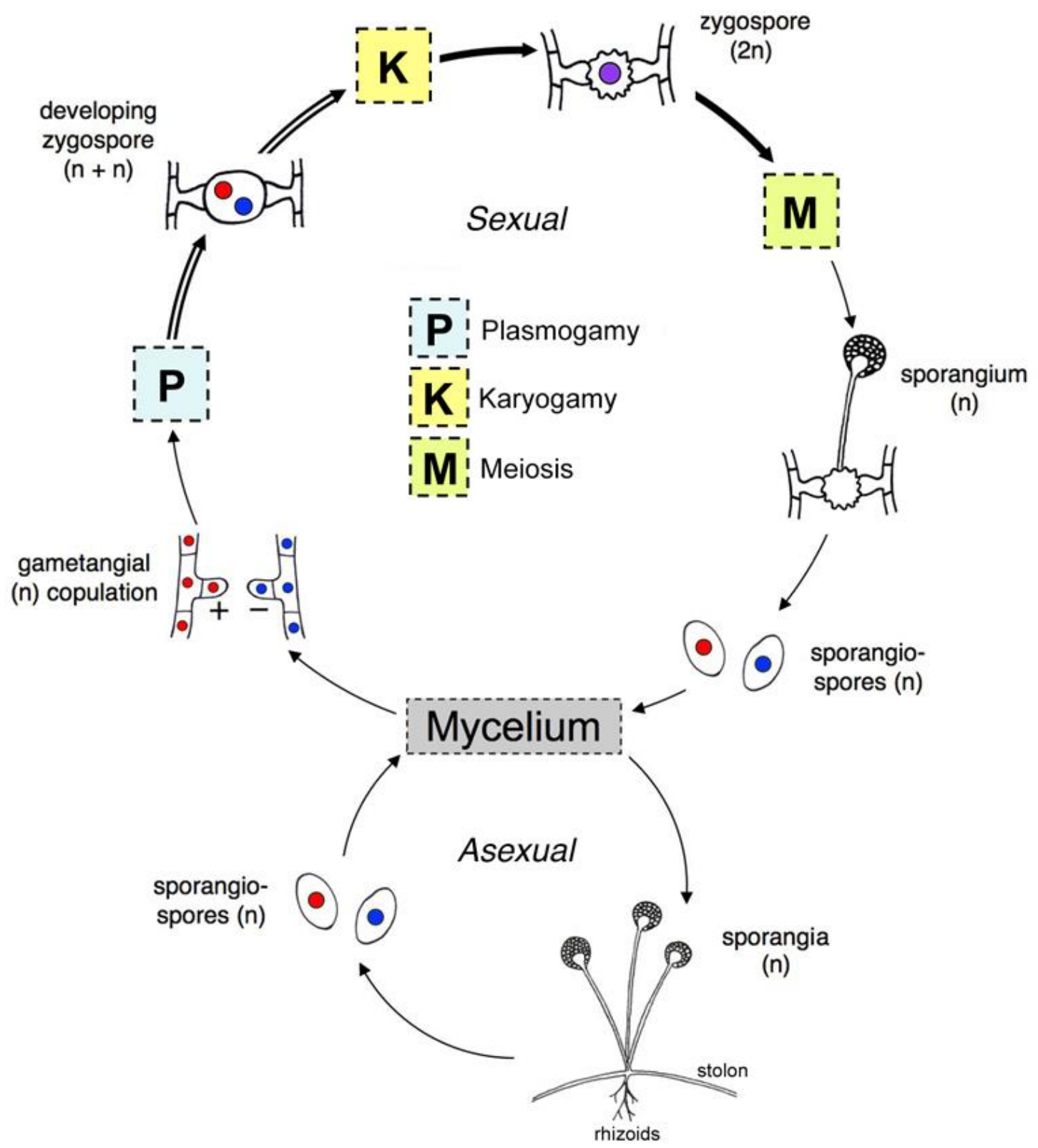

Fig 2. Reproduction cycles of Mucoromycotina fungi (Carris et al., 2012).

\subsection{Economic and clinical importance of Mucoromycotina fungi}

Fungal secondary metabolites are utilized as antibiotics, toxins, pesticides, and animal as well as plant growth factors (Nielsen and Nielsen 2017). Mucoromycotina fungi are recieving a growing attention due to their evolutionary distance to other fungi (Gladieux et al., 2014). The role of these fungi in industry, like production of 
polyunsaturated fatty acids, beer and ethanol with Mucor indicus, degradation of oil waste, removal of heavy metal from waste water (biosorption) with $M$. indicus and biotransformation with Mucor rouxii are described well (Arcidiacono et al., 1992; Harms et al., 2011; Karimi and Zamini, 2013; Simister et al., 2015). Mucoromycotina fungi are also used as producers of organic acids (e.g. lactic, malic and fumaric acid) and hydrolytic enzymes, in the elaboration of different kinds of Asian food and in cheese ripening, and Blakeslea trispora for $\beta$-carotene production (Shetty et al., 2000; Abe et al., 2004; Millati et al., 2005; Karimi and Zamini, 2013). $\beta$-carotene is a fat soluble pigment that has great commercial value due to its diverse uses in food, pharmaceutical products, cosmetics and textiles (Hussein et al., 2006; Britton et al., 2009; Sandmann, 2015). Carotenoids are widely used as natural pigments mostly because of their antioxidant properties (Bhosale and Bernstein, 2005). They protect cells against photooxidation by quenching singlet oxygen, free radicals (e.g. prevention of lipid peroxidation) and reactive oxygen species (Bhosale and Bernstein, 2005).

The worldwide carotenoid market value was $\$ 1.5$ billion in 2014 and is assumed to reach nearly $\$ 1.8$ billion in 2019 , with a compound annual growth rate of $3.9 \%$ reported by global market during 2015 (global market for carotenoids (2015)). Microbial $\beta$-carotene currently produced by the industry with alga Dunaliella salina or B. trispora (Lamers, 2008; Papaioannou and Liakopoulou-Kyariakides, 2012). The wild-type $B$. trispora produces carotenoids in similar amount to $M$. circinelloides, but transformation systems are not available for genetic modification to improve carotenoid production. Mixed culture of different mating types of $B$. trispora and optimized fermentation media are used industrially for carotenoid production (Mehta et al., 2003). In contrast, approach for efficient genetic transformation of $M$. circinelloides, such as overexpression and gene silencing, allow us to improving carotenoid production (Csernetics et al., 2011; TorresMartínez et al., 2012).

Several members of Mucoromycotina and Entomophthoromycotina are considered opportunistic human pathogens; and these infections are called mucormycosis and entomophthoromycosis, respectively (formerly zygomycosis) (Kwon-Chung, 2012; Riley et al., 2016). Species of the former class Zygomycetes were firstly reported to cause disease in humans in a publication entitled Mycosis Mucorina (Platauf, 1885). This first case of disseminated disease has presented in a patient suffering from cancer caused by Lichtheimia (formerly Absidia) corymbifera (Platauf, 1885). Mucor and Lichtheimia are, after Rhizopus, the clinically most relevant genus of Mucoromycotina (Ribes et al., 2000; 
Alvarez et al., 2009). In addition, species of Rhizomucor, Apophysomyces, Saksenaea, Cunninghamella, Cokeromyces, and Syncephalastrum have also been described as caustaive agents of mucormycosis, but less cases have been reported (Gomes et al., 2011).

Five major forms of mucormycosis occur, including rhino-orbito-cerebral, pulmonary, disseminated, cutaneous, and gastrointestinal, while species of Entomophthoromycotina generally cause locally manifested - cutaneous and subcutaneous and slowly progressive infections (Prabhu and Patel, 2004; Pfaller and Diekema, 2005). An increase in the number of mucormycosis has been observed in the last few decades, mainly because of the modern surgical interventions, immunosuppressive therapies and irresponsible use of antimicrobial agents (Ribes et al., 2000; Chayakulkeeree, et al., 2006; Ibrahim et al., 2012). According to Centers for Disease Control and Prevention USA (CDC, 2015) the overall mortality rate of mucormycosis found to be $54 \%$. It was $46 \%$ among people with sinus infections, $76 \%$ for pulmonary infections, and $96 \%$ for disseminated mucormycosis $(\mathbf{C D C}, \mathbf{2 0 1 5})$. The most vulnerable group of these infections are immunocompromised patients, the major risk factors include neutropenia, cancer, corticosteroid treatment, diabetes mellitus in ketoacidosis, deferoxamine treatment, organ transplantation, and burn injury (Sugar, 2000; Sugar and Liu, 2000; Prabhu and Patel, 2004; Ibrahim et al., 2008). Phagocytes - primarily macrophages and neutrophils - have the most important role in host immune defence against fungi causing mucormycosis, thus longer neutropenia is one of the major risk for developing infection (Ibrahim et al, 2012; Morace and Borghi, 2012). Pulmonary alveolar macrophages play important role in defence against these fungi, because inhalation of the sporangiospores is one of the most common way of infection (van de Veerdonk et al., 2010).

\subsection{Antifungal agents applied in clinics to treat fungal infections}

Only few antimycotic drugs were reported which are able to kill the Mucoromycotina fungi, whereas others merely only inhibit the growth of pathogens. The proper antifungal therapy and selection of drug should be based on several criteria, such as immune capability of the host, site of infection, characteristics of the infection (the fungal species and its susceptibility to different antifungal drugs), and pharmacokinetic characteristics of the antifungal drug (e.g., absorption, elimination, and toxicity) (Lepak et al., 2015). Mucorales are mostly resistant against widely used antifungal drugs used in clinics (such as against different azoles and echinocandins), thus generally, antifungal therapy need to be combined with surgical debridement of the necrotic regions. 
Today, ergosterol and its biosynthetic pathway are the major targets of antifungal therapy used to treat infections caused by Mucoromycotina fungi. Based on the mode of action the drugs belong to different classes, which are as follows: (1) polyene macrolides (e.g. Amphotericin B, (AmB); Fig. 3); (2) azole derivatives (such as ketoconazole, fluconazole, itraconazole (ITR), posakonazole, clotrimazole (CLO) and voriconazole; Fig. 3); (3) allylamines (e.g. terbinafine); (4) DNA and RNA synthesis inhibitors (flucytosine); (5) echinocandins (Selvakumar et al., 2006; Vandeputte et al., 2012; Bondaryk et al., 2013).

Ergosterol is the most prevalent sterol in the fungal plasma membrane. The target of polyene macrolides (produced by Streptomyces spp.) is ergosterol; through the channels formed by the complex cell leaks $\mathrm{K}^{+}$, resulting in a disruption of the proton gradient (Lemke et al., 2005). In higher concentrations, polyenes also inhibit chitin syntheses, moreover AmB causes oxidative damage to plasma membranes (Sudoh et al., 2000). AmB (Fig. 3) is effective against a wide variety of fungi, including yeasts and molds such as Mucoromycotina fungi (Lemke et al., 2005). Treatment with AmB is often followes by nephrotoxicity rather than therapeutic efficacy; whereas lipid formulations have reduced toxicity and it allows higher therapeutic doses (Azanza et al., 2015).

Azoles act as inhibitors of the cytochrome-P450 dependent lanosterol 14- $\alpha$ demethylase, a key enzyme in ergosterol biosynthesis (Vandeputte et al., 2012). The active binding site of lanosterol 14- $\alpha$ demethylase contains a heme domain (Sheng et al., 2009). Azoles bind with a particular nitrogen molecule in the azole ring core to the iron atom of the heme domain, preventing the demethylation of lanosterol (Joseph-Horne and Hollomon, 1997; Sheng et al., 2009). Azoles may also interact with the 3-ketosteroid reductase, an enzyme in methylsterol biosynthesis (Sant et al., 2016). Azoles used in high dosage may cause azole-induced toxic injury (Lo $\operatorname{Re} \mathbf{V} 3^{\text {rd }}$ et al., 2016). The first azole accessible for systemic use was CLO (Fig. 3), however, its application was restricted due to inconsistent concentrations in the blood serum. Miconazole is effective to treat systemic infections, but it could be given only intravenously. ITR, the first triazole used in humans, is available for oral (capsules and cyclodextrin solution to enhance the bioavailability of the drug) and intravenous administration (Carrier et al., 2007). Azoles are used for prophylaxis in transplant patients to prevent invasive candidiasis, aspergillosis and mucoromycosis (Ibrahim et al., 2012). Several Mucoromycotina fungi shows resistance to the most of the widely used azoles (Caramalho et al., 2017). Nowadays, ITR (Fig. 3), posaconazole and isovuconazole are used frequently to treat mucormycosis, while for 
combination therapy azoles are using mainly with $\mathrm{AmB}$ (Greenberg et al., 2006; Cornely et al, 2013; Pagano et al., 2013; Caramalho et al., 2015; Marty et al., 2016).
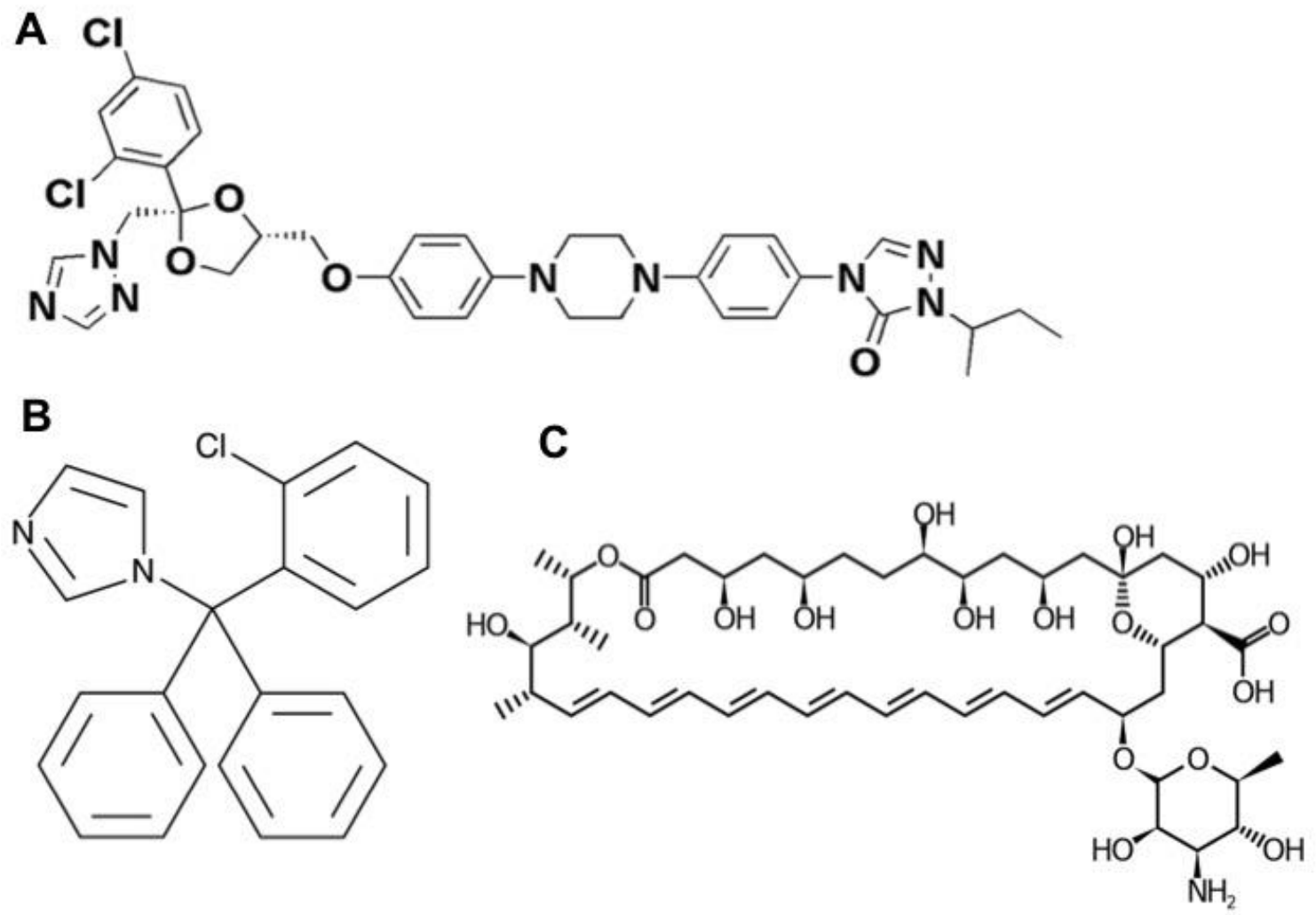

Fig 3. Biochemical structure of antifungal agents: (A) itraconazole, (B) clotrimazole and (C) AmphotericinB (Warrilow et al., 2010; Chávez-Fumagalli et al., 2015).

Echinocandins (such as micafungin, anidulafungin and caspofungin) are water soluble lipopeptides, which inhibit the $\beta(1-3)$-D-glucane synthase, thus reducing the cell wall integrity. No any toxic effect of these agents had been described. Echinocandins are used only parentheral, they have fungicidal activity against Candida and Aspergillus species, while Mucorales shows resistance to these antifungals (Walker et al., 2010).

Allylamins are inhibiting the squalene epoxydase, enzyme involved in the ergosterol biosynthesis. Mainly used topically against dermathophytes, also effective against Candida albicans (Georgopapadakou et al., 1992).

Flucytosine act as inhibitor of the RNA and DNA synthesis in fungi. As the effect of cytosine deaminase, 5-fluorocytosine is converted to cytostatic 5-fluorouracil, which next convert into the RNA intercalating, thus protein synthesis inhibitior, 5-fluorouridine triphosphate and 5-fluorodeoxyuridine monophosphate. The latter inhibit the thymidylate synthase, and deoxythymidine triphosphate (dTTP) synthesis, thereby the DNA synthesis. 
It is generally used in combination with $\mathrm{AmB}$ in treatment of Cryptococcus infections (Dixon and Walsh, 1996; Loyse et al., 2013).

Microorganisms develop the system to prevent the fungicidal or fungistatic effect of antifungals drugs, that is classified into three basic mechanisms, (i) try to reduce the uptake and accumulation of the drug inside the fungal cell, (ii) reducing the affinity of the drug at the target point, and (iii) moderation of metabolism to counterbalance the antifungal drug effect (Sanglard, 2002). Moreover in high degree resistant of clinical isolates, combination of several mechanisms was also observed (Perea et al., 2001). Expanding in resistance with antifungal therapy is due to the sequential acquisition of different mechanisms (Franz et al., 1998; Marr et al., 1998; MacCallum et al., 2010). The cause of resistance to polyenes has been studied, and is a significant alteration of the lipid composition in the plasma membrane (Kelly et al., 1994; Lemke et al., 2005). This may be one of the reasons to a lower affinity of AmB, probably a lack of the binding site (Joseph et al., 1995). Another reason for $\mathrm{AmB}$ protection might be a modified substance of $\beta-1,3$ glucans in the cell wall. These components, which increase the stability of the cell wall, influence the access of large molecules such as AmB to the plasma membrane (Seo et al., 1999; MesaArango et al., 2016).

The susceptibility of $M$. circinelloides against wide range of antifungal agents and statins were tested and minimal inhibitory concentrations (MIC) were determined (Table 1). The MIC of AmB was determined to $M$. circinelloides in range of 0.06 to $1.0 \mu \mathrm{g} / \mathrm{ml}$, while differences were observed in the susceptibility against different azoles, for example M. circinelloides showed resistance against fluconazole, voriconazole and ravuconazole, but posaconazole, ITR, and ketoconazole inhibited the growth of the fungus in the investigated concentrations (Table 1). M. circinelloides also showed sensitivity to lovastatin and were resistant to different echinocandins (Table 1). It has to be noted that different strains belonging to the same species shows high deviation in susceptibility to antifungals; it can be also observed in case of $M$. circinelloides, resulting in large scale of MIC, such as in case of posaconazole and ITR. Our research group previously determined the MIC of different antifungal agents against M. circinelloides MS12 strain (unpublished data), following the instructions of the Clinical and Laboratory Standards Institute (CLSI) M38-A2 (2008) protocol. The M. circinelloides MS12 showed resistance to echinocandins and most of the azoles involved in that study (such as fluconazole, posaconazole, ITR, voriconazole, ketoconazole and econazole), but miconazole and CLO were able to inhibit it's growth in $16 \mu \mathrm{g} / \mathrm{ml}$ concentration. MIC for AmB was determined in $2 \mu \mathrm{g} / \mathrm{ml}$ and FLU 
and lovastatin were also able to inhibit the growth of $M$. circinelloides MS12 in $8 \mu \mathrm{g} / \mathrm{ml}$ and $64 \mu \mathrm{g} / \mathrm{ml}$, respectively (unpublished data).

Table 1. MIC of antimycotics and statins against $M$. circinelloides determined in different studies.

\begin{tabular}{|c|c|c|}
\hline Antimycotic & $\begin{array}{c}\text { MIC } \\
(\mu \mathrm{g} / \mathrm{ml})\end{array}$ & References \\
\hline Amphotericin B & $0.06-1$ & $\begin{array}{c}\text { Caramalho et al. 2015; Almyroudis et al. 2007; Drogari-Apiranthitou et al. 2012; } \\
\text { Salas et al. 2012 }\end{array}$ \\
\hline Posaconazole & $0.50-32$ & $\begin{array}{c}\text { Caramalho et al. 2015; Almyroudis et al. 2007; Drogari-Apiranthitou et al. 2012; } \\
\text { Salas et al. 2012 }\end{array}$ \\
\hline Itraconazole & $2->8$ & Almyroudis et al. 2007; Drogari-Apiranthitou et al. 2012 \\
\hline Ketoconazole & 16 & Almyroudis et al. 2007 \\
\hline Fluconazole & $>64$ & Almyroudis et al. 2007 \\
\hline Voriconazole & $>8$ & Drogari-Apiranthitou et al. 2012 \\
\hline Ravuconazole & $>8$ & Drogari-Apiranthitou et al. 2012 \\
\hline Terbinafine & 8 & Drogari-Apiranthitou et al. 2012 \\
\hline Anidulafungin & $>8$ & Drogari-Apiranthitou et al. 2012 \\
\hline Caspofungin & $>16$ & Almyroudis et al. 2007 \\
\hline Lovastatin & $5-40$ & Galgóczy et al. 2011 \\
\hline Fluvastatin & $>25$ & Galgóczy et al. 2011 \\
\hline
\end{tabular}

\subsection{The mevalonate-isoprenoid biosynthesis pathway in M. circinelloides}

Isoprenoids (or terpenoids) are a group of functionally diverse compounds comprising of at least 50,000 different structures mostly identified from plants, bacteria and fungi (Hemmerlin et al., 2012; Schmidt-Dannert, 2015). These metabolites (such as carotenoids, ergosterol and functional groups of farnesylated and geranylgeranylated proteins) have several biological function: they are playing role in morphogenesis, signal transduction, apoptosis, protection against free radicals, adaptation to environmental change and contribute to membrane permeability and integrity (Wawrzyn et al., 2012). In fungi, metabolites are synthesized via the mevalonate-isoprenoid biosynthesis pathway, in which formation of mevalonate is one of the key steps.

In fungi the mevalonate pathway (MVA) begins with acetyl-CoA, which is the final metabolite of the glycolysis pathway. Three molecules of acetyl-CoA are sequentially condensed by thiolase and 3-hydroxy-3-methylglutaryl-CoA (HMG-CoA) synthase to form HMG-CoA, which is then reduced to mevalonate by HMG-CoA reductase, expected that it 
might work is a rate-constraining advance of this pathway (Wang and Keasling, 2002; Burg et al., 2011; Liao et al., 2016). Next, conversion of mevalonate to isopentenyl pyrophosphate (IPP) is catalyzed by three enzymes. In this part of the biosynthesis mevalonate is phosphorylated at the $5-\mathrm{OH}$ position by mevalonate kinase and mevalonate5-phosphate kinase, and then decarboxylated to IPP by diphosphomevalonate decarboxylase (mevalonate-5-diphosphate decarboxylase) (Fig. 4) (Miziorko, 2011; Liao et al., 2016). Recently, two atypical types of that part of the MVA pathway have been described in Haloferax volcanii and Thermoplasma acidophilum (Dellas et al., 2013). One involves the decarboxylation of mevalonate-5-phosphate to isoprenyl phosphate (IP) by mevalonate-5-phosphate decarboxylase and followed by the phosphorylation of IP to IPP by IP kinase (Grochowski et al., 2006; Dellas et al., 2013). The other involves phosphorylation of mevalonate at both ends (at the 3-OH and 5-OH positions) by mevalonate-3-kinase and mevalonate-3-phosphate-5 kinase to generate mevalonate-3,5bisphosphate, which is decarboxylated to IPP by mevalonate-5-phosphate decarboxylase (Azami et al., 2014; Vinokur et al., 2014).

In the isoprenoid pathway, formation of dimethylallyl pyrophosphate (DMAPP) is catalyzed by IPP isomerase, and condensation of IPP and DMAPP form the intermediate geranyl pyrophosphate (GPP). The carbon chain is then increases by repetitive condensations with further IPP units. These steps are managed by prenyltransferases, such as farnesyl pyrophosphate and geranylgeranyl pyrophosphate (FPP and GGPP, respectively) synthases, and produce linear intermediate compounds with various lengths (Fig. 4) (Chang et al., 2013). The compounds formed via the isoprenoid pathway serve as precursors in many different biosynthetic side routes leading to the formation of several types of end products, such as carotenoids, ergosterol, terpenoid moiety of ubiquinones and prenyl groups of certain proteins (Liang et al., 2002; Wawrzyn et al., 2012).

Carotenoids belong into the big and diverse family of isoprenoid compounds. The carotenoid biosynthesis starts with the condensation of two molecules of C20 GGPP units, thus formation of phytoene. Several dehydrogenation and two cyclisation steps result in $\beta$ carotene (Fig. 5) - the major carotenoid produced by most of the Mucoromycotina fungi catalyzed by phytoene synthase/lycopene cyclase (a bifunctional enzyme) and phytoene dehydrogenase, which encoded by carRP and carB genes, respectively, in $M$. circinelloides (Velayos et al., 2000a, 2000b). Conversion of $\beta$-carotene to xanthophylls ( $\beta$-carotene with functional groups containing oxygen, such as zeaxanthin and $\beta$-cryptoxanthin) was also described in M. circinelloides (Papp et al., 2006, 2013; Csernetics et al., 2011). Induction 
of carotenoid production by light to be accounted as conserved in fungi, such as in Neurospora crassa, Phycomyces blakesleeanus and M. circinelloides (Silva et al., 2006; Zhang et al., 2016). In $N$. crassa $w c-1$ and $w c-2$ genes, encoding white-collar proteins (photoreceptors), play important role in light induction of carotenoid production (Cheng et al., 2003; He and Liu, 2005). Three homologos of $w c-1$ have been reported in $M$. circinelloides and one of them, $m c w c-1 c$, was also found to be involved in the light induction of carotenoid production (Silva et al., 2006).

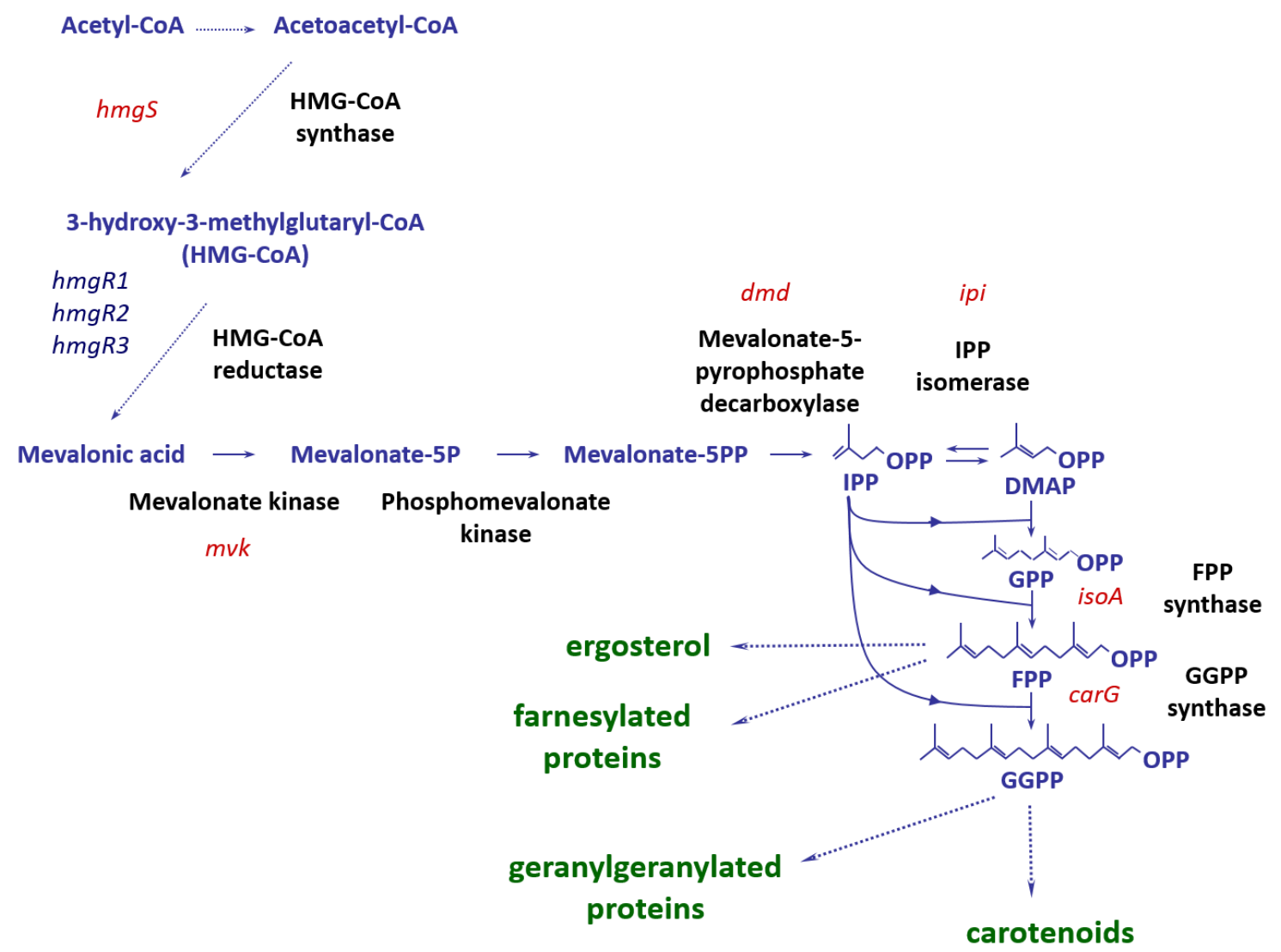

Fig 4. The mevalonate-isoprenoid biosynthesis pathway in M. circinelloides. The identified genes involved in the biosynthesis are shown with red and dark blue (Csernetics et al., 2011; Nagy et al., 2014 and unpublished data). IPP - isopentenyl pyrophosphate, DMAPP - dimethylallyl pyrophosphate, GPP - geranyl pyrophosphate, FPP - farnesyl pyrophosphate, GGPP geranylgeranyl pyrophosphate.

Sterols are neutral lipids of eukaryotic cells (Dupont et al., 2012). Three predominant forms represent the main sterols found in eukaryotes like cholesterol in vertebrates, phytosterols (sitosterol, stigmasterol, campesterol) in plants, and ergosterol in fungi (Fig. 5). While even structural and function studies have failed to show any 
advantages of ergosterol in comparision to cholesterol, moreover biosynthesis require more energy than cholesterol, the particular reason why ergosterol is present in fungi is still not clear (Shrivastava and Chattopadhyay, 2007; Weete et al., 2010).

Ergosterol is involved in several biological functions such as maintenance of cell membrane integrity and fluidity, furthermore in regulation and control of the cell cycle (Alvarez et al., 2007; Abe et al., 2009). Ergosterol biosynthesis is an oxygen-dependent process, as several enzymes of the post-squalene pathway require molecular oxygen (Rosenfeld and Beauvoit, 2003). In ergosterol biosynthesis pathway around 20 enzymes exist (Ferreira et al., 2005; Alcazar-Fuoli et al., 2008). As a result of a lack of ergosterol and/or accumulation of its precursor, the FgERG4 deletion mutant Fusarium graminearum displayed several defects in plasma membrane function, such as increased sensitivity to metal cations and osmotic stresses (Long et al., 2017).

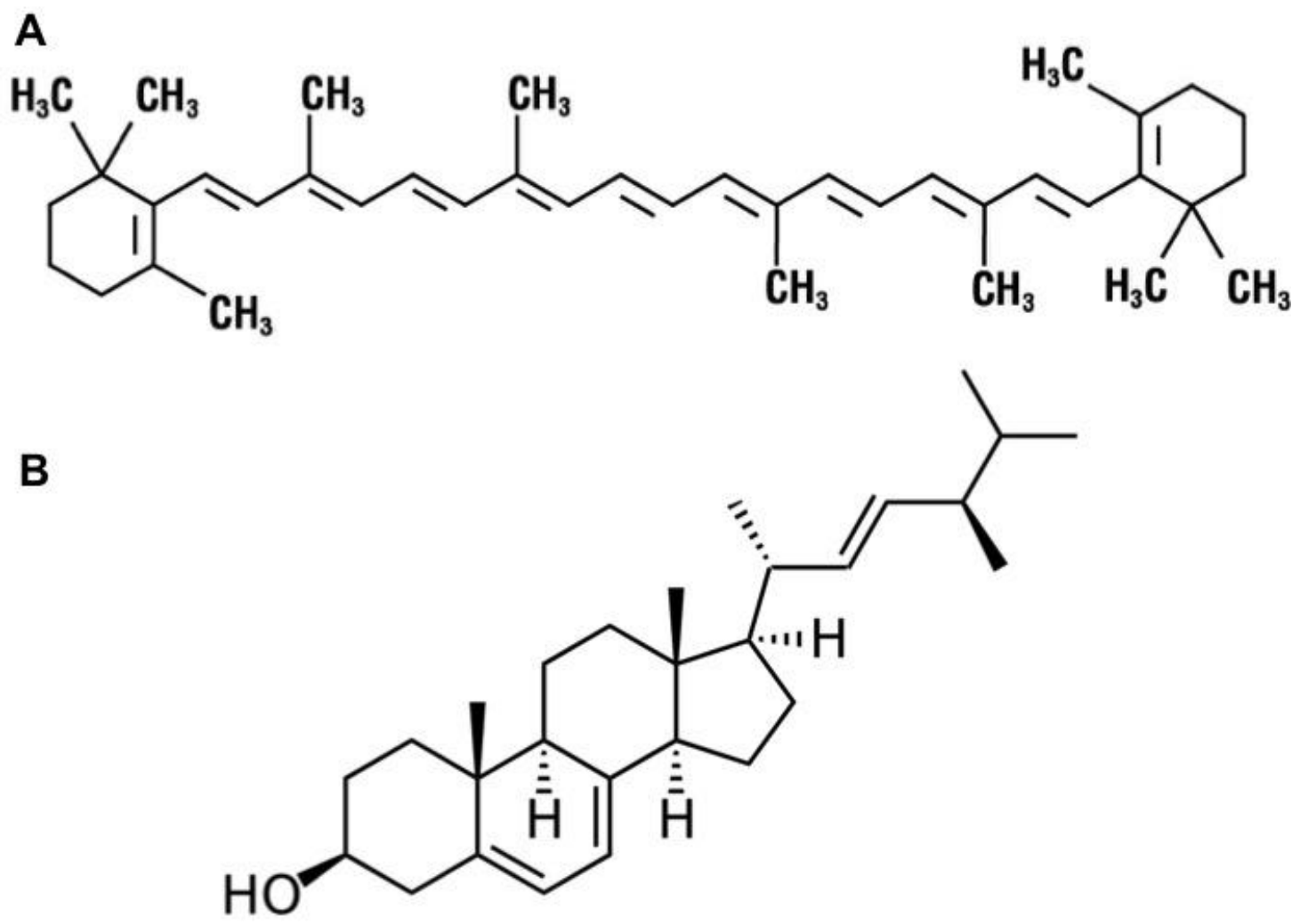

Fig 5. Biochemical structure: (A) $\beta$-carotene and (B) ergosterol (Valcarce et al., 2001; Oh et al., 2013).

Statins (such as lovastatin, fluvastatin (FLU), simvastatin (SIM), rosuvastatin and atorvastatin, Fig. 6) are cholesterol-lowering drugs. Statins are competitive inhibitors of the HMG-CoA reductase by binding to the enzyme and displace their natural substrate 
(Maciejak et al., 2013). It was shown that statins have fungicidal activity, and also act synergistically with different antifungal agents (such as azoles) against yeasts, dermatophytes and clinically important Mucoromycotina species (Lukács et al., 2004; Gyetvai et al., 2006; Nyilasi et al., 2014). Statins were found to inhibit not only ergosterol, but other mevalonate-derived metabolite biosynthesis pathways, such as dolichol, heme-A, isopentenyl tRNA, carotenoids, ubiquinone, and siderophores, as well as protein prenylation (Oide et al., 2006; Buhaescu and Izzedine, 2007; Schrettl et al., 2007).
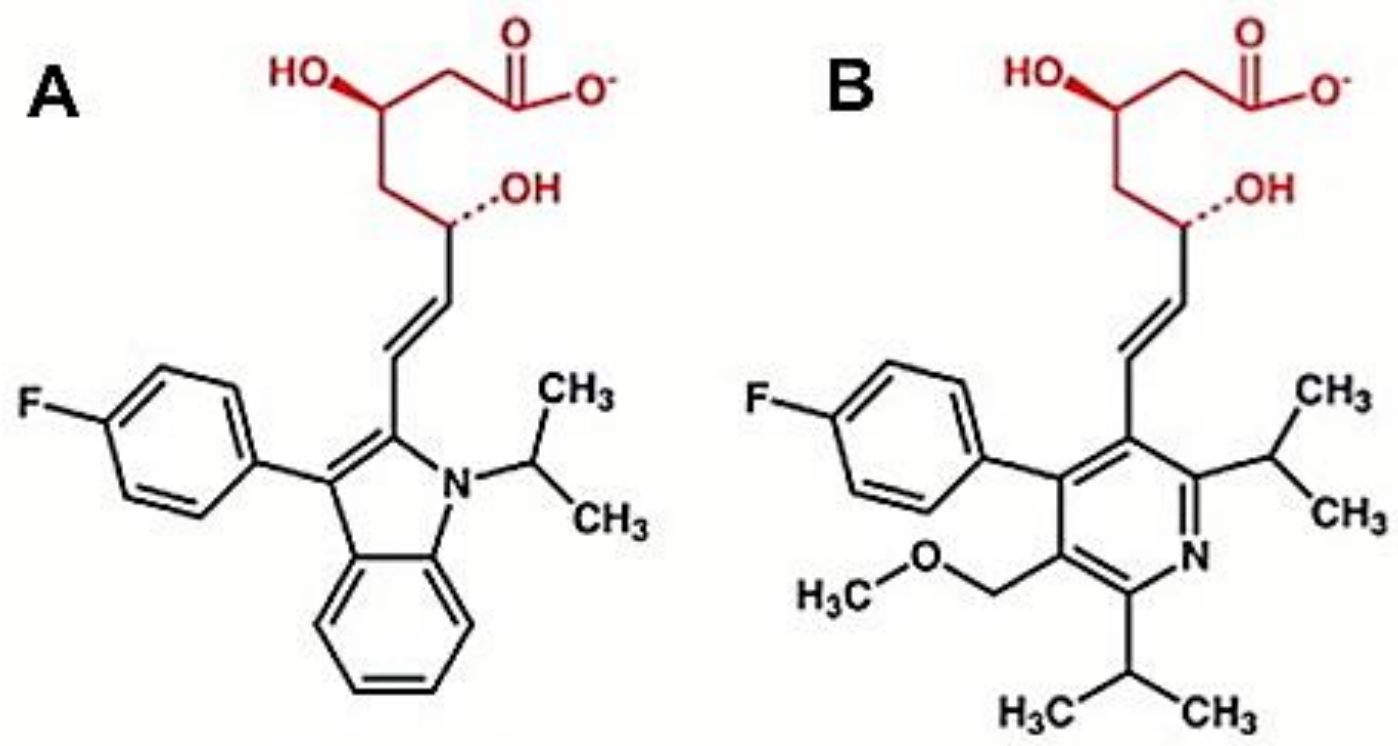

Fig 6. Biochemical structure: (A) simvastatin and (B) fluvastatin (Istvan et al., 2003).

\subsection{Genetic background of the mevalonate-isoprenoid pathway in $M$. circinelloides}

Several genes playing role in the mevalonate-isoprenoid biosynthesis in $M$. circinelloides have been cloned: $h m g S, m v k$ and $d m d$, encoding the HGM-CoA synthase, mevalonate kinase and diphosphomevalonate decarboxylase (unpublished data), the $h m g R l$, $h m g R 2$ and $h m g R 3$, encoding HMG-CoA reductases (Nagy et al., 2014), and the $i p i$, isoA and carG, encoding the IPP isomerase, FPP and GGPP synthases, respectively (Velayos et al., 2003, 2004; Csernetics et al., 2011).

The $h m g S, m v k$ and $d m d$ genes (unpublished data) have been cloned by our research group before the present thesis. The encoded enzymes are responsible for formation of HMG-CoA, mevalonate-5-phosphate and IPP, respectively. The HMG-CoA synthase - encoded by the 1812 bp (together with 256 bp 3'untranslated region) $h m g S$ gene) - consist of 452 aa, and the molecular weight is $50.6 \mathrm{kDa}$. Two catalytic domains were 
identified: the N-terminal (9 - 182 aa) and C-terminal (183 - 451 aa). Transcription factor (TF) binding sites in the promoter of $h m g S$ responsible for regulation of sterol biosynthesis have also been identified (such as SRE motifs, and NF-Y, Sp1, CREB sites). The mevalonate kinase - encoded by the 1364 bp $m v k$ gene - belongs to the enzyme family of galacto-, homoserine-, mevalonate- and phosphomevalonate (GHMP) kinases. The molecular weight of 409 aa protein is $44.9 \mathrm{kDa}$. The protein consists of one transmembrane domain (141 - 165 aa), thus it is anchored to the cell membrane. Several potential TF binding sites is presumed in the promoter: binding sites of ADR1 (positive regulators of peroxisomal genes in yeasts and PRARs ( $\alpha$-peroxisomal proliferator activated receptors (Bemis and Denis, 1988)). Binding sites of TFs mediating the expression of the HSF1 gene heat shock protien have also been identified (Harrison et al., 1994). Presumably these factors play role in regulation of ergosterol biosynthesis (Harrison et al., 1994; Palmer et al., 1995; Bemis and Denis, 1998). The diphosphomevalonate decarboxylase - encoded by

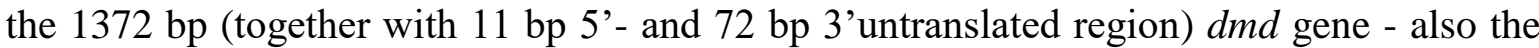
member of the GHMP enzyme family and consist of two GHMP kinase catalytic domains (108 - 166 aa and 240 - 224 aa). Molecular weight of the 410 aa protein is $45.2 \mathrm{kDa}$. In the promoter region TF binding sites of ADR1 and HSF1, moreover ABF2 and NIT-2 have also been identified (unpublished data).

The $h m g R 1, h m g R 2$ and $h m g R 3$ genes were identified in the $M$. circinelloides genome, encoding HMG-CoA reductases (Nagy et al., 2014). The length of the genes are 3740, 3595 and $3491 \mathrm{bp}$, while the encoded proteins are 1107, 1078 and 1115 aa, respectively. The calculated molecular mass of these proteins are 120.78, 118.45 and $120.71 \mathrm{kDa}$, respectively. HMG-CoA reductases are membrane anchored proteins and three main domains can be identified: the $\mathrm{N}$-terminal hydrophobic domain containing several transmembrane segments, and a sterol-sensing domain; a short linker region and the conserved C-terminal catalytic domain (which contain the HMG-CoA binding motif). The proteins shows $47-57 \%$ amino acid identity to each other (Nagy et al., 2014). Overexpression of the $h m g R 2$ and $h m g R 3$ genes increased the carotenoid and ergosterol content in $M$. circinelloides, and decreased the sensitivity to statins at various degrees, while in the case of $h m g R l$ no significant changes were found, suggesting differences in the role of the encoded proteins in isoprenoid biosynthesis (Nagy et al., 2014).

The IPP isomerase is responsible for the formation of DMAPP. It is encoded the ipi gene that was cloned and characterized in $M$. circinelloides in Csernetics and coworkers studies (Csernetics et al., 2011). The 910 bp gene encodes a 225 aa protein. The predicted 
molecular mass of the putative IPP isomerase is $26 \mathrm{kDa}$. In the catalytic region $(18-208$ aa) the conserved „NUcleoside DIphosphate linked to some other moiety X” domain and the conserved cysteine and glutamic acid residues ( $\mathrm{C}^{85}$ and $\mathrm{E}^{147}$, respectively), important components of the active site, were also identified. The FPP synthase is a prenyltransferase, responsible for the conversion of IPP to GPP and FPP (to date GPP synthase was only identified in plants, Szkopińska and Plochocka, 2005). The 352 aa FPP is encoded by the 1492 bp isoA gene in M. circinelloides (Velayos et al., 2004). The GGPP synthase is also a prenyltransferase and is responsible for the conversion of FPP to GGPP. The GGPP synthase is encoded by the 1461 bp in $M$. circinelloides (carG gene). The encoded 303 aa protein has a predicted molecular mass of $34.78 \mathrm{kDa}$ (Velayos et al., 2003). Three short APE-like (al-3 proximal element - like) sequences have been identified in the promoter, thus transcription of the gene is regulated by blue light (Velayos et al., 2003). Effect of overexpression of the ipi, isoA and $\operatorname{car} G$ on the carotenoid production of $M$. circinelloides was analyzed and was found that all three genes, but mainly overexpression of the carG, resulted significant increase in the total carotenoid content (Csernetics et al., 2011). The effect was more prominent, when the genes were placed under the control of the $M$. circinelloides glyceraldehyde-3-phosphate dehydrogenase 1 (gpdl) regulatory regions (Csernetics et al., 2011).

\subsection{Genetic modification of Mucormycotina fungi}

Genetic transformation is a biological process involving in the delivery and maintenance of exogenous DNA in the cell. The first transformation of fungi was carried out successfully 40 years ago with S. cerevisiae (Beggs, 1978; Hinnen et al., 1978), thereafter with $N$. crassa (Case et al., 1979), Aspergillus nidulans (Balance et al., 1983; Yelton et al., 1984) and $M$. circinelloides (van-Heeswijck and Roncero, 1984). These early examinations built up a fundamental protocol for protoplast transformation by incorporating them in concentrated of polyethylene glycol (PEG) in the presence of calcium ions followed by exposure to DNA. Potential impediments of PEG-mediated transformation are getting high concentration of viable protoplasts, low transformation efficiency and high rates of transient transformants. However, due to its simplicity in technical operation and equipment required, the PEG-mediated method remains the most commonly used one to conduct transformation in filamentous fungi. Additionally other methods, including Agrobacterium tumefaciens-mediated transformation (ATMT), electroporation and biolistic transformations were also developed for fungi, including 
Mucoromycotina species (Ruiz-Diez, 2002; Michielse et al., 2004; Nyilasi et al., 2005; Gutierrez et al., 2011; Li et al., 2017).

The basic procedure for PEG-mediated transformation contains three major steps: protoplast preparation, uptake of the transforming DNA, and protoplast regeneration. Protoplasts or spheroplasts can be prepared through digestion of the cell walls of mycelium or germinating spores using cell wall degrading enzymes, such as helicase or glusulase from snail stomach preparation, and cellulase, $\beta$-1,3-glucanase, chitinase, and driselase from Trichoderma or other fungal species (Fincham, 1989; Jung et al., 2000). The digestion is performed in an osmotic buffer containing sorbitol or high salt concentration in order to stabilize osmotically the resulting protoplasts (Fincham, 1989). The uptake of DNA by protoplasts is accomplished by incubating them with high-concentrated DNA and by the addition of up to 10 volumes of a 40 - 60\% PEG 4000 solution (Fincham, 1989). PEG has been observed to cause clumping and fusion of protoplasts, which is thought to facilitate the trapping of DNA to the fungal cells (Fincham, 1989). However, a current research suggested that PEG is unlikely to induce the coordination between DNA and the recipient cell surface, and the fusion of protoplasts is not the direct cause of DNA uptake; therefore, the role of PEG in DNA uptake still not described (Kuwano et al., 2008). Following the PEG treatment, protoplasts transferred to regeneration medium for recovery of the cell wall before being plated onto the selective medium. The selection of transformants can be achieved by using an auxotrophic complementation or drug resistance depending on the selectable marker gene built into the vector DNA.

Electroporation has been successfully used for the transformation of several fungi, including N. crassa, Aspergillus oryzae, Aspergillus niger, Rhizopus oryzae and Colletotrichum species (Dandan et al., 2017). By applying a high-voltage pulse that creates transitory small pores along the membrane surface, this procedure makes the cell membrane more permeable (Batista et al., 2016). Electroporation of protoplasts is the most widespread technique, although methods have also been developed for spores and mycelia (Dandan et al., 2017). The electroporation procedure for $M$. circinelloides protoplasts have been also described and successfully carried out (Gutiérrez et al., 2011). Biolistic transformation is also known as particle bombardment. In biolistic transformation, foreign DNA is adsorbed on the surface of tungsten or gold particles and further under the push of high pressure, the particles containing nucleic acid are injected into the host cell. Biolostic transformation has been utilized to successfully transform A. nidulans, Trichoderma reesei and also M. circinelloides (Gonzalez-Hernandez et al., 1997; Dandan et al., 2017). A. 
tumefaciens is a gram-negative plant pathogenic bacterium, naturally causes crown gall tumors in plants. A. tumefaciens is able to transfer part of its tumor-inducing plasmid (TDNA) into infected cells, where it integrates into the host genome. ATMT was implemented successfully to transform various fungal species, such as Fusarium circinatum, Agaricus bisporus, Calonectria morganii, Fusarium oxysporum, Verticillium fungicola, Monascus purpureus and M. circinelloides (Covert et al., 2001; Mikosch et al., 2001; Malonek and Meinhardt, 2001; Mullins et al., 2001; Amey et al., 2002; Campoy et al., 2003; Nyilasi et al., 2005; Papp et al., 2013).

In Mucoromycotina fungi artificial introduction of DNA by transformation is normally established and maintained by autonomously replicating plasmids (vanHeeswijck and Roncero, 1984; Garre et al., 2014). The first transformation of $M$. circinelloides was carried out by van-Heeswijck and Roncero (van-Heeswijck and Roncero, 1984). In this study 5'- upstream of the leuA gene (encoding the $\alpha$ isopropylmalate isomerase) an autonomously replicating site (ARS) was described (Roncero et al., 1989). The exact position and the sequence boundaries of this $M$. circinelloides ARS element remain unclear (Appel et al., 2004), however in $M$. circinelloides ARS element is not required to maintain the plasmids as extrachromosomal elements (van-Heeswijck and Roncero, 1984; Appel et al., 2004; Csernetics et al., 2011). Relatively high transformation frequency can be reached with circular plasmids, at the same time the copy number of plasmid often remains low, resulting in mitotic instability (Ibrahim and Skory, 2006). The integration happens rarely in Mucoromycotina fungi when using circular plasmids, moreover it also difficult to achieve with linear fragments (Papp et al., 2013). This can be due to a presumed mechanism, which can protect the fungal genome from foreign DNA (Ibrahim and Skory, 2006). Successful integration was achieved with ATMT in M. circinelloides; in these studies random integration was observed, but transformants found to be unstable (Nyilasi et al., 2005; Papp et al., 2013). Stable transformants were isolated when random integration was carried out with restriction enzyme-mediated integration via PEG-mediated transformation of $M$. circinelloides protoplasts (Papp et al., 2013). Recently, the CRISPR/Cas9 system has been used to target and disrupt genes in Aspergillus spp., T. reseii and S. cerevisiae (Fuller et al., 2015; Nødvig et al., 2015; Mans et al., 2015). Moreover, it was successfully adapted to $M$. circinelloides for guided integration of the foreign DNA into the genome. This genome editing tool seems to be the most efficient for creation of knock out $M$. circinelloides mutants (Nagy et al., 2017). 
Beside gene deletion, RNA-based gene silencing is also a frequently and successfully applied method for functional characterization of genes (Nicolás et al., 2003; Hood, 2004; Redberry, 2006). This approach seems to be beneficial when gene-targeting approaches fail, for example, when multiple copies of a gene of interest is present in the genome (Meyer, 2008). It is also a useful technique to study essential genes, when the deletion may be fatal to the organism (Liu et al., 2002; Kadotani et al., 2003). Three RNA-based (antisense RNA, hammerhead ribozymes and RNA interference) have been shown to be valuable tools for gene silencing in eukaryotes. All three silencing methods were already used successfully in various filamentous fungi (Moralejo et al., 2002; Kadotani et al., 2003). In eukaryotes, there are two mechanisms, each are related but distinct RNA silencing pathways (Nakayashiki, 2005). Based on the small RNA molecules involved, those are referred to as "small interfering RNA (siRNA)-directed pathway" and "microRNA (miRNA)-directed pathway". Both siRNAs and miRNAs interfere with gene expression via targeted degradation of mRNA or by the suppression of translation. The siRNAs act as guides for a siRNA-induced silencing complex to target perfectly complementary mRNAs for degradation. The second class of small RNAs, miRNAs is processed from imperfect stem-loop RNA precursors (pre-miRNAs) that are transcribed from non-protein-coding genes within plant and animal genomes. The mature miRNA causes the translational repression of the target mRNA that has imperfect complementarity to the miRNA (Nakayashiki, 2005).

In case of RNA interference, small RNA molecules direct the RNA-induced silencing complex that can degrade targeted mRNA molecules thereby blocking the translation into polypeptides (Schumann et al., 2010). All these small RNAs are derived from double stranded or hairpin RNA precursors createn by Dicer (Fig. 7) (Eamens et al., 2008; Li et al., 2010). RNA interference in fungi does not cause complete suppression of targeted gene (Weld et al., 2006; Schumann et al., 2010). While methods of gene knock down strategies require information of only small fragments of sequence, this can be implemented even in the organisms with less genetic information (Weld et al., 2006; Schumann et al., 2010). It has to be noted that efficiency of gene silencing method was not stable and constant in all fungi (Mouyna et al., 2004). 


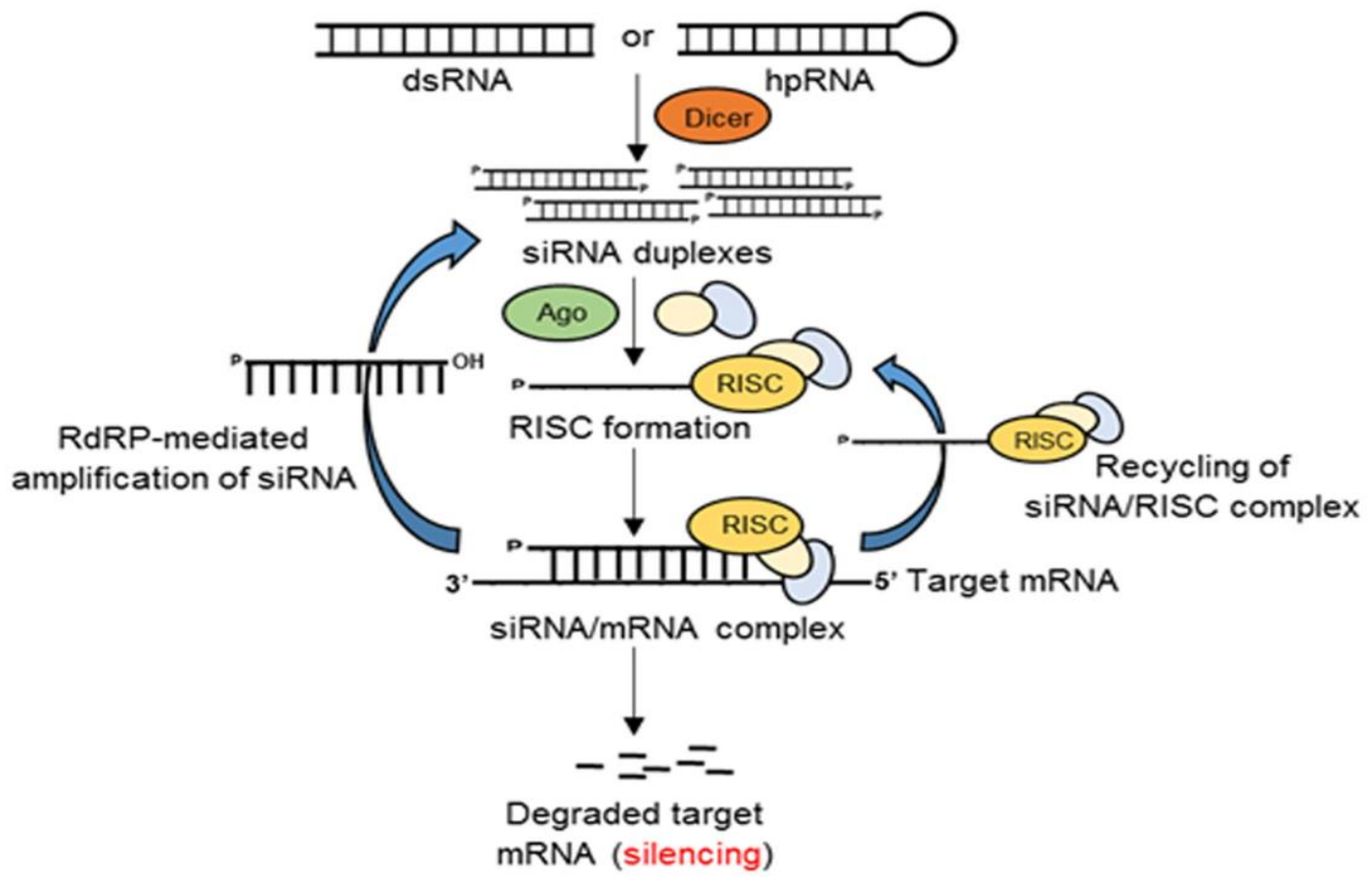

Fig 7. Schematic picture of RNA silencing mechanism in fungi (Majumdar et al., 2017).

Glyceraldehyde-3-phosphate dehydrogenase (GPD) is a catalyst in both glycolysis and gluconeogenesis. As a main housekeeping enzyme, its amino acid sequence demonstrates a strong structural conservation between different prokaryotic and eukaryotic organisms (Chapman et al., 2015). GPD is usually considered to be efficiently and constitutively expressed; for example, in $S$. cerevisiaes $2-5 \%$ of the poly (A) ${ }^{+}$RNA may constitute gpd mRNA (Ringel et al., 2013). On the basis of this feature, promoter of GPDencoding genes were found to be efficient for expression of heterologous genes in several yeasts and filamentous fungi, and have been used to construct efficient transformation systems in numerous fungal species at various taxonomic positions, e.g., Rhizomucor miehei, Paracoccidioides brasiliensis, A. nidulans and A. bisporus (Lima et al., 2003; Barbosa et al., 2004; Vastag et al., 2004; Lopes et al., 2008). The characterization of three individual genes ( $g p d 1$, gpd2, and $g p d 3$ ) encoding GPD together with the use of the promoter of the gpdl gene for recombinant protein production in M. circinelloides is well describe by Wolff and Arnau (Wolff and Arnau 2002). Only transcription of gpdl was detected and the transcription was found to be highly regulated in response to carbon source (Wolff and Arnau 2002; Larsen et al., 2004). The promoter of gpdl was used in several experiments to express of homologous or heterologous genes in M. circinelloides (Wolff and Arnau 2002; Larsen et al., 2004; Papp et al., 2006, 2013; Csernetics et al., 2011). 
A well-established $\mathrm{PEG} / \mathrm{CaCl}_{2}$-mediated transformation system is available for $M$. circinelloides, which is an often used as a model organism to study the carotenoid biosynthesis, dimorphism, sexual reproduction and pathogenicity (Gooday and Adams, 1993; Papp et al., 2006, 2013; Csernetics et al., 2011; Li et al., 2011; Wang and Lin, 2012; Lee et al., 2015; Ruiz-Vázquez et al., 2015; Zhang et al., 2016). Dominant selection markers are often not usable for Mucoromycotina fungi (Papp et al., 2010), thus it serves a great advantage that $M$. circinelloides MS12, a leucine and uracil auxotroph mutant $M$. circinelloides is available for the genetic modification (Benito et al., 1992). In addition, its genome sequence is available (Corrochano et al., 2016) making a good support for the characterization of genetic background of biological processes. 


\section{AIMS OF THE STUDY}

M. circinelloides (Mucoromycotina, Mucorales) is a carotenoid producing fungus, which is a model organism of several carotenogenic studies. Carotenoids together with other terpenoids, such as ergosterol or the prenyl groups of ubiquinones and the farnesylated and geranylgeranylated proteins, are synthesized via the mevalonateisoprenoid pathway in fungi. These metabolites play an important role in signal transduction, morphogenesis, adaptation to environmental change and protection against free radicals. Moreover, ergosterol and its biosynthesis are one of the main targets of the antifungal agents used to treat infections caused by Mucoromycotina fungi in clinics.

Till the date limited information is available about the function and regulation of the genes playing role in mevalonate-isoprenoid pathway in Mucoromycotina fungi. Characterization of these genes can serve valuable knowledge for the improvement of secondary metabolite production by Mucoromycotina fungi and may lead us to better understanding of biological processes, including morphogenesis, protection against free radicals, response to environmental change (e.g. changes in the soil composition, oxygen concentration or salinity of the environment) or pathogenesis.

In this study six genes involved in the mevalonate-isoprenoid biosynthesis in $M$. circinelloides, encoding the HMG-CoA synthase $(h m g S)$, mevalonate kinase $(m v k)$, diphosphomevalonate decarboxylase $(d m d)$, IPP isomerase (ipi), FPP and GGPP synthases (isoA and $\operatorname{car} G$ genes, respectively) were selected for characterization. One of our goals was to improve the carotenoid production of the fungus with overexpression and silencing of the genes. Our next aim was to examine the effect of the overexpression and silencing of the genes on the ergosterol content of $M$. circinelloides and it's effect on the susceptibility to different antifungals, which may lead to identify targets for new antifungal therapy. Little is known about the efficiency of gene silencing can be achieved with different plasmid constructs in $M$. circinelloides, thus among our aims was to compare the characteristics of the mutants harbouring different plasmids for gene knockdown as well.

The following specific objectives have been addressed to present study:

\section{Investigation of the transcription of selected six genes under different cultivation} conditions involved in the mevalonate-isoprenoid biosynthesis in M. circinelloides. 
Effect of cultivation conditions, such as temperature, oxygen tension, light sources, medium composition and incubation time on the transcription of the $h m g S, m v k$, $d m d$, ipi, isoA and carG genes.

2. Overexpression and silencing of selected six genes involved in the mevalonateisoprenoid biosynthesis in $M$. circinelloides and characterization of the transformants

Development of different plasmid constructs for overexpression and silencing of the $h m g S$, mvk, dmd, ipi, isoA and carG genes in $M$. circinelloides. Transformation experiments with the M. circinelloides MS12 strain. Characterization of the transformants in detail, such as analyzes of micromorphology, carotenoid and ergosterol content, antifungal susceptibility and interaction with macrophages. Comparison of the overexpression and gene silencing efficiency achieved with the different plasmid constructs. 


\section{MATERIALS AND METHODS}

\subsection{Strains used in the experiments}

During the transformation experiments the $M$. circinelloides $f$. lusitanicus leuA $A^{-}$and pyr $G^{-}$double auxotroph mutant $M$. circinelloides MS12 strain (SZMC 12082), derived from the CBS 277.49 isolate was used (Benito et al., 1992). Plasmid propagation was performed in Escherichia coli TOP $10 \mathrm{~F}^{-}$(Invitrogen, genotype: $\mathrm{F}^{\prime}\{$ lacIq, Tn10(TetR)\}

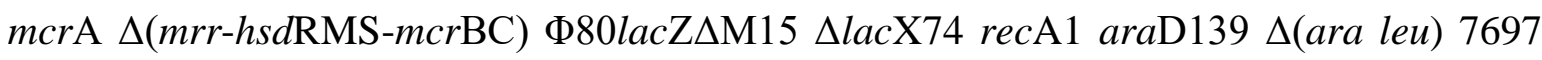
gal $\mathrm{U}$ galK $\operatorname{rps} \mathrm{L}(\mathrm{StrR})$ end $\mathrm{A} 1$ nup $\mathrm{G})$.

\subsection{Applied media and growth conditions}

\section{YNB minimal medium}

$1 \%$ D-glucose, $0.15 \%\left(\mathrm{NH}_{4}\right)_{2} \mathrm{SO}_{4}, 0.15 \%$ Na-L-glutamate, $0.05 \%$ YNB (yeast nitrogen base without amino acids, Sigma-Aldrich), supplemented with $0.05 \%$ uracil and/or $0.05 \%$ leucine $(\mathrm{w} / \mathrm{v})$ if required. For solid medium $2 \%(\mathrm{w} / \mathrm{v})$ agar was added. For $\mathrm{PEG} / \mathrm{CaCl}_{2}$-mediated protoplast transformation, YNB medium with $0.8 \mathrm{M}$ sorbitol containing $1 \%$ or $2 \%(\mathrm{w} / \mathrm{v})$ agar were used.

Occasionally composition of the YNB medium was modified according to the experiments: the glucose concentration was increased to $2.5 \%-5 \%(\mathrm{w} / \mathrm{v})$ or was replaced with dihydroxyacetone (DHA), sodium-acetate (Na-ac), maltose (Mal), or trehalose (Treh) or the medium was supplemented with $1-3 \%$ (w/v) $\mathrm{NaCl}$ or $0.5-4 \mu \mathrm{g} / \mathrm{ml} \mathrm{FLU.}$

\section{Yeast extract - Glucose medium (YEG)}

$1 \%$ D-glucose, $0.5 \%$ yeast extract, $2 \%$ agar (w/v) $(\mathrm{pH} 4.5)$.

\section{Yeast extract - Peptone - Glucose broth (YPG)}

$2 \%$ D-glucose, $1 \%$ peptone, $0.5 \%$ yeast extract $(\mathrm{w} / \mathrm{v})(\mathrm{pH} 4.5)$, supplemented with $0.8 \mathrm{M}$ sorbitol for regeneration of protoplasts.

\section{Luria-Bertani medium (LB)}

$1 \% \mathrm{NaCl}, 1 \%$ tryptone, $0.5 \%$ yeast extract (w/v) $(\mathrm{pH} 7.0)$, supplemented with $2 \%$ (w/v) agar for making solid medium. For the selection of transformant E. coli strains, the medium was supplemented with ampicillin in a final concentration of $100 \mu \mathrm{g} / \mathrm{ml}$. 


\section{Malt extract agar (MEA)}

$1 \%$ D-glucose, $0.5 \%$ yeast extract, $1 \%$ malt extract, $2 \%$ agar $(\mathrm{w} / \mathrm{v})$.

\section{Growth conditions and strain maintenance}

For cultivation of fungus $10^{6}$ spores were inoculated onto fresh media in $150 \mu 1$ final volume. Fungal strains were maintained on YNB medium (supplemented with uracil and/or leucine if necessary) at $4{ }^{\circ} \mathrm{C}$. Their cultivation was usually done at $25{ }^{\circ} \mathrm{C}$ for four days under constant illumination. Occasionally the inoculum size $\left(2 \times 10^{2}-10^{6}\right.$ spore/plate or flask), the temperature (between 15 and $37^{\circ} \mathrm{C}$ ), the light source (daylight (Sylvania Daylight F8W/T5/154), warm white (Osram Warm White L8W/32-930) light source and dark), oxygen tension (aerobic - anaerobic conditions) and growth time (from 4 hours to 4 days) was varied according to the experiments. For later use, the mycelium collected from the culture medium was stored frozen at $-20{ }^{\circ} \mathrm{C}$ (after distilled water wash).

When the effect of different cultivation conditions on the gene transcription was investigated the $M$. circinelloides MS12 strain was cultivated on solid YNB plates supplemented with leucine and uracil and grown for 4 days under continuous daylight illumination at $25{ }^{\circ} \mathrm{C}$. The inoculum size was $10^{6}$ sporangiospores/plate (control conditions). Occasionally, the cultivation was performed in $30 \mathrm{ml} \mathrm{YNB}$ liquid medium; the inoculum size was $10^{4}$ sporangiospores $/ \mathrm{ml}$. To investigation of the effect of illumination, the fungal strain was cultivated in dark, under continuous daylight or warm white light source or under continuous dark followed by $10 \mathrm{sec}$ of illumination and $10 \mathrm{~min}$ in dark. Daylight and warm white bulbs differing in the color temperature of the emitted light (3000 $\mathrm{K}$ and $6500 \mathrm{~K}$, respectively), the light color of a lamp depends on its spectral power distribution in the visible range; white light of the warm white source contains more components in the yellow-red wavelength range, while white light of the daylight source contains more components in the blue range. Temperature dependence of the gene transcription was tested by cultivating the fungal strain on solid YNB media at 15, 20, 25, 30 and $35^{\circ} \mathrm{C}$. To examine the effect of different carbon sources, glucose was replaced with Treh, Mal, DHA and Na-ac in a final concentration of $1 \%(\mathrm{w} / \mathrm{v})$ in YNB. To test the effect of the different salt and glucose concentrations, $\mathrm{NaCl}$ were added to solid $\mathrm{YNB}$ in a final concentration of 1, 2 and 3\%(w/v) or glucose of 1, 2.5 and $5 \%(\mathrm{w} / \mathrm{v})$, respectively. Our research group previously analyzed the susceptibility of $M$. circinelloides MS12 against different statins (such as fluvastatin (FLU), atorvastatin, rosuvastatin, simvastatin (SIM) and pravastatin) and FLU showed the most prominent inhibition effect on the fungal 
growth (unpublished data). To test the effect of FLU on the gene transcription, strains were grown in $30 \mathrm{ml}$ liquid YNB containing FLU in final concentrations of 0.5, 1, 2 and 4 $\mu \mathrm{g} / \mathrm{ml}$ for 4 days with shaking $(150 \mathrm{rpm})$ at $25^{\circ} \mathrm{C}$. Cultivation under anaerobic condition was performed in $30 \mathrm{ml}$ liquid $\mathrm{YNB}$ in a BBL GasPak Anaerobic System (Becton Dickinson) at $25^{\circ} \mathrm{C}$.

\subsection{Primers used in this study}

PCR primers were designed for the amplification of genes and gene fragments, sequencing and real-time PCR. The primers used in this study are shown in Table 2.

Table 2. Primers used in the experiments. Primer names, sequences, usage and the expected size of amplicons are also shown in the table. The recognition site of restriction endonucleases is shown in underlined and additional nucleotides upstream from the recognition sites are shown in normal letters

\begin{tabular}{|c|c|c|c|}
\hline Name of primer & Sequence 5'-3' & $\begin{array}{l}\text { Fragmet } \\
\text { size (bp) }\end{array}$ & Used for \\
\hline hmgS F & GCC TCA TCT AAA GCC TTC GT & \multirow[t]{2}{*}{3141} & \multirow{6}{*}{$\begin{array}{l}\text { amplification of the } M \text {. } \\
\text { circinelloides hmg } \text {, } \\
m v k \text { and dmd genes } \\
\text { together with their } \\
\text { regulatory regions }\end{array}$} \\
\hline hmgS R & AAA GAC CGG CTG GAA GAT GT & & \\
\hline mvk F & GCA ATG GTG ACC TCA ACC TCA A & \multirow[t]{2}{*}{2688} & \\
\hline mvk R & CGT AGG TCA AGA AAC CAT CCG A & & \\
\hline dmd F & CTA TGC CGT GCA ATG CGA GAT T & \multirow[t]{2}{*}{2679} & \\
\hline dmd R & GGA GGA GTG TGA AGC CTG TAA & & \\
\hline hmgS gpd F & gcgATCGAT ATG ACT GTC CCC AAC TAC AAC A & \multirow[t]{2}{*}{1560} & \multirow{6}{*}{$\begin{array}{l}\text { amplification of the } \\
\text { coding region of } M \text {. } \\
\text { circinelloides hmgS, } \\
m v k \text { and dmd genes }\end{array}$} \\
\hline hmgS gpd R & tatGCGGCCGC CTA ATC CTT ACG CTT GTA GAA A & & \\
\hline mvk gpd F & attGTCGAC ATG ACT GCA ACT GAA CAA A & \multirow[t]{2}{*}{1364} & \\
\hline mvk gpd R & cgcGAATTC TTA GTT TTG AAA GTA TTG TTC C & & \\
\hline dmd gpd F & cgcATCGAT ATG AAG ACT GTT ACC TGT ACT GCT CCC & \multirow[t]{2}{*}{1289} & \\
\hline dmd gpd R & taaGCGGCCGC CTA TGC AAG ACG CTT GGG AA & & \\
\hline hmgSas F & tcgATCGAT TGC GTT ATT CAG TCA GAG ATG & \multirow[t]{2}{*}{810} & \multirow{12}{*}{ 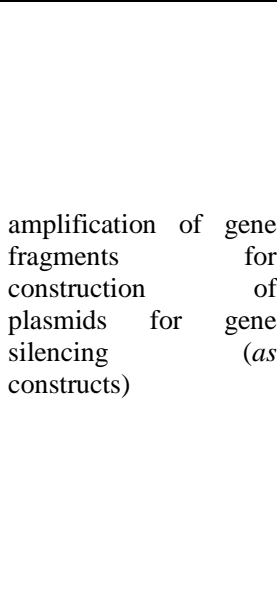 } \\
\hline hmgSas R & tatCTCGAG TGC ACG AGC AAA GGA CTT GTT & & \\
\hline mvkas F & tatGAGCTC CCC GTC ATC CTG ATG ATA TGC & \multirow[t]{2}{*}{800} & \\
\hline mvkas R & tcgATCGAT AAT AAA GGT AAC AGC GCA TCC & & \\
\hline dmdas F & tagATCGAT AGT GTC CAG AAT CGC TCG TCA & \multirow[t]{2}{*}{726} & \\
\hline dmdas R & gctCTCGAG TGG CTT AAT ACA CGA GGT CCA & & \\
\hline ipias F & gcgATCGAT GAT TTG AAG GAA TAT GAC GAA & \multirow[t]{2}{*}{887} & \\
\hline ipias R & tatCTCGAG GAT GAA TGG TAT CAT CGG CCT & & \\
\hline isoAas $\mathrm{F}$ & tcgATCGAT TGA TAT CAT GGA TGC TTC CAT C & \multirow[t]{2}{*}{843} & \\
\hline isoAas $\mathrm{R}$ & tagCTCGAG TTC TTG ACA CCG ACA GAT TCG T & & \\
\hline carGas F & ctgATCGAT GTG CCT GTC GCT CAC CAT ATT & \multirow[t]{2}{*}{858} & \\
\hline carGas R & aatCTCGAG TGG TGG CCT CTA CTC TGA TGG TC & & \\
\hline hmgS hpRNA1 F & tgtCTCGAG CCG TGC AAA ACT TGA TGG AAA A & \multirow[t]{2}{*}{691} & \multirow{5}{*}{$\begin{array}{l}\text { amplification of gene } \\
\text { fragments } \\
\text { construction }\end{array}$} \\
\hline hmgS hpRNA1 R & acgATCGAT GCC GGC CTA CAA TTG TGT GTT AGT CA & & \\
\hline hmgS hpRNA2 F & tacGCCGGC TGC ACG AGC AAA GGA CTT GTT & \multirow[t]{2}{*}{608} & \\
\hline hmgS hpRNA2 R & tcaGCGGCCGC CCG TGC AAA ACT TGA TGG AAA AG & & \\
\hline mvk hpRNA1 F & aggATCGAT GCC GGC GTA CCA TTT GAT CTA CAA AC & 829 & \\
\hline
\end{tabular}




\begin{tabular}{|c|c|c|c|}
\hline mvk hpRNA1 R & tcaGCGGCCGC TGA GAC CTG ATT GAG CAG TG & & \\
\hline mvk hpRNA2 F & aagCTCGAG TGA GAC CTG ATT GAG CAG TG & \multirow[t]{2}{*}{753} & \\
\hline mvk hpRNA2 R & gtaGCCGGC ATA CCC GTC ATC CTG ATG ATA T & & \\
\hline dmd hpRNA1 F & tatATCGAT GCC GGC GTA AGT TGG CTT GTC TGA TC & \multirow[t]{2}{*}{756} & \\
\hline dmd hpRNA1 R & tatGCGGCCGC TGT CCA AAA CAG CCT TCT TCA & & \\
\hline dmd hpRNA2 F & gtcCTCGAG TGT CCA AAA CAG CCT TCT TCA & \multirow[t]{2}{*}{700} & \\
\hline dmd hpRNA2 R & ataGCCGGC GTC ATC AAA TAT TGG GGC AA & & \\
\hline ipi hpRNA1 F & tcgCTCGAG ATG GCC CCT GAT TTG AAG GA & \multirow[t]{2}{*}{788} & \\
\hline ipi hpRNA1 R & cgtATCGAT GCC GGC CTA GCA TGT CAA TAA GGT CAG & & \\
\hline ipi hpRNA2 F & agtGCCGGC TGC TGT CAG CAA ACA TGG CCT & \multirow[t]{2}{*}{731} & \\
\hline ipi hpRNA2 R & tatGCGGCCGC ATG GCC CCT GAT TTG AAG GA & & \\
\hline isoA hpRNA1 F & ttcATCGAT GCC GGC GTA AGT TGT TCT TAT CAC TGG & \multirow[t]{2}{*}{753} & \\
\hline isoA hpRNA1 $\mathrm{R}$ & agtGCGGCCGC GTC AAG GAT CTT GCG TTG TTC G & & \\
\hline isoA hpRNA2 F & 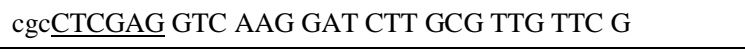 & \multirow[t]{2}{*}{693} & \\
\hline isoA hpRNA2 R & taaGCCGGC CTT CAA GCC TTC TTT TTG GT & & \\
\hline carG hpRNA1 F & tcgCTCGAG ATG CTC AAC TCA CAC AAC AGA & \multirow[t]{2}{*}{656} & \\
\hline carG hpRNA1 R & tatATCGAT GCC GGC CTG CCC GAT CGT GTT ACT C & & \\
\hline carG hpRNA2 F & catGCCGGC TGT TGT TGA CCA TAT CAA TGT ACT C & \multirow[t]{2}{*}{601} & \\
\hline carG hpRNA2 R & tatGCGGCCGC ATG CTC AAC TCA CAC AAC AGA & & \\
\hline hmgSRT F & TCC AAC ACT TGC TAC ATT CGT & \multirow[t]{2}{*}{112} & \multirow{14}{*}{$\begin{array}{l}\text { quantitative real-time } \\
\text { PCR }\end{array}$} \\
\hline hmgSRT R & GGC AGA CAA CAT AAT CAA TAT CGT C & & \\
\hline mvkRT F & GAT CGC TAT TCG TTG TCG TG & \multirow[t]{2}{*}{122} & \\
\hline mvkRT R & CAA GTC CAT CGA GCA AAC AG & & \\
\hline dmdRT F & CGT GAT TCC AAC CAG TTC CA & \multirow[t]{2}{*}{139} & \\
\hline dmdRT R & AAG CAG CCT TCA ATT TAC CGT & & \\
\hline ipiRT F & ATG TGG ACC AAC ACT TGC TGC TC & \multirow[t]{2}{*}{176} & \\
\hline ipiRT R & TTG ATG CCA AGC TCA TGC TCC AG & & \\
\hline isoART F & ATC TCG ACT GTT ACG GTG CTC CT & \multirow[t]{2}{*}{119} & \\
\hline isoART R & CTT GCG TTG TTC GGG ATT AGC CA & & \\
\hline carGRT F & CAA CAT CAT CAG CCA GAA GCC CA & \multirow[t]{2}{*}{148} & \\
\hline carGRT R & ACC ACC CAA ACG CTT GAT TTC CT & & \\
\hline actART F & CAC TCC TTC ACT ACC ACC GCT GA & \multirow[t]{2}{*}{117} & \\
\hline actART R & GAG AGC AGA GGA TTG AGC AGC AG & & \\
\hline gpdP & CAT GAA GTG TGA GAC ATT GCG A & \multirow{10}{*}{-- } & \multirow{10}{*}{$\begin{array}{l}\text { investigation of the } \\
\text { presence of plasmids in } \\
\text { transformants }\end{array}$} \\
\hline gpdT & TAC ATA TCA GAG GGT TGG AAC A & & \\
\hline ipigpd F & ggCTCGAG ATG GCC CCT GAT TTG AAG GAA TA & & \\
\hline ipigpd $\mathrm{R}$ & ctcGCGGCCGC TTA GAA GCC TAA ACG ATG AAT GGT & & \\
\hline isoAgpd $\mathrm{F}$ & ggCTCGAG ATG GTT GCT GTC AAA TTA CA & & \\
\hline isoAgpd R & ctcGCGGCCGC TTA TTT AGT ACG CTT GTA AA & & \\
\hline carGgpd F & ggCTCGAG ATG CTC AAC TCA CAC AAC AG & & \\
\hline carGgpd R & ctcGCGGCCGC CTA GTC GTT GGT GGC CTC TA & & \\
\hline amprev1 & GGC GAC ACG GAA ATG TTG AAT AC & & \\
\hline amprev2 & CGA AAT AGA CAG ATC GCT GAG & & \\
\hline
\end{tabular}




\subsection{Plasmid constructs used in this study}

M. circinelloides MS12 strain is a leucine and uracil double auxotroph mutant. The pAVB107 and pEPM901 (pEPM9) plasmids harbour the leuA and pyrG genes (encoding the $\alpha$-isopropylmalate isomerase and orotidine 5'-monophosphate decarboxylase, respectively), which complement the leucine and uracil auxotrophy, were constructed previously (Roncero et al., 1989; Benito et al., 1992; Velayos 2000; Csernetics et al., 2011). The plasmid pPT81, which harbours the $M$. circinelloides gpdl (encoding the glyceraldehyde-3-phosphate dehydrogenase 1, EMBL Acc. No.: AJ293012) promoter and terminator regions ( $g p d P$ and $g p d T$ ) and the $p y r G$ gene (in a previous study named as pPT43pyr, Csernetics et al., 2011; Nagy et al., 2014), and pMAT1811, provided by Victoriano Garré (Universidad de Murcia, Murcia, Spain), which harbours the gpdl and zrt1 (encoding a ZIP zinc transporter protein, NCBI Acc. No.: MUCCIDRAFT_155215) promoter regions $(z r t 1 P)$ facing to each other and the $p y r G$ gene (unpublished), was also used in cloning experiments (Wolff and Arnau, 2002; Csernetics et al., 2011; Trieu et al., 2017) (Suppl. 1).

Different plasmids were constructed for overexpression and silencing of the six genes. For overexpression the genes were amplified together with their own promoter and terminator regions (1) or were placed under the control of the M. circinelloides gpdl promoter and terminator (2) (own and gpd plasmid constructs, respectively; Fig. $8 \mathrm{~A}$ and B):

(1) The $h m g S, m v k$ and $d m d$ genes (Suppl. 2, unpublished) were amplified together with their regulatory regions with the hmgS F $-\mathrm{hmgS} \mathrm{R}$, mvk F - mvk R and dmd F-dmd $\mathrm{R}$ primers (Table 2), respectively, and were ligated into pBluescript SK+ (Stratagene) or pJET1.2/blunt cloning vectors. The plasmids were digested with NaeI - ClaI, Not I - Pst I and Not I - XhoI restriction endonucleases, and the fragments harbouring the hmgS, $m v k$ and dmd genes were inserted to the corresponding sites of the pEPM901 plasmid. Thus the constructed plasmids (pHMGS/own, pMVK/own and pDMD/own) harbour the hmgS, mvk or $d m d$ genes together with their own regulatory regions and the $p y r G$ marker gene (Table 3, Fig. 8 A). Plasmids, carry the ipi (NCBI Acc. No.: AM903092), isoA (NCBI Acc. No.: AJ496299) and carG (NCBI Acc. No.: AJ276129) genes with their own regulatory regions and leuA or pyrG marker genes - named pIPI/own, pISOA/own and pCARG/own - had been constructed previously (in that study named as pCA10, pAVB160 and pCA6, respectively, Csernetics et al., 2011) (Table 3, Fig. 8 A). 
(2) The coding regions of $h m g S, m v k$ and $d m d$ genes were amplified with the hmgS gpd F - hmgS gpd R, mvk gpd F - mvk gpd R and dmd gpd F - dmd gpd R primer pairs (Table 2) and the PCR products were ligated into pJET1.2/blunt cloning vector. The hmgS and $d m d$ were digested from the plasmids with $C l a \mathrm{I}-$ Not I restriction endonucleases and ligated into the corresponding sites of pPT81 plasmid, between the gpdl promoter and terminator regions. The $m v k$ gene was digested from the plasmid with $S a l I-E c o R I$ and was ligated into the gpdl promoter and terminator of pPT43 plasmid and then the fragment digested with NaeI - SacI was ligated into pEPM901. The constructed pHMGS/gpd, $\mathrm{pMVK} / \mathrm{gpd}$ and $\mathrm{pDMD/gpd}$ plasmids harbour the $h m g S, m v k$ or $d m d$ genes within the gpdl promoter and terminator regions and the $p y r G$ marker gene (Table 3, Fig. 8 B). Plasmid, carry the ipi, isoA and carG genes under the control of the gpdl regulatory regions and pyrG or leuA marker genes - named pIPI/gpd, pISOA/gpd and pCARG/gpd - were constructed previously (in that study named as pPT82, pPT86 and pPT87, respectively, Csernetics et al. 2011 and unpublished data) (Table 3, Fig. 8 B).

For gene silencing three different plasmids were constructed for all six genes: (1) the genes were inserted between the Mucor gpdl and zrtl promoter (Fig. 8 C); (2) a fragment of a gene was inserted between the Mucor gpdl promoter and terminator in inverted orientation (Fig. 8 D); (3) a fragment of a gene and its reverse complement together with an intron were placed under the regulation of the gpdl promoter and terminator (Fig. $8 \mathbf{E}$ ) (pMAT, as and hpRNA plasmid constructs, respectively):

(1) The pMAT1811 plasmid harbours the gpdl and zrtl promoter regions facing to each other and the pyrG marker gene (unpublished). The hmgS, mvk, dmd, ipi, isoA and $\operatorname{carG}$ genes were digested from pHMGS/gpd (XhoI - NotI, with reduced XhoI enzyme and digestion time), pMVK/gpd (SalI - EcoRI), pDMD/gpd (ClaI - NotI), pIPI/gpd (XhoI NotI), pISOA/gpd (XhoI - NotI) and pCARG/gpd (XhoI - NotI) plasmids, respectively, and were placed between the corresponding sites of pMAT1811, inside the gpdl and zrt promoters, resulted in the pHGMS/pMAT, pMVK/pMAT, pDMD/pMAT, $\mathrm{pIPI/pMAT,}$ pISOA/pMAT and pCARG/pMAT plasmids (Table 3, Fig. 8 C).

(2) The amplified fragments with hmgSas F-hmgSas R, dmdas F-dmdas R, ipias $\mathrm{F}$ - ipias R, isoAas $\mathrm{F}$ - isoAas $\mathrm{R}$, carGas $\mathrm{F}$ - carGas R primer pairs (Table 2) were ligated into pJET1.2/blunt cloning vector. The fragments were cut off with $C l a \mathrm{I}-X h o \mathrm{I}$ restriction endonucleases and were placed into the corresponding sites of pPT81. In case of $m v k$ PCR amplification was unsuccessfull, thus from pMVK/gpd a fragment digested with ClaI NotI was ligated into the corresponding sites of pPT81. The resulted plasmids - 
pHMGS/as, pMVK/as, pDMD/as, pIPI/as, pISOA/as, pCARG/as - harbours a fragment of the mevalonate-isoprenoid pathway genes between the gpdl promoter and terminator in inverted orientation and the $p y r G$ marker gene (Table 3, Fig. 8 D).
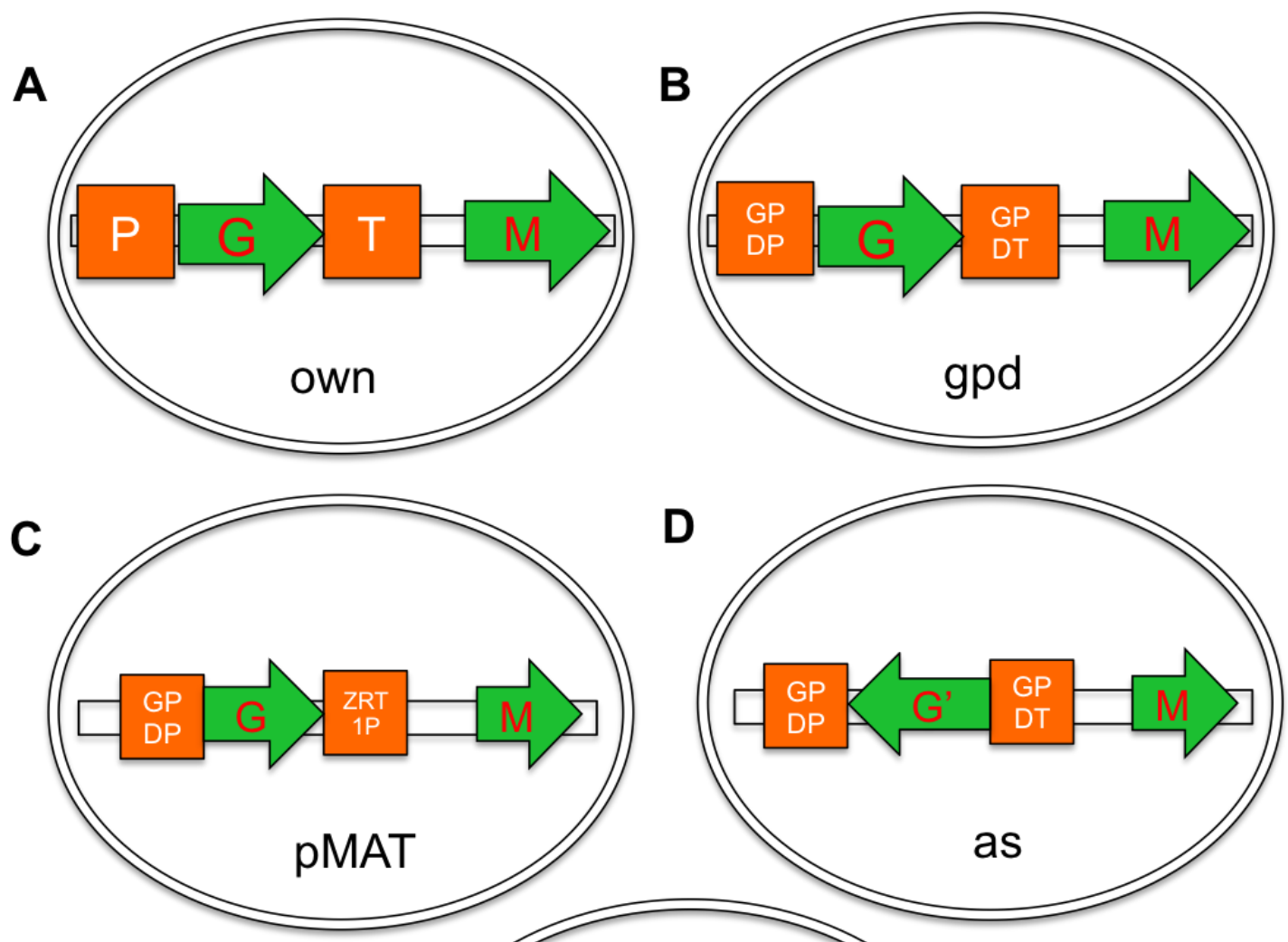

E

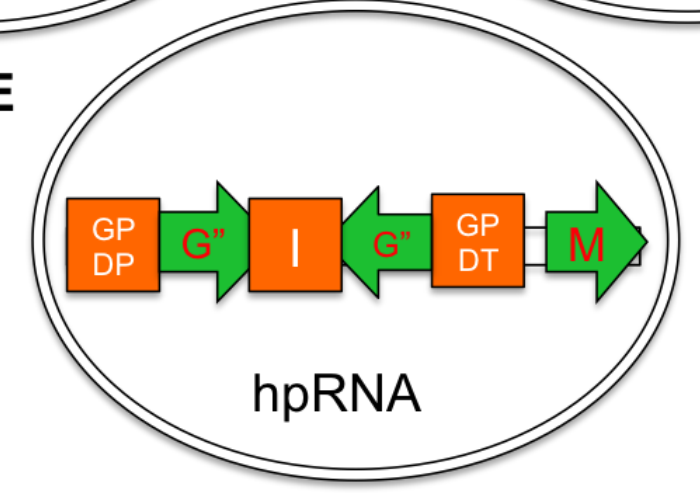

Fig 8. Schematic picture of plasmid constructions used in this study. The plasmid own and gpd were constructed for overexpression of the genes, while plasmids $p M A T$, as and $h p R N A$ were constructed for gene silencing. The genes were amplified with their own promoter and terminator (A), or were placed under the control of $M$. circinelloides gpdl regulatory regions (B) for overexpression. For gene silencing the genes were inserted between the Mucor gpdl and zrtl promoter (C), a fragment of genes were inserted between the $M$. ciricinelloides gpdl promoter and terminator in inverted orientation (D), and a fragment of a gene and its reverse complement together with an intron were placed under the regulation of the gpdl promoter and terminator (E). M - marker gene (Mucor pyrG or leuA) used for selection of the transformants. 
(3) To the hpRNA-mediated gene silencing two fragments were amplified for each gene with primers shown in Table 2: a fragment of a gene together with an intron downstream and its reverse complement without the intron. The fragments were ligated into pTZ57R/T or pJET1.2/blunt cloning vectors. The fragments together with the intron were digested from plasmids with ClaI - XhoI or ClaI - NotI restriction endonucleases and ligated into the corresponding sites of pPT81, between the gpdl promoter and terminator. Next, the fragments without the introns have been digested from the plasmids with Not I NaeI or XhoI - NaeI restriction endonucleases and ligated into the corresponding sites of pPT81. The resulted plasmids - pHMGS/hpRNA, pMVK/hpRNA, pDMD/hpRNA, $\mathrm{pIPI/hpRNA,} \mathrm{pISOA/hpRNA} \mathrm{and} \mathrm{pCARG/hpRNA} \mathrm{-} \mathrm{carry} \mathrm{a} \mathrm{fragment} \mathrm{of} \mathrm{a} \mathrm{gene} \mathrm{and} \mathrm{its}$ reverse complement together with an intron under the regulation of the gpdl promoter and terminator and the pyrG marker gene (Table 3, Fig. 8 E).

\subsection{Experimental methods}

\subsubsection{Extraction of genomic DNA from M. circinelloides}

For DNA purification GeneElute ${ }^{\mathrm{TM}}$ Plant Genomic DNA Miniprep Kit (SigmaAldrich) was used following the instructions of the manufacturer. Occasionally, the total DNA was purified with home-made solutions. In that case mycelium of $M$. circinelloides was disrupted in liquid nitrogen with mortar and pestle and $2.5 \mathrm{ml}$ lyses buffer $(50 \mathrm{mM}$ Tris (pH 8.0), 20 mM EDTA, 1\% sodium lauroyl sarcosinate (w/v)) was added for each gram of mycelium powder. After mixing with $5 \mu \mathrm{l}$ of $20 \mu \mathrm{g} / \mathrm{ml}$ RNase A (Sigma) the mixture was incubated at $65{ }^{\circ} \mathrm{C}$ for $30 \mathrm{~min}$. After cooling down to room temperature, the mixture was centrifuged $\left(8,000 \times \mathrm{g}, \quad 10 \mathrm{~min}, \quad 4{ }^{\circ} \mathrm{C}\right)$ and an equal volume of PCI (phenol:chloroform:isoamyl alcohol mixed in 25:24:1 ratio (v/v)) was added to the supernatant. The mixture was gently shaken $(45 \mathrm{rpm})$ for $2-3$ hours at $4{ }^{\circ} \mathrm{C}$. After centrifugation $\left(8,000 \times \mathrm{g}, 10 \mathrm{~min}, 4{ }^{\circ} \mathrm{C}\right)$ the aqeous phase was washed with $\mathrm{CI}$ (chloroform:isoamyl alcohol mixed in 24:1 ratio $(\mathrm{v} / \mathrm{v})$ ). The aqeous phase was collected in a fresh tube and the DNA was precipitated with $96 \%$ (v/v) ethanol at $-20{ }^{\circ} \mathrm{C}$ overnight. After centrifugation $(13,000 \times \mathrm{g}, 20 \mathrm{~min}$, room temperature) the DNA was washed with $70 \%(\mathrm{v} / \mathrm{v})$ ethanol and was dried under vacuum. The precipitate was dissolved in $500 \mu \mathrm{l}$ nuclease-free distilled water. 
Table 3. Plasmids used in this study and their characteristics.

\begin{tabular}{|c|c|c|c|c|}
\hline Plasmid & Gene cassette & Experiment & $\begin{array}{l}\text { Marker } \\
\text { gene }\end{array}$ & Reference \\
\hline pAVB107 & - & Control & leuA & Velayos et al. (2000) \\
\hline pEPM901 & - & Control & pyrG & Benito et al. (1992) \\
\hline pPT81 (pPT43pyr) & $\begin{array}{l}\text { gpdl promoter and } \\
\text { terminator }\end{array}$ & Vector constructions & $\operatorname{pyr} G$ & Csernetics et al. (2011) \\
\hline pPT43 & $\begin{array}{c}\text { gpdl } \\
\text { promoter and terminator }\end{array}$ & Vector constructions & - & Csernetics et al. (2011) \\
\hline pHMGS/own & \multirow{6}{*}{$\begin{array}{l}\text { Genes amplified with } \\
\text { own promoter and } \\
\text { terminator }\end{array}$} & \multirow{6}{*}{ Overexpression } & pyrG & This study \\
\hline pMVK/own & & & pyrG & This study \\
\hline pDMD/own & & & pyrG & This study \\
\hline pIPI/own & & & pyrG & Csernetics et al. (2011) \\
\hline pISOA/own & & & leuA & Csernetics et al. (2011) \\
\hline pCARG/own & & & pyrG & Csernetics et al. (2011) \\
\hline pHMGS/gpd & \multirow{6}{*}{$\begin{array}{l}\text { Genes under the control } \\
\text { of gpdl promoter and } \\
\text { terminator }\end{array}$} & \multirow{6}{*}{ Overexpression } & pyrG & This study \\
\hline pMVK/gpd & & & pyrG & This study \\
\hline pDMD/gpd & & & pyrG & This study \\
\hline $\mathrm{pIPI/gpd}$ & & & pyrG & Csernetics et al. (2011) \\
\hline pISOA/gpd & & & leuA & Csernetics et al. (2011) \\
\hline pCARG/gpd & & & leuA & Csernetics et al. (2011) \\
\hline pHMGS/MAT & \multirow{6}{*}{$\begin{array}{l}\text { Fragment of gene under } \\
\text { the control of } g p d l \text { and } \\
z r t 1 \text { promoter facing to } \\
\text { each other }\end{array}$} & \multirow{6}{*}{ Gene silencing } & \multirow{6}{*}{$\operatorname{pyr} G$} & \multirow{6}{*}{ This study } \\
\hline pMVK/MAT & & & & \\
\hline pDMD/MAT & & & & \\
\hline pIPI/MAT & & & & \\
\hline pISOA/MAT & & & & \\
\hline pCARG/MAT & & & & \\
\hline pHMGS/as & \multirow{6}{*}{$\begin{array}{l}\text { Fragment of gene under } \\
\text { control of } g p d 1 \\
\text { promoter and terminator } \\
\text { in inverted orientation }\end{array}$} & \multirow{6}{*}{ Gene silencing } & \multirow{6}{*}{$\operatorname{pyr} G$} & \multirow{6}{*}{ This study } \\
\hline $\mathrm{pMVK} / \mathrm{as}$ & & & & \\
\hline $\mathrm{pDMD} / \mathrm{as}$ & & & & \\
\hline $\mathrm{pIPI} / \mathrm{as}$ & & & & \\
\hline pISOA/as & & & & \\
\hline pCARG/as & & & & \\
\hline pHMGS/hpRNA & \multirow{6}{*}{$\begin{array}{l}\text { Fragment of gene and } \\
\text { its reverse complement } \\
\text { together with an intron } \\
\text { under the regulation of } \\
\text { the } g p d l \text { promoter and } \\
\text { terminator }\end{array}$} & \multirow{6}{*}{ Gene silencing } & \multirow{6}{*}{$\operatorname{pyr} G$} & \multirow{6}{*}{ This study } \\
\hline pMVK/hpRNA & & & & \\
\hline pDMD/hpRNA & & & & \\
\hline pIPI/hpRNA & & & & \\
\hline pISOA/hpRNA & & & & \\
\hline pCARG/hpRNA & & & & \\
\hline
\end{tabular}

\subsubsection{Agarose gel electrophoresis and isolation of DNA from agarose gel}

For agarose gel electrophoresis $0.8-2 \%(\mathrm{w} / \mathrm{v})$ agarose (depending on the size of the expected fragments) was dissolved in TAE buffer (40 mM Tris-acetic acid (pH 7.6), 1 $\mathrm{mM} \mathrm{Na} 2 \mathrm{EDTA}$ ). The separation was performed in TAE buffer with $80-110 \mathrm{~V}$ for $1-4$ hour. For visualization of nucleic acid under UV-light $0.5 \mu \mathrm{g} / \mathrm{ml}$ ethidium bromide (from $10 \mathrm{mg} / \mathrm{ml}$ stock solution, dissolved in distilled water, Sigma) was used in the experiments. 
The sample buffer was $1 \times$ DNA Loading Dye (in final concentration, Thermo Scientific), to determine the size of the bands GeneRuler $1 \mathrm{~kb}$ DNA Ladder (Thermo Scientific) was applied.

The DNA was cut off from the agarose gel with sterile scalp under UV light and DNA was obtained using the GenElute Minus EtBr Spin Column Kit (Sigma-Aldrich) or Zymoclean Large fragment DNA Recovery Kit (Zymo Research) according to the manufacturer's instructions.

\subsubsection{Applied PCR techniques and reaction conditions}

For cloning PCR reactions were carried out in a T3 Thermocycler (Biometra). The primers were synthesized with Integrated DNA Technologies (IDT and Bio-ScienceHungary). The applied primers are shown in Table 2.

To amplify genes or gene fragments from genomic DNA Phusion High Fidelity DNA Polymerase (Thermo Scientific) was used according to the manufacturer's recommendations. The reactions were prepared in a final volume of $25 \mu \mathrm{l}$ as follows:

50 - 100 ng genomic DNA

$0.4-0.4 \mu \mathrm{M}$ specific primer

$0.2 \mathrm{mM}$ dNTP mix (Thermo Scientific)

$1 \times$ Phusion $H F$ or $G C$ buffer

0.6 U Phusion High Fidelity DNA Polymerase

Amplification conditions were as follows: $98{ }^{\circ} \mathrm{C} 3 \mathrm{~min}$, [35 cycles: $98{ }^{\circ} \mathrm{C} 30 \mathrm{sec}$, $\left.58-72{ }^{\circ} \mathrm{C} 30 \mathrm{sec}, 72{ }^{\circ} \mathrm{C} 10-100 \mathrm{sec}\right]$ and $72{ }^{\circ} \mathrm{C} 10 \mathrm{~min}$, cooling to $4{ }^{\circ} \mathrm{C}$. The annealing temperature and extension time was modified according to the primers and length of the expected amplicons.

\subsubsection{Restriction digestion and ligation}

Restriction digestions and ligations were performed according to the commonly used methods and following the manufacturer's instructions with optimization to the particular experimental conditions (Sambrook et al., 1989). DNA fragments were ligated into pJET1.2/blunt (CloneJET PCR Cloning Kit, Thermo Scientific) and pTZ57R/T (Thermo Scientific) cloning vectors following the manuals. 


\subsubsection{Determination of sequences}

Sequencing of positive clones was performed with LGC Genomics (Berlin, Germany). Sequences were analyzed with BioEdit Sequence Alignment Editor, NCBIBLAST (National Center for Biotechnology Information - Basic Local Alignment Search Tool) (http://blast.ncbi.nlm.nih.gov/Blast.cgi) and Expasy Bioinformatics Resource Portal (http://www.expasy.ch).

\subsubsection{Preparation and transformation of competent $E$. coli cells and plasmid DNA purification}

$200 \mu \mathrm{l}$ of $E$. coli $\mathrm{TOP} 10 \mathrm{~F}^{-}$suspension was inoculated into LB broth from glycerol stock and were incubated for 16 hours at $37^{\circ} \mathrm{C}$. After that, fresh $30 \mathrm{ml} \mathrm{LB}$ medium was inoculated with $1 \mathrm{ml}$ of the 16 hours cultures and the $E$. coli was grown with shaking at 200 $\mathrm{rpm}$ at $37^{\circ} \mathrm{C}$ up to $\mathrm{OD}_{660}=0.6$. The culture was centrifuged $\left(2160 \times \mathrm{g}, 10 \mathrm{~min}, 4{ }^{\circ} \mathrm{C}\right)$ and then the cells were washed with $15 \mathrm{ml} 100 \mathrm{mM}$ ice-cold $\mathrm{CaCl}_{2}$ solution. After centrifugation $\left(2160 \times \mathrm{g}, 10 \mathrm{~min}, 4^{\circ} \mathrm{C}\right)$, the cells were resuspended in $30 \mathrm{ml} 100 \mathrm{mM}$ icecold $\mathrm{CaCl}_{2}$ solution and incubated for 1 hour at $4{ }^{\circ} \mathrm{C}$. After centrifugation $(2160 \times \mathrm{g}, 10$ min, $4{ }^{\circ} \mathrm{C}$ ) and washing with $100 \mathrm{mM}$ ice-cold $\mathrm{CaCl}_{2}$ the sedimented cells were resuspended in 1/20 volume of cold, $20 \%$ (v/v) glycerol containing $100 \mathrm{mM} \mathrm{CaCl}$. Competent cells were dispensed into $100 \mu \mathrm{l}$ aliquots and stored at $-70{ }^{\circ} \mathrm{C}$ until later use.

For $E$. coli transformation $100 \mu \mathrm{l}$ of frozen competent cells were thawed on ice and $20 \mu \mathrm{l}$ of ligation mixture and $80 \mu \mathrm{TCM}$ buffer $(10 \mathrm{mM}$ Tris $(\mathrm{pH} 7.5), 10 \mathrm{mM} \mathrm{CaCl} 2,10$ $\mathrm{mM} \mathrm{MgCl} 2$ ) were added to the cells. The mixture was incubated for $30 \mathrm{~min}$ on ice. Heat shock was performed on $37{ }^{\circ} \mathrm{C}$ for 3.5 min and subsequently the mixture was incubated for $10 \mathrm{~min}$ at room temperature. E. coli cells were finally plated onto $100 \mu \mathrm{g} / \mathrm{ml}$ ampicillin containing LB medium $(50 \mathrm{mg} / \mathrm{ml}$ ampicillin sodium salt stock solution dissolved in distilled water, Sigma). For blue-white screening of positive clones $20-20 \mu 1$ of X-Gal (20 $\mathrm{mg} / \mathrm{ml}$ stock solution dissolved in dimethylformamide, Thermo Scientific) and IPTG (isopropyl- $\beta$-D-thiogalactopyranoside, $20 \mathrm{mg} / \mathrm{ml}$ stock solution dissolved in water, Thermo Scientific) were added to the media. Petri dishes were incubated for 16 hours at $37^{\circ} \mathrm{C}$.

For plasmid DNA purification from E. coli Mini Plus Plasmid DNA Extraction System (Viogene) and Midi Plus Ultrapure Plasmid DNA Extraction System (Viogene) were used following the instructions of the manufacturer. 


\subsubsection{Total RNA purification from $M$. circinelloides and cDNA synthesis}

For RNA extraction E.Z.N.A. ${ }^{\circledR}$ Fungal RNA Kit (Omega Bio-tek) and E.Z.N.A. ${ }^{\circledR}$ Total RNA Kit II (Omega Bio-tek) were used following the instructions of the manufacturer. RNA samples were treated with DNase I (Thermo Scientific) as follows:

$3 \mu \mathrm{g}$ RNA

$1 \times$ reaction buffer with $\mathrm{MgCl}_{2}$

3 U DNase

the final volume was set with diethyl pyrocarbonate-treated water.

The reaction was carried out at $37^{\circ} \mathrm{C}$ for $30 \mathrm{~min}$ and then quenched with $3 \mu 150$ mM EDTA at $65{ }^{\circ} \mathrm{C}$ for $10 \mathrm{~min}$. Each sample was checked with PCR or qPCR for DNA contamination. Reverse transcription was performed with RevertAid H Minus First Strand cDNA Synthesis Kit (Thermo Scientific) or with the Maxima H Minus First Strand cDNA Synthesis Kit (Thermo Scientific). For the reaction, oligo (dT) 18 and random hexamer primers were used according to the manufacturer's instructions.

\subsubsection{Quantitative real-time PCR}

The qPCR reactions were prepared using Maxima SYBR Green qPCR Master Mix (2×) (Thermo Scientific) and was set on 96 well plates with $20 \mu \mathrm{l}$ final volume as follows:

200 - 500 ng DNA or cDNA

$0.4 \mu \mathrm{M}-0.4 \mu \mathrm{M}$ specific primer (Table 2)

$1 \times$ Maxima SYBR Green qPCR Master Mix

The reactions were performed with C1000 Thermal Cycler with CFX96 Real-Time System (Bio-Rad) detecting system. The results were evaluated using the $2^{-\Delta \Delta \mathrm{Ct}}$ method (Livak and Schmittgen, 2001).

Amplification conditions were as follows: $95{ }^{\circ} \mathrm{C} 3 \mathrm{~min},\left[40\right.$ cycle: $95{ }^{\circ} \mathrm{C} 15 \mathrm{sec}, 60$ ${ }^{\circ} \mathrm{C} 30 \mathrm{sec}, 72{ }^{\circ} \mathrm{C} 30 \mathrm{sec}$. Melting curve analyzis was performed between $65^{\circ} \mathrm{C}-95^{\circ} \mathrm{C}$ with a $0.5^{\circ} \mathrm{C}$ increment. 


\subsubsection{Protoplast formation and $\mathrm{PEG} / \mathrm{CaCl}_{2}$-mediated transformation of $M$. circinelloides}

For protoplast formation Mucor spores harvested from cultures grown for 4 days, were inoculated in small drops onto cellophane sheets placed on YEG or YNB medium supplemented with uracil and leucine, and grown at $25^{\circ} \mathrm{C}$ for 16 hours (Nagy et al., 1994; Papp et al., 2006; Csernetics et al., 2011). Young colonies were transferred into protoplast forming solution (10 mM sodium-phosphate buffer, $\mathrm{pH} 6.4 ; 0.8 \mathrm{M}$ sorbitol; $1.5 \%(\mathrm{w} / \mathrm{v})$ home-made snail gut enzyme; $100 \mathrm{mM}$ sodium phosphate buffer was prepared with mixing of $25 \mathrm{mM} \mathrm{Na} 2 \mathrm{HPO}_{4}$ and $75 \mathrm{mM} \mathrm{NaH}_{2} \mathrm{PO}_{4}$ ) and incubated at $25{ }^{\circ} \mathrm{C}$ for 3 hours with continuous gentle shaking $(60 \mathrm{rpm})$. Protoplasts were separated from mycelia with filtration through three sheets of gauze, collected with centrifugation $\left(2162 \times \mathrm{g}, 15 \mathrm{~min}, 4{ }^{\circ} \mathrm{C}\right)$ and washed once with $5 \mathrm{ml}$ sorbitol - MOPS - calcium chloride buffer (SMC: $0.8 \mathrm{M}$ sorbitol;

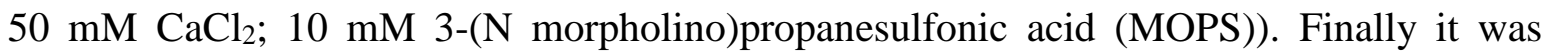
resuspended in $250 \mu 1 \mathrm{SMC}$ buffer.

The modified PEG-mediated transformation described by van-Heeswijck and Roncero was applied for transformation of protoplasts (van-Heeswijck and Roncero, 1984; Csernetics et al., 2011). To the resuspended protoplasts 5-10 $\mu \mathrm{g}$ of purified plasmid DNA was added together with $20 \mu$ of PEG - sorbitol - MOPS - calcium chloride buffer (PMC: 40\% (w/v) PEG 4000, $10 \mathrm{mM}$ MOPS, $0.6 \mathrm{M}$ sorbitol, $50 \mathrm{mM} \mathrm{CaCl}$ ). The samples were incubated on ice for half an hour. After addition of $2.5 \mathrm{ml}$ of PMC buffer, the samples were incubated for $20 \mathrm{~min}$ at room temperature. Before centrifugation $(2162 \times \mathrm{g}, 15 \mathrm{~min}, 4$ $\left.{ }^{\circ} \mathrm{C}\right) 20 \mathrm{ml} \mathrm{SMC} \mathrm{buffer}$ was added to the protoplasts. To promote the regeneration of protoplasts, YPG medium containing $0.8 \mathrm{M}$ sorbitol was added to the sedimented cells and incubated for $30 \mathrm{~min}$ at $25{ }^{\circ} \mathrm{C}$. After centrifugation $\left(2162 \times \mathrm{g}, 15 \mathrm{~min}, 4{ }^{\circ} \mathrm{C}\right)$, cells were washed with SMC buffer, then resuspended into $1-2 \mathrm{ml}$ of SMC buffer and 1\% (w/v) agar containing YNB selective medium and pored onto YNB selective medium. The plates were incubated at $25{ }^{\circ} \mathrm{C}$ until $4-7$ days. Transformants were selected on the basis of auxotrophy complementation on YNB solid media supplemented with leucine or uracil, as required.

\subsubsection{Microscopy and examination of mitotic stability of the mutants}

AxioCam ERc 5s (Carl Zeiss) AxioLab (Carl Zeiss) equipped fluorescence microscope was used for microscopic observations and recordings and their evaluation was performed by using ZEN 2011 software (Carl Zeiss). To determine the number of 
germinating spores, hyphae branching and cytoplasmic effusions, we used YNB broth and YNB solid media. Cellophane discs were placed on the surface of solid media then inoculated by dropping of $10^{4}-10^{6}$ sporangiospores, after that incubated at $25{ }^{\circ} \mathrm{C}$ for $4-$ 24 hours. Annexin V-FITC Apoptosis Detection Kit (Sigma) was used following the instruction of the manufacturer to differentiate apoptotic and necrotic germlings (in four and eight hours old cultures in YNB broth). For the detection of propidium iodide staining, filter15 (excitation PB 546/12 nm, emission LP 590 nm, Carl Zeiss) was used and filter set 9 for the annexin V-FITC staining (excitation BP 450-490 nm, emission LP 515, Carl Zeiss).

To determine the CFU, $2 \times 10^{2}$ sporangiospores were spread onto selective YNB medium $(\mathrm{pH} 3.5)$. For the mitotic stability investigation the harvested spores were inoculated onto MEA medium and cultivated for 4 days at $25{ }^{\circ} \mathrm{C}$. The sporangiospores were collected and fresh MEA medium was inoculated. This step was repeated up to twelve time (cycles), and then collected spores were spread onto selective YNB medium.

\subsubsection{Determination of the carotenoid and ergosterol content in M. circinelloides}

Fungal carotenoids were extracted as described earlier (Papp et al., 2006;

Csernetics et al., 2011). The mycelium was disrupted in liquid nitrogen with mortar and pestle and $500 \mu \mathrm{l}$ acetone was added to $500 \mathrm{mg}$ mycelium powder. After vigorous vortexing followed by centrifugation $(3000 \times \mathrm{g}, 1 \mathrm{~min}$, room temperature), the upper phase was collected in fresh tubes. The extraction was performed until the mycelium powder has becomes colorless. The extract was combined with an equal volume of petroleum ether (40 $-70{ }^{\circ} \mathrm{C}$ ). To facilitate the separation $2 \mathrm{ml}$ distilled water was added and then centrifuged $\left(2162 \times \mathrm{g}, 8 \mathrm{~min}, 4{ }^{\circ} \mathrm{C}\right)$. The petroleum ether fraction was collected in a fresh tube and dried under nitrogen. Dried samples were dissolved in $10 \mathrm{ml}$ petroleum ether $\left(40-70{ }^{\circ} \mathrm{C}\right)$ and their total carotenoid content was determined by using spectrophotometer at $450 \mathrm{~nm}$ according to Rodriguez-Amaya and Kimura (Rodriguez-Amaya and Kimura, 2004). The samples were dried again and redissolved in tetrahydrofuran supplemented with butylated hydroxytoluene $(100 \mu \mathrm{g} / \mathrm{ml})$ directly before the HPLC analyzis. Determination of the carotenoid composition of the samples was performed with a modular Shimadzu lowpressure gradient HPLC system, as described earlier (Csernetics et al., 2011).

Ergosterol extraction was performed using the method described by Alcazar Fuoli and coworker with some modifications (Alcazar Fuoli et al., 2008). The fresh mycelium 
dried overnight at $80{ }^{\circ} \mathrm{C}$ was disrupted and the weight was determined. Three $\mathrm{ml} 25 \%$ alcoholic (methanol:ethanol 3:2, v/v) $\mathrm{KOH}$ solution was added to the mycelium powder and the samples were vortexed vigorously for $3 \mathrm{~min}$. Samples were incubated at $85^{\circ} \mathrm{C}$ for 1 hour, followed by extraction with a mixture of $1 \mathrm{ml}$ distilled water and $3 \mathrm{ml}$ hexane. After vigorous vortexing for $3 \mathrm{~min}$, extracts were centrifuged $\left(1900 \times \mathrm{g}, 10 \mathrm{~min}\right.$, at $\left.4{ }^{\circ} \mathrm{C}\right)$. The upper layer (liquid phase) was transferred to a clean plastic tube and the liquid phase was evaporated under nitrogen gas. Dried samples were redissolved in $1 \mathrm{ml}$ methanol for HPLC analyzis. For HPLC a modular Shimadzu system equipped with a two channel UV/VIS detector was used, and $50 \mu 1$ sample was subjected on a Prodigy C18 $(4.6 \times 250 \mathrm{~mm}$, ODS 5 $\mu \mathrm{m})$ column (Phenomenex). Isocratic separation was performed with $\mathrm{H}_{2} \mathrm{O}$ /methanol (2:98, $\mathrm{v} / \mathrm{v}$ ) as mobile phase at a flow rate of $1.2 \mathrm{ml} / \mathrm{min}$. The detection wavelengths were 210 and $280 \mathrm{~nm}$; ergosterol standard was purchased from Sigma (Nagy et al., 2014).

\subsubsection{Antifungal susceptibility test}

Antifungal susceptibility tests were performed following the instructions of the Clinical and Laboratory Standards Institute (CLSI) M38-A2 (2008) protocol with minor modifications. Sensitivity of the fungal strains to AmB, ITR and CLO (Sigma-Aldrich, powder, dissolved in DMSO), FLU (Lescol, Novartis, capsule, dissolved in methanol) and SIM (Vasilip, Egis, tablet, dissolved in methanol) was examined in a 96-well microtiter plate assay.

Isolates were grown on selective YNB medium for 5 days at $25{ }^{\circ} \mathrm{C}$. Sporangiospores were harvested with distilled water and concentration was determined with Bürker-chamber counting; selective YNB broth was used to set the concentration to $1 \times 10^{5}$ spore $/ \mathrm{ml}$.

Before dilution, SIM was activated from its lacton pro-drug form with ethanol$\mathrm{NaOH}$ hydrolysis $(15 \% \mathrm{v} / \mathrm{v}$ ethanol, $0,25 \%(\mathrm{v} / \mathrm{v}) \mathrm{NaOH}$, the final volume was set with methanol) at $60{ }^{\circ} \mathrm{C}$ for 1 hour (Lorenz and Parks, 1990; Nyilasi et al., 2014). Ten step dilution of the agents was performed in methanol or DMSO following the instructions of the CLSI M38-A2 method, while the final concentration was set with selective YNB media. Ranges of the final concentration of agents in two-fold dilutions were as follows: AmB: $0.03125-16 \mu \mathrm{g} / \mathrm{ml}$, ITR: $0.125-128 \mu \mathrm{g} / \mathrm{ml}$, CLO: $0.0625-32 \mu \mathrm{g} / \mathrm{ml}$, FLU and SIM: $0.125-64 \mu \mathrm{g} / \mathrm{ml}$.

$100-100 \mu 1$ of medium containing antifungal agent (in double concentration) and medium containing $1 \times 10^{5}$ spore/ml spores $\left(1 \times 10^{4}\right.$ spores/well in final concentration) were 
subjected into the wells. Growth in antifungal agent-free medium supplemented with solvent was considered as $100 \%$ of growth, while uninoculated medium was used as the background for the calibration and contamination control. Plates were incubated for 48 hours at $25{ }^{\circ} \mathrm{C}$ and the optical density of the fungal cultures was measured at $620 \mathrm{~nm}$ with a SPECTROstar Nano (BMG Labtech) plate reader in well-scanning mode. Minimum inhibitory concentration was considered as the lowest concentration of the agent, which result in equal or above $90 \%$ growth inhibition $\left(\mathrm{MIC}_{90}\right)$ compared to the untreated control. The experiments were performed in biological triplicates and technical duplicates.

\subsubsection{Phagocytosis assay}

Phagocytosis assay was performed by Dr. Árpád Csernetics in Germany (Hans Knöll Institute, Jena, Germany), and the candidate was involved in the data evaluation. Growth maintenance of murine alveolar MH-S macrophages (ATCC: CRL-2019) was carried out by Hans-Martin Dahse (Hans Knöll Institute, Jena, Germany) as described previously (Kraibooj et al., 2014). For phagocytosis assay, macrophages were plated in 24 well plates (NUNC 142475, Thermo Scientific) at a density of $2 \times 10^{5}$ cells/well and allowed to adhere overnight at $37^{\circ} \mathrm{C}$.

For phagocytosis assay the Mucor spores were collected from $5-7$ days old cultures with $1 \times \mathrm{PBS}$ (phosphate-buffered saline: $137 \mathrm{mM} \mathrm{NaCl}, 2.7 \mathrm{mM} \mathrm{KCl}, 10 \mathrm{mM}$ $\left.\mathrm{Na}_{2} \mathrm{HPO}_{4}, 1.8 \mathrm{mM} \mathrm{KH} \mathrm{PO}_{4}(\mathrm{pH} 7.4)\right)$ washed with $10 \mathrm{ml} 1 \times \mathrm{PBS}$ and filtrated throw cell strainer $(40 \mu \mathrm{m}$ pore size, Thermo Scientific). After washing three times with $1 \times$ PBS spores were collected with centrifugation $\left(2160 \times \mathrm{g}, 8 \mathrm{~min}, 4{ }^{\circ} \mathrm{C}\right)$ and stained with $5 \mathrm{ml} 0.1$ $\mathrm{mg} / \mathrm{ml}$ Fluorescein isothiocyanate (FITC, Sigma-Aldrich) dissolved in carbonate buffer (3 $\mathrm{ml} \mathrm{0.1} \mathrm{M} \mathrm{Na}_{2} \mathrm{CO}_{3}, 7 \mathrm{ml} \mathrm{0.1} \mathrm{M} \mathrm{NaHCO}_{3}(\mathrm{pH} 9.5)$ ) for $30 \mathrm{~min}$ at $37{ }^{\circ} \mathrm{C}$ with gentle shaking $(70 \mathrm{rpm})$ in dark. Spores were collected with centrifugation $\left(2160 \times \mathrm{g}, 8 \mathrm{~min}, 4{ }^{\circ} \mathrm{C}\right)$, washed three times with $1 \times$ PBS, and the spore concentration was determined with Thoma chamber. Spores were washed with RPMI-1640 medium (12-167F, Lonza), supplemented with $10 \%(\mathrm{v} / \mathrm{v})$ heat inactivated fetal bovine serum (ATCC-30-2020), 1\% ultraglutamine I (w/v) (17-605E/U1, Lonza), $550 \mu \mathrm{l} / 1$ (50 mg/ml stock solution) gentamicin sulphate (17518Z, Lonza), $0.05 \mathrm{mM}$ 2-mercaptoethanol (31350-010, Life Technologies) and 1\% (w/v) sodium-pyruvate solution (BE13-115E, Lonza)) and $10^{6}$ spores were resuspended in $1 \mathrm{ml}$ pre-warmed RPMI-1640, supplemented with $0.02 \mathrm{~g} / \mathrm{l}$ uracil. The medium of $\mathrm{MH}-\mathrm{S}$ macrophages was discarded and medium containing the FITC-labelled spores was added to the macrophages at multiplicity of infection 5. Synchronization was performed with 
centrifugation $\left(100 \times \mathrm{g}, 5 \mathrm{~min}, 21^{\circ} \mathrm{C}\right)$ and the co-incubation was carried out in a humidified $\mathrm{CO}_{2}$ incubator at $37^{\circ} \mathrm{C}$ for 3 hours. After the incubation, the phagocytosis was stopped with ice-cold $1 \times$ PBS for $2-5 \mathrm{~min}$. The non-phagocytosed spores were counterstained with $0.1 \mathrm{mg} / \mathrm{ml}$ calcofluor white (CFW, Sigma-Aldrich) in $1 \times$ PBS for $15 \mathrm{~min}$ at room temperature. Wells were washed three times with $1 \times$ PBS followed by fixation with $3.7 \%(\mathrm{v} / \mathrm{v}$, in $1 \times \mathrm{PBS})$ formaldehyde at room temperature for $15 \mathrm{~min}$. Microscopic images were taken with IN Cell Analyzer 2200 (GE Healthcare Life Sciences). Ten pictures on different areas of the well were taken from each wells; and three mutants from each were analyzed in three biological and two technical replicates. The calculation of phagocytic indexes (PI) was performed for each pictures, using the following formula:

$\mathrm{PI}=($ percentage of macrophages containing at least one spore $) \times($ total number of spores inside macrophages / number of macrophages containing at least one spore)

\subsubsection{Statistical analyzes}

Data are presented as mean \pm SD for experiment performed in three biological and two technical replicates. Differences between the groups were assessed by one-way ANOVA and $t$ test unpaired using GraphPad Prism version 5.01 and Microsoft excel software package for Windows and MacBook. A probability $(P)$ value less than $(*) \leq 0.05$, $(* *) \leq 0.01,(* * *) \leq 0.001$ and $(* * * *) \leq 0.0001$ were considered as statistically significant. 


\section{RESULTS AND DISCUSSION}

\subsection{Transcription of six genes under different cultivation conditions involved in the mevalonate-isoprenoid biosynthesis in M. circinelloides}

In our study one of the aims was to characterize the $h m g S, m v k, d m d, i p i, i s o A$ and carG genes play role in the $M$. circinelloides mevalonate-isoprenoid pathway (Fig. 4). First of all, the transcription of the genes under different cultivation conditions was analyzed. The experiments were carried out in two biological and two technical parallels. The Mucor actA, encoding actin in $M$. circinelloides was used as reference gene. The reaction conditions were optimized with purified $M$. circinelloides MS12 genomic DNA and $60{ }^{\circ} \mathrm{C}$ was found as an optimal annealing temperature at which all PCR products reached the threshold level with equal Ct values (Fig. 9). Beside, the results of melting curve analyzis (data not shown) also verified that no any aspecific PCR product has been amplified, moreover, together with the previous results of the research group can be concluded that the M. circinelloides MS12 genome contains the examined genes in one copy (unpublished data).

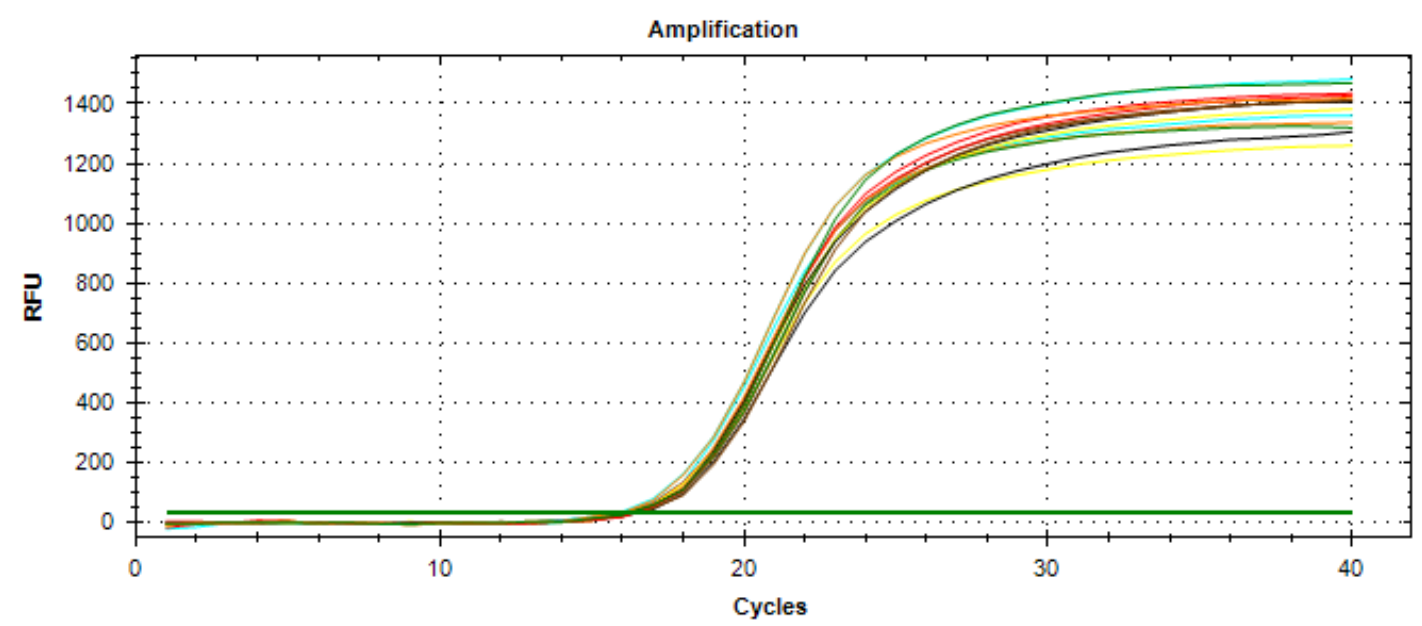

Fig 9. Optimization of the quantitative real-time PCR reaction conditions. The amplification was performed from $M$. circinelloides MS12 DNA with primers designed for qPCR and shown in Table 2. The reaction curves intersect the threshold line at the same $\mathrm{Ct}$ value: $h m g S$ - yellow, $m v k$ - black, $d m d$ - blue, ipi - brown, isoA - red, carG - orange and actA - green.

\subsubsection{Comparison of relative transcription level of selected genes}

The transcription of the six selected genes was compared to each other. In the experiment $M$. ciricinelloides was cultivated in liquid and on solid YNB media supplemented with uracil and leucine up to four days. In all cases, the isoprenoid pathway genes (ipi, isoA and carG) showed higher transcription level than mevalonate pathway 
genes ( $h m g S, m v k$ and $d m d)$. Moreover, we found that $m v k$ showed the lowest, while ipi displayed highest transcription level in comparison to each other at both conditions (Fig. 10).
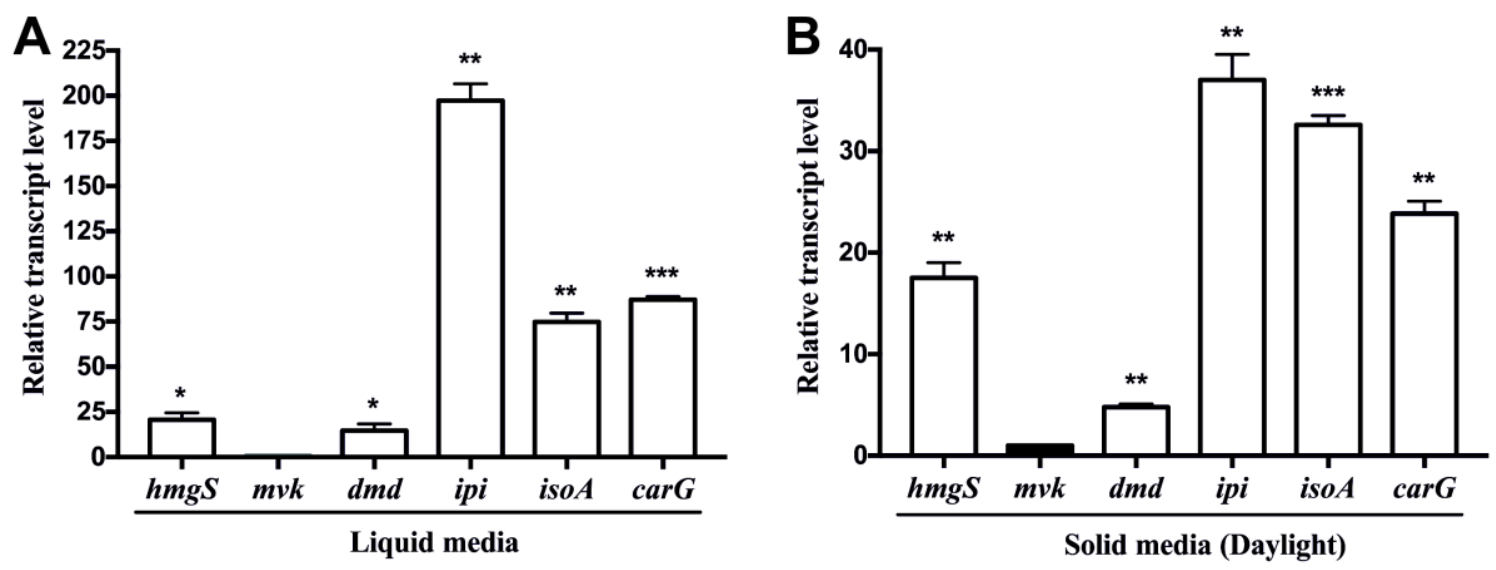

Fig 10. Comparison of the relative transcription levels of the six $M$. circinelloides mevalonateisoprenoid pathway genes to each other: $h m g S$ (HMG-CoA synthase), $m v k$ (mevalonate kinase), $d m d$ (diphosphomevalonate decarboxylase), ipi (IPP isomerase), isoA (FPP synthase) and carG (GGPP synthase) data compared with $m v k$ (mevalonate kinase).

\subsubsection{Effect of environmental conditions on the transcription of selected $M$. circinelloides mevalonate-isoprenoid pathway genes}

Effect of different environmental conditions (such as temperature, light source, incubation time and oxygen tension) on the transcription of the $h m g S, m v k, d m d$, ipi, isoA and $\operatorname{car} G$ genes was analyzed. Temperature dependence of the gene transcription was tested by cultivating the $M$. circinelloides MS12 on solid YNB media supplemented with leucine and uracil at $15{ }^{\circ} \mathrm{C}, 20{ }^{\circ} \mathrm{C}, 25{ }^{\circ} \mathrm{C}, 30{ }^{\circ} \mathrm{C}$ and $35^{\circ} \mathrm{C}$. In general, the fungus is cultivated on the optimal $25^{\circ} \mathrm{C}$ in our experiments. It has been published that an optimum growth temperature range of $25-29^{\circ} \mathrm{C}$, while minimum growth temperature range of -3 to $-4^{\circ} \mathrm{C}$ for Mucor spp. (Sautour et al., 2002; Gougouli et al., 2011). The $35{ }^{\circ} \mathrm{C}$ growth temperature significantly increased the transcription of hmgs, $m v k$, dmd and ipi genes in comparison with the control condition $\left(25^{\circ} \mathrm{C}\right)$. Temperature, lower than the optimum (15 ${ }^{\circ} \mathrm{C}$ and $20{ }^{\circ} \mathrm{C}$ ) also affected the transcription of the genes, the both increased the

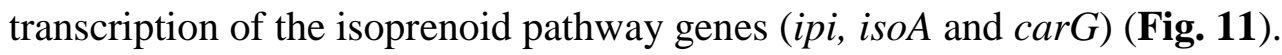



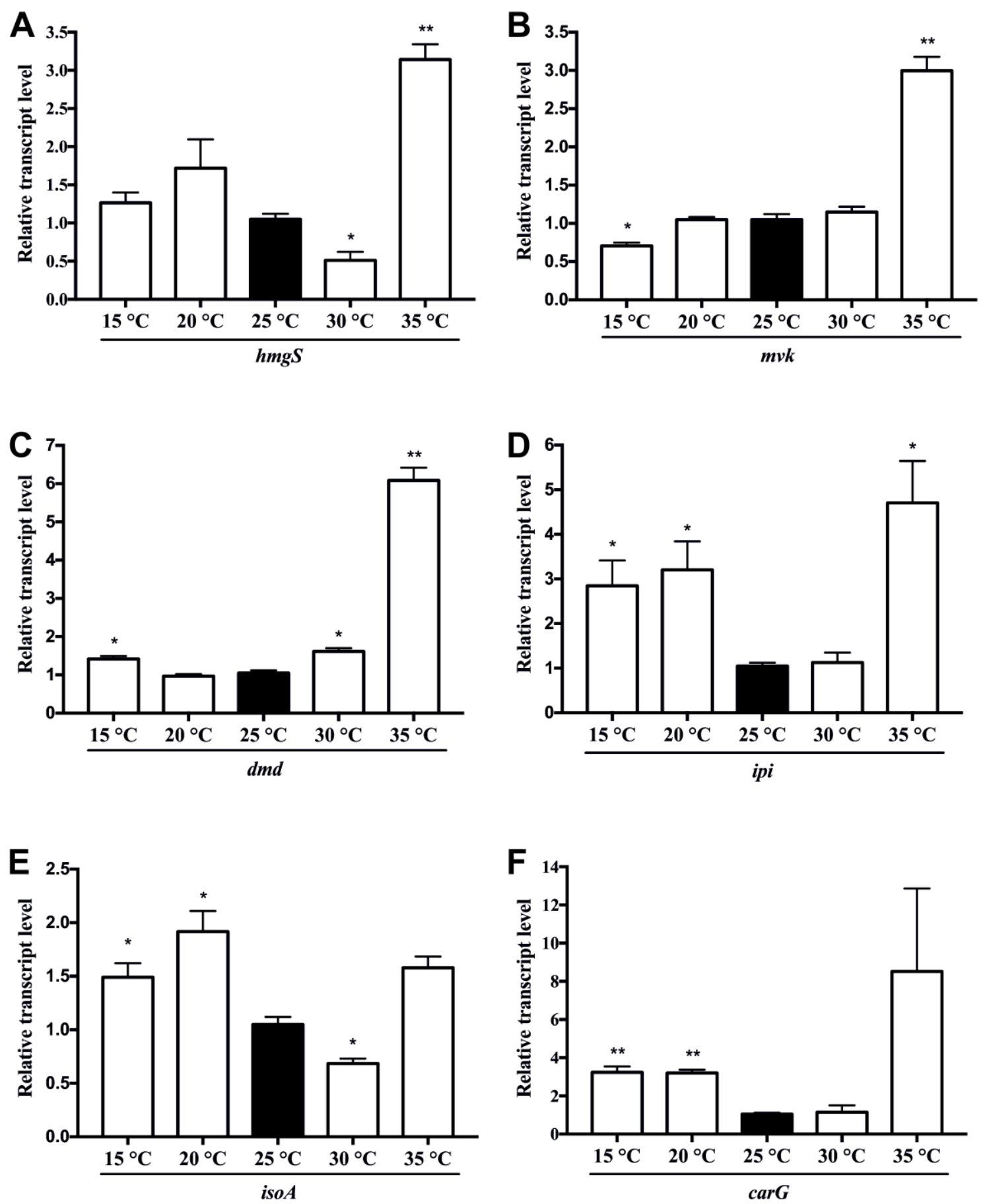

Fig 11. Effect of temperature $\left(15^{\circ} \mathrm{C}, 20^{\circ} \mathrm{C}, 25^{\circ} \mathrm{C}, 30^{\circ} \mathrm{C}\right.$ and $\left.35^{\circ} \mathrm{C}\right)$ on the transcription of the $M$. circinelloides mevalonate-isoprenoid genes: $h m g S$ (HMG-CoA synthase), $m v k$ (mevalonate kinase), $d m d$ (diphosphomevalonate decarboxylase), ipi (IPP isomerase), isoA (FPP synthase) and carG (GGPP synthase).

The cultivation temperature has a strong effect on production of primary and secondary metabolites, such as ergosterol and carotenoid (Mosqueda-Cano and Gutierrez-Corona, 1995; Nagy et al., 2012; Papp et al., 2013). When the cultivation temperature was increased from $28{ }^{\circ} \mathrm{C}$ to $40{ }^{\circ} \mathrm{C}$ the carotenoid content of $M$. rouxii was 
increased three times (Mosqueda-Cano and Gutierrez-Corona, 1995). Also increased carotenoid production and $\beta$-carotene accumulation was observed on $20{ }^{\circ} \mathrm{C}$ and $35{ }^{\circ} \mathrm{C}$ in comparison with cultivation of $M$. circinelloides on $25{ }^{\circ} \mathrm{C}$ (Papp et al., 2013). In our experiments $h m g S, m v k$, dmd and ipi genes showed higher transcription level when the fungus was cultivated on $35^{\circ} \mathrm{C}$ in comparison with $25^{\circ} \mathrm{C}$. This may happen that at higher temperature can accumulate more precursors for $\beta$-carotene accumulation, which may protect the cells against the increased level of endogenous reactive oxygen species (Papp et al., 2013).

Studies have shown that temperature can also influence the ergosterol content of the fungal plasma membrane in Penicillium roqueforti (Li et al., 2009). In psychrophilic yeast Xanthophyllomyces dendrorhous 50\% increment in overall carotenoid content was observed at low temperature with increased levels of astaxanthin (Ducrey Sanpietro and Kula, 1998). Similarly, in case of Dunaliella decreasing the cultivation temperature from $34{ }^{\circ} \mathrm{C}$ to $17{ }^{\circ} \mathrm{C}$ resulted 7.5 -fold increase in the carotenoid content (Orset and Young, 1999). The $M$. circinelloides ipi, isoA and carG genes showed higher transcription at $15{ }^{\circ} \mathrm{C}$ and $20^{\circ} \mathrm{C}$ than on $25^{\circ} \mathrm{C}$ in our study, which can be also due to increased ergosterol and carotenoid accumulation. Similar result was found in case of $h m g R l$ gene, encoding a HMG-CoA reductase in $M$. circinelloides: the transcription of $h m g R l$ increased, when the fungus was cultivated at $15^{\circ} \mathrm{C}$ and $20^{\circ} \mathrm{C}$ than on $25^{\circ} \mathrm{C}$ (Nagy et al., 2014).

Light is one of the essential environmental factors that can regulate several biological processes, including growth, tropism and morphogenesis in fungi (Schumacher et al., 2017). Effect of illumination on the transcription of the M. circinelloides MS12 mevalonate-isoprenoid pathway genes was analyzed. The fungus was cultivated up to four days under daylight light source, in dark, and in continuous dark with exposure to $10 \mathrm{sec}$ light followed by $10 \mathrm{~min}$ dark. The genes ipi and carG showed significantly lower transcription when the fungus was cultivated in continuous dark in comparison with cultures exposed continuously. The short light exposure (light induction) significantly increased the transcription of $m v k, d m d$, isoA and $\operatorname{car} G$ genes in comparison with cultivation in continuous light. When the dark and light induction was compared with each other we found a significant increase in the transcription level of ipi and carG (Fig. 12).

To investigate whether the different light sources may affect the gene transcription, the impact of different white light sources (warm white and daylight) on the transcription of the six genes was examined (daylight was used in all other experiments). Only the transcription of the $i s o A$ and $\operatorname{car} G$ showed significant difference in the case of the two light 
sources. The $\operatorname{car} G$ showed higher transcription level, when the strain was cultivated under daylight, than under warm white source; while the isoA showed higher transcription level, when the M. circinelloides MS12 was cultivated under warm white light source (Fig. 13).
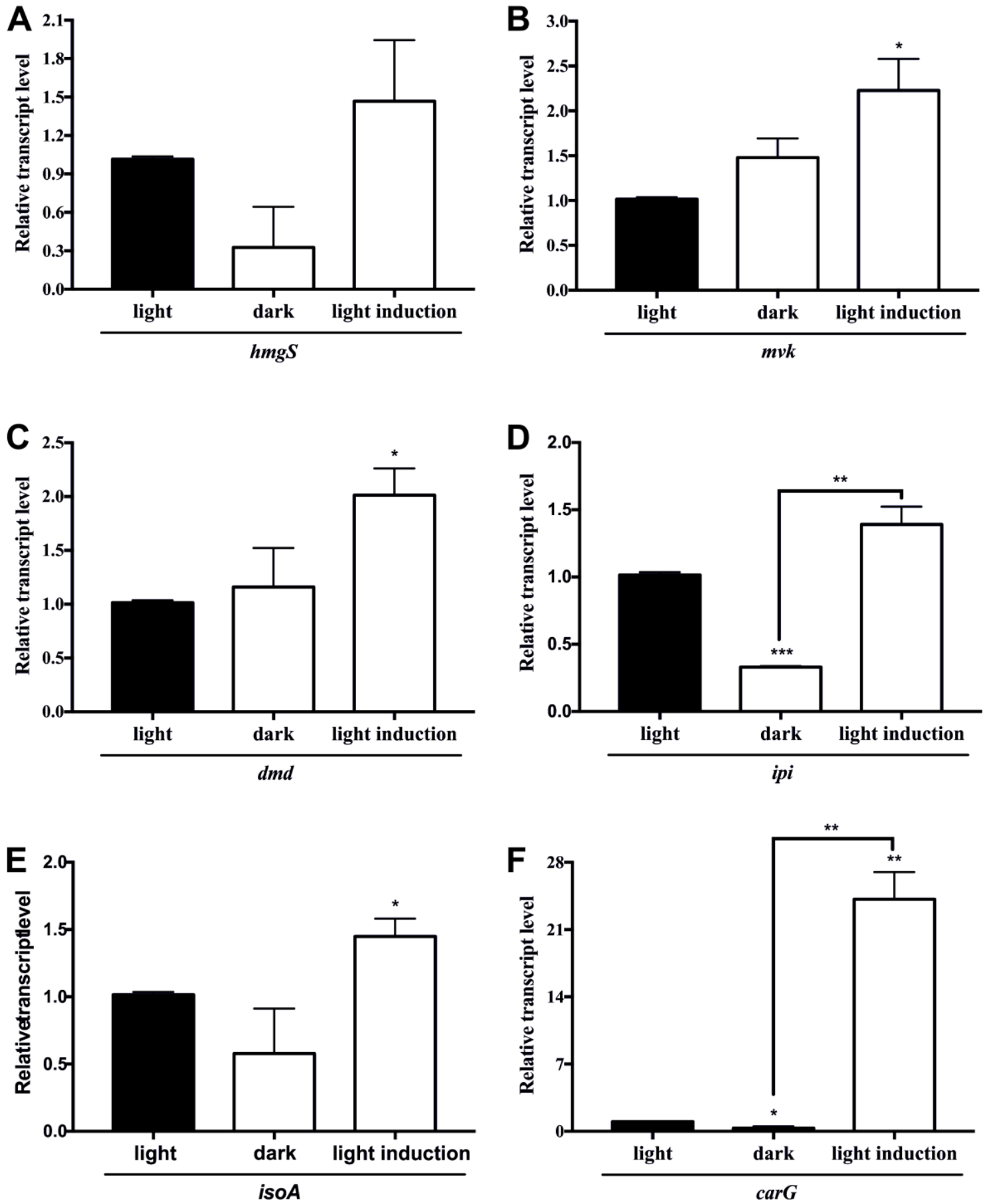

Fig 12. Effect of light (light, dark and dark with following $10 \mathrm{sec}$ light and $10 \mathrm{~min}$ dark (light induction)) on the transcription of M. circinelloides mevalonate-isoprenoid genes: $h m g S$ (HMGCoA synthase), $m v k$ (mevalonate kinase), $d m d$ (diphosphomevalonate decarboxylase), ipi (IPP isomerase), isoA (FPP synthase) and carG (GGPP synthase). 

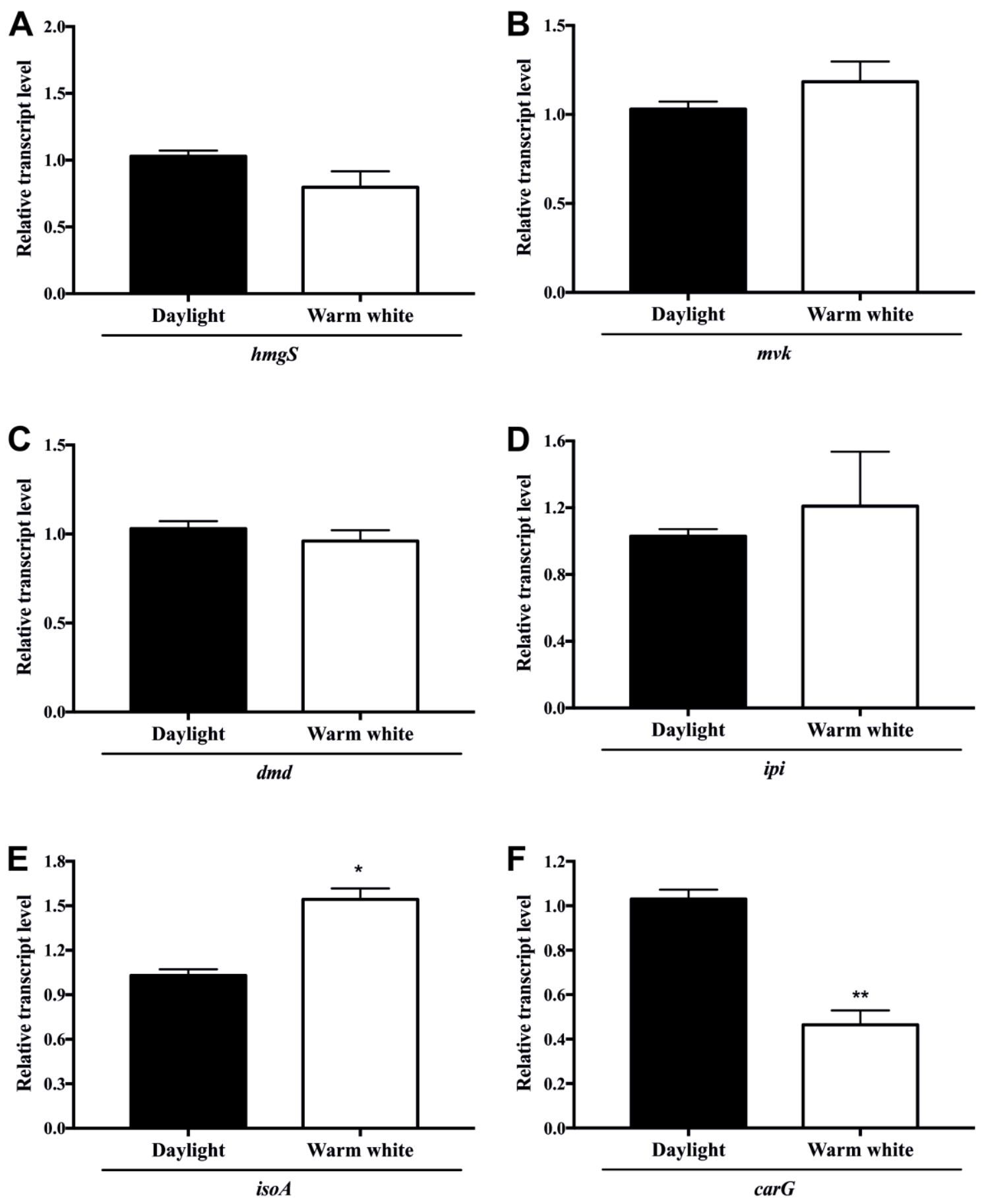

Fig 13. Effect of different light sources (daylight and warm white) on the transcription of $M$. circinelloides mevalonate-isoprenoid genes: $h m g S$ (HMG-CoA synthase), $m v k$ (mevalonate kinase), $d m d$ (diphosphomevalonate decarboxylase), ipi (IPP isomerase), isoA (FPP synthase) and carG (GGPP synthase).

The genes involved in the carotenoid biosynthesis had been shown to be positively photo-regulated not only in fungi, but also in bacteria and tomato plant (Sandmann 1994; Velayos et al., 2000a). $M$. rouxii accumulated about ten times more $\beta$-carotene when grown continuously in the presence of light than corresponding cultures grown in the dark 
(Mosqueda-Cano and Gutierrez-Corona 1995). The transcription of the carB gene (encoding the phytoene dehydrogenase) in M. circinelloides reached up to 150-fold higher level in light than in dark (Velayos et al., 2000a). The promoter region, common for both the $\operatorname{carB}$ and $\operatorname{carRP}$ (encoding the phytoene synthase/lycopene cyclase) genes contains several APE (al-3 proximal element)-like sequences that are involved in light regulation of gene expression (Velayos et al., 2000b). Genes encoding GGPP synthase have been isolated from several fungi: $N$. crassa, Nigrospora sphaerica and M. circinelloides, while in G. fujikuroi two genes which are thought to be involved in different branches of the isoprenoid pathways, have been isolated (Tudzynski and Hölter, 1998; Iturriaga et al., 2000). At least in $N$. crassa and $M$. circinelloides, it has been shown that light induces the expression of gene encoding GGPP synthase (Morelli et al., 1993; Velayos et al., 2003, 2004). However, this photocarotenogenic response is not universal in other fungi such as $B$. trispora, light has no effect on the carotenoid production (Sutter, 1970; Linden et al., 1997). In our study significant increase was observed in the transcription of $m v k, d m d$, isoA and $\operatorname{car} G$ genes with short light induction in comparison with cultivation in continuous light.

The transcription of $c a r G$ after blue light irradiation showed that the expression of this gene is up-regulated by blue light, as happens with the other structural genes ( $\operatorname{car} B$ and carRP) involved in carotenogenesis in $M$. circinelloides, which presume the similar regulation of these genes (Velayos et al., 2003). Navarro and co-workers reported that $M$. circinelloides responds to blue light by activating carotenoid biosynthesis due to a rapid increase in the level of transcription of structural genes for carotenogenesis (Navarro et al., 2001). Mucoromycotina fungi were incubated in dark and under different types of light sources, cultures of certain fungi such as those of Amylomyces rouxii, M. circinelloides and Muсо тисеdo, incubated in light with higher color temperature (daylight) were able to survive at $37^{\circ} \mathrm{C}$, which proved to be lethal if they were growing in dark or in light with lower color temperature (warm white) (Nagy et al., 2012). In our study cultivation of the M. circinelloides under daylight light source, which contains more components in the blue range, resulted significantly higher transcription of $\operatorname{car} G$ in comparison with warm white light source. At the same time interestingly, the isoA showed higher transcription level, when the M. circinelloides was cultivated under warm white light source (Fig. 13).

After spore inoculation, the morphology of $M$. circinelloides sporangiospores significantly changes: four hours after the inoculation the germ tubes are developed, while hyphal branches appear at about 8 hours old cultures. The transcription of the six 
mevalonate-isoprenoid biosynthesis genes was analyzed after different cultivation time (4, 8, 24, 48 and 96 hours old cultures). The mevalonate pathway genes ( $h m g S, m v k$ and $d m d$ ) showed the highest transcription level at four hours after inoculation, which decreased by the time. The highest transcription levels of $i s o A$ at eight hours, while of $\operatorname{car} G$ at 96 hours postinoculation were observed (Fig. 14). These results suggest that precursors of all isoprenoids are synthesized and accumulated in germlings, while those that are responsible for carotenoid production are present mainly in 96 hours old mycelia.

Effect of cultivation time on the transcription of $M$. circinelloides $h m g R l, h m g R 2$ and $h m g R 3$ genes was analyzed. During the cultivation period, the transcription level of $h m g R 2$ and $h m g R 3$ showed similar patterns and both reached high level at four hours postinoculation. More over transcription of $h m g R 2$ showed the highest transcription level in eight hours cultures, while $h m g R l$ and $h m g R 3$ reached their maximum at 48 hours after the inoculation (Nagy et al., 2014).

Morphological dimorphism is a characteristic feature of $M$. circinelloides. In the absence of oxygen and/or presence of high hexose concentration in the medium, filamentous growth of the fungus switches to a yeast-like form. Effect of oxygen tension on the transcription of the mevalonate-isoprenoid genes was analyzed under aerobic, anaerobic and anaerobic followed by one hour aerobic incubation condition. The transcription of six genes increased under anaerobic compare to aerobic conditions. Anaerobic condition followed by one hour aerobic exposure increased the transcription of $d m d$ and significantly decreased the transcription of $\operatorname{car} G$ gene compared to cultures under continuous anaerobic cultivation, while no any significant change could be observed in the case of $h m g S, m v k, i p i$ and isoA genes (Fig. 15).

Productions of isoprenoids are highly regulated by oxygen tension. Ergosterol and carotenoid biosynthesis are oxygen dependent processes (Mantzouridou et al., 2002; C`ertík et al., 2005; Iigusa et al., 2005; Galea and Brown, 2009). Studies have shown that carotenoid biosynthesis is stimulated by aeration in Rhodotorula spp. (Simova et al., 2004). The synthesis of astaxanthin was significantly increased when Phaffia strains were cultivated in media with optimum amount of oxygen (C`ertík et al. 2005). Biosynthesis of ergosterol has high oxygen request and administrative impact of low-oxygen conditions on the transcription of the $h m g R$ gene has also been demonstrated in S. cerevisiae, Aspergillus fumigatus, Cryptococcus neoformans and Schizosaccharomyces pombe (Bien and Espenshade, 2010). In spite of the increased transcription level of mevalonate pathway gene $h m g R 3$, the whole average ergosterol content radically decreased in the anaerobically 
grown cells $(0.2 \mathrm{mg} / \mathrm{g}$ [dry weight]) compared to that of the aerobically grown mycelium (5.5 mg/g [dry weight]) (Nagy et al., 2014). Similar situation was previously observed in Mucor genevensis (Gordon, et al., 1970), which had $<0.3$ and $3 \mathrm{mg} / \mathrm{g}$ [dry weight] ergosterol content during anaerobic and aerobic growth, respectively.
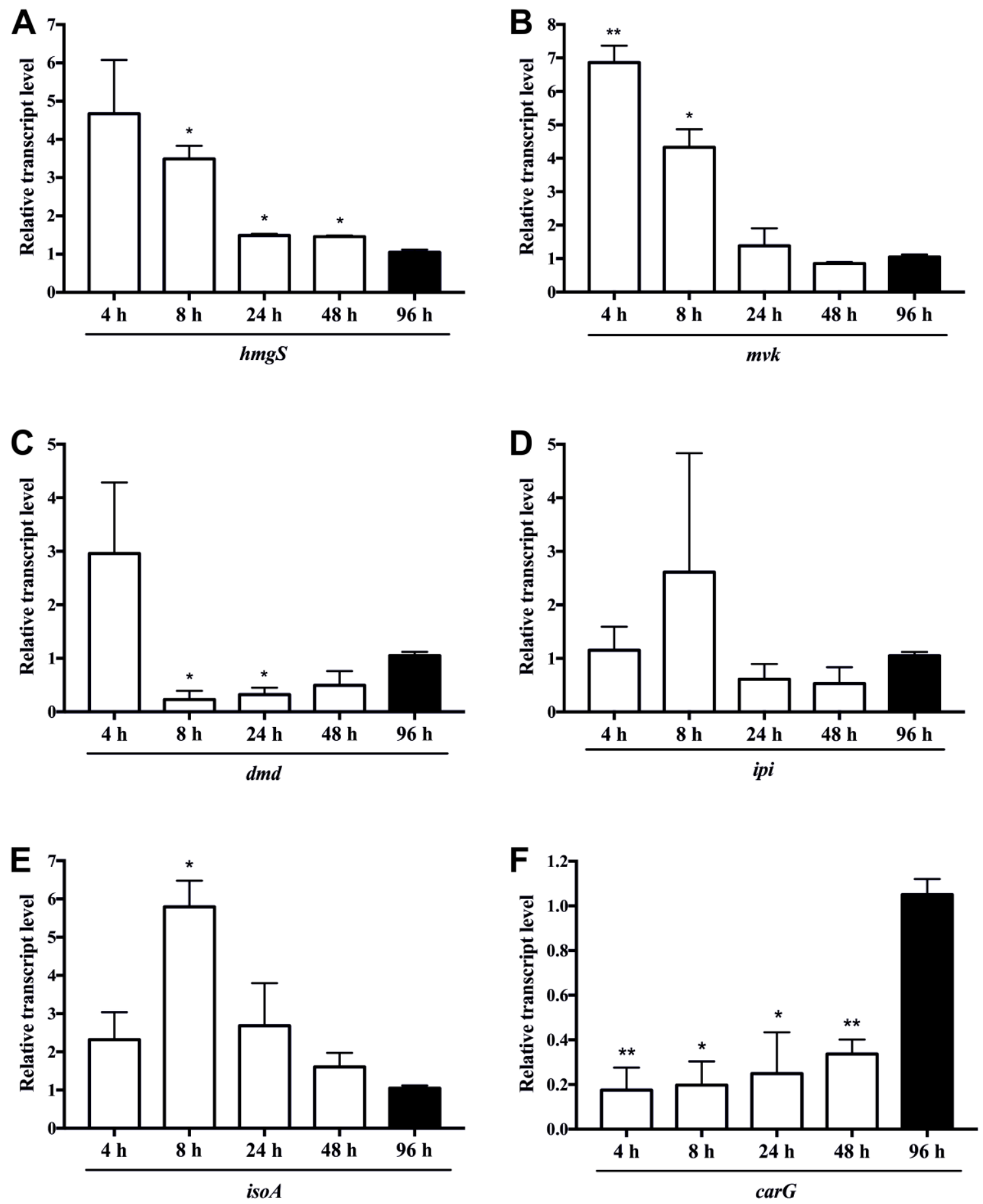

Fig 14. Kinetics of transcription of the $M$. circinelloides mevalonate-isoprenoid pathway genes: $h m g S$ (HMG-CoA synthase), $m v k$ (mevalonate kinase), dmd (diphosphomevalonate decarboxylase), ipi (IPP isomerase), isoA (FPP synthase) and carG (GGPP synthase). Gene transcription was analyzed in 4 h, 8 h, 24 h, $48 \mathrm{~h}$ and $96 \mathrm{~h}$ old cultures and all data are compared to 96 hour cultures. 

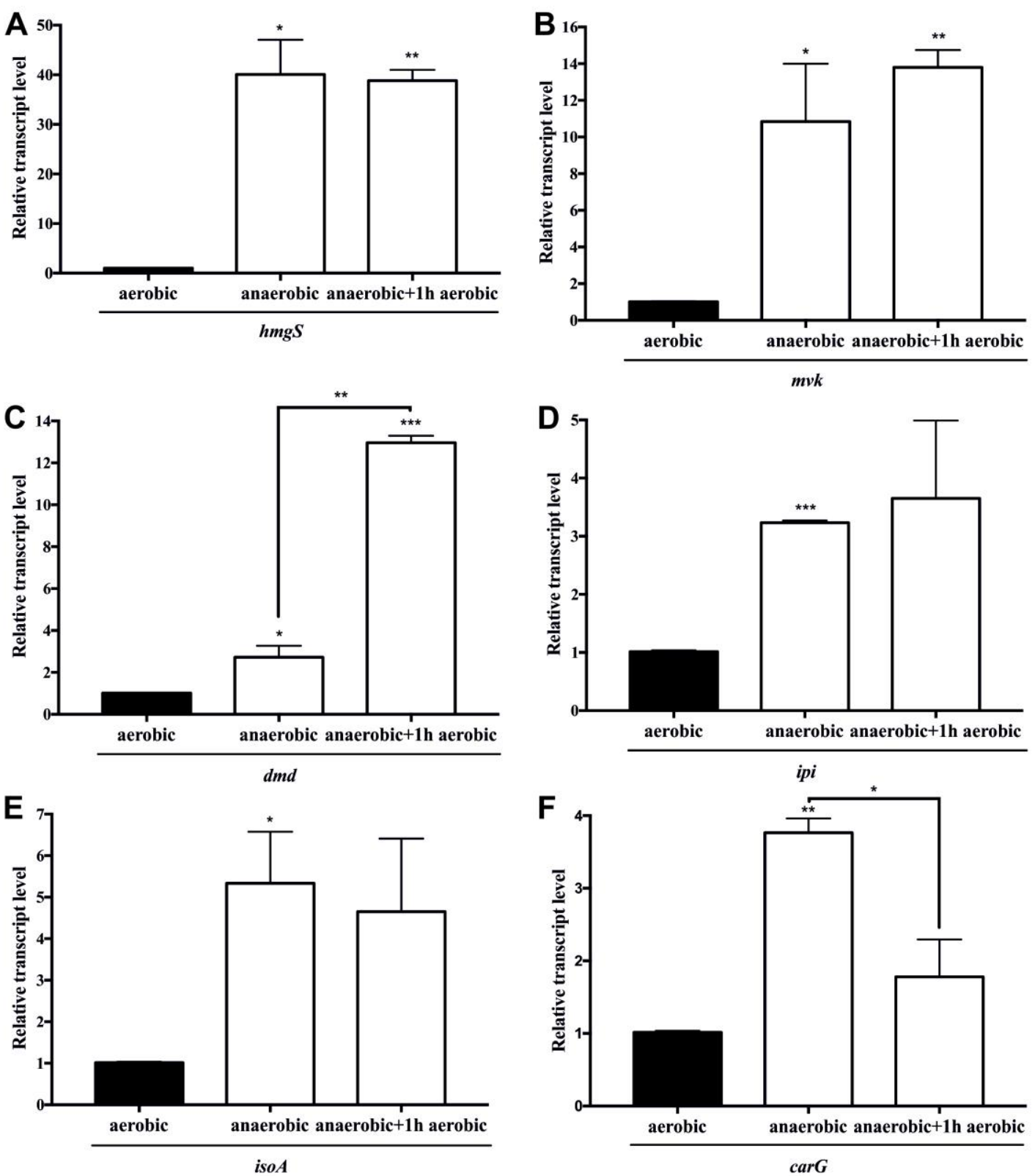

Fig 15. Effect of oxygen tension (aerobic, anaerobic and anaerobic followed by 1 hour aerobic exposure) on the transcription of $M$. circinelloides mevalonate-isoprenoid pathway genes: $h m g S$ (HMG-CoA synthase), mvk (mevalonate kinase), dmd (diphosphomevalonate decarboxylase), ipi (IPP isomerase), isoA (FPP synthase) and $\operatorname{carG}$ (GGPP synthase).

\subsubsection{Effect of medium composition on the transcription of the $M$. circinelloides six} mevalonate-isoprenoid pathway genes

Next, effect of the composition of the cultivation medium (such as different carbon sources, glucose concentration, addition of sodium chloride) on the transcription of the hmgS, $m v k$, dmd, ipi, isoA and carG genes was investigated in $M$. circinelloides. To determine the effect of medium composition on the transcription of the genes, $M$. 
circinelloides was grown on minimal YNB medium supplemented with leucine and uracil and complete MEA medium. We found that relative transcription level of $m v k$ and isoA significantly decreased, whereas $\operatorname{car} G$ significantly increased on MEA in comparison to YNB, while no any significant changes were observed in the case of the other genes (Fig. 16). The exact composition of MEA medium, as it contains yeast- and malt extract, is not known, thus the effect of several compounds (e.g. salts, carbon sources) may result in difference in transcription levels in comparison with the defined YNB.
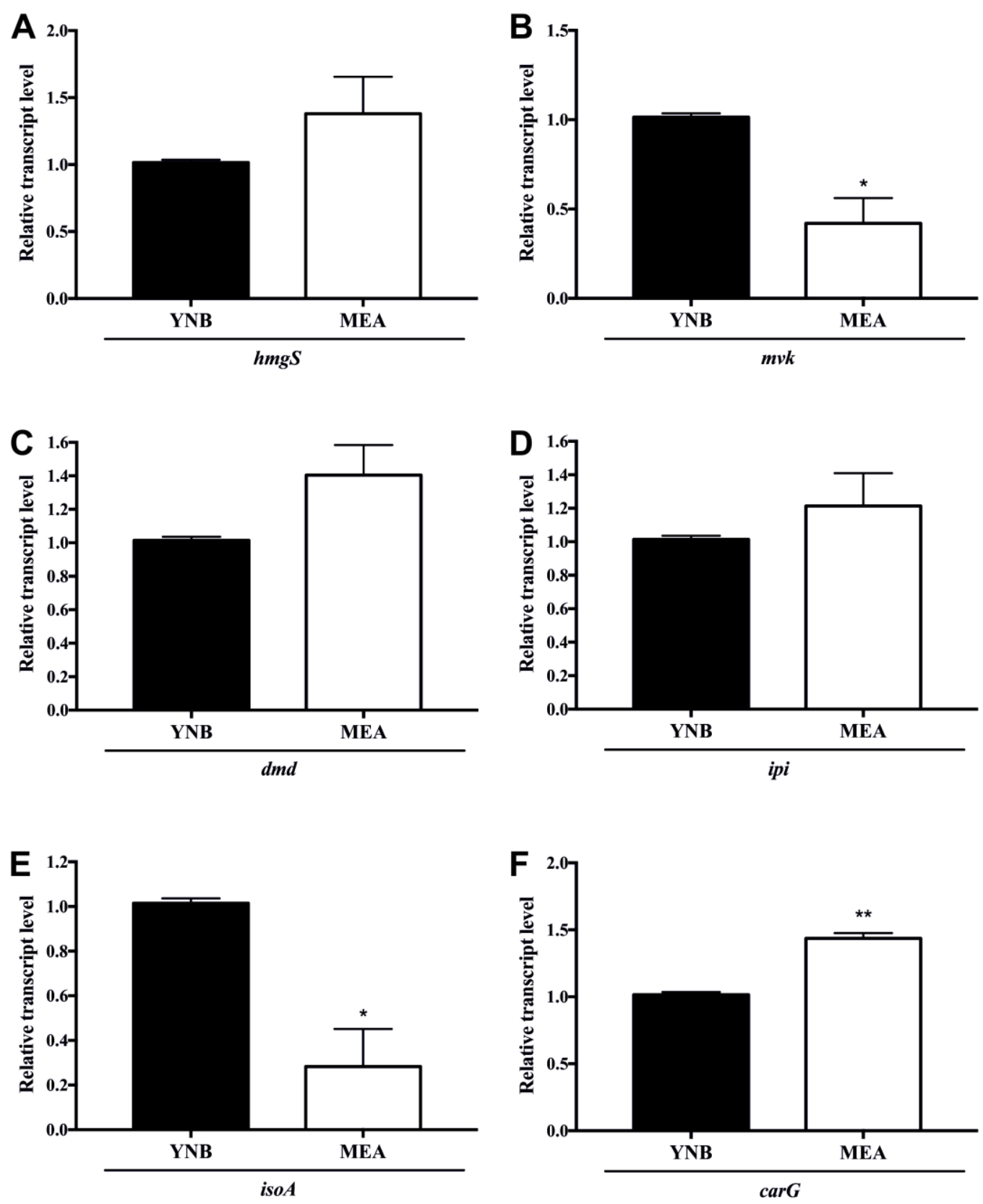

Fig 16. Effect of the minimal (YNB) and complete (MEA) media on the transcription of the $M$. circinelloides mevalonate-isoprenoid biosynthesis pathway genes: hmgS (HMG-CoA synthase), $m v k$ (mevalonate kinase), dmd (diphosphomevalonate decarboxylase), ipi (IPP isomerase), isoA (FPP synthase) and carG (GGPP synthase). 
Glucose is the most widely utilizable carbon source, and it is known, that glucose affect several biochemical processes. To investigate the effect of glucose concentration on the gene transcription, glucose was added to YNB medium in a final concentration of $1 \%$, $2.5 \%$ and $5 \%(\mathrm{w} / \mathrm{v})$ and cultivation was carried out for four days under daylight light source at $25{ }^{\circ} \mathrm{C}$. Significant decreases in the transcript levels of the ipi and isoA on both concentrations, in the case of $h m g S$ on $5 \%$ (w/v) glucose only, and in case of carG at $2.5 \%$ (w/v) glucose was observed (Fig. 17). Effect of the glucose concentration on the six gene transcription was also investigated at eight hours after inoculation; in that case the $M$. circinelloides MS12 was cultivated in YNB broth. In this case cultivation in $2.5 \%$ and 5\% $(\mathrm{w} / \mathrm{v})$ glucose containing media resulted decrease in the transcription of all six genes in comparison with $1 \%(\mathrm{w} / \mathrm{v})$ glucose (Fig. 18). Similar decrease was observed in the transcription of ipi, isoA and carG, when M. circinelloides MS12 was cultivated on 2.5\% and $5 \%(\mathrm{w} / \mathrm{v})$ glucose containing media in comparison with $1 \%(\mathrm{w} / \mathrm{v})$ glucose after 96 hours incubation (Csernetics et al., 2011). The results suggesting that increased glucose concentration affect the production of metabolites synthesized via the mevalonateisoprenoid pathway in $M$. circinelloides.

It had been shown that carotenoid biosynthesis is repressed by glucose in $X$. dendrorhous, moreover low carotenoid accumulation was detected, when high glucose concentration was used in the medium (Marcoleta et al., 2011). Besides, it was recently reported that the catabolic repressor Mig1 contribute to the regulation of carotenoid accumulation in $X$. dendrorhous (Alcaíno et al., 2016). Possible glucose-dependent regulation of ERG10A and ERG1OB genes was studied by Werner and coworkers in $X$. dendrorhous (Werner et al., 2016). The transcription level of ERG10A (encodes a thiolase involved in the mevalonate pathway) did not show any significant changes after the addition of glucose. In contrast, transcription of ERG1OB (thiolase involved in the $\beta$ oxidation of fatty acids) reduced approximately 120-fold when glucose was added to the culture compared to the untreated control (Werner et al., 2016). However, this decrease was only temporary as the transcription levels normalized to control levels when the glucose in the media was consumed. Similarly, in our study, on $2.5 \%$ and $5 \%(\mathrm{w} / \mathrm{v})$ glucose more prominent decrease was detected in the transcription of all six genes after 8 hours cultivation than after 96 hours, moreover no significant decrease was observed in the latter case in the transcription of $m v k$ and $d m d$ genes in comparison with $1 \%(\mathrm{w} / \mathrm{v})$ glucose, which can be due to glucose consumption (Fig. 17 and Fig. 18). 

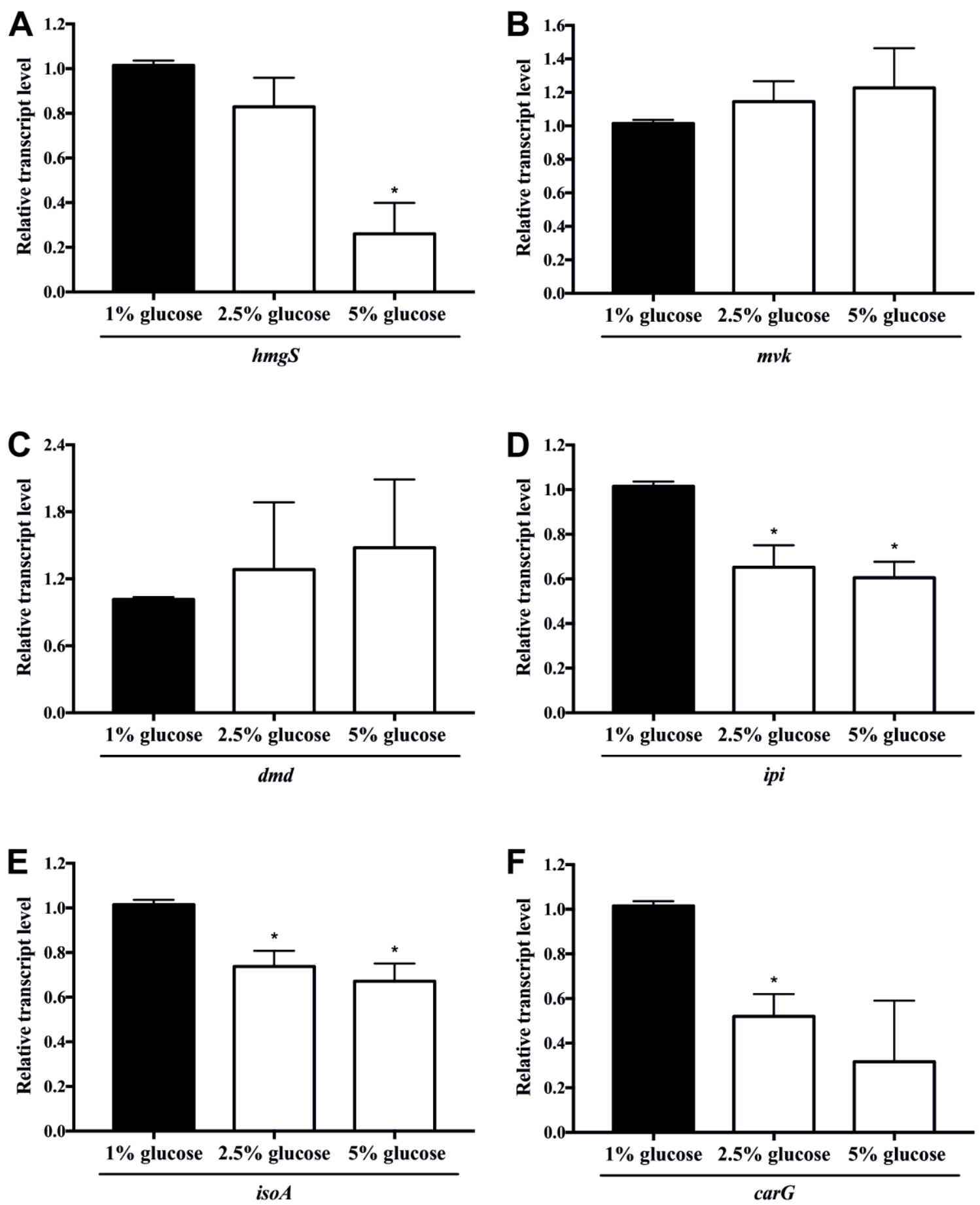

Fig 17. Effect of the glucose concentration on the transcription of the M. circinelloides mevalonateisoprenoid genes after four days incubation: $h m g S$ (HMG-CoA synthase), $m v k$ (mevalonate kinase), $d m d$ (diphosphomevalonate decarboxylase), ipi (IPP isomerase), isoA (FPP synthase) and carG (GGPP synthase). 

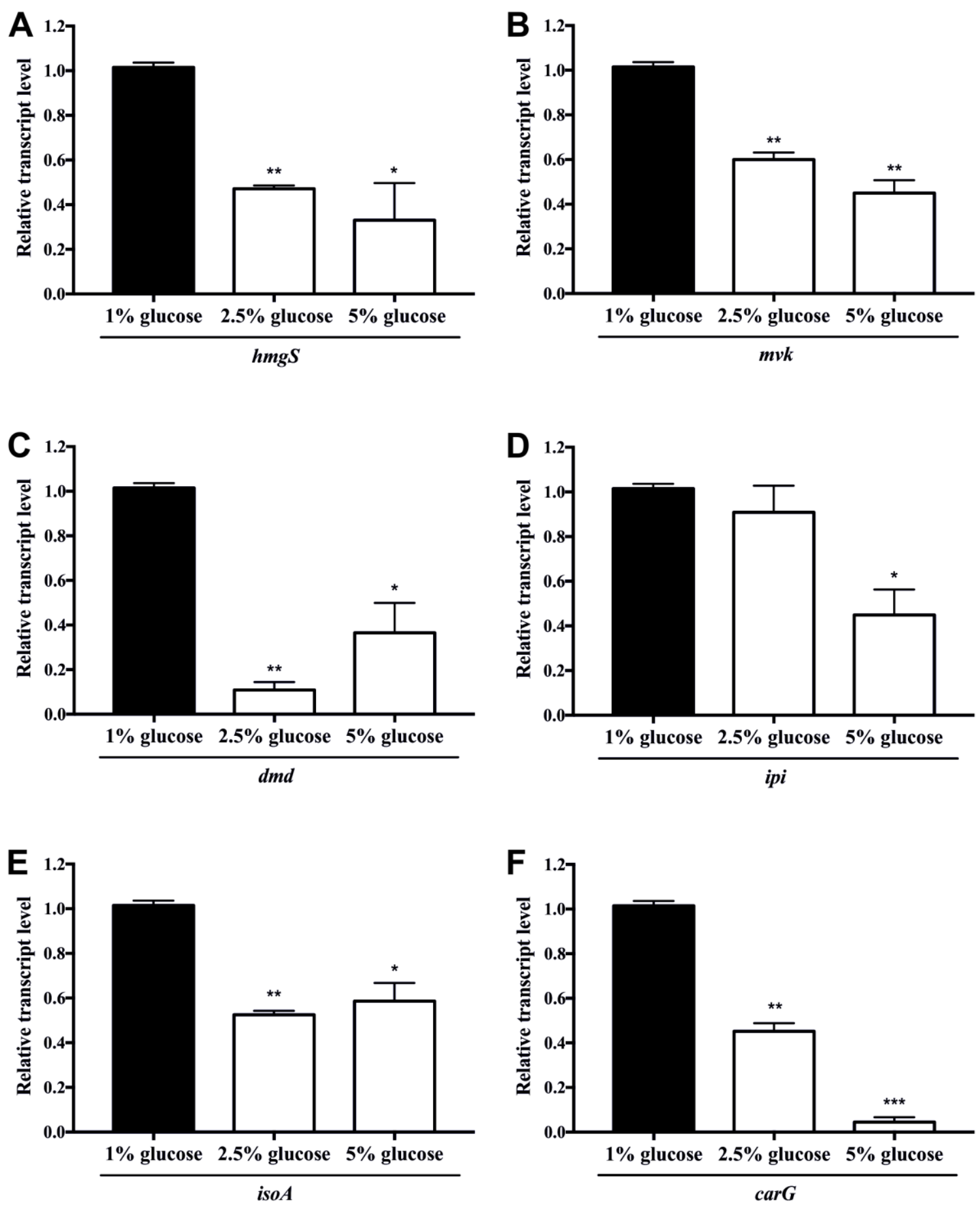

Fig 18. Effect of the glucose concentration on the transcription of the M. circinelloides mevalonate-isoprenoid genes after eight hours cultivation: $h m g S$ (HMG-CoA synthase), $m v k$ (mevalonate kinase), $d m d$ (diphosphomevalonate decarboxylase), ipi (IPP isomerase), isoA (FPP synthase) and $\operatorname{carG}$ (GGPP synthase).

In order to examine the effect of different carbon sources on the transcription of the six mevalonate-isoprenoid pathway genes, glucose was replaced by dihydroxyacetone (DHA), maltose (Mal), sodium acetate (Na-ac) or trehalose (Treh) in YNB medium at a 
final concentration $1 \%(\mathrm{w} / \mathrm{v})$. The relative transcription levels normalized to glucose are shown in Fig. 19.
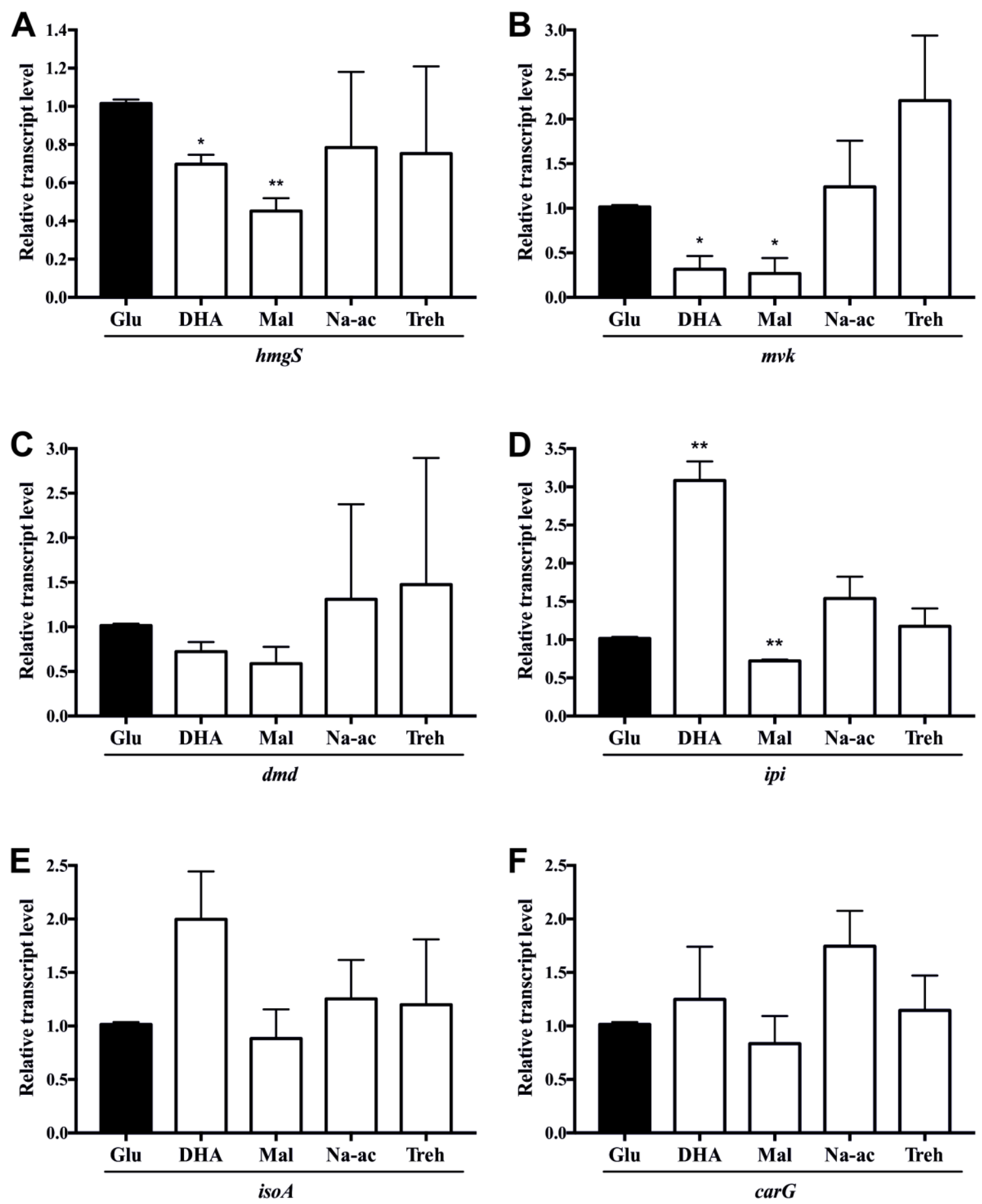

Fig 19. Effect of the different carbon sources on $M$. circinelloides mevalonate-isoprenoid biosynthesis pathway gene transcription: $h m g S$ (HMG-CoA synthase), $m v k$ (mevalonate kinase), $d m d$ (diphosphomevalonate decarboxylase), ipi (IPP isomerase), isoA (FPP synthase) and carG (GGPP synthase). Dihydroxyacetone (DHA), maltose (Mal), sodium acetate (Na-ac), or trehalose (Treh) in a final concentration $1 \%(\mathrm{w} / \mathrm{v})$ was used in YNB media. 
The carbon sources were selected on the basis of previous results of our research group, in which effect of several carbon sources on the carotenoid production of $M$. circinelloides was analyzed (Nagy et al., 2012; Papp et al., 2013). DHA and Mal resulted in significant change in the transcription of $h m g S, m v k$ and ipi genes in comparison with cultivation on glucose containing medium. DHA and Mal significantly reduced the transcription level of $h m g S$ and $m v k$, while in the case of ipi Mal reduced and DHA increased it in comparison with cultivation of the $M$. circinelloides MS12 on glucose containing YNB. Treh and DHA increased the total carotenoid content of several Mucoromycotina fungi, besides, increased the canthaxanthin content in $M$. circinelloides mutants harbouring a bacterial $\beta$-carotene ketolase encoding $c r t W$ gene (Nagy et al., 2012; Papp et al., 2013). Interestingly, Treh had no any significant effect on the transcription of the investigated genes, while DHA reduced the transcription of $h m g S$ and $m v k$, but significantly increased that of ipi. The latter is responsible for formation of DMAPP, a building block of all isoprenoids. DHA take part in the glycolysis and thus, may provide precursors for the mevalonate pathway (Nagy et al., 2014). The $h m g R l$ and $h m g R 3$ genes of $M$. circinelloides (encoding HMG-CoA reductases) displayed increased transcription level on Na-ac and DHA containing medium in comparison with glucose, moreover, relative transcription level of all three $h m g R$ genes on Mal and Treh decreased in comparison with glucose (Nagy et al., 2014). Acetate, as carbon source showed affect on terpenoid biosynthesis in Blakesleea and Phycomyces (Kuzina and Cerdá-Olmedo, 2007).

Effect of the salt stress on the transcription of $M$. circinelloides six mevalonateisoprenoid pathway genes was also analyzed. In this experiment $\mathrm{NaCl}$ was added to the YNB media in $1 \%, 2 \%$ and $3 \%(\mathrm{w} / \mathrm{v})$ final concentration. Interestingly, in our study $\mathrm{NaCl}$ did not affected significantly the gene transcriptions; addition of 2 or $3 \%(\mathrm{w} / \mathrm{v}) \mathrm{NaCl}$ to the media slightly, but not significantly decreased the transcription of the $h m g S$, but $2 \%(\mathrm{w} / \mathrm{v})$ $\mathrm{NaCl}$ significantly increased the transcription of the carG (Fig. 20). The carG gene is responsible for the formation of GGPP a precursor of gernylgeranylated proteins. This protein prenylation is a posttranslational modification and essential for the function by over approximately 100 proteins in the eukaryotic cell (Terry et al., 2006; DeGraw et al., 2012). In the presence of $\mathrm{NaCl}$ modification of the quantity and the composition of sterols in the cell membrane is an important factor of the adaptation to the environmental osmotic changes (Rep et al., 2000; Yancey, 2005). In previous studies, the $h m g R$ found to be essential for the adaptation of certain fungi to the changing salinity of the environment (Bidle et al., 2007; Vaupotič et al., 2008; Nagy et al., 2014). The three $h m g R$ genes of $M$. 
circinelloides showed increase transcription level in the presence of $\mathrm{NaCl}$ (Nagy et al., 2014). HMG-CoA reductase of the extremely halotolerant Hortaea werneckii, HwHmg2, is up-regulated under hypo-saline and extremely hyper-saline conditions, while activity of HwHmg1 is constant under different growth conditions (Vaupotic and Plemenitas, 2007).
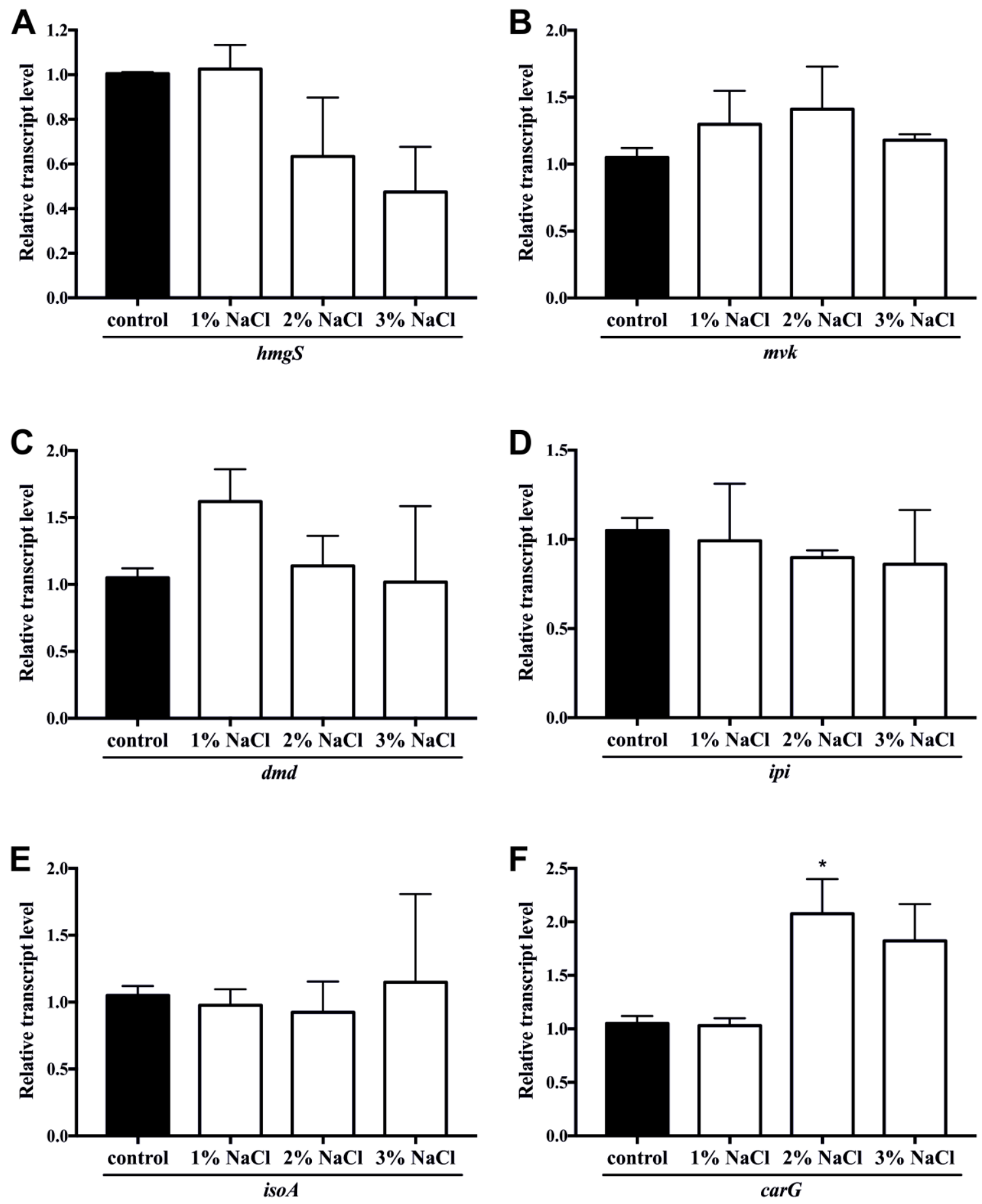

Fig 20. Effect of different concentration of sodium chloride $(\mathrm{NaCl})$ on the transcription of $M$. circinelloides mevalonate-isoprenoid genes: $h m g S$ (HMG-CoA synthase), $m v k$ (mevalonate kinase), $d m d$ (diphosphomevalonate decarboxylase), ipi (IPP isomerase), isoA (FPP synthase) and carG (GGPP synthase). 


\subsubsection{Effect of statin treatment on the transcription of the $M$. circinelloides six mevalonate-isoprenoid pathway genes}

Statins are cholesterol-lowering drugs and competitive inhibitors of HMG-CoA reductases and they induce apoptosis-like process in organisms (Qi et al., 2013). Previously our research group investigated on the susceptibility of $M$. circinelloides against different statins, such as atorvastatin, fluvastatin, rosuvastatin, simvastatin and pravastatin in range $0.125-64 \mu \mathrm{g} / \mathrm{ml}$. Based on the results FLU was selected to investigate on the effect of statins on the transcription of the M. circinelloides mevalonate-isoprenoid six genes. The fungus was cultivated in liquid YNB medium containing FLU in 0.5, 1, 2 and 4 $\mu \mathrm{g} / \mathrm{ml}$ concentration up to four days at $25{ }^{\circ} \mathrm{C}$. Addition of FLU to the medium significantly increased the transcription levels of all six genes in comparison with cultivation in FLUfree medium. At the same time $4 \mu \mathrm{g} / \mathrm{ml}$ FLU concentration (which is the MIC 90 to the fungus determined in that experiment) resulted significant decrease in the transcription levels in comparison to treatment with $2 \mu \mathrm{g} / \mathrm{ml} \mathrm{FLU} \mathrm{(Fig.} \mathrm{21).}$

In eukaryotic organisms, statins inhibit the class I HMG-CoA reductases; as an outcome, it can inhibit the formation of mevalonate from HMG-CoA. Mevalonate is a precursor for other non-steroidal isoprenoid compounds as well, such as FPP and GGPP, which take part in the protein prenylation (Stancu and and Sima, 2001). It was reported that lovastatin treatment cause decreased growth, inhibition of sporangiospore germination and apoptosis-like cell death in Mucor species (Roze and Linz, 1998; Nagy et al., 2014). Transcription of the $h m g R$ genes did not showed significant change in the presence of statins, at the same time overexpression of the $h m g R 2$ and $h m g R 3$ genes led to decreased susceptibility to FLU, atorvastatin and rosuvastatin in M. circinelloides (Nagy et al., 2014). Statins are also able to inhibit the growth of bacteria, although proper mechanisms are not understood. Some studies have attributed the antimicrobial action to the promotion of apoptosis or to the hydrophobic nature of statins causing disruption of the bacterial membrane, resulting in cell death (Bergman et al., 2011; Masadeh et al., 2012).

In our experiments we found that, spatially in case of $m v k$, isoA and $c a r G$ genes, FLU significantly induced the transcription of the genes. Increased accumulation of precursors of isoprenoid-type metabolites assume isoprenoids (such as ergosterol, prenylted proteins and carotenoids) play role in fungal survival in presence of statins. 

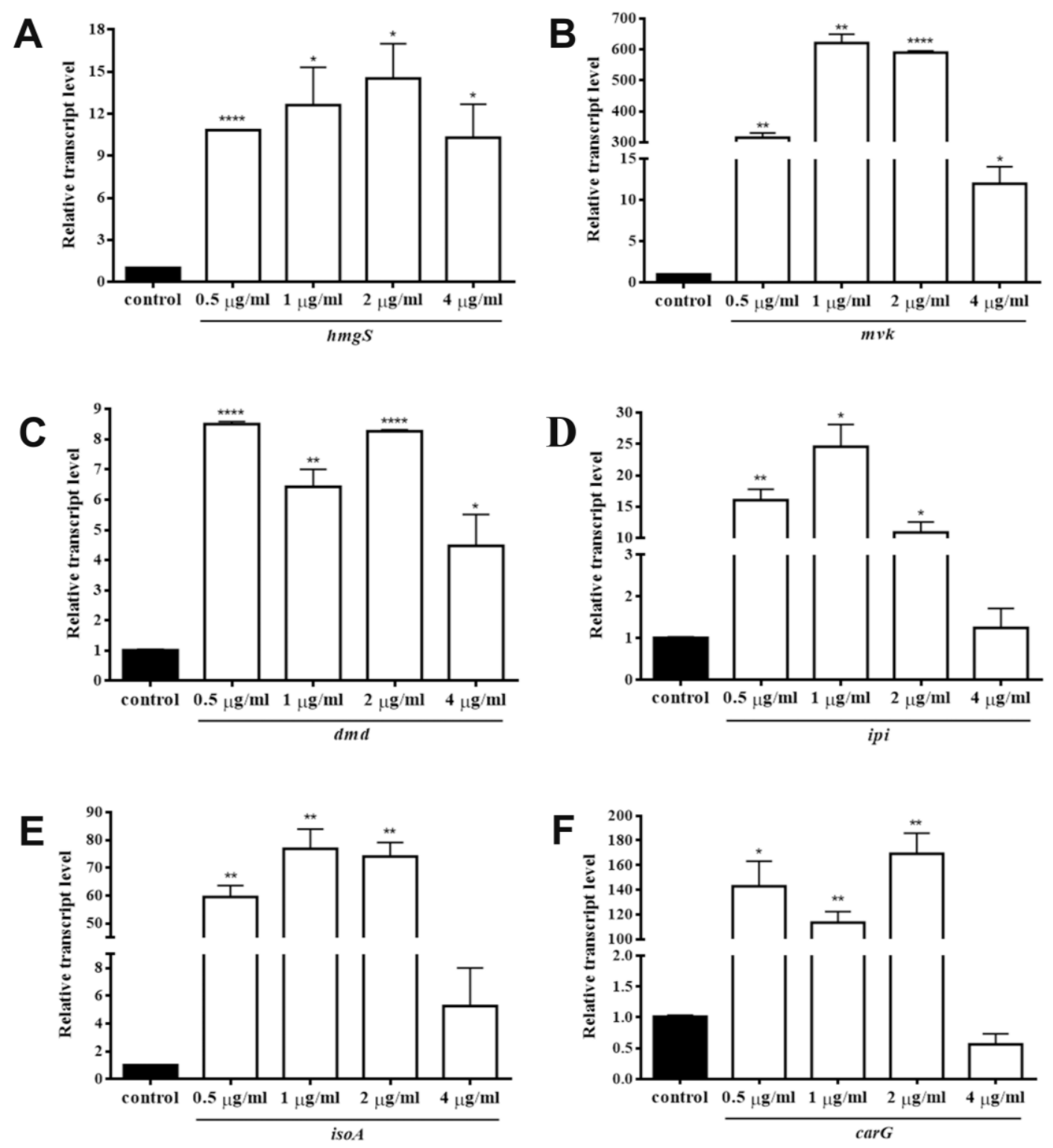

Fig 21. Effect of FLU treatment at different concentrations on the transcription of $M$. circinelloides mevalonate-isoprenoid genes: $h m g S$ (HMG-CoA synthase), $m v k$ (mevalonate kinase), dmd (diphosphomevalonate decarboxylase), ipi (IPP isomerase), isoA (FPP synthase) and carG (GGPP synthase).

\subsection{Construction of plasmids for overexpression and silencing of $M$. circinelloides six} genes involved in the mevalonate-isoprenoid biosynthesis and transformation experiments

Plasmids were constructed for overexpression and silencing of the hmgS, $m v k, d m d$, $i p i$, isoA and carG genes in $M$. circinelloides. No well-developed transformation system was available for targeted gene disruption in $M$. circinelloides when the experiments started (the CRISPR-Cas9 system was developed to M. circinelloides by our research group in 
2017, Nagy et al., 2017), moreover, metabolites synthesized via the mevalonate-isoprenoid pathway play role in several biological processes and disruption of biosynthesis genes could be lethal for the fungus. Overexpression and silencing are commonly used techniques for functional characterization of genes in fungi, also in Mucoromycotina fungi (Moralejo et al., 2002; Kadotani et al., 2003; Lombraña et al., 2004; de Haro et al., 2009; Csernetics et al., 2011; Nicolas et al., 2015; Zhang et al., 2017). The efficiency of overexpression highly depends on the applied promoter. The $M$. circinelloides gpdl promoter is well characterized: it is strongly expressing and can be induced with increased glucose concentration (Wolf and Arnau, 2002), moreover it has been used successfully in several studies (Hirano et al., 2000; Lima et al., 2003; Lopes et al., 2008; Csernetics et al., 2011, 2015, Papp et al., 2013).

For overexpression the genes were amplified together with their own promoter and terminator regions or were placed under the control of the $M$. circinelloides gpdl promoter and terminator (own and gpd constructs, Fig. 8 A-B, Table 3, chapter 5.4. Plasmid constructs used in this study). For gene silencing three different plasmids were constructed for all six genes: (1) the genes were inserted between the Mucor gpdl and zrtl promoter regions ( $P M A T$ constructs, Fig. 8 C, Table 3); (2) a fragment of a gene was inserted between the Mucor gpdl promoter and terminator in inverted orientation (as constructs, Fig. 8 D, Table 3); (3) a fragment of a gene and its reverse complement together with an intron were placed under the regulation of the gpdl promoter and terminator ( $h p R N A$ constructs, Fig. 8 E, Table 3). The Mucor pyrG and leuA genes were used as selection markers, which complement the uracil and the leucine auxotrophy, respectively. $M$. circinelloides MS12 a leuA $A^{-}, \operatorname{pyr}^{-}$mutant derived from the $M$. circinelloides CBS 277.49 was used in all transformation experiments (Benito et al., 1992).

Successful PEG/ $\mathrm{CaCl}_{2}$-mediated transformation of $M$. ciricinelloides protoplasts with the plasmids was carried out. Transformation with pAVB107 and pEPM901 plasmids (harbouring the $l e u A$ and $p y r G$ genes, respectively) was also performed and beside the $M$. circinelloides MS12 those transformants were used as control. The mutants were selected based on auxotrophy complementation on selective YNB medium. The transformation frequency was $1-15$ colony $/ 10^{5}$ protoplasts $/ 5$ - $10 \mu \mathrm{g}$ plasmid DNA and lower number of transformants could have been isolated in case of transformation with the $h p R N A$ plasmids ( 1 - 5 colony/transformation). The presence of plasmid DNA in mutants was analyzed and verified by PCR with primers shown in Table 2 (data not shown). Four transformants were 
inoculated for further investigation from each layout. Examination of the transformants was started after $3-5$ consecutive cultivation cycles on selective YNB medium.

\subsection{Characterization of the mevalonate-isoprenoid biosynthesis pathway mutant $M$. circinelloides strains}

\subsubsection{Examination of plasmid copy number and relative transcription level change in the mutants}

The plasmid copy number was investigated in the mutants with quantitve real-time PCR and found as 0.3 - 10 copy/genom. No difference was observed in copy number between the mutants harbouring different plasmid constructs, but fluctuation was verified within the consecutive cultivation cycles (data not shown); this fluctuation can be due to unequal distribution of the plasmids in spores. Previously, our research group observed similar fluctuation in the plasmid copy number when ipi, isoA and carG genes were overexpressed or the crtS and crtR genes of $X$. dendrorhous were expressed in $M$. circinelloides (Csernetics et al., 2011, 2015);

Next, change in the relative transcription level of the overexpressed and silenced genes were analyzed. We observed an increase in the relative transcription level of the ovexpressed genes that was more prominent in case of the mevalonate-isoprenoid pathway mutants harbouring the genes in extra copies under the control of the gpdl promoter and terminator (Fig. 22). Similar increase was observed in a previous study of our research group, in those transformants harbouring the ipi, isoA and $\operatorname{car} G$ genes under the control of the gpdl regulatory sequences, showed higher transcription level than those with the native regulatory regions (Csernetics et al., 2011). The gpdl promoter was used several times for expression of genes with high efficiency in Rhizomucor miehei (Vastag et al., 2004), $P$. brasiliensis (Barbosa et al., 2004), A. nidulans (Lima et al., 2003) and A. bisporus (Lopes et al., 2008). Silencing with the $h p R N A$ and as plasmid constructs resulted in significant decrease in the relative transcription level in most of the transformants, from which the most prominent decrease was observed in the case of using the hpRNA plasmids (Fig. 22). It has to be mentioned that high deviation in the relative transcription levels was observed in some of the transformants (e.g. in case of MS12+pDMD/hpRNA and MS12+pISOA/hpRNA) which caused by high transcription level detected in one out of four analyzed mutants. In most cases with $p M A T$ plasmid constructs increase was observed 
in the relative transcription levels, thus with as and $h p R N A$ constructs higher silencing efficiency could reach in case of the investigated genes. The variance in the transcription levels could be due to the mitotic instability of the plasmids, thus fluctuation in the plasmid copy number. Moreover, in the case of silencing, a post transcriptional gene silencing was carried out and decrease in the relative transcription cannot be detected necessarily. In $M$. circinelloides a plasmid similar to $h p R N A$ construct of our study was used to silence the carB (encoding phytoene dehydrogenase) gene constructed by inserting an inverted-repeat sequence corresponding to the $c a r B$ gene under the control of the $M$. circinelloides gpdl and own promoter. More albino transformants was isolated using plasmid with gpdl promoter, which could trigger silencing of the $\operatorname{carB}$ gene in over $85 \%$ of the transformants (de Haro et al., 2009). Our aim was also to compare the efficiency of overexpression and silencing using different plasmids in $M$. circinelloides, which may serve valuable information for future studied. On the basis of the results of the present study we are concluding that overexpression and silencing was the most effective using the gpd and $h p R N A$ plasmid constructs, respectively.

The mitotic stability of the transformants was also analyzed with 12 consecutive cultivation cycles on MEA medium, followed by inoculation onto selective YNB medium. Transformants of MS12+pHMG/hpRNA, MS12+pDMD/hpRNA and MS12+pIPI/hpRNA were less stable, which were unable to grow on YNB after $4^{\text {th }}-7^{\text {th }}$ cycle of cultivation on MEA medium. Moreover, overexpression and silencing of the isoA (MS12+pISOA/gpd and MS12+pISOA/as) also resulted reduction in mitotic stability. These results presume, that silencing of the $h m g S, d m d$, ipi (precursors of all isoprenoids) and modification of the isoA (precursor of ergosterol and functional group of farnesylated proteins) have significant effect to the fungus.

Previously it was shown that transformants of $M$. circinelloides and closely related species (i.e. Rhizopus oryzae) carrying autonomously replicating vectors are often unstable and the copy number of the circular plasmid remains low (Anaya and Roncero 1991; Ibrahim and Skory 2006; Mertens et al. 2006). In contrast with these results, we found that $M$. circinelloides transformants are mitotically stable (except MS12+pHMG/hpRNA, MS12+pDMD/hpRNA and MS12+pIPI/hpRNA). In a previous study transformants harbouring plasmid for overexpression of the ipi, isoA and $\operatorname{car} G$ genes were mitotically stable after ten consecutive cultivation cycles on minimal (YNB) and complete (MEA) media (Csernetics et al., 2011). Cultivation on complete medium did not resulted in a decrease in the plasmid copy number, however, fluctuations were observed generally, 
irrespectively of that the transferred gene was homologous or heterologous (Csernetics et al., 2011; 2015).
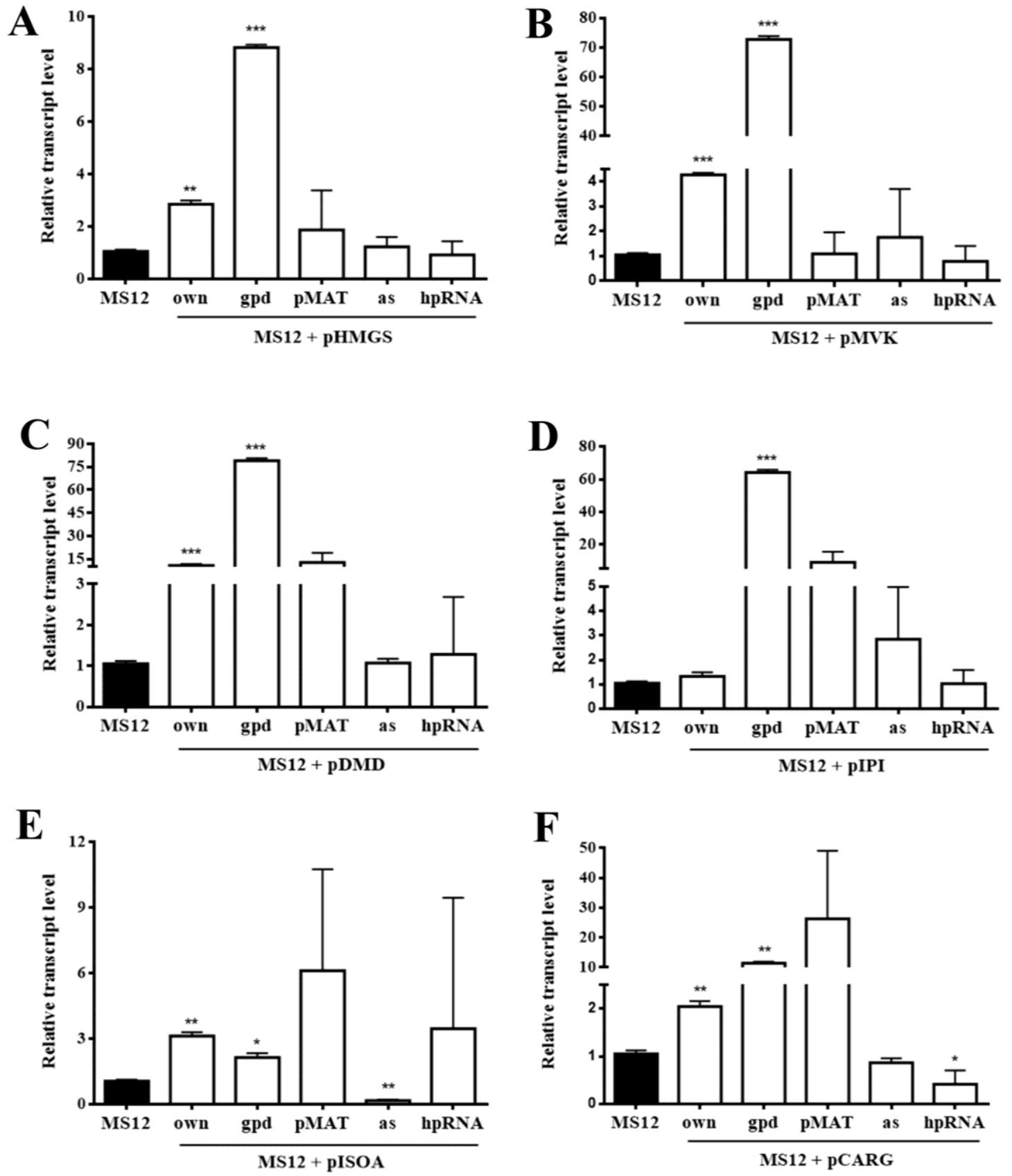

Fig 22. Relative transcription level of transformants harbouring the plasmids for overexpression (own and $g p d$ ) and silencing ( $p M A T$, as and $h p R N A$ ) of the six mevalonate-isoprenoid genes in $M$. circinelloides (plasmid details shown in Table 3 ).

\subsubsection{Investigation of macro- and micromorphology of the transformants}

The colony forming unit (CFU) gives information about the plasmid distribution and viability of spores, which was studied with inoculation of $2 \times 10^{2}$ spores onto selective 
YNB medium ( $\mathrm{pH}$ 3.2). All transformants showed significant reduction in CFU compared to the M. circinelloides MS12 (Fig. 23), also those harbouring the pAVB107 and pEPM901 plasmids (Fig. 23 A). The reduction in CFU could be due to unequal distribution of plasmids and it does not depend on the plasmid sequence or the marker gene. This observation was verified in case of MS12+pAVB107 and MS12+pEPM901, in which the leucine and uracil auxotrophy was complemented and CFU of those transformants also reduced significantly (Fig. 23 A). When transformants were compared with those harbouring plasmids with marker gene only, we found that mutants in which silencing was achieved with hpRNA plasmid (MS12+pHMGS/hpRNA, MS12+pDMD/hpRNA and MS12+pISOA/hpRNA) showed significant reduction in CFU (Fig. 23 B, D and F). It seems that silencing of $h m g S$, dmd and isoA with $h p R N A$ plasmids result in significant decrease in the viability of spores.

Spore germination was investigated with two different incubation time and media: at four hours and eight hours postinoculation in liquid media and on solid media (on cellophane discs); the examination was carried out with bright field microscope. All $M$. circinelloides mutants in the mevalonate-isoprenoid pathway showed significant decrease in number of germinating spores in comparison with $M$. circinelloides MS12. Similar decrease was also observed in MS12+pEPM901 strain harbours the plasmid with only the marker gene (data not shown), which presume that decrease in number of germinating spores was caused mainly by unequal distribution of plasmids and not the modification of the mevalonate-isoprenoid pathway.

The colony diameter of the strains was determined at 24 hours intervals up to four days, with inoculation of $10^{5}$ spores on YNB selective medium (Fig. 24 A-G). No any significant changes were found between M. circinelloides MS12 and MS12+pAVB107 and MS12+pEPM901 strains in colony diameter (Fig. 24 G). In general, silencing with the $h p R N A$ plasmids resulted decrease in the colony diameter in case of all six genes (but not at all intervals) in comparison with the M. circinelloides MS12 (Fig. 24 A-G). It has to be mentioned that in case of MS12+pHMGS/as, MS12+pDMD/as, MS12+pIPI/as and MS12+pCARG/as transformants also significant reduction in colony diameter was observed in comparison with M. circinelloides MS12 (Fig. 24 A-G), presuming that the observed changes was the effect of the silencing of the mevalonate-isoprenoid biosynthesis pathway genes of $M$. circinelloides and not the fluctuation in plasmid copy number. 

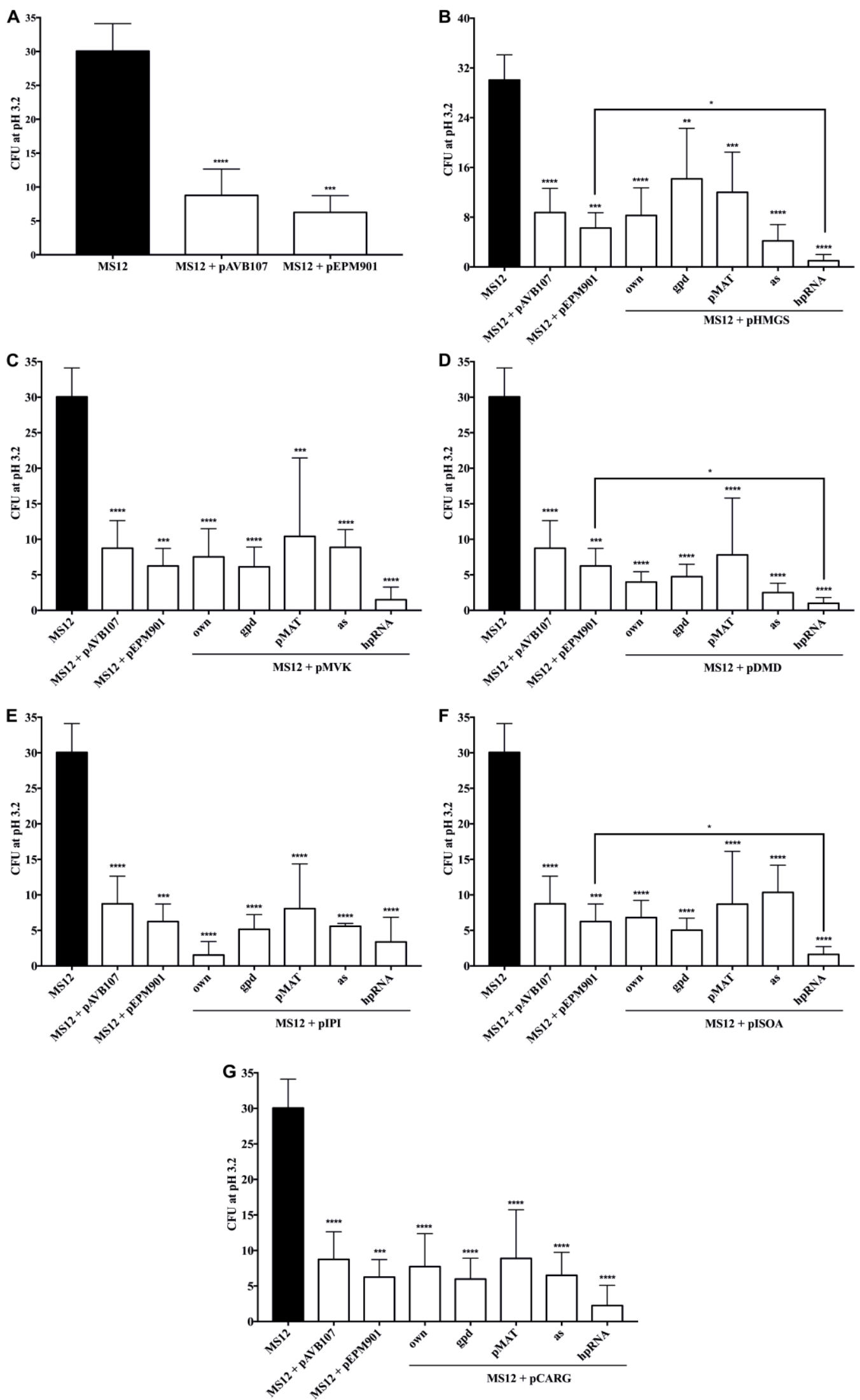

Fig 23. Percentage of colony forming units (CFU) of the $M$. circinelloides MS12, MS12+pAVB107 and MS12+pEPM901 (A) and mutants in the mevalonate-isoprenoid pathway (BG) on YNB selective medium. 

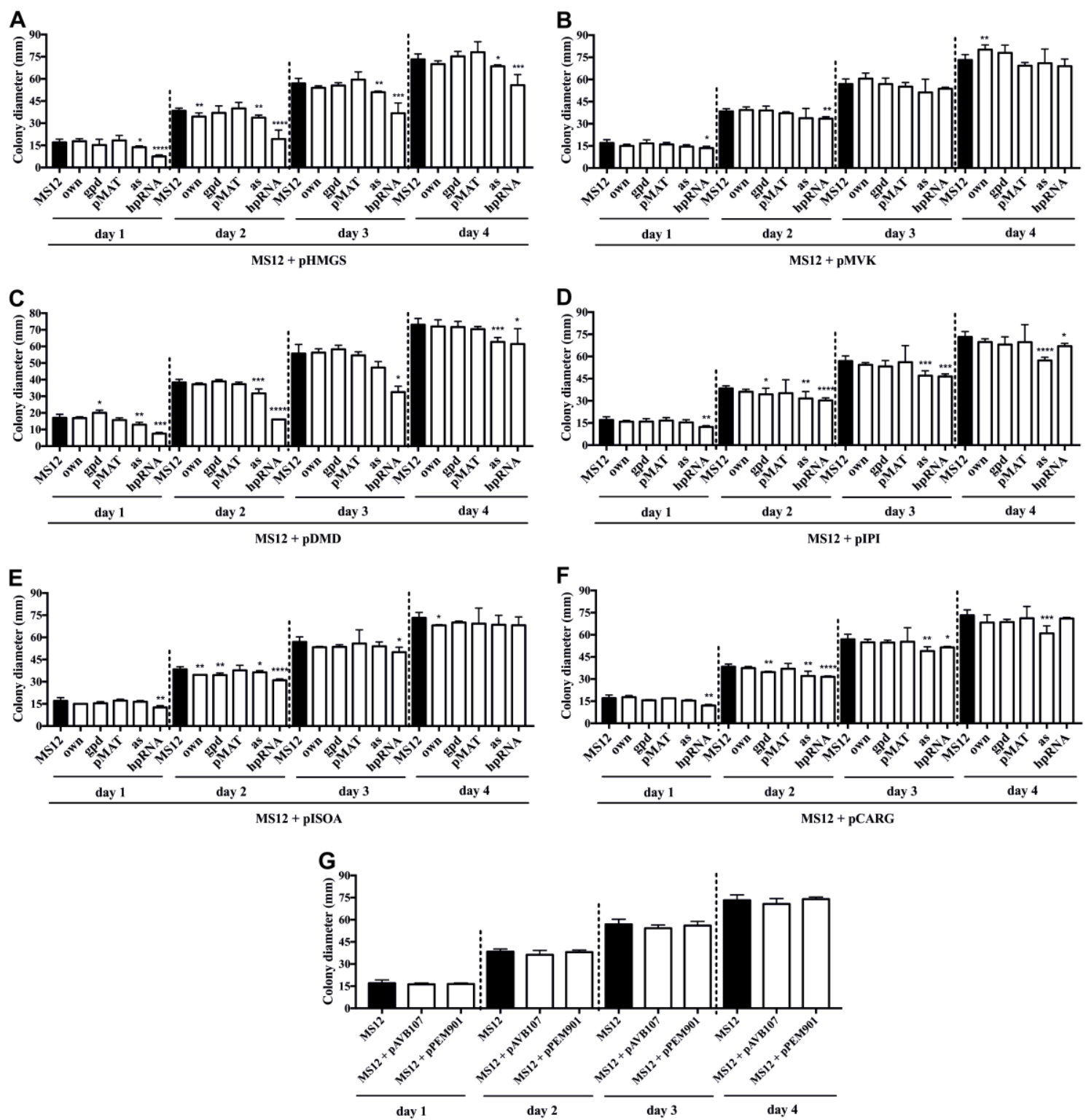

Fig 24. Colony diameter of the $M$. circinelloides strains mutant in mevalonate-isoprenoid pathway (A-F) and MS12+pAVB107 and MS12+pEPM901 strains (G), which was determined at 24 hours intervals up to four days.

Micromorphology of the transformants (including hyphae branching and cytoplasmic effusion) was investigated under bright field microscope (Fig. 25 A-L). We found that MS12+pDMD/hpRNA mutants need more time for the growth. Moreover, in most of the transformants increased number of hyphal branches and cytoplasmic effusion was observed in four days old cultures. To quantify, the strains were inoculated onto cellophane discs after taht hyphal branches and cytoplasmic effusions were counted in 24 hours old cultures. 

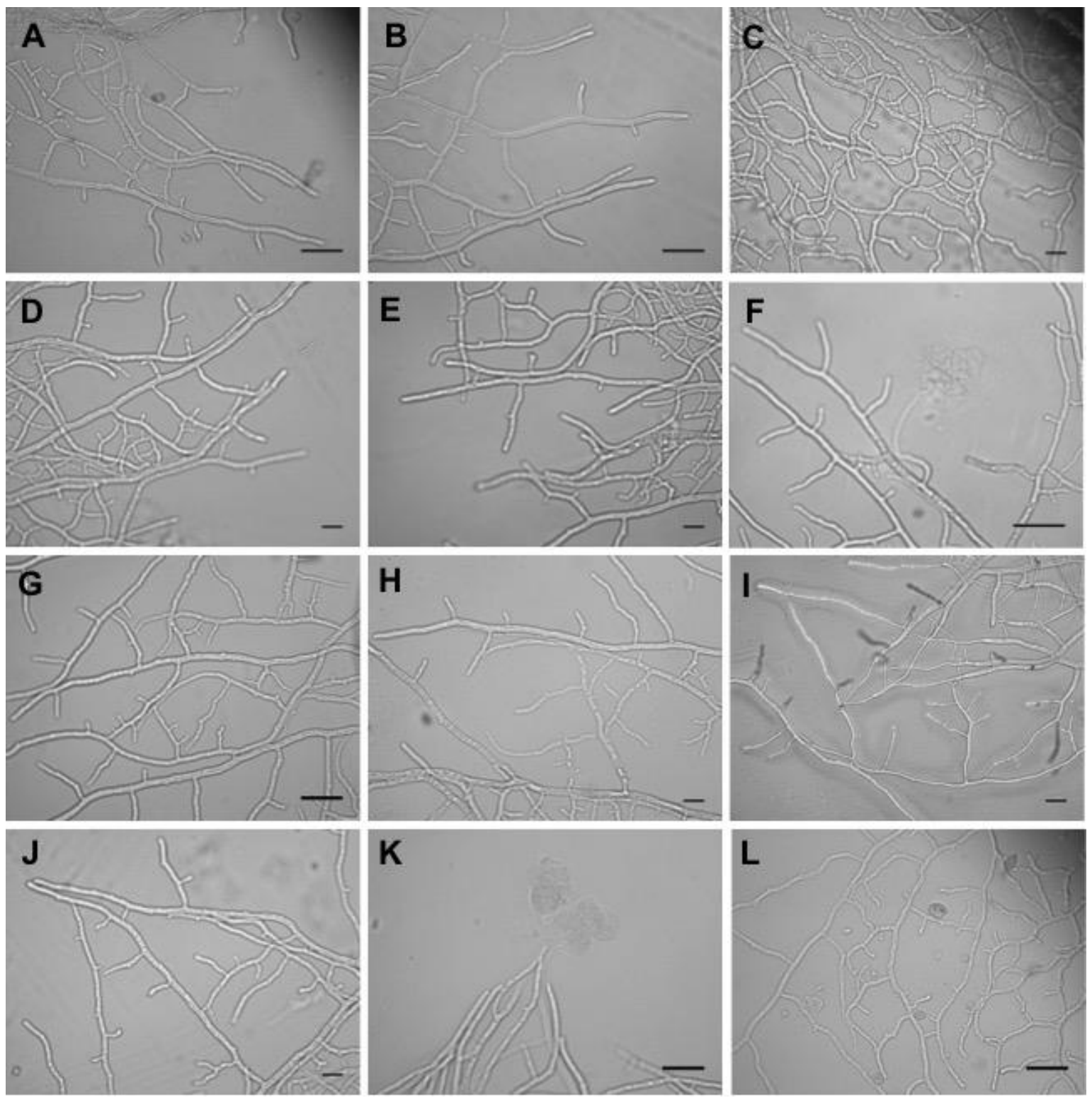

Fig 25. Micromorphology of selected transformants observed under bright field microscope: (A) MS12, (B) MS12+pEPM901, (C) MS12+pHMGS/own, (D) MS12+pMVK/own, (E) MS12+pDMD/AS, (F) MS12+pDMD/hpRNA, (G) MS12+pIPI/gpd, (H) MS12+pISOA/gpd, (I) MS12+pISOA/hpRNA, (J) MS12+pCARG/gpd, (K) MS12+pCARG/as and (L) MS12+pCARG/hpRNA, scale bars for each picture are $100 \mu \mathrm{m}$.

The branches were counted on hyphae originates from one germ tube and we found that mutant strains showed increase in hyphae branching in comparison to $M$. circinelloides MS12 (Fig. 26). In case of MS12+pAVB107 no difference was observed in comparison with $M$. circinelloides MS12, while in number of hyphae branches significant increase was found at MS12+pEPM901 (Fig. 26). It seems that complementation of the uracil auxotrophy has effect on the micromorphology of $M$. circinelloides: it increase the number of hyphae branches, while, interestingly, in case of mutants harbouring plasmids with the leuA marker gene (i.e. MS12+pAVB107, MS12+pISOA/gpd and 
MS12+pCARG/gpd) this phenomenon could not be observed (except MS12+pISOA/own, in which slight increase was observed in number of hyphae branches in comparison with MS12).

A

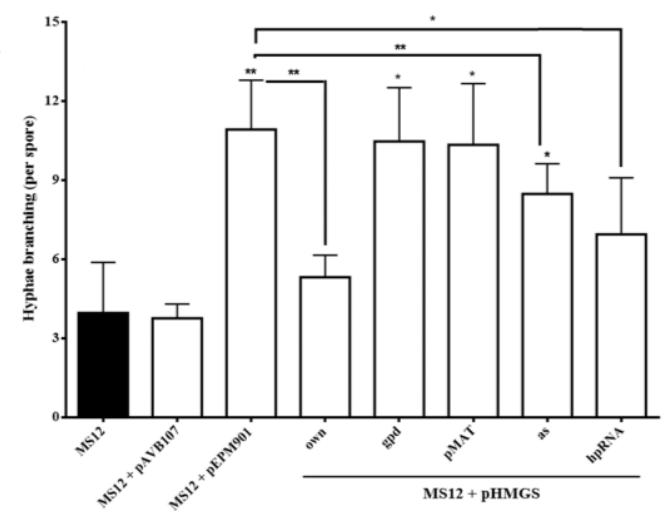

C

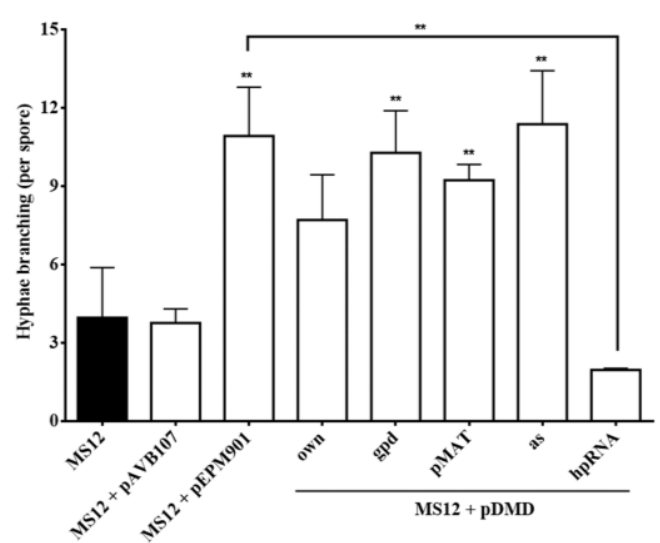

$\mathbf{E}$

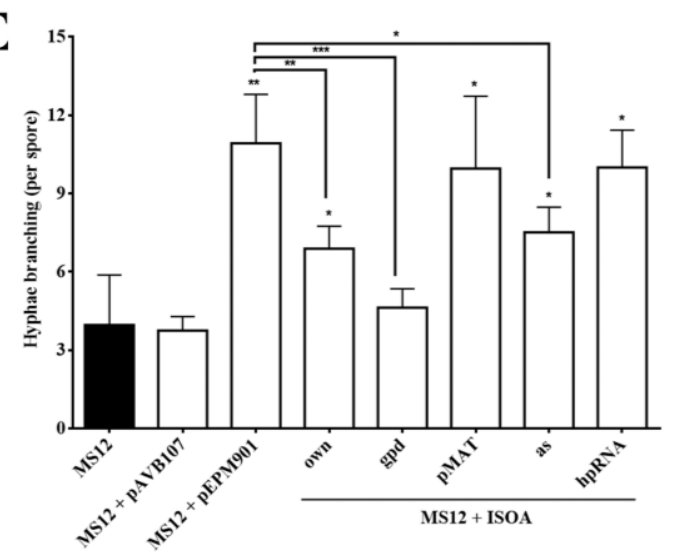

B

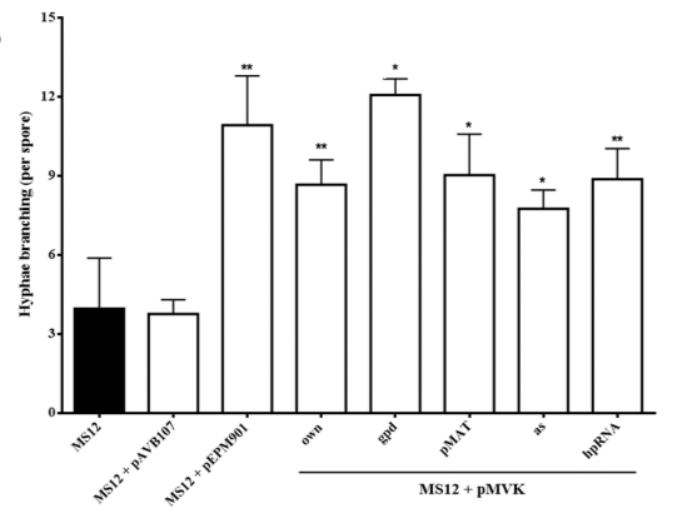

D

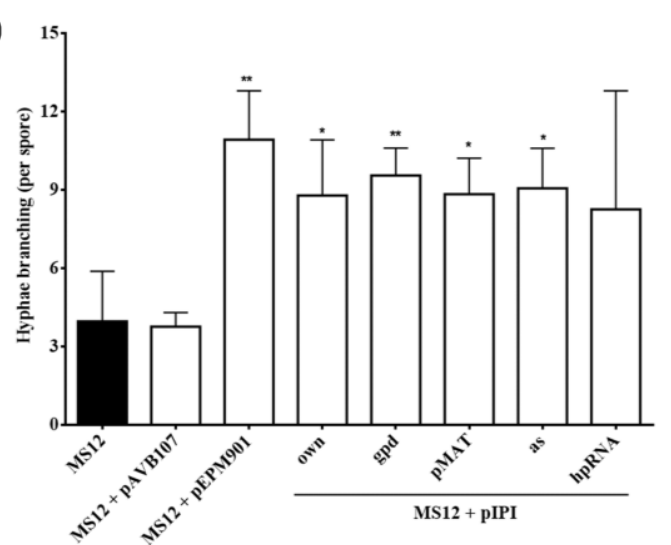

F

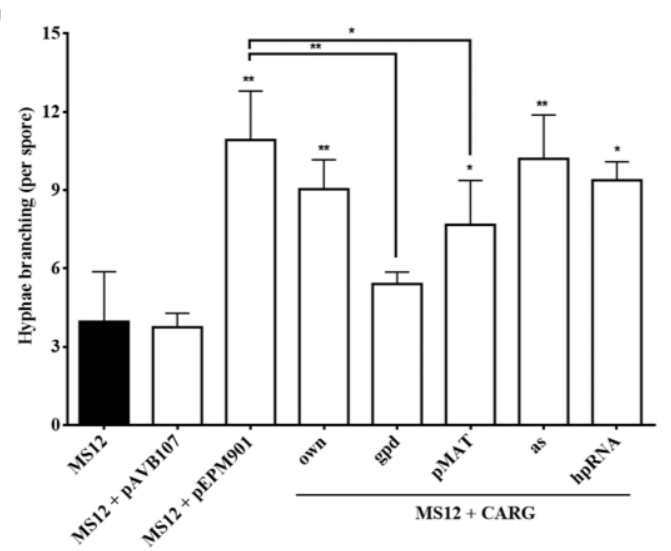

Fig 26. Number of hyphae branching analyzed at per microscopic area of M. circinelloides strains mutant in mevalonate-isoprenoid pathway (data are representing with comparison to $M$. circinelloides MS12 and MS12+pPEPM901). 
The hyphal branching counted in the mutants was also compared to MS12+pEPM901. In this comparison, taking into account the above mentioned observations, mainly overexpression of the $h m g S$ with own plasmid and silencing of the $d m d$ and isoA with $h p R N A$ and as plasmids, respectively, resulted decrease in number of hyphae branching (Fig. 26). It has to be noted that in four days old cultures an increase number in hyphal branching was observed, but in those cultures counting was not performed due to technical difficulties. Together with the results of colony diameter measurments it seems that silencing of the genes, mainly those responsible for formation of IPP $(d m d)$ and precursors of ergosterol and prenyl groups of proteins (isoA) have the major effect in colony growth. IPP is the binding block of all isoprenoids, and FPP is the precursor of ergosterol. Sterol-rich membrane domains define prospective growth sites in fission yeasts (Makushok et al., 2016). Ergosterol is an important component of fungal cell membrane and decrease in the ergosterol content may result in abnormal hyphal branches in filamentous fungi (Lin and Momany, 2004; Alvarez et al., 2007; Abe et al., 2009).

In four days old mycelia increased number of cytoplasmic effusion was observed in comparison with the $M$. circinelloides MS12, which was quantified in 24 hours old mycelia. Ergosterol is playing role in maintenance of fungal membrane intergrity; cytoplasmic effusion presume fungal cell membrane integrity damage, which can be caused by decrease in the ergosterol content (Alvarez et al., 2007; Abe et al., 2009). In case of young mycelia no significant increase was observed in the number of cytoplasmic effusions of the transformants in comparison with $M$. circinelloides MS12 or MS12+pPEPM901 (Fig 27).

Apoptosis is an important process in multicellular organisms (Collins et al., 1992). This normally occurs during the progression of a cell and it is associated with maintenance of cell homeostasis, removal of damaged cells, response to infectious agents, and differentiation, as well as in the acclimatize responses of cells to biotic and abiotic stresses (Danial and Korsmeyer, 2004; Green, 2005). Apoptotic and necrotic cells of mutants harbouring the $h p R N A$ plasmids for gene silencing were quantified after four and eight hours postinoculation. In case of MS12+pMVK/hpRNA, MS12+pDMD/hpRNA, MS12+pIPI/hpRNA and MS12+pCARG/hpRNA significant increase in the number of apoptotic cells was observed after four hours, however significant increment of necrotic cells was quantified only in MS12+pHMGS/hpRNA and MS12+pCARG/hpRNA compared to $M$. circinelloides MS12. While, after eight hours postinoculation, 
significantly increased number of apoptotic cells was observed in the transformants of MS12+pMVK/hpRNA, MS12+pDMD/hpRNA, MS12+pIPI/hpRNA and MS12+pCARG/hpRNA, parallel with necrotic cells were observed in MS12+pHMGS/hpRNA compared to M. circinelloides MS12 (data not shown).
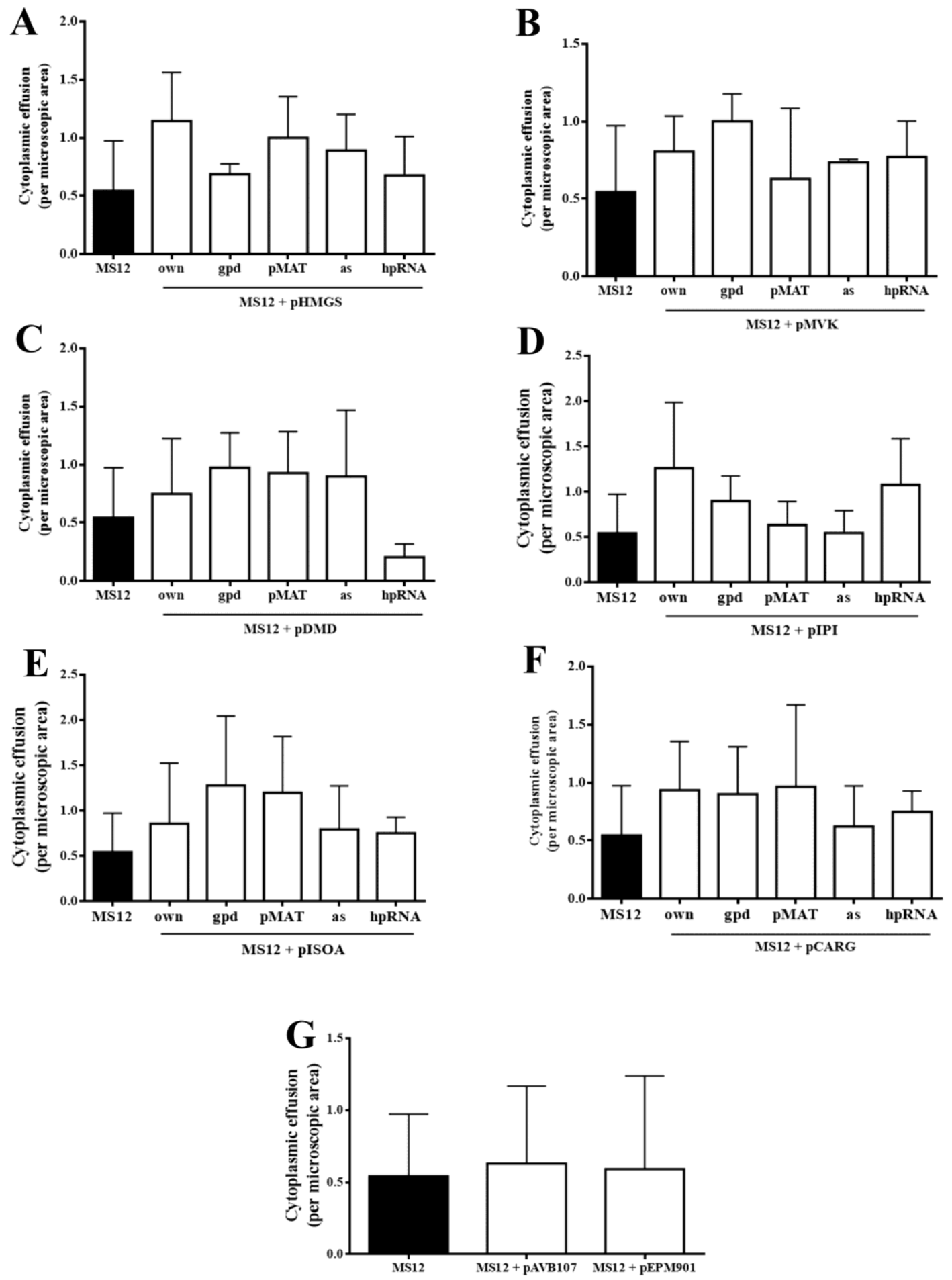

Fig 27. Number of cytoplasmic effusions at per microscopic area in M. circinelloides MS12 mutant of mevalonate-isoprenoid pathway (A-F), MS12 + pAVB107 and MS12 + pEPM901 (G). 


\subsection{Carotenoid and ergosterol content of the $M$. circinelloides strains mutant in mevalonate-isoprenoid pathway}

Carotenoids are secondary metabolites, which are synthesised via the mevalonateisoprenoid pathway in fungi. Carotenoid content of the mevalonate-isoprenoid biosynthesis pathway mutant $M$. circinelloides strains was analyzed. Overexpression of the genes resulted in increase in the carotenoid content, which was most prominent in transformants harbouring the plasmids with genes placed under the control of the gpdl promoter and terminator. The most significant increase was observed in case of overexpressing of the carG, followed by ipi and $m v k$ genes, while silencing of $h m g S$, $m v k$ and $d m d$ (MS12+pHMGS/hpRNA, MS12+pMVK/hpRNA and MS12+pDMD/as) resulted in significant decrease in carotenoid production in comparison with the $M$. circinelloides MS12. Presumably by silencing the early mevalonate-isoprenoid pathway genes the precursors of carotenoid biosynthesis decreased, resulting in less accumulation of carotenoids. Interestingly, silencing of isoA gene resulted slight increase in the carotenoid content in comparison with the $M$. circinelloides MS12, while silencing of the ipi and carG had no any significant effect on the carotenoid production (Fig. 28). In strains MS12+pAVB107 and MS12+pEPM901 no significant difference was found in the carotenoid content in comparison with M. circinelloides MS12 (Fig. 28 G). The carotenoid composition of several mutants was investigated with HPLC and was found that overexpression or silencing of the genes do not affect significantly the carotenoid composition, thus $\beta$-carotene remains the main carotenoid compound and $\gamma$-carotene, lycopene, zeaxanthin and $\beta$-cryptoxanthin production in low amount could be also detected (data not shown).

In our study, overexpression of the mvk resulted significant increase in the carotenoid content. Mevalonate kinase encoded by the $m v k$ gene is responsible for the formation of mevalonate-5P, a middle step in the mevalonate pathway. In comparison of the transcription of the investigated six genes in M. circinelloides, mvk showed the lowest transcription level (Fig. 10). With overexpression of the gene and presumably with increased availability of the precursor resulted significant increase in the accumulation of carotenoids. Overexpression of the ipi and carG genes, similarly to the previous study of our research group, increased the carotenoid production in $M$. circinelloides, moreover the increment was more prominent in case when the genes were placed under the control of the gpdl promoter (Csernetics et al., 2011). For example, in that study three times higher 
carotenoid content than that of the original strain was found when expression of the ipi gene was driven by the gpdl promoter (Csernetics et al., 2011). In our study less difference was observed, i.e. overexpression of the ipi gene with own promoter resulted $646 \mu \mathrm{g} / \mathrm{g}$ dry weight carotenoid content, while in case when the gene was placed under the control of the $g p d l$ promoter resulted $920 \mu \mathrm{g} / \mathrm{g}$ dry weight in compare to the control $M$. circinelloides MS12, $426 \mu \mathrm{g} / \mathrm{g}$ dry weight (in Csernetics and coworkers study it was 674 $\mu \mathrm{g} / \mathrm{g}$ dry weight and $1177 \mu \mathrm{g} / \mathrm{g}$ dry weight, in compare to the control $M$. circinelloides MS12, $399 \mu \mathrm{g} / \mathrm{g}$ dry weight respectively, Csernetics et al., 2011). This variation in the carotenoid production between the two studies can be due to differences in the plasmid copy numbers. Overexpression of the $\operatorname{car} G$ resulted the most significant increase in the carotenoid production of $M$. circinelloides in both studies. In our study when the expression of $\operatorname{carG}$ was driven by its own promoter, $665 \mu \mathrm{g} / \mathrm{g}$ dry weight carotenoid content was determined, while in the case of $g p d l$ promoter $1045 \mu \mathrm{g} / \mathrm{g}$ dry weight. All these findings demonstrate that formation of mevalonate-5P, DMAPP and GGPP are a rate-limiting steps in the carotenoid biosynthesis in $M$. circinelloides.

Beta-carotene accumulation was achieved by heterologous expression of the $X$. dendrorhous phytoene desaturase and phytoene synthase/lycopene cyclase encoding genes in the non-carotenoid producing $S$. cerevisiae and it was enhanced by the overexpression of the S. cerevisiae BTS1 gene, encoding the geranylgeranyl pyrophosphate synthase (Ukibe et al., 2009). The intermediate enzyme of mevalonate-isoprenoid biosynthesis pathway, such as IPP isomerase, has been presumed to be a key regulatory enzyme in the isoprenoid pathway, and the effect of its (over) expression on carotenoid biosynthesis has been studied in detail in carotenoid producing mutant E. coli (Lee and Schmidt-Dannert, 2002; Das et al., 2007). E. coli engineered with heterologous cDNAs of IPP isomerases from Haematococcus pluvialis and $X$. dendrorhous resulted enhanced carotenoid accumulation (Misawa and Shimada, 1997; Albrecht et al., 1999). Indeed, the introduction of Enterococcus faecalis mvaE and mvaS genes, encoding the acetoacetylCoA synthase/HMG-CoA reductase and HMG-CoA synthase, respectively, into E. coli has been reported to improved direct production of mevalonate and substantially improve the productivity of carotenoids or sesquiterpenes that are synthesized from DMAPP (Middleton, 1972; Tabata et al., 2004; Anthony et al., 2009; Yang et al., 2012). In $N$. crassa increased accumulation of the HMG-CoA, led to a several-fold improvement in lycopene and neurosporaxanthin production (Wang and Keasling 2002). Overexpression of the $h m g S$, responsible for the formation of HMG-CoA in M. circinelloides, also led to a 
significant increase in the carotenoid accumulation in our study. Overexpression of genes involved in the early steps in isoprenoid biosynthesis pathway, such as the $h m g R 2$ and $h m g R 3$ genes also led to carotenoid overaccumulation in M. circinelloides (Nagy et al., 2014).
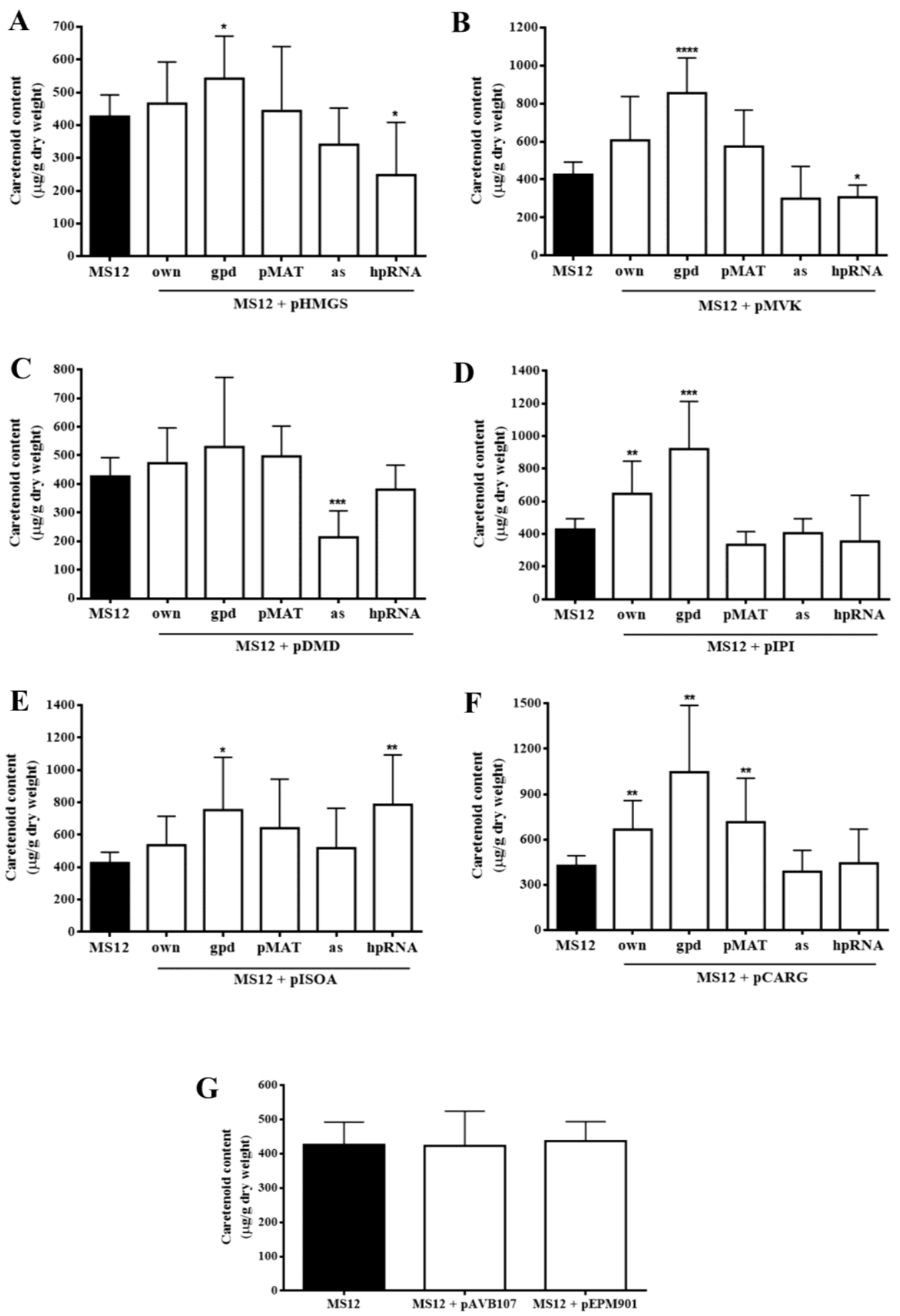

Fig 28. Carotenoid content of the M. circinelloides mutants in mevalonate-isoprenoid biosynthesis pathway. Data represent the carotenoid content in $\mu \mathrm{g} / \mathrm{g}$ dry weight of mycelia. 
Today the Mucoromycotina B. trispora is still used for industrial $\beta$-carotene production. It has to be mentioned that the total carotenoid content of the wild-type $B$. trispora is $260-300 \mu \mathrm{g} / \mathrm{g}$ dry weight, which is similar to M. circinelloides MS12 (Mehta et al., 2003). For industrial carotenoid production mixed culture of different mating types of B. trispora and optimized fermentation media are used (Mehta et al., 2003). However, transformation systems are not available for genetic modification to improve carotenoid production in B. trispora, at the same time efficient transformation systems are available to M. circinelloides and with optimization of the fermentation conditions its carotenoid production could be increased more.

Ergosterol content of the $M$. circinelloides strains mutant in the mevalonateisoprenoid pathway was also analyzed by HPLC. Interestingly, overexpression of the genes did not resulted in significant increment in ergosterol content, however overexpression of the $m v k$, ipi and isoA decreased the ergosterol content. Silencing of all six genes with $h p R N A$ plasmids significantly decreased the content of ergosterol in comparison to the untransformed $M$. circinelloides MS12. No any significant change in the ergosterol content was found between MS12, MS12+pAVB107 and MS12+pEPM901 (Fig. 29).

Nagy and coworkers reported that overexpression of HMG-CoA reductase encoding genes in $M$. circinelloides slightly, but not significant increased the ergosterol content in comparison to the untransformed M. circinelloides MS12 (Nagy et al., 2014). Our results are similar to those, overexpression of the six mevalonate-isoprenoid pathway genes in $M$. circinelloides did not resulted significant ergosterol overaccumulation. At the same time Yasmin and coworkers described that overexpression of hmgl gene, encoding a HMGCoA reducatse increased the ergosterol content in A. fumigatus (Yasmin et al., 2011). In Phaffia rhodozyma it had been shown that astaxanthin overproduction reduced the ergosterol content (leading to precursor accumulation, and transfer to the astaxanthin pathway) compared with the wild type (Miao et al., 2011). Similarly, in our study overexpression of the $m v k$, ipi and isoA genes, as well as silencing of the isoA led to increase in the carotenoid production and reduction in ergosterol accumulation. In case of our study knock down of all six genes by $h p R N A$ plasmids resulted significant reduction in ergosterol content in M. circinelloides with range $40-54 \%$ (Fig. 29). 

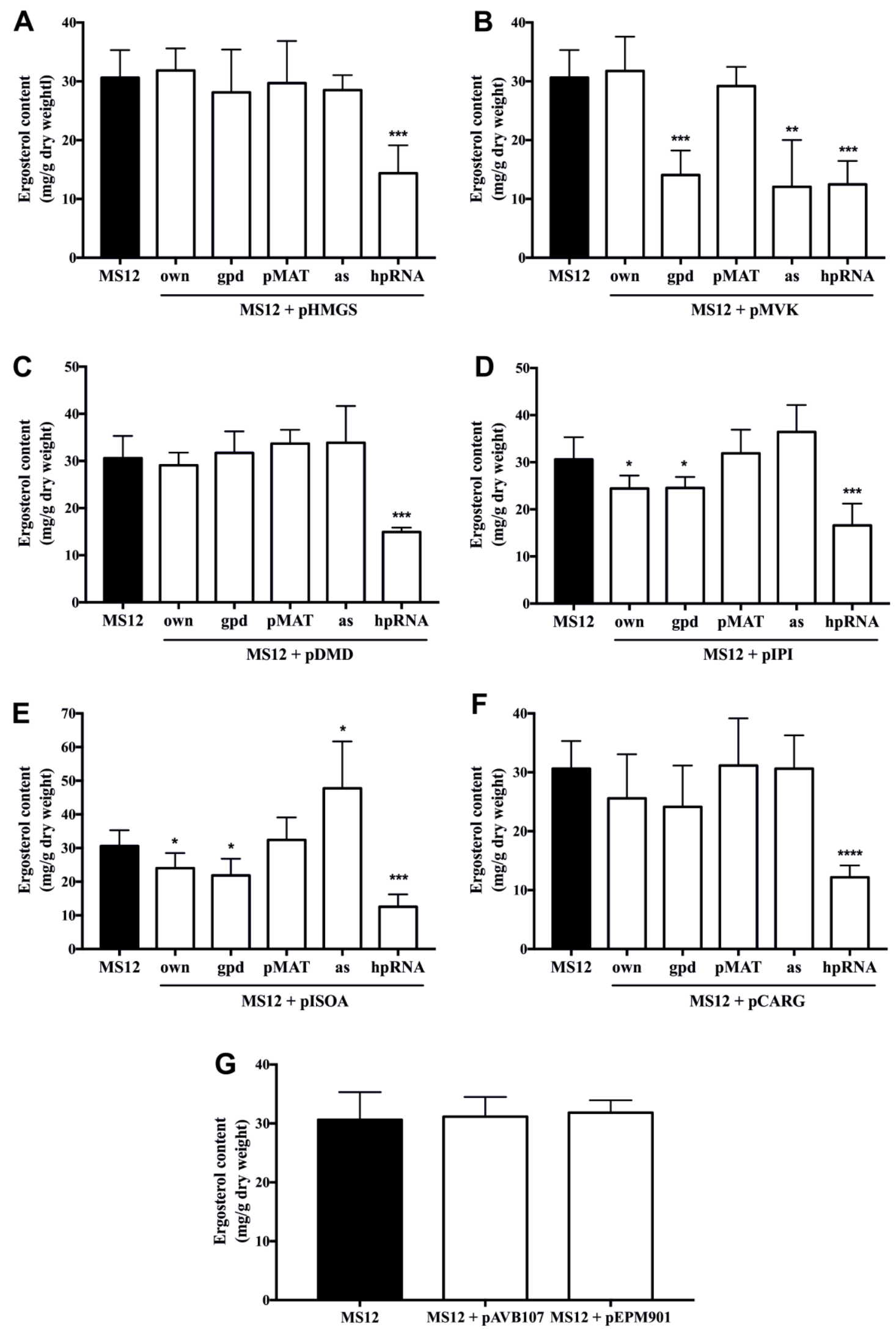

Fig 29. Ergosterol content of the M. circinelloides mutants in mevalonate-isoprenoid biosynthesis pathway, analyzed by HPLC. Data are representing the ergosterol content in $\mathrm{mg} / \mathrm{g}$ dry weight of mycelia. 


\subsection{Antibiotic susceptibility of the $M$. circinelloides strains mutant in mevalonate- isoprenoid pathway}

The mortality rate associated with mucormycoses remains extremely high, while Mucoromycotina fungi shows intrinsic resistance to most of the clinically used antifungal agents, thus identification of new potential novel drug targets would be necessary (Arendrup, 2011; Howard and Arendrup, 2011; van derLinden et al., 2011). Although Mucorales fungi have long been considered a homogenous group in terms of susceptibility to antifungals, it is now clear that this trait differs between species of this order (Alastruey-Izquierdo et al., 2009). Our laboratoary previously analyzed the susceptibility of $M$. circinelloides MS12 against twenty-one antifungals (unpublished data). Based on these data ITR, CLO, SIM, FLU, and AmB were selected for for investigation of the susceptibility of $M$. circinelloides mutants in the mevalonate isoprenoid pathway.

In our study in vitro susceptibility of the mutants in mevalonate-isoprenoid biosynthesis to ITR, CLO, SIM, FLU, and AmB was determined. No any major difference in the susceptibility to $\mathrm{AmB}$ of the mutants was found in comparison with parental strain M. circinelloides MS12. Susceptibility of MS12+pIPI/own, MS12+pHMG/hpRNA and MS12+pDMD/hpRNA mutants significantly changed to ITR, the $\mathrm{MIC}_{90}$ in those strains was determined in $2-8 \mu \mathrm{g} / \mathrm{ml}\left(\mathrm{MIC}_{90}>128 \mu \mathrm{g} / \mathrm{ml}\right.$ in the case of $M$. circinelloides MS12 as well as MS12+pAVB107, MS12+pEPM901), moreover paradox effect was also observed at these strains, thus increasing the ITR concentration to more than $8 \mu \mathrm{g} / \mathrm{ml}$ did not resulted 90\% growth inhibition. This phenomenon was also observed in Candida and Aspergillus species against echinocandins, but the mechanism still not clear, presumably induction of chitin synthesis is one of the possible mechanism of survival (Eagle and Musselman, 1948; Shields et al., 2011; Stover and Cleary, 2015). Increased susceptibility of these strains to CLO was also observed: MS12+pIPI/own, MS12+pHMG/hpRNA and MS12+pDMD/hpRNA showed susceptibility at $1-4,4-16$ and $2-8 \mu \mathrm{g} / \mathrm{ml}$ to CLO, respectively, in comparision to the $32 \mu \mathrm{g} / \mathrm{ml}$ in the case of $M$. circinelloides MS12. It has to be also mentioned that in case of MS12+pEPM901 (4 - 16 $\mu \mathrm{g} / \mathrm{ml}$ ) increased susceptibility to CLO was also determined, but not so prominent as in case of MS12+pIPI/own, MS12+pHMG/hpRNA and MS12+pDMD/hpRNA mutants. Difference in the susceptibility to FLU and SIM was mainly observed in those mutants, in which $m v k$, dmd and ipi were overexpressed or silenced (Table 4). In a previous study, overexpression of the $h m g R 2$ and $h m g R 3$ in M. circinelloides resulted decrease in the susceptibility to fluvastatin, atorvastatin and rosuvastatin (Nagy et al., 2014). Similarly, 
we also observed significant decrease in the susceptibility to FLU and SIM in the case of overexpression of the $m v k$ and $d m d$ genes.

Table 4. Antifungal susceptibility of the mutants in mevalonate-isoprenoid biosynthesis to AmB (amphotericin B), ITR (itraconazole), CLO (clotrimazole), FLU (fluvastatin) and SIM (simvastatin) in vitro.

\begin{tabular}{|c|c|c|c|c|c|}
\hline \multirow{2}{*}{ Strain } & \multicolumn{5}{|c|}{$\mathrm{MIC}_{90}(\mu \mathrm{g} / \mathrm{ml})$} \\
\hline & AmB & ITR & CLO & FLU & SIM \\
\hline MS12 & 1 & $>128$ & 32 & 1 & 1 \\
\hline MS12 + pHMGS-OE & $0.5-2$ & $>128$ & 32 & $1-2$ & $0.5-2$ \\
\hline MS12 + pMVK-OE & $0.5-1$ & $>128$ & 32 & $1-4$ & $1-4$ \\
\hline $\mathrm{MS} 12+\mathrm{pDMD}-\mathrm{OE}$ & $1-2$ & $>128$ & $32->32$ & $2-8$ & $1-4$ \\
\hline $\mathrm{MS} 12+$ pIPI-OE & $0.5-2$ & $2-8 \mu \mathrm{g} / \mathrm{ml}$, paradadox effect & $1-4$ & $0.5-1$ & $0.25-1$ \\
\hline $\mathrm{MS} 12+$ pISOA-OE & $1-2$ & $>128$ & 32 & $0.5-2$ & 2 \\
\hline MS12 + pCARG-OE & $0.5-1$ & $>128$ & 32 & $0.5-2$ & $0.5-4$ \\
\hline MS12 + pHMGS-GPD & 0.5 & $>128$ & $8-16$ & $1-4$ & $0.5-4$ \\
\hline MS12 + pMVK-GPD & 1 & $>128$ & 32 & $4-8$ & $2-8$ \\
\hline MS12 + pDMD-GPD & $0.5-2$ & $>128$ & 32 & $2-8$ & $2-8$ \\
\hline MS12 + pIPI-GPD & $1-2$ & $>128$ & $16-32$ & 1 & $0.5-1$ \\
\hline MS12 + pISOA-GPD & 1 & $>128$ & 16 & 2 & 2 \\
\hline $\mathrm{MS} 12+$ pCARG-GPD & 2 & $>128$ & $16-32$ & $1-2$ & $1-2$ \\
\hline MS12 + pHMGS-AS & 1 & $>128$ & $16-32$ & $0.5-1$ & $0.5-1$ \\
\hline MS12 + pMVK-AS & 0.5 & $>128$ & 16 & $1-2$ & $1-4$ \\
\hline MS12 + pDMD-AS & $0.5-2$ & $>128$ & $8-32$ & $0.5-2$ & $0.125-1$ \\
\hline MS12 + pIPI-AS & $1-2$ & $>128$ & $8-16$ & $0.5-2$ & 1 \\
\hline MS12 + pISOA-AS & 1 & $>128$ & 32 & $0.5-1$ & $0.5-1$ \\
\hline MS12 + pCARG-AS & 2 & $>128$ & $16-32$ & $1-2$ & $1-2$ \\
\hline MS12 + pHMGS-MAT & 0.5 & $>128$ & 16 & 0.5 & 1 \\
\hline MS12 + pMVK-MAT & $0.5-1$ & $>128$ & 32 & 1 & $1-4$ \\
\hline MS12 + pDMD-MAT & 0.5 & $>128$ & 32 & $0.5-1$ & $0.5-2$ \\
\hline MS12 + pIPI-MAT & 2 & $>128$ & 32 & 1 & $2-4$ \\
\hline MS12 + pISOA-MAT & $0.5-2$ & $>128$ & 32 & 1 & $0.5-1$ \\
\hline MS12 + pCARG-MAT & 0.5 & $>128$ & $4-32$ & $0.5-1$ & $0,5-8$ \\
\hline MS12 + pHMGS-HPRNA & $0.5-2$ & $4-8 \mu \mathrm{g} / \mathrm{ml}$, paradadox effect & $4-16$ & $0.5-2$ & $0.5-2$ \\
\hline MS12 + pMVK-HPRNA & $0.5-1$ & $>128$ & $8-16$ & $1-2$ & $0.5-1$ \\
\hline MS12 + pDMD-HPRNA & $0.5-4$ & $2-8 \mu \mathrm{g} / \mathrm{ml}$, paradadox effect & $2-8$ & $0,125-1$ & $0.5-1$ \\
\hline MS12 + pIPI-HPRNA & 1 & $>128$ & $8-16$ & $0.5-4$ & $1-8$ \\
\hline MS12 + pISOA-HPRNA & 1 & $>128$ & 8 & $1-2$ & 1 \\
\hline MS12 + pCARG-HPRNA & $0.5-1$ & $>128$ & 16 & $0.5-2$ & $0.5-1$ \\
\hline MS12 + pAVB107 & 1 & $>128$ & 32 & $1-2$ & 2 \\
\hline MS12 + pEPM901 & $0.5-2$ & $>128$ & $8-16$ & $0.5-1$ & 1 \\
\hline
\end{tabular}

The MIC of AmB $(0.06-1 \mu \mathrm{g} / \mathrm{ml})$, ITR $(2->8 \mu \mathrm{g} / \mathrm{ml})$ and FLU $(>25 \mu \mathrm{g} / \mathrm{ml})$ to some $M$. circinelloides strains was determined in previous studies (Caramalho et al., 2015; Almyroudis et al., 2007; Drogari-Apiranthitou et al., 2012; Salas et al., 2012 Galgóczy et al., 2011). In our experiments $\mathrm{MIC}_{90}$ of $\mathrm{AmB}$ and FLU to MS12 was determined in $1 \mu \mathrm{g} / \mathrm{ml}$, while in case of ITR no MIC 90 could be determined in the investigated concentrations. It has to be mentioned that in our experiments the antifungal susceptibility tests were performed in YNB medium to maintain the selective conditions 
and not in RPMI-1640 as recommended by the CLSI M38-A2 method. Differences in the susceptibility of $M$. circinelloides MS12 to antifungal agents in YNB and RPMI-1640 media was also observed by our research group previously (unpublished data).

Azoles are inhibitors of the cytochrome-P450 dependent lanosterol 14- $\alpha$ demethylase, a key enzyme in ergosterol biosynthesis (Vandeputte et al., 2012). In our experiments overexpression of the ipi and silencing of the $h m g S$ and $d m d$ genes resulted increase in the susceptibility to azoles and these mutants showed reduction in ergosterol content, as well (Fig. 29). Moreover silencing of the $d m d$ gene increased the susceptibility of $M$. circinelloides to statins, which are competitive inhibitors of the HMG-CoA reductase, Nagy et al., 2014). Based on all of these observations, silencing of the $d m d$ gene resulted the most prominent change in the susceptibility to azoles and statins (Table 4).

\subsection{Interaction of the mevalonate-isoprenoid pathway mutant $M$. circinelloides strains with MH-S macrophages}

In our study phagocytosis assay was performed with MH-S macrophages and the mevalonate-isoprenoid pathway mutant $M$. circinelloides strains. Overexpression of the $h m g S$, ipi and $c a r G$ and silencing of $m v k$ and isoA genes (with $h p R N A$ plasmids) resulted significant decrease in the phagocytic indexes (PI), while overexpression of $m v k$ and $d m d$, as well as silencing of $h m g S, m v k, d m d$ and $c a r G$ genes with $p M A T$ plasmid and $m v k$ with as plasmid resulted significant increase in PI in comparison with M. circinelloides MS12 (Fig. 30). In case of MS12+pAVB107 and MS12+pEPM901 no significant change in PI was observed in comparison with $M$. circinelloides MS12 (Fig. 30). Up to days no any data available regarding interaction of macrophages with Mucormycotina fungi mutant in mevalonate-isoprenoids pathway.

Heuston and coworker summarized that classical mevalonate pathway or the alternative 2C-methyl-D-erythritol-4-phosphate (MEP) pathway or its intermediate 1hydroxy-2-methyl-2-(E)-butenyl-4-diphosphate (HMB-PP) pathway can activate human $\mathrm{Vc} 9 / \mathrm{Vd} 2 \mathrm{~T}$-cells (Heuston et al., 2012). Begley and coworkers have previously described that both pathways, either the classical mevalonate pathway or the alternative nonmevalonate are functional in Listeria monocytogenes and can provide sufficient IPP for normal growth (Begley et al., 2004). Murine studies revealed that mutants lacking the MEP pathway were impaired in virulence relative to the parental strain (Begley et al., 2008). Presumably, modification of the amount of accumulated metabolites synthesized 
via the mevalonate-isoprenoid pathway in $M$. circinelloides (such as ergosterol) resulted change in the PI in comparison with the untransformed $M$. circinelloides MS12, however, to uncover the backround further investigations are required.
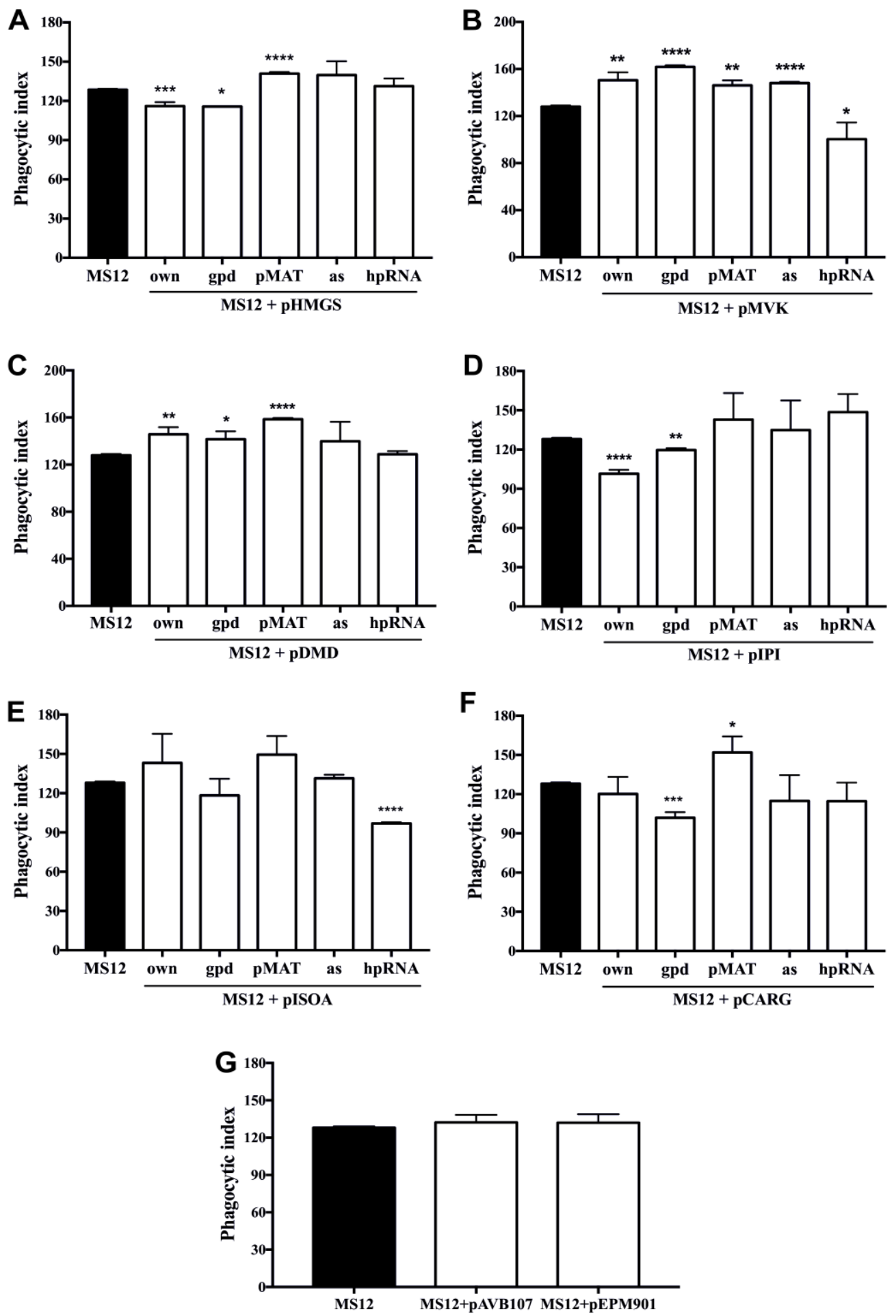

Fig 30. Phagocytic indexes of $M$. circinelloides mutants defective in mevalonate-isoprenoid biosynthesis pathway. 


\section{CONCLUSIONS}

As a result of our research, new information was added to our knowledge regarding the mevalonate-isoprenoid biosynthesis pathway in M. circinelliodes. Six genes involved in that pathway ( $h m g S, m v k, d m d$, ipi, isoA and $c a r G)$ were selected for characterization and the transcription of the genes was determined under different cultivation conditions, which can contribute in further studies to modify the metabolite production of the fungus (such as carotenoids and ergosterol). Characteristics of the mutant $M$. circinelloides strains were also compared to each other and found that overexpression and silencing are the most effective with plasmids harbouring the genes under the control of the gpdl promoter and $h p R N A$ constructs, respectively.

Carotenoid overproducing mutants were isolated with overexpression of the $m v k$, ipi and carG genes, which can be used in further studies to improve the $\beta$-carotenoid production of Mucoromycotina fungi. Moreover, $40-54 \%$ decrease in the ergosterol content was detected with silencing of all six genes with $h p R N A$ plasmid and increased susceptibility to azoles and statins of mutants harbouring the $h p R N A$ plasmid for silencing of $d m d$ gene was determined, which may serve as potential target for new antifungal therapy in future. 


\section{REFERENCES}

Abe A, Sujaya IN, Sone T, Asano K, Oda Y (2004) Microflora and selected metabolites of potato pulp fermented with an Indonesian starter Ragi Tapé. Food Technol Biotechnol 42:169-173.

Abe, F, Usui K, Hiraki T (2009) Fluconazole modulates membrane rigidity, heterogeneity, and water penetration into the plasma membrane in Saccharomyces cerevisiae. Biochemistry 48:8494-8504.

Alastruey-Izquierdo A, Castelli MV, Cuesta I, Zaragoza O, Monzon A, Mellado E, Rodríguez-Tudela JL (2009) In vitro activity of antifungals against zygomycetes. Clin Microbiol Infect 15:71-76.

Albrecht M, Misawa N, Sandmann G (1999) Metabolic engineering of the terpenoid biosynthetic pathway of Escherichia coli for production of the carotenoids B-carotene and zeaxanthin. Biotechnol Lett 21:791-795.

Alcaíno J, Bravo N, Córdova P, Marcoleta AE, Contreras G, Barahona S, Sepúlveda D, Fernández-Lobato M, Baeza M, Cifuentes V (2016) The involvement of Mig1 from Xanthophyllomyces dendrorhous in catabolic repression: an active mechanism contributing to the regulation of carotenoid production. PLoS One 11:e0162838.

Alcazar-Fuoli L, Mellado E, Garcia-Effron G, Lopez JF, Grimalt JO, CuencaEstrella JM, Rodriguez-Tudela JL (2008) Ergosterol biosynthesis pathway in Aspergillus fumigatus. Steroids 73:339-347.

Almyroudis NG, Sutton DA, Fothergill AW, Rinaldi MG, Kusne S (2007) In vitro susceptibilities of 217 clinical isolates of zygomycetes to conventional and new antifungal agents. Antimicrob Agents Chemother 51:2587-90.

Alvarez E, Sutton DA, Cano J, Fothergill AW, Stchigel A, Rinaldi MG, Guarro J (2009) Spectrum of zygomycete species identified from clinically significant specimens in the United States. J Clin Microbiol 47:1650-1656.

Alvarez FJ, Douglas LM, Konopka JB (2007) Sterol rich plasma membrane domains in fungi. Eukaryot Cell 6:755-763.

Amey RC, Athey-Pollard A, Burns C, Mills PR, Bailey A, Foster GD (2002) PEGmediated and Agrobacterium-mediated transformation in the mycopathogen Verticillium fungicola. Mycol Res 106:4-11. 
Anaya N, Roncero MI (1991) Transformation of a methionine auxotrophic mutant of Mucor circinelloides by direct cloning of the corresponding wild type gene. Mol Gen Genet 230:449-455.

Anthony JR, Anthony LC, Nowroozi F, Kwon G, Newman JD, Keasling JD (2009) Optimization of the mevalonate-based isoprenoid biosynthetic pathway in Escherichia coli for production of the anti-malarial drug precursor amorpha- 4,11-diene. Metab Eng 11:13-19.

Appel KF, Wolff AM, Arnau J (2004) A multicopy vector system for genetic studies in Mucor circinelloides and other zygomycetes. Mol Genet Genom 271:595-602.

Araújo JP, Hughes DP (2016) Diversity of entomopathogenic fungi: which groups conquered the insect body?. In Advances in genetics (Vol. 94, pp. 1-39), Academic Press.

Arcidiacono S, Kaplan DL (1992) Molecular weight distribution of chitosan isolated from Mucor rouxii under different culture and processing conditions. Biotechnol Bioeng 39:281-286.

Azami Y, Hattori A, Nishimura H, Kawaide H, Yoshimura T, Hemmi H (2014) (R)-mevalonate 3-phosphate is an intermediate of the mevalonate pathway in Thermoplasma acidophilum. J Biol Chem 289:15957-15967.

Azanza JR, Sádada B, Reis J (2015) Liposomal formulations of amphotericin B: differences according to the scientific evidence. Rev Esp Quimioter 206:275-281.

Balance DJ, Buxton FP, Turner G (1983) Transformation of Aspergillus nidulans by the orotidine-5 phosphate decarboxylase gene of Neurospora crassa. Biochem Biophys Res Commun 112:284-289.

Barbosa MS, Cunha Passos DA, Felipe MS, Jesuíno RS, Pereira M, de Almeida Soares CM (2004) The glyceraldehyde-3-phosphate dehydrogenase homologue is differentially regulated in phases of Paracoccidioides brasiliensis: molecular and phylogenetic analysis. Fungal Genet Biol 41:667-675.

Batista NT, Reberšek M, Vernier PT, Mali B, Miklavčič D (2016) Effects of high voltage nanosecond electric pulses on eukaryotic cells (in vitro): A systematic review. Bioelectrochem 110:1-12.

Beggs JD (1978) Transformation of yeast by a replicating hybrid plasmid. Nature 275:104-109.

Begley M, Bron PA, Heuston S, Casey PG, Englert N, Wiesner J, Jomaa H, Gahan CG, Hill C (2008) Analysis of the isoprenoid biosynthesis pathways in Listeria 
monocytogenes reveals a role for the alternative 2-C-methyl-D-erythritol 4-phosphate pathway in murine infection. Infect Immun 76:5392-5401.

Begley M, Gahan CG, Kollas AK, Hintz M, Hill C, Jomaa H, Eberl M (2004) The interplay between classical and alternative isoprenoid biosynthesis controls $\gamma \delta \mathrm{T}$ cell bioactivity of Listeria monocytogenes. FEBS Lett 561:99-104.

Bemis LT, Denis CL (1998) Identification of Functional Regions in the Yeast Transcriptional Activator ADR1. Mol Cell Biol 8:2125-2131.

Benito EP, Díaz-Mínguez JM, Iturriaga EA, Campuzano V, Eslava AP (1992) Cloning and sequence analysis of the Mucor circinelloides pyrG gene encoding orotidine-5'-monophosphate decarboxylase and its use for homologous transformation. Gene 116:59-67.

Benny GL, Humber RA, Morton JB (2001) Zygomycota: Zygomycetes. In: McLaughlin DJ, McLaughlin EG, Lemke PA, eds. The Mycota VII, Systematics and evolution, Part A. Berlin: Springer-Verlag:113-146.

Benny GL, O'Donnell K (2000) Amoebidium parasiticum is a protozoan, not a Trichomycete. Mycologia 92:1133-1137.

Bergman P, Linde C, Putsep K, Pohanka A, Normark S, Henriques-Normark B, Andersson J, Bjorkhem-Bergman L (2011) Studies on the antibacterial effects of statins-in vitro and in vivo. PLoS ONE 6:e24394.

Bhosale P, Bernstein (2005) Microbial xanthophylls. Appl Micobiol Biotechnol 68:445-455.

Bidle KA, Hanson TE, Howell K, Nannen J (2007) HMG-CoA reductase is regulated by salinity at the level of transcription in Haloferax volcanii. Extremophiles 11:49-55.

Bien CM, Espenshade PJ (2010) Sterol regulatory element binding proteins in fungi: hypoxic transcription factors linked to pathogenesis. Eukaryotic Cell 9:352-359.

Bondaryk M, Kurzatkowoski W, Staniszewska M (2013) Antifungal agent commonly used the superficial and mucosal candidiasis treatment: mode of action and resistance development. Postepy Dermatol Alergol 30:293-301.

Britton G, Liaaen-Jensen S, Pfander H (2009) Carotenoids volume 5: nutrition and health. Basel: Birkhäuser; Editors: Britton, George, Liaaen-Jensen, Synnove, Pfander, Hanspeter: DOI 10.1007/978-3-7643-7501-0.

Buhaescu I, Izzedine $\mathbf{H}$ (2007) Mevalonate pathway: A review of clinical and therapeutical implications. Clin Biochem 40:575-584. 
Burg JS, Espenshade PJ (2011) Regulation of HMG-CoA reductase in mammals and yeast. Prog Lipid Res 50:403-410.

C`ertík M, Masrnová S, Sitkey V, Minárik M, Breierová E (2005) Biotechnological production of astaxanthin. Chem Listy 99:237-240.

Cafaro MJ (2005) Eccrinales (Trichomycetes) are not fungi, but a clade of protists at the early divergence of animals and fungi. Mol Phylogenet Evol 35:21-34.

Campoy S, Perez F, Martin JF, Gutierrez S, Liras P (2003) Stable transformants of the azaphilone pigment-producing Monascus purpureus obtained by protoplast transformation and Agrobacterium-mediated DNA transfer. Curr Genet 43:447-452.

Caramalho R, Maurer E, Binder U, Arau'jo R, Dolatabadi S, Lass-Flörl C, Lackner M (2015) Etest cannot be recommended for in vitro susceptibility testing of Mucorales. Antimicrob Agents Chemother 59:3663-3665.

Caramalho R, Tyndall JD, Monk BC, Larentis T, Lass-Flörl C, Lackner M (2017) Intrinsic short-tailed azole resistance in mucormycetes is due to an evolutionary

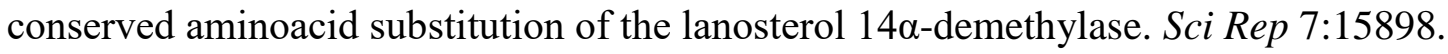

Carrier RL, Milletr LA, Ahmed I (2007) The utility of cyclodextrins for enhancing oral bioavailability. J Control Release 123:78-99.

Carris LM, Little CR, Stiles CM (2012) Introduction to Fungi. The Plant Health Instructor DOI:10.1094/PHI-I-2012-0426-01.

Case ME, Schweizer M, Kushner SR, Giles NH (1979) Efficient transformation of Neurospora crassa by utilizing hybrid plasmid DNA. Proc Natl Acad Sci USA 76:5259-5263.

Centre for Disease Control (CDC) USA (last updated at December 2015) Mucormycosis risk and prevention.

Chang W, Song H, Liu H, Liu P (2013) Current development in isoprenoid precursor biosynthesis and regulation. Curr Opin Chem Biol 17:571-579.

Chapman JR, Waldenström J (2015) With reference to reference genes: A systematic review of endogenous controls in gene expression studies. PLoS One 10:e0141853.

Chávez-Fumagalli MA, Ribeiro TG, Castilho RO Fernandes SO, Cardoso VN, Coelho CS, Mendonça DV, Soto M, Tavares CA, Faraco AA, Coelho EA, Chen M, Poulter CD (2010) Characterization of thermophilic archaeal isopentenyl phosphate kinases. Biochem 49:207-217.

Chayakulkeeree M, Ghannoum MA, Perfect JR (2006) Zygomycosis: the reemerging fungal infection. Eur J Clin Microbiol Infect Dis 25:215-229. 
Cheng P, Yang Y, Wang $\mathbf{L}$, He Q, Liu Y (2003) WHITE COLLAR-1, a multifunctional neurospora protein involved in the circadian feedback loops, light sensing, and transcription repression of wc-2. J Biol Chem 278:3801-3808.

Collins RJ, Harmon BV, Gobe GC, Kerr JFR (1992) Internucleosomal DNA cleavage should not be the sole criterion for identifying apoptosis. Int J Radiat Biol 61:451-453.

Cornely OA, Arikan-Akdagli S, Dannaoui E, Groll AH, Lagrou K et al. (2014) ESCMID and ECMM joint clinical guidelines for the diagnosis and management of mucormycosis. Clin Microbiol Infect 20:5-26.

Corrochano LM, Kuo A, Marcet-Houben M, Polaino S, Salamov A, VillalobosEscobedo JM et al (2016) Expansion of signal transduction pathways in fungi by extensive genome duplication. Curr Biol 26:1577-1584.

Covert SF, Kapoor P, Lee M, Briley A, Nairn CJ (2001) Agrobacterium tumefaciens mediated transformation of Fusarium circinatum. Mycol Res 105:259-264.

Csernetics Á, Nagy G, Iturriaga EA, Szekeres A, Eslava AP, Vágvölgyi CS, Papp T (2011) Expression of three isoprenoid biosynthesis genes and their effects on the carotenoid production of the zygomycetes Mucor circinelloides. Fungal Genet Biol 48:696-703.

Csernetics A, Tóth E, Farkas A, Nagy G, Bencsik O, Vágvölgyi C, Papp T (2015)

Expression of Xanthophyllomyces dendrorhous cytochrome P450 hydoxylase and reductase in Mucor circinelloides. World J Micobiol Biotecnol 31:321-336.

Dandan Li, Tang Y, Lin J, Cai W (2017) Methods for genetic transformation of filamentous fungi. Microb Cell Fact 16:168.

Danial NN, Korsmeyer SJ, (2004) Cell death: critical control points. Cell 116:205219.

Das A, Yoon SH, Lee SH, Kim JY, Oh DK, Kim SW (2007) An update on microbial carotenoid production: application of recent metabolic engineering tools. Appl Microbiol Biotechnol 77:505-512.

de Haro JP, Calo S, Cervantes M, Nicolás FE, Torres-Martínez S, Ruiz-Vázquez RM (2009) A single dicer gene is required for efficient gene silencing associated with two classes of small antisense RNAs in Mucor circinelloides. Eukaryot Cell 10:14861497. 
DeGraw AJ, Hast MA, Xu J, Mullen D, Beese LS, Barany G, Distefano MD (2008) Caged protein prenyltransferase substrates, tools for understanding protein prenylation. Chem Biol Drug Des 72:171-181.

Dellas N, Thomas ST, Manning G, Noel JP (2013) Discovery of a metabolic alternative to the classical mevalonate pathway. Elife 2:e00672.

Dixon DM, Walsh TJ (1996) Antifungal agents. In: Medical Microbiology. $4^{\text {th }}$ edition (ed. Baron S) Galveston (TX): University of Texas Medical Branch at Galveston.

Drogari-Apiranthitou M, Mantopoulou FD, Skiada A, Kanioura L, Grammatikou M, Vrioni G, Mitroussia-Ziouva A, Tsakris A, Petrikkos G (2012) In vitro antifungal susceptibility of filamentous fungi causing rare infections: synergy testing of amphotericin B, posaconazole and anidulafungin in pairs. $J$ Antimicrob Chemother 67:1937-1940.

Ducrey Sanpietro LM, Kula MR (1998) Studies of astaxanthin biosynthesis in Xanthophyllomyces dendrorhous (Phaffia rhodozyma). Effect of inhibitors and low temperature. Yeast 14:1007-1016.

Dupont S, Lemetais G, Ferreira T, Cayot P, Gervais P, Beney L (2012) Ergosterol biosynthesis: a fungal pathway for life on land. Evolution 66:2961-2968.

Eagle H, Musselman AD (1948) The rate of bactericidal action of penicillin in vitro as a function of its concentration, and its paradoxically reduced activity at high concentrations against certain organisms. J Exp Med 88:99.

Eamens A, Wang MB, Smith NA, Waterhouse PM (2008) RNA silencing in plants: yesterday, today, and tomorrow. Plant physiology 147:456-468.

Eslava AP, Alvarez MI, Delbrck M (1975) Meiosis in Phycomyces. Pro Natl Acad Sci USA 72:4076-4080.

Ferreira ME, Colombo AL, Paulsen I, Ren Q, Wortman J, Huang J, Goldman MH, Goldman GH (2005) The ergosterol biosynthesis pathway, transporter genes, and azole resistance in Aspergillus fumigatus. Med Mycol 43:S313-S319.

Fincham JRS (1989) Transformation in fungi. Microbio Rev 53:148-170.

Franz R, Kelly SL, Lamb DC, Kelly DE, Ruhnke M, Morschhäuser J (1998) Multiple molecular mechanisms contribute to a stepwise development of fluconazole resistance in clinical Candida albicans strains. Antimicrob Agents Chemother 42:30653072 . 
Fuller KK, Chen S, Loros JJ, Dunlap JC (2015) Development of the CRISPR/Cas9 system for targeted gene disruption in Aspergillus fumigatus. Eukaryot Cell 14:10731080.

Galea AM, Brown AJ (2009) The special relationship between sterols and oxygen: were sterols an adaptation to aerobic life?. Free Radic Biol Med 47:880-889.

Galgóczy L, Nyilasi I, Papp T, Vágvölgyi C (2011) Statins as antifungal agents. World J Clin Infect Dis 1:4-10.

Garre V, Nicolas FE, Torres-Martínez S, Ruiz-Vazquez RM (2014) The RNAi machinery in Mucorales: the emerging role of endogenous small RNAs. In A. Sesma, and von der Haar T. (Eds.), Fungal RNA biology (pp. 291e313). Cham: Springer International Publishing Switzerland.

Georgopapadakou NH, Bertasso A (1992) Effects of squalene epoxidase inhibitors on Candida albicans. Antimicrob Agent Therap 36:1779-1781.

Gladieux P, Ropars J, Badouin H, Branca A, Aguileta G, De Vienne DM, Rodriguez de la Vega RC, Branco S, Giraud T (2014) Fungal evolutionary genomics provides insight into the mechanisms of adaptive divergence in eukaryotes. Mol Ecol 23:753-773.

Gomes MZ, Lewis RE, Kontoyiannis DP (2011) Mucormycosis caused by unusual mucormycetes, non-rhizopus, Mucor, and Lichtheimia species. Clin Microbiol Rev 24:411-445.

Gonzalez-Hernandez GA, Herrera-Estrella L, Rocha-Ramirez V, Roncero MIG, Gutierrez-Corona JF (1997) Biolistic transformation of Mucor circinelloides. Mycological research 101:953-956.

Gooday GW, Adams DJ (1993) Sex hormones and fungi. Advances in Microbial Physiology 34:69-145.

Gordon PA, Stewart PR, Clark-Walker DG (1970) Fatty acid and sterol composition of Mucor genevensis in relation to dimorphism and anaerobic growth. J Bacteriol 107:114-120.

Gougouli M, Kalantzi K, Beletsiotis E, Koutsoumanis KP (2011) Development and application of predictive models for fungal growth as tools to improve quality control in yogurt production. Food Microbiol 28:1453-1462.

Green DR (2005) Apoptotic pathways: ten minutes to dead. Cell 121:671-674. 
Greenberg RN, Mullane K, van Burik JA, Raad I, Abzug MJ et al (2006) Posaconazole as salvage therapy for zygomycosis. Antimicrob Agents Chemother 50:126-133.

Grochowski LL, Xu H, White RH (2006) Methanocaldococcus jannaschii uses a modified mevalonate pathway for biosynthesis of isopentenyl diphosphate. J Bacteriol 188:3192-3198.

Gutierrez A, Lopez-Garcia S, Garre V (2011) High reliability transformation of the basal fungus Mucor circinelloides by electroporation. J Micribiol Method 84:442-446.

Gyetvai Á, Emri T, Takács K, Dergez T, Fekete A, Pesti M, Lenkey B (2006) Lovastatin possesses a fungistatic effect against Candida albicans, but does not trigger apoptosis in this opportunistic human pathogen. FEMS Yeast Res 6:1140-1148.

Harms H, Schlosser D, Wick LY (2011) Untapped potential: exploiting fungi in bioremediation of harzadous chemical. Nat Rev Microbiol 9177-9192.

Harrison CJ, Bohm AA, Nelson HCM (1994) Crystal structure of the DNA binding domain of the heat shock transcription factor. Science 263:224-227.

He Q, Liu Y (2005) Molecular mechanism of light responses in Neurospora: from light-induced transcription to photoadaptation. Genes Dev 19:2888-2899.

Hemmerlin A, Harwood JL, Bach TJ (2012) A raison d'être for two distinct pathways in the early steps of plant isoprenoid biosynthesis? Prog Lipid Res 51:95-148.

Heuston S, Begley M, Gahan CGM, Hill C (2012) Isoprenoid biosynthesis in bacterial pathogens. Microbiology 158:1389-1401.

Hibbett DS, Binder M, Bischoff JF, Blackwell M, Cannon PF et al (2007) A higherlevel phylogenetic classification of the fungi. Mycol Res 111:509-547.

Hinnen A, Hicks JB, Fink GR (1978) Transformation of yeast. Proc Natl Acad Sci USA 75:1929-1933.

Hirano T, Sato T, Yaegashi K, Enei H (2000) Efficient transformation of the edible basidiomycete Lentinus edodes with a vector using a glyceraldehyde-3-phosphate dehydrogenase promoter to hygromycin B resistance. Mol Gen Genet 263:1047-1052.

Hoffmann K, Voigt K, Kirk PM (2011) Mortierellomycotina subphyl. nov., based on multigene genealogies. Mycotaxon 115:353-363.

Hood E (2004) RNAi: What's all the noise about gene silencing?. Environ Health Perspect 112:A224-A229.

Howard SJ, Arendrup MC (2011) Acquired antifungal drug resistance in Aspergillus fumigatus: epidemiology and detection. Med Mycol 49:S90-S95. 
Hussein G, Sankawa U, Goto H, Matsumoto K, Watanabe H (2006) Astaxanthin, a carotenoid with potential in human health and nutrition. J Nat Prod 69:443-449.

Ibrahim AS, Skory CD (2006) Genetic manipulation of Zygomycetes in Medical mycology: cellular and molecular techniques. (ed. Kavanagh, K.):305-326.

Ibrahim AS, Spellberg B Edwards J (2008) Iron acquisition: A novel prospective on mucormycosis pathogenesis and treatment. Curr Opin Infect Dis 21:620-625.

Ibrahim AS, Spellberg B, Walsh TJ, Kontoyiannis DP (2012) Pathogenesis of mucormycosis. Cin Infect Dis 54:16-22.

Idnurm A (2011) Sex determination in the first-described sexual fungus. Eukaryot cell 10:1485-1491.

Iigusa H, Yoshida Y, Hasunuma K (2005) Oxygen and hydrogen peroxide enhance light-induced carotenoid synthesis in Neurospora crassa. FEBS Lett 579:4012-4016.

Istvan ES, Deisenhofer J (2001) Structural mechanism for statin inhibition of HMGCoA reductase. Science 292:1160-64.

Iturriaga EA, Velayos A, Eslava AP (2000) The structure and function of the genes involved in the biosynthesis of carotenoids in the Mucorales. Biotechnol Bioprocess Eng 5:263-274.

James TY, Kerry O (2004) Zygomycota. Microscopic 'Pin' or 'Sugar' Molds. Version 21 December 2004 (complete) http://tolweb.org/Zygomycota/20518/2004.12. 12.21 in The Tree of Life Web Project, http://tolweb.org/.

Joseph HT, Hollomon D, Loeffler RS, Kelly SL (1995) Cross-resistance to polyene and azole drugs in Cryptococcus neoformans. Antimicrob Agents Chemother 39:15261539 .

Joseph-Horne T, Hollomon DW (1997) Molecular mechanism of azole resistance in fungi. FEMS Microbiol Lett 149:141-149.

Jung MK, Ovechkina Y, Prigozhina N, Oakley CE, Oakley BR (2000) The use of D-glucanase as a substitute for Novozym 234 in immunofluorescence and protoplasting. Fungal Genet Newslett 47:65-66.

Kadotani N, Nakayashiki H, Tosa Y, Mayama S (2003) RNA silencing in the phytopathogenic fungus Magnaporthe oryzae. Mol Plant Microb Interact 16:769-776.

Karimi K, and Zamini A (2013) Mucor indicus: biology and industrial application perspective: a review Biotechnol Adv 31:466-481.

Kelly SL, Lamb DC, Taylor M, Corran AJ, Baldwin BC, Powderly WG (1994) Resistance to amphotericin B associated with defective sterol D 8r7 isomerase in a 
Cryptococcus neoformans strain from an AIDS patient. FEMS Microbiol Lett 122:39_ 42.

Kraibooj K, Park HR, Dahse HM, Skerka C, Voigt K, Figge MT (2014) Virulent strain of Lichtheimia corymbifera shows increased phagocytosis by macrophages as revealed by automated microscopy image analysis. Mycoses 57:56-66.

Kuwano T, Shirataki C, Itoh Y (2008) Comparison between polyethylene glycol- and polyethylenimine-mediated transformation of Aspergillus nidulans. Curr Genet 54:95103.

Kuzina V, Cerda-Olmedo E (2007) Ubiquinone and carotene production in the Mucorales Blakeslea and Phycomyces. Appl Microbiol Biotechnol 76:991-999.

Kwon-Chung KJ (2012) Taxonomy of fungi causing mucormycosis and entomophthoramycosis (zygomycosis) and nomenclature of the disease: molecular mycologic perspectives. Clin Infect Dis 54:8-15.

Lamers PP, Janssen M, De Vos RC, Bino RJ, Wijels RH (2008) Exploring and exploiting carotenoid accumulation in Dunaliella salina for cell factory applications. Trends Biotechnol 26:631-638.

Larsen GG, Appel KF, Wolff A (2004) Characterisation of the Mucor circinelloides regulated promoter gpd1P. Curr Genet 45:225-234.

Lee PC, Schmidt-Dannert C (2002) Metabolic engineering towards biotechnological production of carotenoids in microorganisms. Appl Microbiol Biotechnol 60:1-11.

Lee SC, Li A, Calo S, Inoue M, Tonthat NK, Bain JM, Louw J, Shinohara ML, Erwig LP, Schumacher MA, Ko DC, Heitman J (2015) Calcineurin orchestrates dimorphic transitions, antifungal drug responses and host-pathogen interactions of the pathogenic mucoralean fungus Mucor circinelloides. Mol Microbiol 97:844-865.

Lemke A, Kiderlen AF, Kayser O (2005) Amphotericin B. Appl Microbiol Biotechnol 68:151-62.

Lepak AJ, Andes DR (2015) Antifungal Pharmacokinetics and Pharmacodynamics. Cold Spring Harb Perspect Med 5:a019653.

Li CH, Cervantes M, Springer DJ, Boekhout T, Ruiz-Vazquez RM, TorresMartinez, SR, Lee SC (2011) Sporangiospore size dimorphism is linked to virulence of Mucor circinelloides. PLoS pathogens 7:e1002086.

Li D, Tang Y, Lin J, Cai W (2017) Methods for genetic transformation of filamentous fungi. Microb Cell Fact 16:168. 
Li L, Chang SS, Liu Y (2010) RNA interference pathways in filamentous fungi. Cell Mol Life Sci 67:3849-3863.

Li Y, Wadsö L, Larsson L (2009) Impact of temperature on growth and metabolic efficiency of Penicillium roqueforti; correlations between produced heat, ergosterol content and biomass. J App Microbiol 106:1494-1502.

Liang PH, Ko TP, Wang AHJ (2002) Structure, mechanism and function of prenyltransferases. Eur J Biochem 269:3339-3354.

Liao P, Hemmerlin A, Bach TJ, Chye ML (2016) The potential of the mevalonate pathway for enhanced isoprenoid production. Biotechnol Adv 34:697-713.

Lima JO, dos Santos JK, Pereira JF, de Resende ML, de Araújo EF, de Queiroz MV (2003) Development of a transformation system for Crinipellis perniciosa, the causal agent of witches' broom in cocoa plants. Curr Genet 42:236-240.

Lin X, Momany M (2004) Identification and complementation of abnormal hyphal branch mutants ahbAl and ahbB1 in Aspergillus nidulans. Fungal Genet Biol 41:9981006.

Linden H, Ballario P, Macino G (1997) Blue light regulation in Neurospora crassa. Fungal Genet Biol 22:141-150.

Liu H, Cottrell TR, Pierini LM, Goldman WE, Doering TL (2002) RNA interference in the pathogenic fungus Cryptococcus neoformans. Genetics 160:463-470. Livak KJ, Schmittgen TD (2001) Analysis of relative gene expression data using real time quantitative PCR and the 2(-Delta Delta C(T)) method. Methods 25:402-408.

Lo Re V, III, Carbonari DM, Lewis JD, Forde KA, Goldberg DS, Reddy KR, Haynes K, Roy JA, Sha D, Marks AR, Schneider JL, Strom BL, Corley DA (2016) Oral azole antifungal medications and risk of acute liver injury, overall and by chronic liver disease status. Am J Med 129:283-291.

Lombraña M, Moralejo FJ, Pinto R, Martín JF (2004) Modulation of Aspergillus awamori thaumatin secretion by modification of bipA gene expression. Appl Environ Microbiol 70:5145-5152.

Long N, Xu X, Zeng Q, Sang H, Lu L (2017) Erg4A and Erg4B are required for conidiation and azole resistance via regulation of ergosterol biosynthesis in Aspergillus fumigatus. Appl Environ Microbiol 83:e2924-16.

Lopes FJF, Queiroz MV, Lima JO, Silva VAO, Araújo EF (2008) Restriction enzyme improves the efficiency of genetic transformations in Crinipellis perniciosa, the 
causal agent of witches' broom disease in Theobroma cacao. Braz Arch Biol Technol $51: 27-34$.

Lorenz RT, Parks LW (1990) Effects of lovastatin (mevinolin) on sterol levels and on activity of azoles in Saccharomyces cerevisiae. Antimicrob Agents Chemother 34:16601665.

Loyse A, Dromer F, Day J, Lortholary O, Harrison TS (2013) Flucytosine and cryptococcosis: time to urgently address the worldwide accessibility of a 50-year-old antifungal. J Antimicrob Chemother 68:2435-2444.

Lukács Gy, Papp T, Nyilasi I, Nagy E, Vágvölgyi C (2004) Differentiation of Rhizomucor species on the basis of their different sensitivities to lovastatin. $J$ clin microbiol 42:5400-5402.

MacCallum DM, Coste A, Ischer F, Jacobsen MD, Odds FC, Sanglard D (2010) "Genetic dissection of azole resistance mechanisms in Candida albicans and their validation in a mouse model of disseminated infection,"Antimicrob Agents Chemother 54:1476-1483.

Maciejak A, Leszczynska A, Warchol I, Gora M, Kaminska J et al (2013) The effects of statins on mevalonic acid pathway in recombinant yeast strains expressing human HMG-CoA reductase. BMC Biotechnol 30:13:68.

Majumdar R, Rajasekaran K, Cary JW (2017) RNA Interference (RNAi) as a potential tool for control of mycotoxin contamination in crop plants: concepts and considerations. Front Plant Sci 14:200.

Malonek S, Meinhardt F (2001) Agrobacterium tumefaciens-mediated genetic transformation of the phytopathogenic ascomycete Calonectria morganii. Curr Genet 40:152-155.

Makushok T, Alves P, Huisman SM, Kijowski AR, Brunner D (2016) Sterol-rich membrane domains define fission yeast cell polarity. Cell 165:1182-1196.

Mans R, van Rossum HM, Wijsman M, Backx A, Kuijpers NG, van den Broek M, Daran-Lapujade P, Pronk JT, van Maris AJ, Daran JM (2015) CRISPR/Cas9: a molecular Swiss army knife for simultaneous introduction of a multiple genetic modifications in Saccharomyces cerevisiae. FEMS Yeast Res 15:fov004.

Mantzouridou F, Roukas T, Kotzekidou P (2002) Effect of the aeration rate and agitation speed on beta-carotene production and morphology of Blakeslea trispora in a stirred tank reactor: mathematical modeling. Biochem Eng J 10:123-135. 
Marcoleta A, Niklitschek M, Wozniak A, Lozano C, Alcaíno J, Baeza M, Cifuentes V (2011) Glucose and ethanol-dependent transcriptional regulation of the astaxanthin biosynthesis pathway in Xanthophyllomyces dendrorhous. BMC Microbiol 11:190.

Marr KA, Lyons CN, Rustad T, Bowden RA, White TC (1998) "Rapid, transient fluconazole resistance in Candida albicans is associated with increased mRNA levels of CDR," Antimicrob Agents Chemother 42:2584-2589.

Marty FM, Ostrosky-Zeichner L, Cornely OA, Mullane KM, Perfect JR et al (2016) Isavuconazole treatment for mucormycosis: a single-arm open-label trial and case-control analysis. Lancet Infect Dis 16:828-837.

Masadeh M, Mhaidat N, Alzoubik K, Alazzams S, Alnasser Z (2012) Antibacterial activity of statins: a comparative study of atorvastatin, simvastatin, and rosuvastatin. Ann Clin Micro biol Antimicrob 2012:11-13.

Mehta BJ, Obraztsova IN, Cerdá-Olmedo E (2003) Mutants and intersexual heterokaryons of Blakeslea trispora for production of $\beta$-carotene and lycopene. Appl Envir Microbiol 69:4043-4048.

Mélida H, Sain D, Stajich JE, Bulone V (2015) Deciphering the uniqueness of Mucoromycotina cell walls by combining biochemical and phylogenomic approaches. Environ Microbiol 17:1649-1662.

Mertens JA, Skory CD, Ibrahim AS (2006) Plasmids for expression of heterologous proteins in Rhizopus oryzae. Arch Microbiol 186:41-50.

Mesa-Arango AC, Rueda C, Román E, Quintin J, Terrón MC, Luque D, Netea M, Pla J, Zaragoza O (2016) Cell wall changes in amphotericin B-resistant stain from Candida tropcalis and relationship with immune response elicted by the host. Antimicrob Agents Chemother 60:2326-2335.

Meyer V (2008) Genetic engineering of filamentous fungi Progress, obstacles and future trends. Biotechnol Advances 26:177-185.

Miao L, Chi S, Tang Y, Su Z, Yin T, Guan G, Li Y (2011) Astaxanthin biosynthesis is enhanced by high carotenogenic gene expression and decrease of fatty acids and ergosterol in a Phaffia rhodozyma mutant strain. FEMS Yeast Res 11:192-201.

Michielse CB, Salim K, Ragas P, Ram AF, Kudla B, Jarry B, Punt PJ, van den Hondel CA (2004) Development of a system for integrative and stable transformation of the zygomycete Rhizopus oryzae by Agrobacterium-mediated DNA transfer. Mol Genet Genomics 271:499-510. 
Middleton B (1972) The kinetic mechanism of 3-hydroxy-3-methylglutaryl-coenzyme A synthase from baker's yeast. Biochem J 126:35-47.

Mikosch TS, Lavrijssen B, Sonnenberg AS, van Griensven LJ (2001) Transformation of the cultivated mushroom Agaricus bisporus (LANGE) using T-DNA from Agrobacterium tumefaciens. Curr Genet 39:35-39.

Millati R, Edebo L, Taherzadeh MJ (2005) Performance of Rhizopus, Rhizomucor, and Mucor in ethanol production from glucose, xylose, and wood hydrolyzates. Enzyme Microb Technol 36:294-300.

Misawa N, Shimada H (1997) Metabolic engineering for the production of carotenoids in non-carotenogenic bacteria and yeasts. J Biotechnol 59:169-181.

Miziorko HM, (2011) Enzymes of the mevalonate pathway of isoprenoid biosynthesis. Arch Biochem Biophys 505:131-143.

Morace G, Borghi E (2012) Invasive mold infections: virulence and pathogenesis of Mucorales. Int J Microbiol ID:349278 .

Moralejo FJ, Cardoza RE, Gutierrez S, Lombraña M, Fierro F, Martín JF (2002)

Silencing of the aspergillopepsin B (pepB) gene of Aspergillus awamori by antisense RNA expression or protease removal by gene disruption results in a large increase in thaumatin production. Applied Environment Microbiol 68:3550-3559.

Morelli G, Nelson MA, Ballario P, Macino G (1993) Photoregulated carotenoid biosynthetic genes of Neurospora crassa. Methods Enzymol 214:412-424.

Mosqueda-Cano G, Gutierrez-Cororna JF (1995) Environmental and developmental regulation of carotenogenesis in the dimorphic fungus Mucor rouxii. Curr Microbiol 31:141-145.

Mouyna I, Henry C, Doering TL, Latgé JP (2004) Gene silencing with RNA interference in the human pathogenic fungus Aspergillus fumigatus. FEMS Microbiol Lett 237:317-324.

Mullins ED, Chen X, Romaine P, Raina R, Geiser DM, Kang S (2001) Agrobacterium-mediated transformation of Fusarium oxysporum: an efficient tool for insertional mutagenesis and gene transfer. Phytopathol 91:173-180.

Muszewska A, Pilsyk S, Perlińska-Lenart U, Kruszewska JS (2017) Diversity of cell wall related proteins in human pathogenic fungi. J Fungi 4:6.

Nagy A, Vágvölgyi C, Balla E, Ferenczy L (1994) Electrophoretic karyotype of Mucor circinelloides. Curr Genet 26:45. 
Nagy G, Csernetics Á, Bencsik O, Szekeres A, Vágvölgyi CS, Papp T (2012) Carotenoid composition of Mucorales fungi. Afr J Microbiol Res 6:7265-7270.

Nagy G, Farkas A, Csernetics Á, Bencsik O, Szekeres A, Nyilasi I, Vágvölgyi C, Papp T (2014) Transcription of the three HMG-CoA reductase genes of Mucor circinelloides. BMC Microbiol 14:93.

Nagy G, Szebenyi C, Csernetics Á, Vaz AG, Tóth EJ, Vágvölgyi C, Papp T (2017) Development of a plasmid free CRISPR-Cas9 system for the genetic modification of Mucor circinelloides. Sci Rep 7:16800.

Nakayashiki H (2005) RNA silencing in fungi: mechanisms and applications. FEBS Lett 579:5950-5957.

Navarro E, Lorca-Pascual JM, Quiles-Rosillo MD, Nicolás FE, Garre V, TorresMartínez S, Ruiz-Vázquez RM (2001) A negative regulator of light inducible carotenogenesis in Mucor circinelloides. Mol Genet Genomics 266:463-470.

Nicolás FE, Torres-Martínez S, Ruiz-Vázquez RM, (2003) Two classes of small antisense RNAs in fungal RNA silencing triggered by non-integrative transgenes. EMBO J 22:3983-3991.

Nicolas FE, Vila A, Moxon S, Cascales MD, Torres-Martínez S, Ruiz-Vázquez RM, Garre V (2015) The RNAi machinery controls distinct responses to environmental signals in the basal fungus Mucor circinelloides. BMC Genomics 16:237. Nielsen JC, Nielsen J (2017) Development of fungal cell factories for the production of secondary metabolites: linking genomics and metabolism. Synth Syst Biotechnol 2:512.

Nødvig CS, Nielsen JB, Kogle ME, Mortensen UH (2015) A CRISPR-Cas9 system for genetic engineering of filamentous fungi. PLoS One 10:e0133085.

Nyilasi I, Acs K, Papp T, Nagy E, Vagvolgyi C (2005) Agrobacterium tumefaciensmediated transformation of Mucor circinelloides. Folia Microbiologica 50:415-420.

Nyilasi I, Kocsubé S, Krizsán K, Galgóczy L, Papp T, Pesti M, Nagy K, Vágvölgyi C (2014) Susceptibility of clinically important dermatophytes against statins and different statin-antifungal combinations. Med Mycol 52:140-148.

Oh J, Kim JH, Park JG, Yi YS, Park KW, Rho HS, Lee MS, Yoo JW, Kang SH, Hong YD, Shin SS, Cho JY (2013) Radical scavenging activity-based and AP-1targeted anti-inflammatory effects of lutein in macrophage-like and skin keratinocytic cells. Mediat Inflamm 787042. 
Oide S, Moeder W, Krasnoff S, Gibson D, Haas H, Yoshioka K, Turgeon BG (2006) NPS6, encoding a nonribosomal peptide synthetase involved in siderophoremediated iron metabolism, is a conserved virulence determinant of plant pathogenic ascomycetes. Plant Cell 18:2836-2853.

Orset S, Young AJ (1999) Low-temperature-induced synthesis of $\alpha$-carotene in the microalga Dunaliella salina (chlorophyta). J Phycology 35:520-527.

Pagano L, Cornely OA, Busca A, Caira M, Cesaro S, Gasbarrino C et al. (2013) Combined antifungal approach for the treatment of invasive mucormycosis in patients with hematologic diseases: a report from the SEIFEM and FUNGISCOPE registries. Haematologica 98:e127-30.

Palmer CNA, Hsu MH, Griffin KJ, Johnson EF (1995) Novel sequence determinants in peroxisome proliferator signaling. J Biol Chem 270:16114-16121.

Papaioannou EH, Liakopoulou-Kyriakides (2012) Agro food waste utilization by Blackeslea trispora for carotenoids production. Acta Biochim Pol 59:151-153.

Papp T, Csernetics A, Nagy G, Bencsik O, Iturriaga EA, Eslava AP, Vágvölgyi C (2013) Canthaxanthin production with modified Mucor circinelloides strains. Appl Microbiol Biotechnol 97:4937-4950.

Papp T, Csernetics Á, Nyilasi I, Ábrók M, Vágvölgyi C (2010) Genetic transformation of Zygomycetes fungi. In Progress in Mycology (pp. 75-94), Springer, Dordrecht.

Papp T, Velayos A, Bartok T, Eslava AP, Vagvolgyi C, Iturriaga EA (2006) Heterologous expression of astaxanthin biosynthesis genes in Mucor circinelloides. Applied Microbiol Biotechnol 69:526-531.

Perea S, López-Ribot JL, Kirkpatrick WR, McAtee RK, Santillán RA, Martínez M, Calabrese D, Sanglard D, Patterson TF (2001) "Prevalence of molecular mechanisms of resistance to azole antifungal agents in Candida albicans strains displaying high-level fluconazole resistance isolated from human immunodeficiency virus-infected patients," Antimicrob Agents Chemother 45:2676-2684.

Pfaller MA, Diekema DJ (2005) Unusual fungal and pseudofungal infection of humans. J Clin Mirobiol 43:1495-504.

Platauf, AP (1885) Mycosis mucorina. Virchows Arch 102:543-564.

Prabhu RM, Patel R (2004) Mucormycosis and entomophthoramycosis: a review of the clinical manifestations, diagnosis and treatment. Clin Micobiol Infect 1:31-47. 
Qi XF, Zheng L, Lee KJ, Kim DH, Kim CS, Cai DQ, Wu Z, Qin JW, Yu YH, Kim

SK (2013) HMG-CoA reductase inhibitors induce apoptosis of lymphoma cells by promoting ROS generation and regulating Akt, Erk and p38 signals via suppression of mevalonate pathway. Cell Death Dis 28:4:e518.

Redberry GW (2006) Gene silencing: new research, Grace W. Redberry (editor). New York: Nova Science Publishers.

Rep M, Krantz M, Thevelein JM, Hohmann S (2000) The transcriptional response of Saccharomyces cerevisiae to osmotic shock. Hot1p and Msn2p/Msn4p are required for the induction of subsets of high osmolarity glycerol pathway dependent genes. J Biol Chem 275:8290-8300.

Ribes JA, Vanover-Sams CL, Baker DJ (2000) Zygomycetes in human disease Clin Microbiol Rev 13:236-301.

Richardson M (2009) The ecology of the Zygomycetes and its impact on environmental exposure. Clin Microbiol Infect 5:2-9.

Riley TT, Muzny CA, Swiatlo E, Legendre DP (2016) Breaking the mold: A review of mucormycosis and current pharmacological treatment options. Ann Pharmacother 50:747-757.

Ringel AE, Ryznar R, Picariello H, Huang K, Lazarus AG, Holmes SG (2013) Yeast Tdh3 (Glyceraldehyde 3-Phosphate Dehydrogenase) Is a Sir2 Interacting factor that regulates transcriptional silencing and rDNA recombination. PLoS Genet 9:e1003871.

Rodriguez-Amaya DB, Kimura M (2004) Harvestplus Handbook for Carotenoid Analysis, HarvestPlus Technical Monograph Series 2; IFPRI: Washington, DC, USA, and CIAT: Cali, Colombia.

Roncero MIG, Jepsen LP, Strøman P, van Heeswijck R (1989) Characterization of a leuA gene and an ARS element from Mucor circinelloides. Gene 84:335-343.

Rosenfeld E, Beauvoit B (2003) Role of the non-respiratory pathways in the utilization of molecular oxygen by Saccharomyces cerevisiae. Yeast 20:1115-1144.

Roze LV, Linz JE (1998) Lovastatin triggers an apoptosis-like cell death process in the fungus Mucor racemosus. Fungal Genet Biol 25:119-133.

Ruiz-Diez B, (2002) Strategies for the transformation of filamentous fungi. J Appl Microbiol 92:189-195. 
Ruiz-Vázquez RM, Nicolás FE, Torres-Martínez S, Garre V (2015) Chapter twodistinct RNAi pathways in the regulation of physiology and development in the fungus Mucor circinelloides. Adv Genet 91:55-102.

Salas V, Pastor FJ, Calvo E, Alvarez E, Sutton DA, Mayayo E, Fothergill AW, Rinaldi MG, Guarro J (2012) In vitro and in vivo activities of posaconazole and amphotericin B in a murine invasive infection by Mucor circinelloides: poor efficacy of posaconazole. Antimicrob Agents Chemother 56:2246-2250.

Sambrook J, Fitsch EF, Maniatis T (1989) Molecular cloning: a laboratory manual. Cold Spring Harbor Press, Cold Spring Harbor.

Sandmann G (1994) Carotenoid biosynthesis in microorganisms and plants. Eur $J$ Biochem 223:7-24.

Sanglard D (2002) "Clinical relevance of mechanisms of antifungal drug resistance in yeasts," Enfermedades Infecciosasy Microbiol Clinica 20:462-479.

Sant DG, Tupe SG, Ramana CV, Deshpande MV (2016) Fungal cell membrane promising drug target for antifungal therapy. J Appl Microbiol 121:1498-1510.

Sautour M, Soares Mansur C, Divies C, Benssousan M, Dantigny P (2002) Comparison of the effects of temperature and water activity on growth rate of food spoilage moulds. J Ind Microbiol Biotechnol 28:311-315.

Schachtschabel D, David A, Menzel K-D, Schimek C, Wöstemeyer J, Boland W (2008) Cooperative biosynthesis of trisporoids by the $(+)$ and $(-)$ mating types of the zygomycete Blakeslea trispora. Chembiochem 9:3004-3012.

Schmidt-Dannert C (2015) Biosynthesis of terpenoid natural products in fungi. $A d v$ Biochem Eng Biotechnol 148:19-61.

Schrettl M, Kim HS, Eisendle M, Kragl C, Nierman WC, Heinekamp T, WernerER, Jacobsen I, Illmer P, Yi H, Brakkhage AA, Haas H (2008) SreAmediated iron regulation in Aspergillus fumigatus. Mol Microbiol 70:27-43.

Schumacher J (2017) How light affects the life of Botrytis. Fung Genet Biol 106:2641.

Schumann U, Ayliffe M, Kazan K, Wang MB (2010) RNA silencing in fungi. Frontiers Biol 5:478-494.

Selvakumar D, Miyamoto M, Furuichi Y, Komiyama T (2006) Inhibition of fungal $\beta$-1,3-glucan synthase and cell growth by HM-1 killer toxin single-chain anti-idiotypic antibodies. Antimicrob Agents Chemother 50:3090-3097. 
Seo K, Akiyoshi H, Ohnishi Y (1999) Alteration of cell wall composition leads to amphotericin B resistance in Aspergillus flavus. Microbiol Immunol 43:1017-1025.

Sheng C, Zhenyuan M, Ji H, Yao J, Wang W, Che X, Dong G, Lu J, Gio W, Zhang W (2009) Three-dimentional model of lanosterol- $\alpha$-demethylase from Cryptococcus neoformans: active-site characterization and insights into azole binding. Antimicrob Agents Chemother 53:3487- 3495.

Shetty PK, Sohn HY, Shin KS, Kim E, Seo BI (2000) Biodegradation of cyclodiene insecticide endosulfan by Mucor thermohyalospora MTCC 1384. Curr Sci 79:13811383.

Shields RK, Nguyen MH, Du C, Press E, Cheng S, Clancy CJ (2011) Paradoxical effect of caspofungin against Candida bloodstream isolates is mediated by multiple pathways but eliminated in human serum. Antimicrob Agents Chemother 55:2641-2647. Shrivastava S, Chattopadhyay A (2007) Influence of cholesterol and ergosterol on membrane dynamics using different fluorescent reporter probes. Biochem Biophys Res Commun 356:705-710.

Silva F, Torres-Martínez S, Garre V (2006) Distinct white collar-1 genes control specific light responses in Mucor circinelloides. Mol Microbiol 61:1023-1037.

Simister RL, Poutasse CM, Thurston AM, Reeve JL, Baker MC, White HK (2015) Degradation of oil by fungi isolated from Gulf of Mexico beaches. Mar Pollut Bull 100:327-333.

Simova ED, Frengova GI, Beshkova DM (2004) Synthesis of carotenoids by Rhodotorula rubra GED8 co-cultured with yogurt starter cultures in whey ultrafiltrate. $J$ Ind Microbiol Biotechnol 31:115-121.

Spatafora JW, Chang Y, Benny GL, Lazarus K, Smith ME, Berbee ML, Bonito G, Corradi N, Grigoriev I, Gryganskyi A, James TY, O'Donnell K, Roberson RW, Taylor TN, Uehling J, Vilgalys R, White MM, Stajich JE (2016) A phylum-level phylogenetic classification of zygomycete fungi based on genome-scale data. Mycologia 108:1028-1046.

Stancu C, Sima A (2001) Statins: mechanism of action and effects. J Cell Mol Med $5: 78-387$.

Stover KR, Cleary JD (2015) The Eagle-like effect of the echinocandins: is it relevant for clinical decisions? Curr Fungal Infect Rep 9:88-93.

Sudoh M, Yamazaki T, Masubuchi K, Taniguchi M, Shimma N, Arisawa M, Yamada-Okabe H (2000) Identification of a novel inhibitor specific to the fungal 
chitin synthase; inhibition of chitin synthase 1 arrests the cell growth, but that of chitin synthase 1 and 2 is lethal in the pathogenic fungus Candida albicans. J Biol Chem 275:32901-32905.

Sugar AM (2000) Agents of mucormycosis and related species. In: Mandell, GI, BennettJE, DolinR, eds. Mandell, Douglas, and Bennett's Principles and Practice of Infectious Diseases, 5th edn. New York: Churchill Livingstone; 2685-2695.

Sugar AM, Liu XP (2000) Combination antifungal therapy in treatment of murine pulmonary mucormycosis: roles of quinolones and azoles. Antimicrob Agent Chemotherap 44:2004-2016.

Sutter RP (1970) Effect of light on beta-carotene accumulation in Blakeslea trispora. J Gen Microbiol 64:215-221.

Szkopińska A, Płochocka D (2005) Farnesyl diphosphate synthase: regulation of product specificity. Acta Biochim Pol 52:45-55.

Tabata K, Hashimoto SI (2004) Production of mevalonate by a metabolicallyengineered Escherichia coli. Biotechnol Lett 26:1487-1491.

Tanabe Y, Saikawa M, Watanabe MM, Sugiyama J (2004) Molecular phylogeny of Zygomycota based on EF-1 and RPB1 sequences: limitations and utility of alternative markers to rDNA. Mol Phylogenet Evol 30:438-449.

Tanabe Y, Watanabe MM, Sugiyama J (2005) Evolutionary relationships among basal fungi (Chytridiomycota and Zygomycota): Insights from molecular phylogenetics. J General and Applied Microbiol 51:267-276.

Terry KL, Casey PJ, Beese LS (2006) Conversion of protein farnesyltransferase to a geranylgeranyl transferase. Biochemistry 1545:9746-9755.

Torres-Martínez S, Ruiz-Vázquez RM, Garre V, López-García S, Navarro E, Vila A (2012) Molecular tools for carotenogenesis analysis in the zygomycete Mucor circinelloides. Methods Mol Biol 898:85-107.

Trieu TA, Navarro-Mendoza MI, Pé rez-Arques C, Sanchis M, Capilla J, et al. (2017) RNAi-based functional genomics identifies new virulence determinants in mucormycosis. PLOS Pathog 13:e1006150.

Tudzynski B, K Hölter (1998) Gibberellin biosynthetic pathway in Gibberella fujikuroi: evidence for a gene cluster. Fungal Genet Biol 25:157-170.

Ukibe K, Hashida K, Yoshida N, Takagi H (2009) Metabolic engineering of Saccharomyces cerevisiae for astaxanthin production and oxidative stress tolerance. Appl Environ Microbiol 75:7205-7211. 
Valcarce G, Munoz L, Nusblat A, Nudel C, Florin-Christensen J (2001) The improvement of milk by cultivation with ciliates. J Dairy Sci 2001 84:2136-2143.

Van de Veerdonk FL, Kullberg BJ, Netea MG (2010) Pathogenesis of invasive candidiasis. Curr Opin Crit Care 16:453-464.

van der Linden, JW, Snelders E, Kampinga GA, Rijnders BJ, Mattsson E, DebetsOssenkopp YJ, Kuijper EJ, Van Tiel FH, Melchers WJ, Verweij PE (2011) Clinical implications of azole resistance in Aspergillus fumigatus. Emerg Infect Dis $17: 1846-1854$.

van-Heeswijck R, Roncero MIG (1984) High frequency transformation of Mucor with recombinant plasmid DNA. Carlsberg Res Commun 49:691-702.

Vandeputte P, Ferrari S, Costa AT (2012) Antifungal resistance and new strategies to control fungal infections. Int J Microbiol 2012:713687.

Vastag M, Kasza Z, Ács K, Papp T, Schwab H, Vágvölgyi C (2004) Cloning and sequence analysis of the glyceraldehyde-3-phosphate dehydrogenase gene from the zygomycetes fungus Rhizomucor miehei. Antonie van Leeuwenhoek 86:111-119.

Vaupotic T, Plemenitas A (2007) Osmoadaptation-dependent activity of microsomal HMG-CoA reductase in the extremely halotolerant black yeast Hortaea werneckii is regulated by ubiquitination. FEBS Lett 581:3391-3395.

Vaupotič T, Veranic P, Petrovič U, Gunde-Cimerman N, Plemenitaš A (2008) HMG-CoA reductase is regulated by environmental salinity and its activity is essential for halotolerance in halophilic fungi. Stud Mycol 61:61-66.

Velayos A (2000) Carotenogenesis en Mucor circinelloides. PhD Thesis. Universidad de Salamanca, Salamanca, Spain.

Velayos A, Blasco JL, Alvarez MI, Iturriaga EA, Eslava AP (2000a) Blue-light regulation of the phytoene dehydrogenase (carB) gene expression in Mucor circinelloides. Planta 210:938-946.

Velayos A, Eslava AP, Iturriaga EA (2000b) A bifunctional enzyme with lycopene cyclase and phytoene synthase activities is encoded by the carRP gene of Mucor circinelloides. Eur J Biochem 267:1-12.

Velayos A, Fuentes-Vicente M, Aguilar-Elena R. Eslava AP. Iturriaga EA (2004) A novel fungal prenyl diphosphate synthase in the dimorphic zygomycete Mucor circinelloides. Curr Genet 45:371-377.

Velayos A, Papp T, Aguilar-Elena R, Fuentes-Vicente M, Eslava AP, Iturriga EA, Alvarez MI (2003) Expression of the carG gene, encoding geranylgeranyl 
pyrophosphate synthase, is upregulated by blue light in Mucor circinelloides. Curr Genet 43:112-120.

Vinokur JM, Korman TP, Cao Z, Bowie JU (2014) Evidence of a novel mevalonate pathway in archaea. Biochemistry 53:4161-4168.

Walker LA, Gow NA, Munro CA (2010) Fungal echinocandin resistance. Fungal Genet Biol 47:117-126.

Wang GY, Keasling JD (2002) Amplification of HMG-CoA reductase production enhances carotenoid accumulation in Neurospora crassa. Metab Eng 4:193-201.

Wang L, Lin X (2012) Morphogenesis in fungal pathogenicity: shape, size, and surface. PLoS Pathog 8:e1003027.

Warrilow AG, Melo N, Martel CM, Parker JE, Nes WD, Kelly SL, Kelly DE (2010) Azole Binding Properties of Candida albicans Sterol 14- œ Demethylase (CaCYP51). Antimicrob Agents Chemother 54:4225.

Wawrzyn GT, Bloch SE, Schmidt-Dannert C (2012) Discovery and characterization of terpenoid biosynthetic pathways of fungi. Methods Enzymol 515:83-105.

Weete JD, Abril M, Blackwell M (2010) Phylogenetic distribution of fungal sterols. PLOS ONE 5:e10899.

Weld RJ, Plummer KM, Carpenter MA, Ridgway HJ (2006) Approaches to functional genomics in filamentous fungi. Cell Research 16:31-44.

Werner N, Gómez M, Baeza M, Cifuentes V, Alcaíno J (2016) Functional characterization of thiolase-encoding genes from Xanthophyllomyces dendrorhous and their effects on carotenoid synthesis. BMC Microbiol 16:278.

Whittaker RH (1969) New concepts of kingdoms of organisms. Science 163:150-160.

Wolff AM, Arnau J (2002) Cloning of glyceraldehyde-3-phosphate dehydrogenaseencoding genes in Mucor circinelloides (Syn. racemosus) and use of the gpd1 promoter for recombinant protein production. Fungal Genet Biol 35:21-29.

Yang J, Xian M, Su S, Zhao G, Nie Q, Jiang X, Zheng Y (2012) Enhancing production of bio-isoprene using hybrid MVA pathway and isoprene synthase in E. coli. PLoS ONE 7 e33509.

Yasmin S, Alcazar-Fuoli L, Grundlinger M, Puempel T, Cairns T, Blatzer M, Lopez JF, Grimalt JO, Bignell E, Haas H (2011) Mevalonate governs inter dependency of ergosterol and siderophore biosynthesis in the fungal pathogen Aspergillus fumigatus. Proc Natl Acad Sci USA 109:E497-E504. 
Yancey PH (2005) Organic osmolytes as compatible, metabolic and counteracting cytoprotectants in high osmolarity and other stresses. J Exp Biol 208:2819-2830.

Yelton MM, Hamer JE, Timberlake WE (1984) Transformation of Aspergillus nidulans by using a trpC plasmid. Proc Natl Acad Sci USA 81:1470-1474.

Zhang DH, Jiang LX, Li N, Yu X, Zhao P, Li T, Xu JW (2017) Overexpression of the squalene epoxidase gene alone and in combination with the 3-hydroxy-3methylglutaryl coenzyme A gene increases ganoderic acid production in Ganoderma lingzhi. J Agr Food Chem 65:4683-4690.

Zhang Y, Eusebio Navarro, José T, Cánovas-Márquez, Almagro L, Chen H, Chen YQ, Zhang H, Torres-Martínez S, Chen W, Garre V (2016) A new regulatory mechanism controlling carotenogenesis in the fungus Mucor circinelloides as a target to generate $\beta$-carotene over-producing strains by genetic engineering. Microb Cell Fact 15:99.

Zhang Y, Luan X, Zhang H, Garre V, Song Y, Ratledge C (2017) Improved $\gamma$ linolenic acid production in Mucor circinelloides by homologous overexpressing of delta-12 and delta-6 desaturases. Microb cell Fact 16:113. 


\section{ACKNOWLEDGEMENT}

To complete a project of such magnitude requires a network of support and I am obliged to many people and would like to thank all of them, but to that Almighty first, without whose blessings nothing is possible.

Word thankful is a piece of my deep gratitude towards my supervisor Prof. Dr. Csaba Vágvölgyi, Head of the Department of Microbiology, Faculty of Science and Informatics, University of Szeged (Hungary Europe), for his constant support, excellent guidance and positive outlook in science as well as in life. His broad knowledge, dynamic and friendly nature inspired me to develop scientific temper. He has provided with independence, encouragement and motivation throughout the course of this work. His time, patience and scientific discussion were assets to my research and his opinion goes beyond any measurable value.

I am extremely thankful to Dr. Tamás Papp and Dr. Árpád Csernetics, for his guidance to plan experiment and make them successfully. I always try to be like him constant inspiration, scientific interest, great insight and guidance throughout my research. His patience and willingness to always help me will be remembered with sincere gratitude. I am also thankful to Dr. Ildikó Nyilasi and Dr. Gábor Nagy (Department of Microbiology, Faculty of Science and Informatics, University of Szeged) for their support, advice and suggestions. I thank all my lab members Ms. Eszter J. Tóth, Ms. Tünde Kartali, Ms. Amanda Grace Vaz, Ms. Csilla Szebenyi, and Mr. Olivér Jáger for their help, support and providing a delightful environment during the long hours in the lab.

During my studies on functional characterization of the mevalonate-isoprenoid biosynthesis pathway genes in filamentous fungi, Dr. Árpád Csernetics and Dr. Gábor Nagy deserve my special thank for thier inspiration, moral support, and friendly nature. They helped me in many ways to complete my dissertation. I would also like to thank to all of the departmental colleagues and staffs for their support.

I would like to thank my lovely wife Mrs. Sariks Singh and my dearest colleagues and friends Mr. Tanmoy Chakravarty, Mr. Dhirendra Singh, Mr. Kabi Chandran Singh, and Payal Chakravarty, for their help, support and friendship throughout my studies.

I would like to thank jointly The University Grant Commission New Delhi India, and Stipendium Hungaricum Tempus Public Foundation Hungary, for awarding me the fellowship in form of Doctoral Research Fellowship. 


\section{SUMMARY}

Members of the subdivision Mucoromycotina, order Mucorales (such as Lichtheimia, Mucor, Rhizomucor and Rhizopus species) are saprotrophic fungi, which also have medical, industrial, biotechnological and agricultural importance. Some species may cause post-harvest damages in agriculture; some members are used as producers of extracellular enzymes, organic acids and carotenoids. Several species belonging to this fungal group are also considered to opportunistic pathogens, which can cause fatal systemic infections (so-called zygomycosis or mucormycosis) in immunocompromised patients. Today ergosterol and its biosynthesis is the major target of the antimycotic agents used in clinics to treat infections caused by Mucoromycotina fungi. The therapy of mucormycosis is still limited because of the intrinsic resistance of these fungi to the majority of the currently used antimycotics (such as azoles).

Metabolites synthesized via the mevalonate-isoprenoid pathway (such as sterols, functional groups of proteins and carotenoids) play an important role in signal transduction, morphogenesis, adaptation to environmental changes and protection against free radicals. In the pathway three molecules of acetyl-CoA are condensed by 3-hydroxy3-methylglutaryl-CoA (HMG-CoA) synthase to form HMG-CoA, which is then reduced to mevalonate by HMG-CoA reductase. Next, conversion of mevalonate to isopentenyl pyrophosphate (IPP, which is the binding block of all isoprenoids) is catalyzed by three enzymes, mevalonate kinase, mevalonate-5-phosphate kinase and diphosphomevalonate decarboxylase. In the isoprenoid pathway, formation of dimethylallyl pyrophosphate (DMAPP) is catalyzed by IPP isomerase, followed by condensation of IPP blocks, result in elongation of the prenyl chain. These steps are managed by prenyltransferases, such as farnesyl- (FPP) and geranylgeranyl pyrophosphate (GGPP) synthases, and form the intermediate geranyl pyrophosphate (GPP), FPP and GGPP. FPP and GGPP are the precursors of ergosterol, carotenoids and functional groups of farnesylated and geranylgeranylated proteins.

To date limited information is available about the function and regulation of the mevalonate-isoprenoid biosynthesis pathway genes in Mucoromycotina fungi. Thus, our aim was to characterize six genes of the $M$. circinelloides mevalonate-isoprenoid pathway, encoding the HMG-CoA synthase $(h m g S)$, mevalonate kinase $(m v k)$, diphosphomevalonate decarboxylase $(d m d)$, IPP isomerase (ipi), FPP synthase (isoA) and GGPP synthase (carG). One of our goals was to improve the carotenoid production of the fungus with modification 
of the mevalonate-isoprenoid pathway. Our next aim was to examine the effect of the overexpression and silencing of the genes on the ergosterol content of $M$. circinelloides and it's effect on the susceptibility to different antifungals, which may lead to identify targets for new antifungal therapy. Little is known about the efficiency of gene silencing can be achieved with different plasmid constructs in $M$. circinelloides, thus among our aims was to compare the characteristics of the mutants harbouring different plasmids for gene knockdown as well.

The following specific objectives have been addressed:

1. Investigation of the transcription of selected six genes under different cultivation conditions involved in the mevalonate-isoprenoid biosynthesis in M. circinelloides.

Effect of cultivation conditions, such as temperature, oxygen tension, light sources, medium composition and incubation time on the transcription of the $h m g S, m v k, d m d$, ipi, isoA and $\operatorname{car} G$ genes.

2. Overexpression and silencing of six genes involved in the mevalonate-isoprenoid biosynthesis in $M$. circinelloides and characterization of the transformants

Development of different plasmid constructs for overexpression and silencing of the $h m g S, m v k, d m d$, ipi, isoA and carG genes in $M$. circinelloides. Transformation experiments with the M. circinelloides MS12 strain. Characterization of the transformants in detail, such as analyzes of micromorphology, carotenoid and ergosterol content, antifungal susceptibility and interaction with macrophages. Comparison of the overexpression and gene silencing efficiency achieved with the different plasmid constructs.

\section{Results}

1. Transcription of six genes under different cultivation conditions involved in the mevalonate-isoprenoid biosynthesis in $M$. circinelloides.

Before the present thesis, our research group designed primers to study the transcription of $h m g S, m v k, d m d$, ipi, isoA and carG genes. The M. circinelloides MS12 strain was cultivated under different cultivation conditions, such as on different media composition, different temperature, light conditions, oxygen tension, which was followed by RNA extraction and cDNA synthesis; the relative transcription levels was analyzed with qPCR. The transcription of six mevalonate-isoprenoid pathway genes was compared 
to each other. The fungus was cultivated in liquid or on solid YNB medium for four days. In both cases, the isoprenoid pathway genes (ipi, isoA and carG) showed higher transcription level than mevalonate pathway genes $(h m g S, m v k$ and $d m d)$, moreover we found that $m v k$ showes the lowest, while ipi the highest transcription level. Light was also played key role and reduced the transcription level when the fungus was cultivated in continuous dark, in comparison with cultures cultivated in continuous light. The short light exposure increased significantly the transcription of carG. The isoA showed higher transcipt level, when $M$. circinelloides MS12 was cultivated under warm white (contains more components in the yellow-red wavelength range), than under daylight (contains more components in the blue wavelength range) source; while the $\operatorname{car} G$ showed higher transcription level under daylight condition. Previous study verified that blue light induce the transcription of $\operatorname{car} G$, moreover our findings presume that red color has similar effect to the transcription of isoA. M. circinelloides is a dimorphic fungus, which can grow in yeast-like morphology under anaerobic conditions. All selected six genes showed an increased transcription level upon cultivation under anaerobic conditions in comparison with cultivation under aerobic conditions. Interestingly, anaerobic followed by one hour aerobic growth increased the transcription of $d m d$ significantly and decreased the transcription of $\operatorname{car} G$ compared to cultures under continuous anaerobic condition, which presume the importance of these genes in the biosynthesis of isoprenoids and metabolites playing role in morphological switch. M. circinelloides grows intensively on $25{ }^{\circ} \mathrm{C}$. Growth temperature at $35{ }^{\circ} \mathrm{C}$ increased the transcription of all six genes, moreover similar effect was observed at suboptimal temprerature at most of the genes in comparison with the control conditions $\left(25^{\circ} \mathrm{C}\right)$. Genes showed the highest transcription level at four hours and eight hours postinoculation, except $\operatorname{car} G$, which showed the highest transcription in 96 hours old cultures. The increased glucose concentration generally resulted decrease in most of the gene transcription, while DHA (in previous studies modified and increased the carotenoid production of mutant $M$. circinelloides strains) significantly reduced the transcription of $h m g S$ and $m v k$ and increased that of ipi in comparison with cultivation on glucose. Addition of $\mathrm{NaCl}$ to the media generally did not changed the transcription of the genes significantly, at the same time fluvastatin significantly increased the transcription of all six genes in comparison with the control. 


\section{Construction of plasmids for overexpression and silencing of $M$. circinelloides six}

genes involved in the mevalonate-isoprenoid biosynthesis and transformation experiments.

For overexpression and silencing of the $h m g S, m v k, d m d$, ipi, isoA and $\operatorname{car} G$ genes five plasmid were constructed for all six genes. For overexpression the genes were amplified with their own promoter and terminator regions or were placed under the control of the $M$. circinelloides glyceraldehyde-3-phosphate dehydrogenase ( $g p d l$ ) promoter and terminator (own and gpd plasmid constructs, respectively). For gene silencing three different plasmid were constructed for all six genes: (1) the genes were inserted between the Mucor gpdl and zrtl promoter regions (pMAT constructs); (2) a fragment of a gene was inserted between the Mucor gpdl promoter and terminator in inverted orientation (as constructs); (3) a fragment of a gene and its reverse complement together with an intron were placed under the regulation of the $g p d l$ promoter and terminator ( $h p R N A$ constructs). The Mucor pyrG and leuA genes were used as selection markers, which complement the uracil and the leucine auxotrophy, respectively. Successful transformation of $M$. circinelloides MS12 strain was carried out with the plasmids; the mutants were selected based on the auxotrophy complementation.

The presence of the plasmids in the mutants was verified with PCR. The plasmid copy number was investigated in the mutants and found as to be $0.3-10$ copy/genom; at the same time fluctuation in the copy number was observed. The relative transcription levels of the overexpressed and silenced genes were also analyzed. We observed an increase in the relative transcription level of the overexpressed genes, which was more prominent in those transformants, harbouring the genes in extra copies under the control of the gpdl promoter and terminator, while gene silencing was most effective when the $h p R N A$ and as constructs were used. Significant decrease was found in the colony forming unit in all transformants, including those which harbour the plasmids with the $p y r G$ and $l e u A$ genes, in comparison with $M$. circinelloides MS12, which can be due to mitotic instability of the plasmids. This was more prominent in transformant harbouring the hpRNA plasmids. In case of several transformants (mainly in four days or older colonies) increased number of hyphal branching and cytoplasmic effusion was observed.

Overexpression of all six genes (primary the $m v k$, ipi and $c a r G$ ) increased the carotenoid content, while silencing of mevalonate pathway genes decreased that in comparison with the wild type. Similarly, significant decrease was found in the ergosterol content with silencing of the genes with $h p R N A$ plasmids. Antifungal susceptibility of the 
mutants was also investigated. Primarily modification of the $d m d$ and ipi, responsible for the formation and isomerization of IPP, respectively, resulted significant difference in the susceptibility to azoles and statins in comparison with $M$. circinelloides MS12. Overexpression of the $h m g S$, ipi and $\operatorname{car} G$ and silencing of the $m v k$ and isoA resulted decrease in phagocytic indexes, while overexpression of the $m v k$ and $d m d$ increased phagocytic indexes. 


\section{1. ÖSSZEFOGALÁS}

A Mucormycotina altörzs Mucorales rendjébe tartozó gombák (pl. Lichtheimia, Mucor, Rhizomucor és Rhizopus fajok) elsősorban szaprotrófok, amik ipari, mezőgazdasági és klinikai szempontból is nagy jelentőséggel bírnak. A rend egyes tagjai raktári kártevők, mezőgazdasági termények károsítói lehetnek; másokat extracelluláris enzimek, szerves savak és karotinoidok előállítására használnak az iparban. A csoportba tartozó számos faj ismert, mint opportunista humánpatogén gomba, amik halálos kimenetelü gombafertőzések (ún. zigomikózisok vagy mukormikózisok) kórokozói lehetnek immunszuppresszált betegekben. A klinikumban a mukormikózisok kezelésére alkalmazott antifungális szerek leggyakoribb támadáspontja az ergoszterin és annak bioszintézise, ugyanakkor a mukormikózist okozó gombák többsége rezisztens a legtöbb ma alkalmazott antifungális szerre (így pl. azolokra).

A mevalonsav-izoprén bioszintézis útvonalon képződő metabolitok (így pl. szterinek, karotinoidok, egyes fehérjék funkciós csoportjai) fontos szerepet játszanak a jelátviteli folyamatokban, a morfogenezisben, a megváltozott környezeti feltételekhez történő alkalmazkodásban és a szabadgyökök elleni védelemben. Az útvonal kezdeti szakaszában a HMG-KoA szintáz általt katalizált reakció során három acetil-koenzim A kondenzációjával jön létre a 3-hidroxi-3-metilglutaril-koenzim A (HMG-KoA), ami a HMG-KoA reduktáz hatására mevalonsavvá alakul. Ezt követően a mevalonsav a mevalonsav kináz, foszfomevalonsav kináz és a difoszfoszfomevalonsav dekarboxiláz által katalizált reakcióban izopentenil pirofászfáttá (IPP) alakul, azon öt szénatomos egységgé, ami minden izoprénvázas vegyület alap építőmolekulája. A bioszintézis izoprén szakaszában egy izomerizációs lépés során jön létre a dimetilallil pirofoszfát (ezt a lépést az IPP izomeráz katalizálja), majd a poliprenil transzferázok katalizálta reakcióban IPP egységek beépítésével hosszabbodik a lánc. Ennek során a farnezil pirofoszfát (FPP) és geranilgeranil pirofoszfát (GGPP) szintáz enzimek katalízise révén jön létre a geranil pirofoszfát, farnezil pirofoszfát (FPP) és geranilgeranil pirofoszfát; utóbbiak gombákban a prekurzorai az ergoszterin, karotinoidok, farnezilált és geranilgeranilált fehérjék funkciós csoportjai képződésének.

Kevés ismerettel rendelkezünk a Mucoromycotina gombák mevalonsav-izoprén bioszintézisében szerepet játszó gének szabályozásáról és az egyes metabolitok bioszintézisében betöltött szerepéről. Ezért célul tűztük ki, hogy jellemezzük a $M$. circinelloides ezen bioszintézis útvonalában szerepet játszó géneket, köztük a HMG-KoA 
szintázt $(h m g S)$, mevalonsav kinázt $(m v k)$, difoszfomevalonsav dekarboxilázt $(d m d)$, IPP

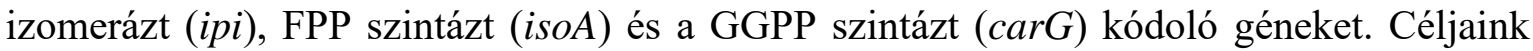
között szerepelt továbbá, hogy a bioszintézis útvonal módosításával karotinoid túltermelő mutáns törzseket hozzunk létre, valamint megvizsgáljuk a gének túlmüködtetésének és csendesítésének hatását a gomba ergoszterin tartalmára és antifungális szerekkel szemben mutatott érzékenységére, ami a későbbiekben hozzájárulhat új antifungális terápiák kifejlesztéséhez. Célul tüztük ki továbbá azt is, hogy megvizsgáljuk a különböző plazmidkonstrukciókkal megvalósítható géncsendesítés hatékonyságát $M$. circinelloidesben.

A munkánk során a következő konkrét célokat fogalmaztuk meg:

1. A M. circinelloides mevalonsav-izoprén bioszintézisében szerepet játszó gének transzkripciójának vizsgálata különböző tenyésztési körülmények mellet.

Különböző környezeti tényezők, így pl. az inkubációs idő, hőmérséklet, oxigéntenzió, fényforrás, táptalaj összetétel hatása a $h m g S, m v k, d m d$, ipi, isoA és carG gének transzkripciójára.

\section{A M. circinelloides mevalonsav-izoprén bioszintézisében résztvevő hat gén} túlműködtetése és csendesítése, valamint a transzformánsok jellemzése.

Különböző plazmidkonstrukciók létrehozása a $h m g S, m v k, d m d$, ipi, isoA és carG gének túlmüködtetéséhez és csendesítéséhez. Transzformációs kísérletek a $M$. circinelloides MS12 törzzsel. A mutáns törzsek részletes jellemzése, így pl. a mikromorfológia, karotin- és ergoszterin tartalom, antifungális szerekkel szembeni érzékenység, valamint makrofágokkal történő interakció vizsgálata. A különböző plazmidkonstrukciókkal elérhető túlmüködtetés és géncsendesítés hatékonyságának összehasonlítása.

\section{Eredmények}

1. A M. circinelloides mevalonsav-izoprén bioszintézisében szerepet játszó gének transzkripciójának vizsgálata különböző tenyésztési körülmények mellet.

Jelen doktori munkát már megelőzően a tanszéki kutatócsoportunk primereket tervezett a $M$. circinelloides MS12 törzs $h m g S, m v k, d m d$, ipi, isoA és carG gének transzkripciójának vizsgálatához. Az MS12 törzset különböző körülmények között (így pl. különböző összetételü táptalajon, különböző hőmérsékleten, eltérő fényforrás és oxigéntenzió mellett) tenyésztettük, majd RNS tisztítást és cDNS szintézist követően 
kvantitatív valós-idejű PCR segítségével megvizsgáltuk a gének transzkripciós szintjét. Megállapítottuk, hogy négy nap tenyésztést követően, mind folyadéktenyészetben, mind szilárd táptalajon, a mevalonsav útvonalban résztvevő $h m g S$, $m v k$ és $d m d$ gének alacsonyabb transzkripciós szintet mutatnak, mint az ipi, isoA és carG. Az mvk gén esetében határoztuk meg a legalacsonyabb, míg az ipi esetében a legmagasabb relatív transzkripciós szintet. Megállapítottuk, hogy az állandó megvilágítás pozitívan hat a gének transzkripciójára a sötétben történő neveléshez képest, továbbá, hogy a sötétben történö nevelést követő rövid idejü megvilágítás különösen a carG gén transzkripciójára van hatással. Az alacsonyabb színhőmérsékletü, a sárga-vörös hullámhossz tartományban intenzívebb warm white fényforrás az isoA, míg a magasabb színhőmérsékletü, a kék tartományban intenzívebb daylight fényforrás a $\operatorname{car} G$ gén transzkripciójára volt pozitív hatással. Korábban már igazolták, hogy a carG gén transzkripcióját a kék fény indukálja, ugyanakkor eredményeink szerint a vörös fény az isoA kifejeződésére van hasonló hatással. A M. circinellodies egy dimorf gomba, vagyis bizonyos körülmények között (így pl. anaerob tenyésztés esetén) az amúgy fonalas gomba élesztőszerü növekedésre képes. Anaerob körülmények között mind a hat gén esetében magasabb transzkripciót mértünk, mint aerob körülmények között, továbbá az anaerob körülmények között történő tenyésztést követő, egy órán át tartó aerob nevelés fokozta a $d m d$, valamint csökkentette a carG transzkripcióját, ami feltételezi ezen gének fontos szerepét a morfológiai váltásban szerepet játszó metabolitok bioszintézisében. A gomba legintenzívebben $25^{\circ} \mathrm{C}$-on nö. 35 ${ }^{\circ} \mathrm{C}$-on mindegyik, illetve az optimálistól alacsonyabb hőmérsékleten a legtöbb gén esetében magasabb transzkripciót mértünk, mint $25^{\circ} \mathrm{C}$-on. A tenyésztési idő tekintetében, a $\operatorname{car} G$ gén kivételével, a leoltást követő négy és nyolc óra után mértük a legmagasabb relatív transzkripciós szinteket, míg a carG esetében ez négy nap tenyésztést követően volt megfigyelhető. A glükózkoncentráció fokozása általában csökkentette a gének kifejeződését, míg a különböző szénforrások közül a dihidroxi-aceton (amely korábbi vizsgálatokban fokozta, illetve módosította egyes mutáns $M$. circinelloides törzsek karotinoid termelését) az ipi és isoA gének kifejeződését fokozta, míg a $h m g S$ és $m v k$ génekét csökkentette a glükózhoz képest. Míg a különböző koncentrációban alkalmazott $\mathrm{NaCl}$ általában nem, ugyanakkor a fluvasztatin szignifikánsan növelte a gének transzkripcióját a kontrollhoz viszonyítva. 


\section{Plazmidok létrehozása a $M$. circinelloides mevalonsav-izoprén bioszintézisében}

résztvevő hat gén túlmüködtetéséhez és csendesítéséhez, valamint a gomba transzformációja a plazmidokkal

A $h m g S, m v k, d m d$, ipi, isoA és carG gének túlmüködtetéséhez és csendesítéséhez öt-öt plazmidot hoztunk létre. A gének túlmüködtetéséhez azokat saját szabályozó régióikkal szaporítottuk fel vagy a $M$. circinelloides glicerinaldehid-3-foszfát dehidrogenáz 1 ( $g p d l)$ promóter és terminális régiók szabályozása alá helyeztük (own és gpd konstrukciók). A gének csendesítéséhez három konstrukciót hoztunk létre: (1) a géneket az egymással szemben elhelyezkedő $M$. circinelloides gpdl és zrtl promóter régiói közé építettük (pMAT konstrukciók); (2) a gének egy szakaszát a gpdl promóter és terminális régiók közé építettük fordított orientációban (as konstrukciók); (3) a gének egy szakaszát, valamint annak reverz komplementerét egy intronnal együtt építettük a gpdl szabályozó régiók közé ( $h p R N A$ konstrukciók). A plazmidok az uracil vagy a leucin auxotrófia komplementálásáért felelős $M$. circinelloides pyrG vagy leuA szelekciós markergéneket hordozták. A plazmidokkal sikeresen transzformáltuk a M. circinelloides MS12 törzsét, a transzformánsokra az auxotrófia komplementáció alapján szelektáltunk.

A létrehozott mutánsokat részletesen jellemeztük. Igazoltuk, hogy a bejuttatott plazmidok jelen vannak a gombában. Megállapítottuk, hogy azok relatív kópiaszáma 0,3 és 10 kópia/genom között mozog, ugyanakkor az átoltások során ingadozást találtunk a plazmidok kópiaszámában. Megvizsgáltuk a gének transzkripciójában bekövetkezett változásokat. Megállapítottuk, hogy a gének túlmüködtetése általában akkor hatékonyabb, amikor a gének a gpdl promóter szabályozása alatt állnak, a gének csendesítése pedig a $h p R N A$ és as konstrukciók alkalmazása esetén bizonyult a leghatékonyabbnak. Megállapítottuk, hogy az MS12 törzshöz képest minden transzformáns esetében, így a leucin és uracil auxotrófia komplementálásáért felelős géneket tartalmazó plazmidokat hordozó transzformánsok esetében is, szignifikáns csökkenés tapasztalható a spórák telepképző képességében, ami feltehetőleg a plazmidok mitótikus instabilitásából eredhet. A gének hpRNA konstrukciókkal történő csendesítése esetében ez a csökkenés még nagyobb mértékü volt. Számos transzformáns esetében, főként idősebb tenyészeteknél, a hifa elágazások és citoplazma kiáramlások számának növekedését tapasztaltuk.

A gének túlmüködtetése (különösen az $m v k$, ipi és carG gének) a karotintermelés fokozódását, továbbá a mevalonsav gének csendesítése annak csökkenését eredményezte, valamint csökkenést tapasztaltunk az ergoszterin tartalomban a gének hpRNA konstrukciókkal történő csendesítése során. Megvizsgáltuk a mutánsok antifungális 
szerekkel szembeni érzékenységét is. Megállapítottuk, hogy különösen az izoprén út kezdeti szakaszában résztvevő, az IPP szintéziséért és izomerizációjáért felelős $d m d$ és ipi gének módosítása eredményezett jelentős változást a gomba azolokkal és sztatinokkal mutatott érzékenységében. Megvizsgáltuk továbbá a mutánsok MH-S makrofágokkal történő interakcióját, és megállapítottuk, hogy a $h m g S$, ipi és carG gének túlmüködtetése, valamint az $m v k$ és $i s o A$ csendesítése a fagocitózis index csökkenését, míg az $m v k$ és $d m d$ túlmüködtetése annak növekedését eredményezte. 


\section{SUPPLEMENTARY MATERIALS}

Suppl 1. Plasmids used in this study for construction of vectors.

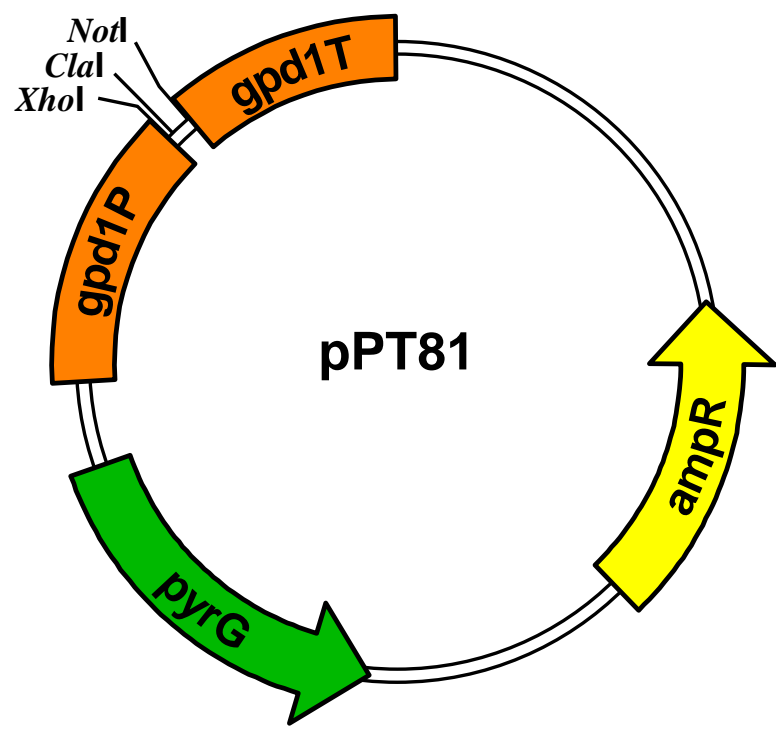

Plasmid pPT81 harbours the Mucor gpdl promoter and terminator (gpdlP and $g p d 1 \mathrm{~T})$, the ampicillin resistance gene $(a m p R)$ and $p y r G$, responsible for the complementation of the uracil auxotrophy.

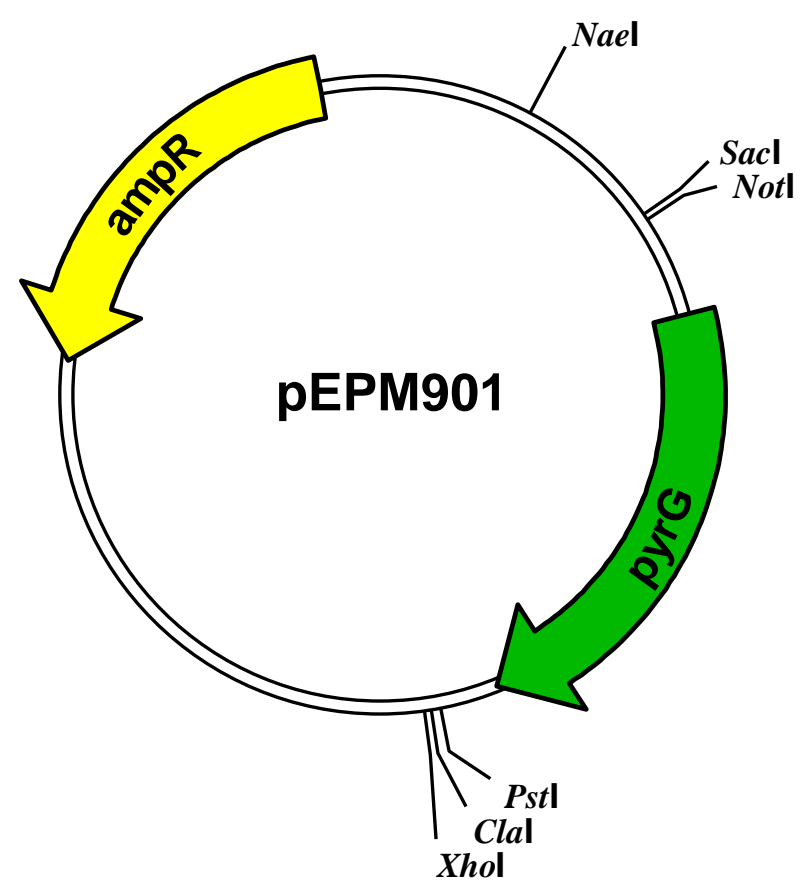

Plasmid pEPM901 harbours the Mucor pyrG gene, responsible for the complementation of the uracil auxotrophy and ampicillin resistance gene $(a m p R)$. 


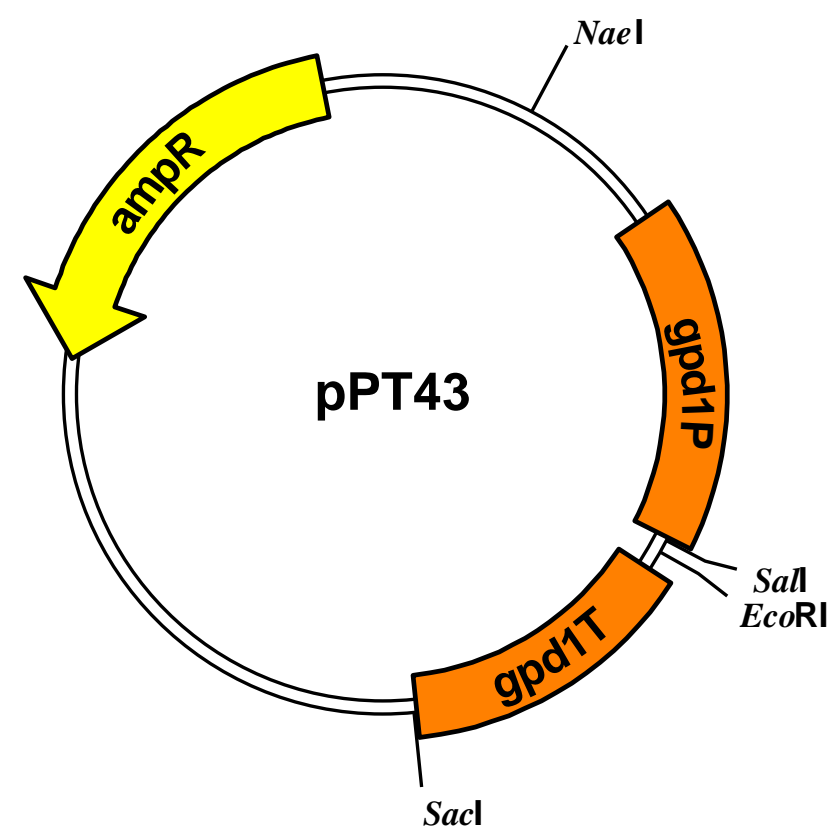

Plasmid pPT43 harbours the Mucor gpdl promoter and terminator (gpdlP and $g p d 1 \mathrm{~T})$ and the ampicillin resistance gene $(a m p R)$.

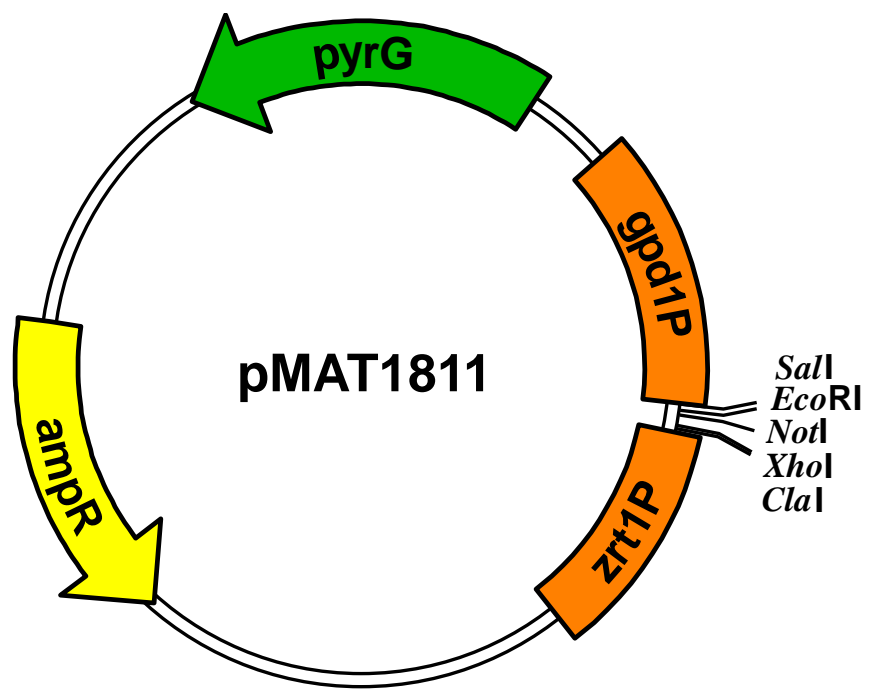

Plasmid pMAT1811 harbours the Mucor gpdl promoter (gpdlP) and zrtl promoter ( $z r t 1 \mathrm{P})$ facing to each other, the Mucor pyrG gene, responsible for the complementation of the uracil auxotrophy and ampicillin resistance gene $(a m p R)$. 
Suppl 2. Nucleotide sequences of $M$. circinelloides genes used in this study and these sequences were collected from the $M$. circinelloides genome database (https://genome.jgi.doe.gov/Mucci2/Mucci2.home.html).

Grey - upstream and downstream from the coding region, red - coding region, blue untranslated region, black - intron.

\section{hmgS}

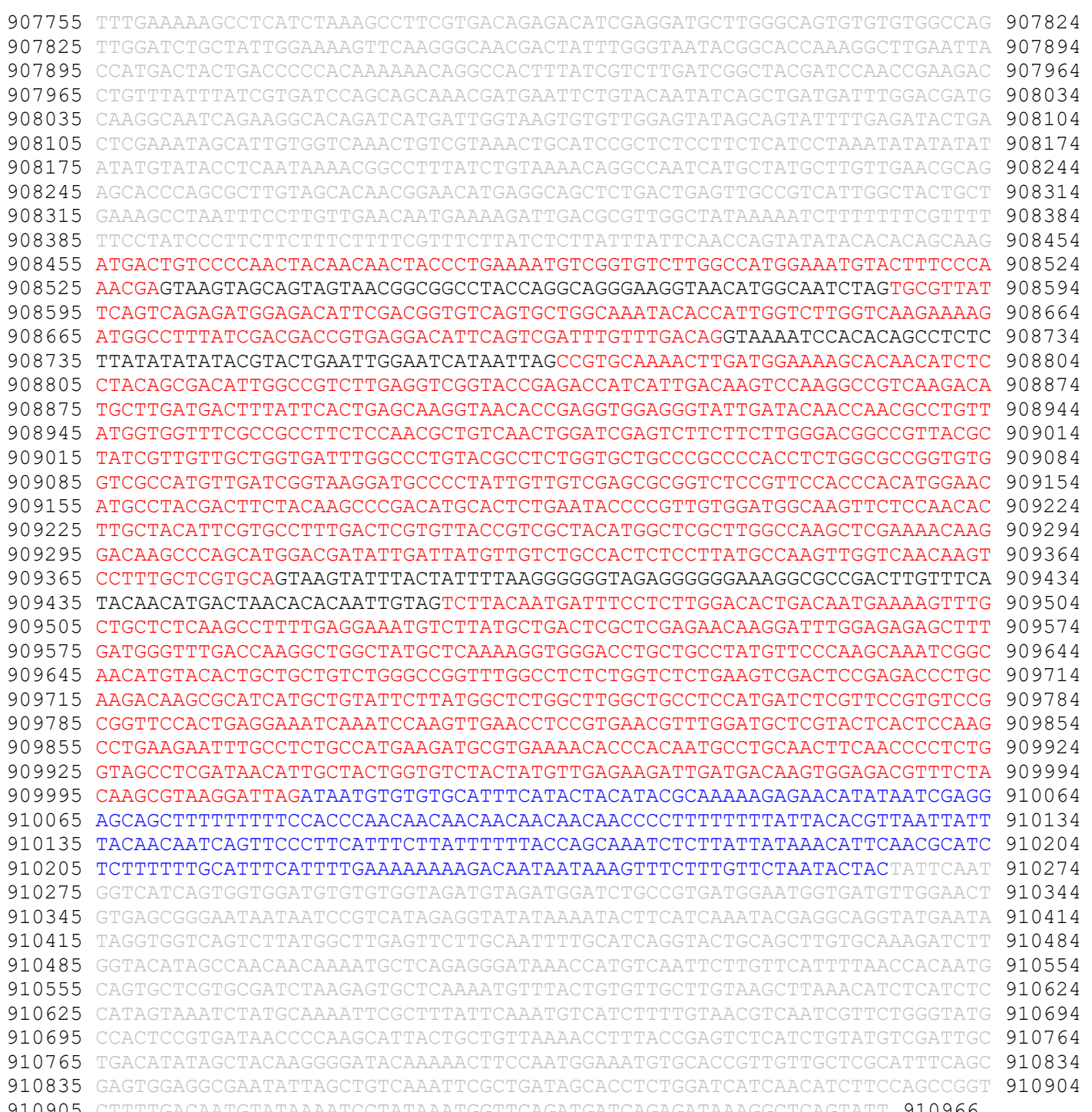




\section{$m v k$}

2391782 TTATGTATAAACAAGAGATAAAATCAAAAGATTTAGGCAATGGTGACCTCAACCTCAACTCCGGGTTCGA 2391713

2391712 TGGAGATGGAGGTGATTGCTTAACGATCTCAGAGGGAGAGTGCAAGTCGATCAAACGCTTGTGGATTCT 2391643

2391642 CATCTCGAAACGATCGAAGGTCTCGGAACCGTTACCACAAGGAGACTTACGGGTGGTGATACGGAGAACC 2391573

2391572 TTGGTGGGGAGACGAACGGGACCCTTGACCTTAAGTTGCTTGTCCTTGGCACGTTGGATGAGGTCAGAGG 2391503

2391502 AGACCTTCTCGAGGTTCTTGACGTTGCGAGAAGTCAAGGTGATACGGATACGATGGATCCTATAAGACAG 2391433

2391432 GGTCAACATTGCAGTTCCAACATTTGGATGTCTCCCGATTGTGTCATAACTTACTTGGCCTCAACTTGTT 2391363

2391362 CCTCCAATCCGGGCTTGGCAGCAGACTTAGCCTACATGTGAAGAATATCATAAATAACCATCGCCTCTCT 2391293

2391292 TCCTATCCATTGAATACATACAGCAATAGCAGACATTTTTAGTGTAAATGAAAAAGAAAGAGAAATCAA 2391223

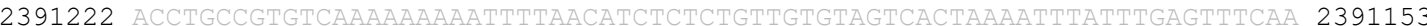

2391152 TGGTGATATATCTTGTCAAACTTACCTGTAACATTGGAACGCTTCTTCTCCTTGAAATGCAATATCGAT 2391083

2391082 ATGACTGCAACTGAACAAAACGAACGCATACTTGTGTCTGCTCCGGGAAAAGTGATTCTGTTTGGCGAGC 2391013

2391012 ACTCGGTTGTCTATCAAAAGGTACGGGCACATGTCATCTACACGGCCTGCTCATCACTGACTGAAGGTTT 2390943

2390942 GCTTCTAGACAGCAGTAGCTGCATCATTGGGATTGAAATCATACCTCTATCTCGAGAACAGAAAGGATGA 2390873

2390872 TTTGATCAAGCTCGTTCTGCCTGACGTTGGCATCGAGAGAAACTGGAAGCTCAGCGACATTCCATTGCGA 2390803

2390802 TTTGAATACCCTGATTCAGGTACCATTTGATCTACAAACCTAAATATCGTGTCTGGTGCAAAATCATCCT 2390733

2390732 TACATTTCATTGTGTAATATATTAGATACCCGTCATCCTGATGATATGCCCGCTGAACTCAAGGCTCGTC 2390663

2390662 TTGTCGAATTATCTGGACCCACTACCAAAGACTCTCAGATCCAGGCACTCATGGCCTTTTTATACCTCTT 2390593

2390592 GATCATCATGGAATCCAAAGCACAAGAGCCATTGACACATGGTCTGACTGTTTGTGTGCGCTCTTTCCTC 2390523

2390522 CCTGTGAGCGCTGGATTGGGTAGTTCTGCCAGTTATTCCGTTGCCATTGCAACCGCCTTATTGCTTCTCA 2390453

2390452 ACAACAAGATTCCCGTTGATTTCAACTCGAGACCTGAAAGAGAGACCTATCTTGAAAAAGTGAACGCTTA 2390383

2390382 TGCATTCAAGGCAGAAGAAGTGATTCATGGTAATCCTAGCGGTGTCGATAATGCGGTAGCCACATACGGT 2390313

2390312 GGAGCAAAGACATTCATGCGCGGACAAGGATTTGCTACATTGGAAGGCTTTAAATCATTGCGTTTGCTGC 2390243

2390242 TCACAAACACCAAGGTTCCCAGATCTACCAGCGCATTGGTAGCTGGTGTAGGCGAGAAGCGTAAAAAGTA 2390173

2390172 TCCTGAGGTTGTGGAGCCCATGCTTGATTCAATGCACGGGATCGCTATTCGTTGTCGTGATGCTTTCAAG 2390103

2390102 AAATTACAAGATAATGAATTGACTACCGAGGCACTCATGGACGAGATTGAAGATTTGGTTACATTAAATC 2390033

2390032 ACTGTTTGCTCGATGGACTTGGTGTGAGCCATCCAAGCTTGGAGAAAGTCAGATCTATCACTGCTCAATC 2389963

2389962 AGGTCTCAAAACCAAATTAACGGGTGCTGGAGGCGGTGGATGCGCTGTTACCTTTATTCGTGATGATGTT 2389893

2389892 CCTCAAGAGATGATCGATCAGGTCATGCTCAATTTACAGTCAGAGGGATTCGATTGCTATCAAACATCTG 2389823

2389822 TTGGTGGTACTGGTGCTGATGCTGTCATCCTTAGTAAGGATGAAACAGATGAATGGCTTCTCGAATCTAA 2389753

2389752 TCGTGATACATTGGAACAATACTTTCAAAACTAAAACTACATTTAATTAAACTTAATTCTACTAATTTAT 2389683

2389682 ACAGAAAACTTACTTTTAACTGTCTACTATTCACGAGAAACCCTTTTAAAATGGTTGATCATTTTGGATT 2389613

2389612 TGGCTCTATCAGGATGCCCCACGTTTGTATCAACAGGAACAACTCATACAAGGGAAGACAGTATACTTGC 2389543

2389542 AATGCTCCTTACTACCCAAAAGAACGCAAAACACAGCGAAATTTGTGTGCATTTCATGCGTGTTTTCTCA 2389473

2389472 TGTTAAAAGCCGTTTTAGAATGCCAGAGTTGCACCAAACAATTGCTCTCATCCACCAGTAACTGTCTGAC 2389403

2389402 GCCTTTGCTAAACTTTTTTGAGATCATCAAAAATTGGCTCAAAAGCATGAGGTACCCCTCATGATTTATG 2389333

2389332 TCGAAAATCAAACGATCGCGTTTTTGAACGCAAAAATGAAGACTTTTACTAAGGCACACGAGCCTTCCCC 2389263

2389262 ACTTTGGGTGGTTTCTTGACCTACGCTGCGCTTCGGTAGAAACCAACCTACGTGCAGGCGGCTCGCGCTG 2389193

2389192 CCGCGGGCCTMTTGGGGGGTTTATCAAAAGATGCTGTGTAGAGATGTTGCAAATCATGATTAGAAAGTCG 2389123

2389122 GATGCCCACACCTTACAATCACTCTGGCGTGAGCTTTACCCCACTTCGGATGGTTTCTTGACCTACGCTG 2389053

2389052 CGCTTCGGTAGAAACCAACCTACGTGGGGTGGGG 2389019 


\section{dmd}

2683125 GTCGCAATCCAAGTAGCATGTTGGTATGAAAAAAGCAAAAAGAGAAGGTTTTCATGAATTAACCCCGGAA 2683056

2683055 AATGCTTTTCAAAGTTCAACTGACAGCGGATGCTAAACATGCCAGCACTGTGGGTTAGATGGCCTTATGA 2682986

2682985 GACATCTGTCAGAAATGCACTATATATCCACGTAGGTGCACTATGCCGTGCAATGCGAGATTCACGTATT 2682916

2682915 CTAGGCGTACTGGACTGTAAGCATAGCCTTATTCTACGTGGCCATGCAGAGGTTTTTATACACATTCAAG 2682846

2682845 TCAACAGGCTTGCAGAAATTGCAGAGCACATTTATCACTACTATTCAAGAATTGTGCGGATAGTGCAGCG 2682776

2682775 ATAAACAATCAAGCACAAATGAGCAGTAAACCAGACCAACTTTTTCGATATACAAAGCAGGTTTGCATTA 2682706

2682705 GACAATCAGATACCATGAATAGCGGGGTATGCCGCAGAACAAATTTCACAAACATTTGATGCCGTTTCGT 2682636

2682635 ATCAGCATTTCACTGCCAAACGATATTGCGCTGAACAGAGTCTTGAGAAACACCACTGAGATGGCCATAA 2682566

2682565 AACAGTGCTCCATGCCAGCTACTATCTGATCTGTCATTTGGCCATTCAGCTTGTGCTGTTTGAGCTTCCT 2682496

2682495 GGTTCCACGCACTGTGGATGGCAATTTACACAACTATCGTTTGGGCGCCTTCCTAAACCTAAACCATTGT 2682426

2682425 GTACACCCACCAAAAAAGTTGGATMTGGTATCTCCTTCATCGCGCTCAAAAGGGACAACAATCAAATMTT 2682356

2682355 TACCCTTTCTTCTTCTTCTTCCTCTTCTTTACCCAAACTCATCAGACCAAAATGAAGACTGTTACCTGTA 2682286

2682285 CTGCTCCCGTCAATATTGCTGTAAGTTGGCTTGTCTGATCCCTTGACACTTGTTCTGTTACTGAATAGCA 2682216

2682215 CACCAGGTCATCAAATATTGGGGCAAGCGCGATACAGAGCTTATTTTGCCTACCAACAGCTCCTTGTCTG 2682146

2682145 TCACATTATCTCAAGACATTCTCCACAGCAAGACCACCATCTCTGCTGCCAAGGAGTACGATCATGATCG 2682076

2682075 TCTTTGGTTGAACGGTATCGAAGAAGACATCACCAAGAACAAGCGTATGCACAACTGCTTCCGTGAAACC 2682006

2682005 CGTGCTATTCGTCAAGCCATGGAAGCCAAGGCCGAAGCTGAGGGCAAGCCCATCGAGCCTCTCTCCACCT 2681936

2681935 ACCCCGTGCACGTTTGCTCCGAGAACAACTTCCCCACTGCTGCTGGCCTCGCTTCTTCAGCCTCTGGATT 2681866

2681865 AGCTGCCCTGGTATACACCTTGTCCCAACTGTTTGAATTGACCATCTCCACGTCTGAAGTGTCCAGAATC 2681796

2681795 GCTCGTCAAGGCTCTGGATCTGCCTGTCGCTCTTTGTTTGGTGGGTTTGTTGCTTGGGAGATGGGAGAGA 2681726

2681725 AGGAGGATGGATCTGACTCATATGCTGTGCAAATTGCTCCTGAGACACACTGGCCTGATTTGCAAGCCTT 2681656

2681655 GATCTGTGTGGTGTCTGACGCCAAGAAGGGCACTTCTTCCACCGCAGGCATGCAATCAACCATCAAGACC 2681586

2681585 AGTCTGTTGATGAATGAGCGTATCAACAACATTGTTCCTGCCCGTATGGAAGGTATGAAGAAGGCTGTTT 2681516

2681515 TGGACAAGGATTTCCAGTCATTTGCCGAGTTGACCATGCGTGATTCCAACCAGTTCCACGCTGTCTGCCT 2681446

2681445 CGACACCTACCCTCCCATCTTTTACTTGAACGATACCTCTCGTGCCATCATTCAGCTTATCCACGAATAC 2681376

2681375 AATGCTACTTCGCCCGACGGTAAATTGAAGGCTGCTTATACCTACGACGCTGGACCAAATGCTGTCATCT 2681306

2681305 ATGCCCCCAAGGAGAACATGGCTGAAATCATCCAATTGATCGGCCACTACTTCCCTAGCAACAAGGAGCC 2681236

2681235 CGCTGAATTCTTTGCCAACCCTTACAATGCCATCAGTGGCAAATTGGGCGAGGACTTCCTCCCCAAGGAC 2681166

2681165 CATGCCAAGTTTAACCAAAATGTCATTCCTGTCCAACCCGTTGGCAGTGTCAGCAGATTATTACACACCA 2681096

2681095 AGGTGGATGATGGACCTCGTGTATTAAGCCAAGATGAGTCTCTCTTGAAGGAAGATGGTCTTCCCAAGCG 2681026

2681025 TCTTGCATAGACATCACTGTTATTATCCTTAATCTCTTATTTTACGCATTCTAATCATTAAAACTGCTTT 2680956

2680955 CTGCCCTTTCACAAATAATAATATCATTGTTACACATCGCCATCGTTTCCATGTGCTGATGATTTGGAAT 2680886

2680885 CCCTCAGCAGTGACCGTCAAGAACGCGAAAGAACACACACACATCACCGCAGTTCCATCCATAAAAGAGC 2680816

2680815 AACAGCTTGCGAATATGCAAAATTTCGTGTCTTCTTATTCTCCAATATCTTACAATGACCCCCTCAATTG 2680746

2680745 CCGAATTCGAAGCTAAAGGCTGGTTCTTGTCTCAAGAAGGCATTGACCTTATTGCCGCAGAAAACGATGG 2680676

2680675 TGTGTCCACATTGGAAGACTATATCGCATGTGCTAAAGACGTAAGCCTGAGTATACTACAACCTACACAA 2680606

2680605 CAAGCGCCGCTGATTACTAACACCACGCATGCTCTTCTAGATGGATCTTCGACTCTTGACTACAAAAGGA 2680536

2680535 TTCAACAАAACTGCAGAGAAACCTAGCGAAATACCTAGTCCACTCGTGCTGCAAGTGCTGGAAGTTCGCA 2680466

2680465 ATGTTGCCATGCCATCTGTCAACCAAGTCGAGCACCCGAGATTACTGAGTGTTACCTTCACTGACGGTAG 2680396

2680395 CAAGAAAAAGTACAAAGGCGTAGAAGTACTTGGAAAAGTAGATTGCCTCAAGTAAGTTGATTTCTTGTTT 2680326

2680325 TATCACATTGACCTCACTACTAAACCCCCACTTGTCTGTTACAGGCTTCACACTCCTCCTGGCACAAAAT 2680256

2680255 TTCTGGTTAAAAAACCAATTGAAATCAGGGATCAGATTCTTGTCTTGGGTCCTGATATGCTGACAGAGAT 2680186

2680185 TGGTGGCCATGTGCAAGAGCTGGTGCAAGCTTGGAGGGCTGGAAAGGTAGTTATGATGCAACAACAGGCC 2680116

2680115 ATGTACTGTTACTAACGTCATTTTAAAGCAATTCTTGAAGCGATATCGAGGAAAGTCAGCTGCCAAAGAA 2680046

2680045 GGCGATGATGAATCAGAGCAAGGCCCGCCAGCATTTGTGCCATTTAAAGTCAAGGTAACTACAACTGATT 2679976

2679975 GACTGGATTAGCCTTGATCACTGACGAAGCTTCATGATACAGCCACACACTGTCGATAAAGAGCCAAAGG 2679906

2679905 CCAAGAAACCGGTAGCTTCCAAAGACATTGCTTTGACCAAGAATGACAAGCCAGTCGAAAAAAGAGGAAA 2679836

2679835 GAAAGCAAAAGCAGACAGCAAGCAGACCGACAATAGCAAGCTAGATGACAAAGCAGAGCAATCAGGCGGA 2679766

2679765 AAAAGAGAGCACAAAAAGGAGACTTCTCGAGAGAAAATCAACCATAAGAAGGATGCTGCAAAGGAGAGAT 2679696

2679695 CTGAGCAACCCAAAGACGTCAACAGCAAAGCGAAGAAGGAGGATTCCAAGTCGAATATGGAAGCTAAAAA 2679626

2679625 GGACAAGCGAAAGGGCAAGCCCGAGAAATCAGACGAGTCCATGAACAGAGTCGATAAAAAGGACATTCCT 2679556

2679555 CCCGAGAGAATACCCTTAGAAAACGCTTCCAACAAGGCAGAGAAGTCTGAAAGACGTGAAAGATCTAGAA 2679486

2679485 AGAAGCAAGAAAAGTCTGTGGAACAAAAAGACGATAGTTTGCCAGCTGCAAAGACGAGATCTCAATCCAA 2679416

2679415 ACAAAGCAGAGACAGCCCAGCAGCAGCAGCAGCAGCAACAGAATCTGGAACATCAAGCCCTGCTCCTCAG 2679346

2679345 TCAGGCACAGCGACTCCTACTTCAGCAGCGGACGACAAGAAATCCAGCCGAAAGAAGAAATCTCCCAAGG 2679276

2679275 CACCTCAGCAGGAACAAATCGACAAACACACATCATGGGCAGATGACATTGTCGAACCCACCACACCTGT 2679206

2679205 CAAGCCAAAAGAGAAGCCTGCTAGAAAAAAAAAGTCGACCGCTAGCTCAACAAAGGCTGATACCACTGCT 2679136

2679135 GAATCGAAATTAGAAGAGGATACCACAACATCCACAGTAACCGCTGCAGCACCTGAAAAGAAGAAGAAGA 2679066

2679065 AGCCTAGAAAAGCAAACGAGAAGAAGACGCTTTCTGAAGAACCAAATGACTCTGATGCCAGCAAACAATC 2678996

2678995 ACAACAGCAGCGAAAGAAGGCAAAGAGCAAATCAGCGAATGAGAAAACCGTG 2678944 
ipi (NCBI Acc. No.: AM903092)

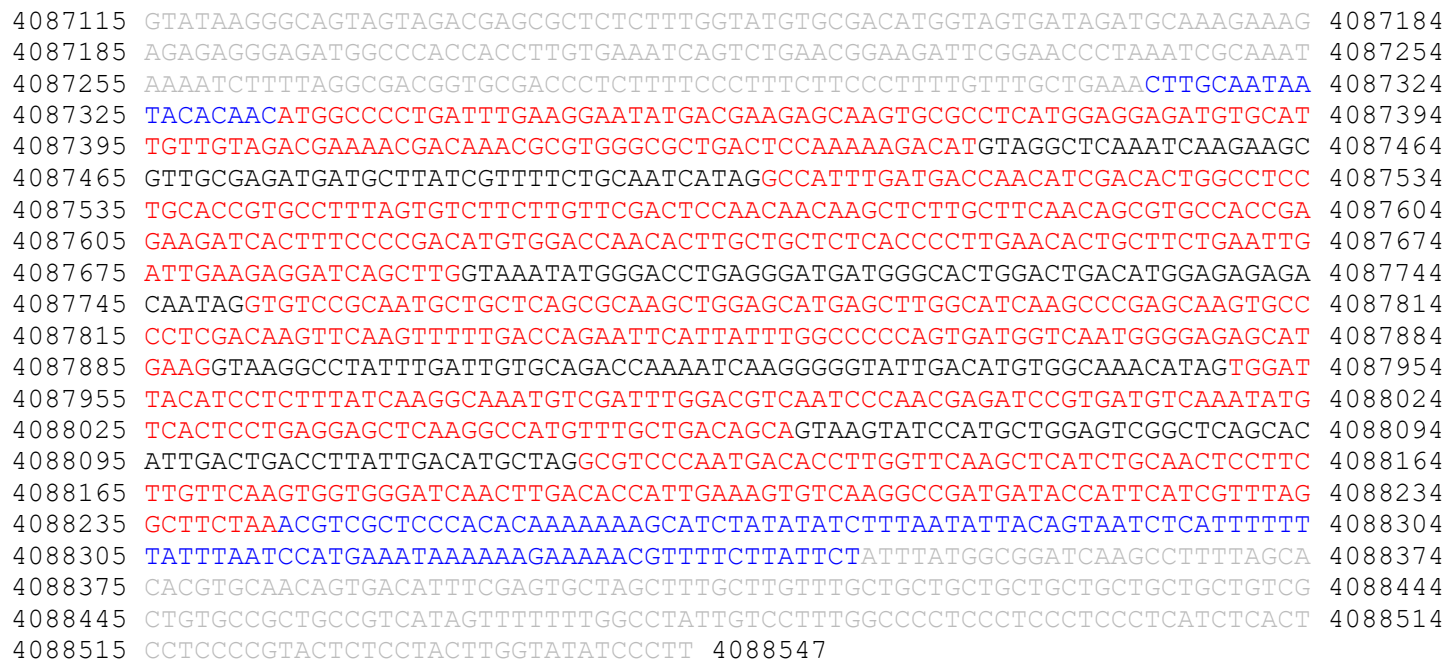

isoA (NCBI Acc. No.: AJ496299)

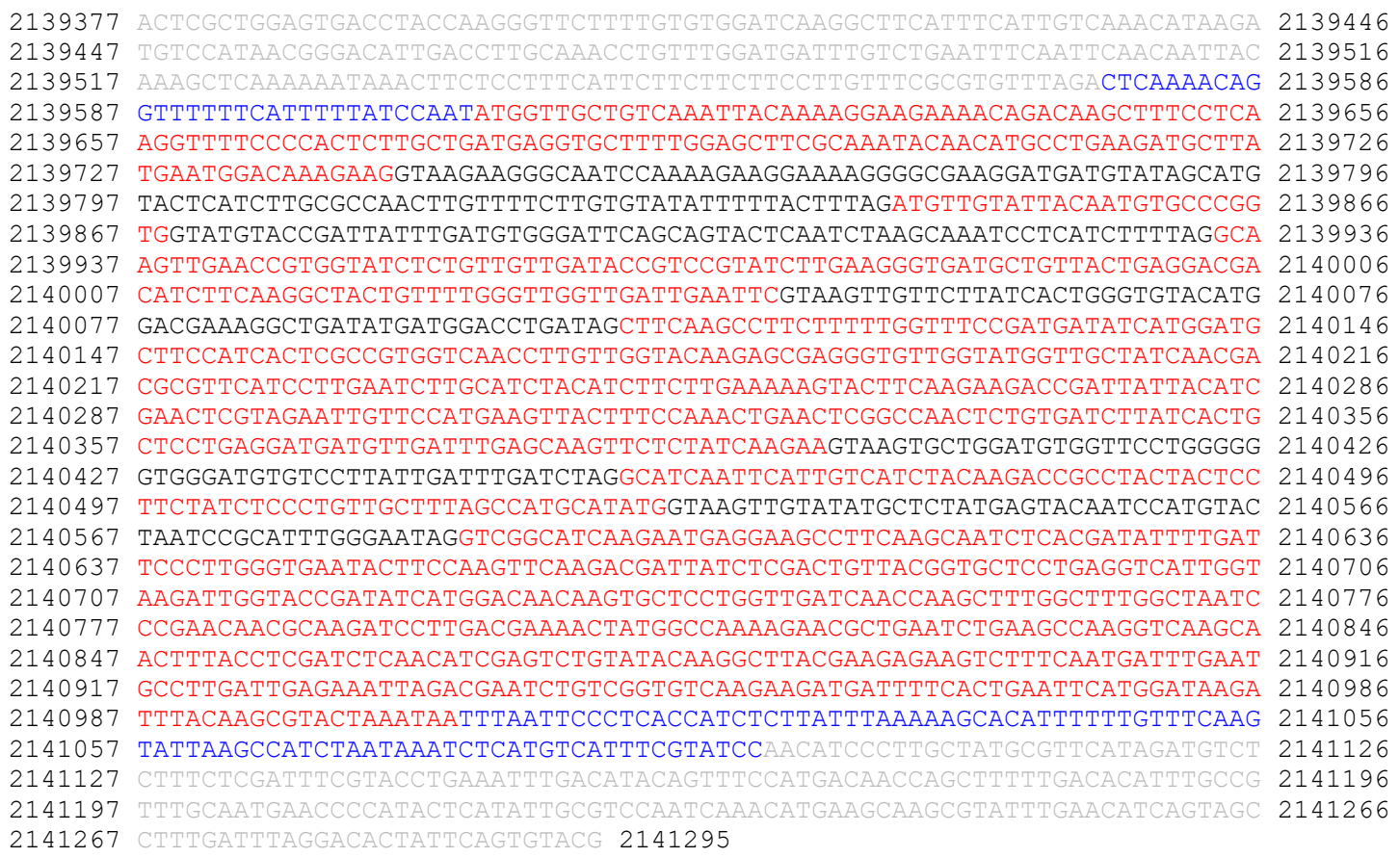


$\operatorname{carG}(\mathrm{NCBI}$ Acc. No.: AJ276129)

4761907 ATTTTTGCATCAAGATGTTTGTGTTTCAAGCAAGATAGATACCTACTGTAATTACAAGCTGAGCATGC 4761976 4761977 CGCAGATCAAATGACAGAAAAAAAGGTGAAACCAGGGCAACAAACCAGCACATGTTGAATTGCCGCCCGA 4762046 4762047 CGCATATATCAAAGCTGGAAAAACTTGGAAACTTTACCTTTTTCTCTCTTCTTTTTCATATTTATTGT 4762116 4762117 CAAATACCATGCTCAACTCACACAACAGAACCGAAGAAAGATCGACCGAAGACGTAAGAGTGTATTTCTC 4762186 4762187 GGAGGGCTAATCTCTGATGACATGCTTGCTAACTGGTAGAAACAGATCATTTTGGAGCCTTACACCTACT 4762256 4762257 TGATATCACAGCCTGGCAAAGATATCCGGGCAAAGTTAATTTCGGCATTCGACCTGTGGCTGCATGTGCC 4762326 4762327 CAAGGACGTGCTGTGCGTAATCAACAAGATTATCGGCATGTTGCATAATGCTAGTTTAATGTAAGTAGTG 4762396 4762397 CTTGTTATCTCGCCATGAGGGGGAGGAAAATGGATACAATTGCTGATATCGATCCCAATACAGGATCGAC 4762466 4762467 GATGTGCAGGATGACTCTGATCTTCGAAGAGGTGTGCCTGTCGCTCACCATATTTATGGTGTACCTCAGA 4762536 4762537 CTATCAACACTGCAAATTATGTCATCTTCTTGGCATTGCAAGAAGTGATGAAGCTGAACATCCCCAGCAT 4762606 4762607 GATGCAAGTGTGCACGGAAGAGCTGATCAATCTGCATCGAGGCCAGGGCATCGAGCTGTACTGGAGAGAC 4762676 4762677 AGCCTGACTTGCCCCACCGAAGAAGAGTACATTGATATGGTCAACAACAGTAAGTGGGCGGTGTAGAGAT 4762746 4762747 CGTGCATTGACTCATGAGTAACACGATCGGGCAGAAACCAGCGGTTTATTACGATTGGCGGTGCGATTAA 4762816 4762817 TGCAAGCAGCAAGTGAAAGTGACATGTGAGTAGATGGTGCCACGTACACACACCTATTCTGGCTGATACT 4762886 4762887 GAACTGTGGGGAGCAGTGATTACACACCGCTCGTCAACATTATAGGCATCCATTTCCAGGTGCGCGATGA 4762956 4762957 CTACATGAACTTGCAATCCACCAGCGTATGTATCAAACTCAAAGGGCGAAGAAGGAGAAGCATGCTTACA 4763026 4763027 TGTGCCTTGATATAGTATACAAACAACAAGGGCTTTTGTGAGGATCTGACAGAGGGCAAGTTTTCATTTC 4763096 4763097 CCATCATTCATGCCATCAGAAAGGACCCTTCCAACCGCCAACTGCTCAACATCATCAGCCAGAAGCCCAC 4763166 4763167 ATCCATTGAAGTCAAAAAGTATGCATTGGAGGTGATTCGCAAGGCAGGCAGTTTTGAATACGTGCGCGAG 4763236 4763237 TTTCTGCGTCAAAAAGAGGCCGAGTCTTTGAAGGAAATCAAGCGTTTGGGTGGTAATCCTTTGCTGGAAA 4763306 4763307 AGTACATTGAGACCATCAGAGTAGAGGCCACCAACGACTAGTAGATTGTCTTTTGGATACCCTGTCAATA 4763376 4763377 AACACACCTTTTCTTTACCCGTCATTTTCGAAATGCTGTACTCGCATGATTGCATTCCATCAACTAAAAC 4763446 4763447 CATAGATGCAATACAAATACCCCCATGATAGCTATAGTATTAGAAACTCTTGTTGAGAGAAAAGGTGATG 4763516 4763517 CAAAGAGTACTACTATCATCCTACTGATAAACCTGACTTGAAGCGTTTACCTTTATAAATAAAGGCACCG 4763586 4763587 TCCTCTTGTCAAG 4763599 